\title{
Integral Equation Methods for Rough Surface Scattering Problems in three Dimensions
}

\author{
Dissertation \\ zur Erlangung des Doktorgrades \\ der Mathematisch-Naturwissenschaftlichen Fakultäten \\ der Georg-August-Universität zu Göttingen
}

vorgelegt von

Eric Heinemeyer

aus Peine

Göttingen 2008 
D7

Referent: $\ldots \ldots \ldots \ldots \ldots \ldots \ldots \ldots \ldots$ Prof. Dr. Roland Potthast Korreferent: $\ldots \ldots \ldots \ldots \ldots \ldots \ldots \ldots \ldots \ldots \ldots \ldots$. Drof. Dr. Rainer Kreß Tag der mündlichen Prüfung: 10ter Januar 2008 


\section{Contents}

Introduction xiii

1 The rough surface scattering problem . . . . . . . . . . xiii

2 BIE for rough surface scattering . . . . . . . . . . . xv

2.1 Non-integrability of fundamental solution . . . . . . . . . . . xvi

2.2 A faster decaying fundamental solution . . . . . . . . . . . xvii

2.3 An equivalent BVP . . . . . . . . . . . . . . xviii

3 General ideas and techniques. . . . . . . . . . . . . xix

3.1 How to prove mapping properties of the operators? . . . . . . xix

3.2 How to show the invertibility of the operator $I+K-i \eta S$ ? . . xx

3.3 How to solve the integral equation numerically? . . . . . . . xxi

4 Thesis outline . . . . . . . . . . . . . . . . xxiv

I Theory 1

1 Tools 3

1.1 Results from functional analysis . . . . . . . . . . . . . . . 3

1.1.1 Some results on bounded linear mappings . . . . . . . . . . 3

1.1.2 Some classical function spaces . . . . . . . . . . . . . . . . 4

1.1.3 Function spaces on the boundary . . . . . . . . . . . . . 10

1.1.4 Integral operators over unbounded domains . . . . . . . . . . 11

1.1.5 Adjoint operators . . . . . . . . . . . . . . . . 14

1.2 Results from differential geometry . . . . . . . . . . . . . . 16

1.3 Results from scattering theory for bounded obstacles . . . . . . . . 16

1.4 Properties of 3D fundamental solution . . . . . . . . . . . . . 18

1.4.1 Expansion for $G \ldots \ldots \ldots \ldots . \ldots \ldots$

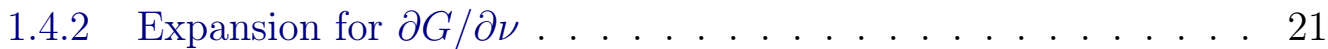

1.5 Results from Fourier analysis . . . . . . . . . . . . . . . 23

1.5.1 Fourier transformation revisited . . . . . . . . . . . 23

1.5.2 Symmetry properties of the Fourier transform . . . . . . . . 26

1.6 Convolution . . . . . . . . . . . . . . . . . . 34

1.6.1 Convolution operators . . . . . . . . . . . . . 35

2 Properties of single- and double-layer potentials 39 
2.1 Boundedness of $S$ and $K \ldots \ldots \ldots \ldots$. . . . . . . . . 40

2.2 Continuity properties of the potential operators . . . . . . . . . . 48

3 Existence and Uniqueness $\quad 51$

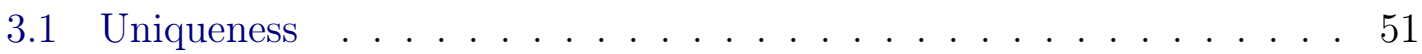

3.2 Invertibility of $I+K-i \eta S \ldots \ldots \ldots \ldots \ldots$

3.2.1 The case of a flat surface . . . . . . . . . . . . . . 54

3.2 .2 The case of a general rough surface . . . . . . . . . . 56

$\begin{array}{lll}\text { II } & \text { Numerics } & 59\end{array}$

4 Tools $\quad 61$

4.1 Notations . . . . . . . . . . . . . . . . . . . . . . 61

4.2 Multi-dimensional Fourier analysis . . . . . . . . . . . . . 64

4.2.1 Discrete Fourier transform . . . . . . . . . . . . . . . 66

4.3 The composite trapezoidal rule . . . . . . . . . . . . . . . . . 69

5 Operator approximations $\quad 75$

5.1 Approximations for weighted differentiable kernels . . . . . . . . . . 76

5.2 Approximations for weakly singular kernels . . . . . . . . . . . . 76

5.2.1 The case of the single-layer potential . . . . . . . . . . 77

5.2.2 The case of the double-layer potential . . . . . . . . . . . 79

5.2.3 The locally corrected quadrature scheme . . . . . . . . . . . 81

6 Nyström methods for rough surface scattering $\quad 87$

6.1 Method I: Discretisation-truncation . . . . . . . . . . . . . . . . . . 89

6.2 Method II: Truncation-discretisation . . . . . . . . . . . . . . . . 90

6.3 The Finite Section Method . . . . . . . . . . . . . . . . . . . . 91

6.4 The Banded Matrix Iterative Algorithm for rough surface scattering . 92

7 The Multi-Section Method $\quad 95$

7.1 Existence and convergence . . . . . . . . . . . . . . . 96

7.2 Applicability to the rough surface scattering problem . . . . . . . . 97

7.3 Numerical realisation . . . . . . . . . . . . . . . . . . . . . . . 99

7.3.1 Linear least squares problems . . . . . . . . . . . . 100

8 Fast matrix-vector mult. for difference kernels $\quad 105$

8.1 The multi-dimensional case for periodic kernel . . . . . . . . . . . . . 107

8.2 The multi-dimensional case for non-periodic kernel . . . . . . . . . 109

9 A new view on canonical grid methods 113

9.1 Canonical grid method revisited . . . . . . . . . . . . . . . . . . 113 
9.1.1 Deriving a kernel decomposition . . . . . . . . . . . . . . . . 114

9.2 A novel approach . . . . . . . . . . . . . . . . . . . 116

9.2.1 On a good choice of Dirac delta sequences . . . . . . . . . 118

9.3 Connections to the classical canonical grid method . . . . . . . . . . 130

9.4 Applying the method to the rough surface scattering problem . . . . 131

9.4.1 Extending the fast method to certain non-difference kernels . . 132

9.5 Alternative kernel functions . . . . . . . . . . . . . . . . . . . 133

9.5.1 Radial basis functions . . . . . . . . . . . . . . . . 133

9.6 Numerical results . . . . . . . . . . . . . . . . . . . . 135

10 Fast method - Implementation details $\quad 139$

$\begin{array}{ll}\text { A Maple Procedures } & 145\end{array}$

$\begin{array}{ll}\text { Bibliography } & 147\end{array}$

$\begin{array}{ll}\text { List of Symbols } & 153\end{array}$ 


\section{Abstract}

We consider the scattering of time harmonic acoustic waves by a sound soft rough surface in three dimensions. The analysis we develop holds in the case that the incident wave is due to a point source situated above the scattering surface. It does however not apply to the case of an incident plane wave.

The first part of the thesis settles the question of existence and uniqueness of the scattering problem. The scattered field is sought as a modified Brakhage-Werner ansatz. This approach yields an boundary integral equation of the second kind in the space of complex valued square integrable functions on the scattering surface. In contrast to the case of bounded obstacles, the integral operators occuring in this equation are not compact so that the Riesz-Fredholm theory is not applicable.

The second part is concerned with the numerical solution of the integral equation. To handle the infinite domain of integration the integral equation is truncated in a first approximation step to an equation on a finite section of the real plane. For this, we introduce a novel truncation scheme called multi-section method for which we can proof convergence. In a second step standard discretisation schemes for integral equations on bounded domains can now be applied.

The discretisation of the truncated equations yields large and dense linear systems that must be solved by iterative methods. It is therefore important to derive fast matrix-vector multiplication schemes. We introduce an alternative derivation of the canonical grid method that uses high order approximations of the Dirac delta distribution. This approach allows a rather simple implementation of the canonical grid method. In addition our algorithm achieves an additional speed. For the case of the single-layer potential operator we show the feasibility of this method by numerical examples. 


\section{Zusammenfassung}

Wir behandeln die Streuung zeitharmonischer akustischer Wellen an einer schallweichen rauhen Oberfläche in drei Dimensionen. Unsere Untersuchungen sind gültig für den Fall, dass die einfallende Welle von einer Punktquelle oberhalb der Streuoberfläche herrührt. Sie sind jedoch nicht auf den Fall einer einfallenden ebenen Welle anwendbar.

Der erste Teil der Arbeit behandelt die Existenz und Eindeutigkeit des Streuproblems. Das gestreute Feld wird in Form eines modifizierten Brakhage-WernerAnsatzes gesucht. Dieses führt zu einer Randintegralgleichung zweiter Art in dem Raum der komplexwertigen quadratintegrierbaren Funktion auf der Streuoberfläche. Im Gegensatz zu dem Fall eines beschränkten Streuobjektes sind die auftretenden Integraloperatoren nicht kompakt, so dass die Riesz-Fredholm-Theorie nicht angewandt werden kann.

Der zweite Teil der Arbeit beschäftigt sich mit der numerischen Lösung der Integralgleichung. Um den unendlichen Integrationsbereich zu handhaben, wird die Integralgleichungen in einem ersten Approximationsschritt auf einen endlichen Bereich der reellen Ebene reduziert. Wir führen hierzu ein neues Abschneideverfahren, genannt multi-section method, ein, für welches wir Konvergenz zeigen können. In einem zweiten Schritt können nun Standard-Diskretisierungsverfahren für Integralgleichungen auf endlichen Gebieten angewandt werden.

Die Diskretisierung der reduzierten Gleichung liefert große dicht besetzte lineare Gleichungssysteme, welche mittels iterativer Methoden gelöst werden müssen. Es ist daher erforderlich, schnelle Matrix-Vektor-Multiplikationsmethoden zu entwickeln.

Wir stellen einen alternativen Zugang zu der canonical grid method vor, welcher auf Approximationen der Dirac'schen Delta-Distribution hoher Ordnung basiert. Diese neue Interpretation erlaubt eine recht einfache Implementierung. Darüber hinaus erreicht unser Algorithmus einen Geschwindigkeitsvorteil. Für den Fall des Einfachschichtpotentialoperators belegen wir die Anwendbarkeit dieser Methode durch numerische Beispiele. 


\section{Acknowledgment}

I thank my advisor Prof. Dr. Roland Potthast for his support during my research. He gave me the freedom to follow some of my own ideas in great depth and in my own manner, arranged for two wonderful research trips to the University of Reading and introduced me to Prof. Simon Chandler-Wilde.

My warmest thanks go to Prof. Dr. Rainer Kreß, who agreed to be the second referee for my thesis despite the enormous number of graduate students finishing together within a couple of month.

The cooperation with Prof. Simon Chandler-Wilde during the last three years was very fruitful, he arranged for me to present some of my work during the BICS conference in Bath and I thank him for all hints and the interest he took for my work. I enjoyed my stays in Reading very much having stimulating discussions with Dr. Marko Lindner and others pointing me to some interesting literature for my work. The use of RBF for the interpolation scheme was suggested by Prof. Schaback, whom I am indebted to for discussions and many useful hints.

I thank the Deutsche Forschungsgemeinschaft represented through the Graduiertenkolleg: "Identifikation in mathematischen Modellen" for the financial support in the last three years and all my fellow PhD-students in the institut. Above all, my deepest thanks go to my wife for being the person she is and my parents for their never ending support. 


\section{Introduction}

\section{The rough surface scattering problem}

This thesis is concerned with boundary integral equation methods for what has been termed rough surface scattering problems in the engineering literature.

A rough surface is a non-local perturbation of an infinite flat plane, such that the whole surface lies within a finite distance of the original plane. We treat the special case, where the surface is given as graph of some bounded continuous function, which we refer to as the surface height function, i.e. for a function $f: \mathbb{R}^{2} \rightarrow \mathbb{R}$ the scattering surface denoted by $\Gamma$ is given through

$$
\Gamma=\left\{(\boldsymbol{x}, f(\boldsymbol{x})) \in \mathbb{R}^{3}: \boldsymbol{x}=\left(x_{1}, x_{2}\right) \in \mathbb{R}^{2}\right\} .
$$

We assume that $f$ is in $B C^{1, \alpha}\left(\mathbb{R}^{2}\right)$, the space of bounded continuously differentiable functions with bounded and uniformly Hölder continuous derivatives, which makes the scattering surface $\Gamma$ a Lyapunov surface. Thus $f$ is bounded and without loss of generality we can assume that there exist constants $f^{+}>f^{-}>0$ such that

$$
f^{-} \leq f(\boldsymbol{x}) \leq f^{+} \quad \text { for all } \quad \boldsymbol{x} \in \mathbb{R}^{2} .
$$

This thesis focuses on one typical problem from the application of outdoor sound propagation, namely the scattering of an incident acoustic field by a sound-soft surface. That is we are interested to compute the propagation of the reflected wave in the medium of propagation occupying the perturbed half-space above the scatterer that we denote by

$$
D:=\left\{\left(\boldsymbol{x}, x_{3}\right) \in \mathbb{R}^{3}: x_{3}>f(\boldsymbol{x}), \boldsymbol{x} \in \mathbb{R}^{2}\right\} .
$$

We note that for later studies on the dependence of the scattered field on the boundary it is convenient to sometimes write $\Gamma_{f}$ for $\Gamma$ and $D_{f}$ for $D$ to make the dependence on the boundary more explicit. In the case of the constant function $f: \mathbb{R}^{2} \rightarrow \mathbb{R}$, $\boldsymbol{x} \mapsto h$, for $h \in \mathbb{R}$, we write $\Gamma^{h}$ and $D^{h}$.

Time-harmonic acoustic waves, that are waves with a time dependence of the form $e^{-i \omega t}$, are modelled by the Helmholtz equation

$$
\triangle u+\kappa^{2} u=0 .
$$




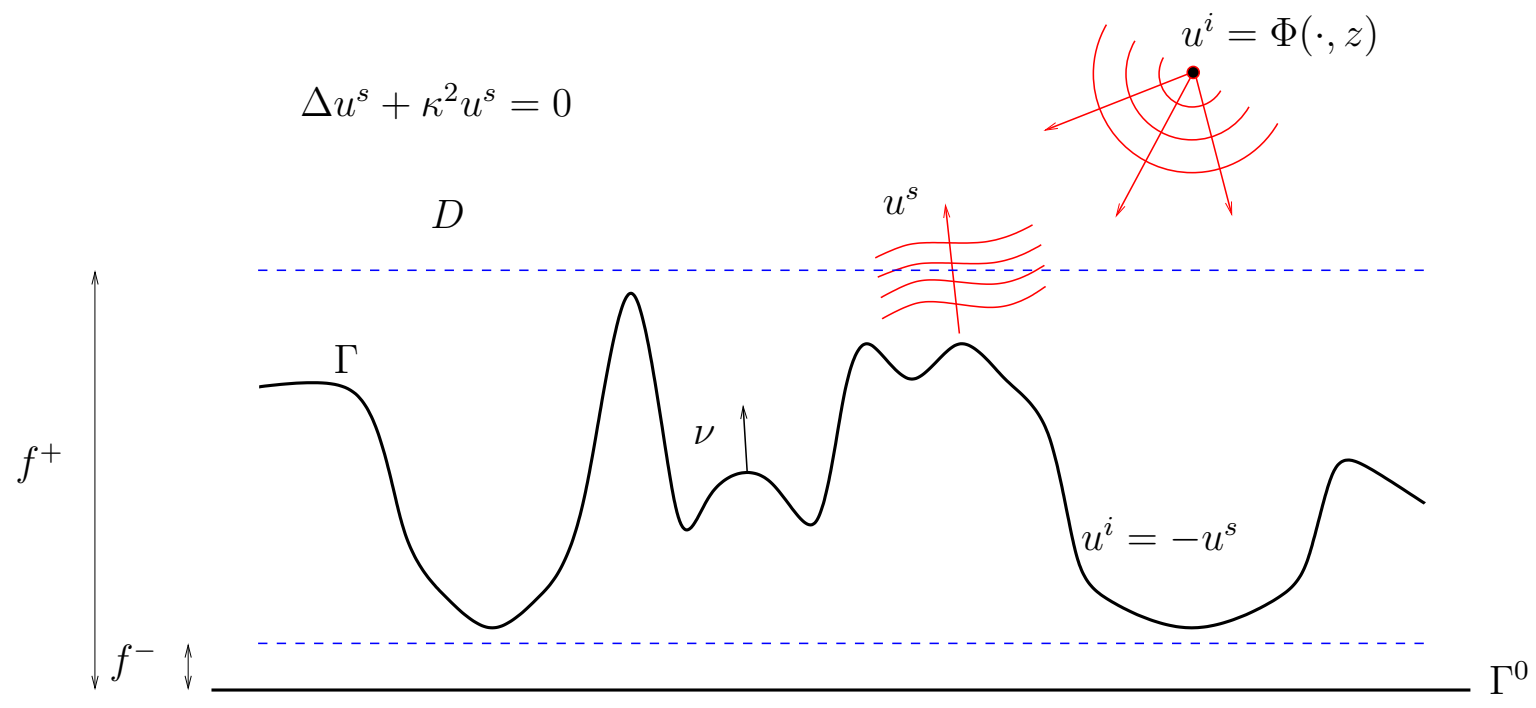

Figure 1: Geometrical setting of the scattering problem.

Here $\kappa=\omega / c_{0}$ stands for the wavenumber, $\omega$ the frequency and $c_{0}$ the speed of sound. Though we are mainly interested in the case of positive real wavenumbers, we consider the more general case of wavenumbers with a positive imaginary part. Thus we have the natural decomposition

$$
\kappa=\kappa_{r}+i \kappa_{i} \in \tilde{\mathbb{C}}
$$

where $\kappa_{r}>0$ denotes the realpart, $\kappa_{i} \geq 0$ the imaginary part of the wavenumber and

$$
\tilde{\mathbb{C}}:=\{z \in \mathbb{C}: \Re(z)>0, \Im(z) \geq 0\} .
$$

Physically speaking the case of complex wavenumbers models lossy media that absorb the sound energy.

A sound-soft surface is modelled through a Dirichlet boundary condition. This means that we require the total field

$$
u:=u^{i}+u^{s},
$$

which is the sum of the incident field $u^{i}$ and the scattered field $u^{s}$, to vanish on the boundary, i.e.

$$
u(x)=0, \quad x \in \Gamma .
$$

The analysis we develop is applicable whenever the incident wave is due to sources of the acoustic field located in some compact set $M \subset D$. Since waves with sources in a bounded set $M \subset \mathbb{R}^{3}$ can be represented as superpositions of point sources located in the same set, we will concentrate on the case when the incident field is that due to a point source located at some point $z \in D$, i.e.

$$
u^{i}=\Phi(\cdot, z),
$$


where

$$
\Phi(x, y):=\frac{1}{4 \pi} \frac{e^{i \kappa|x-y|}}{|x-y|}, \quad x, y \in \mathbb{R}^{3}, \quad x \neq y,
$$

denotes the fundamental solution to the Helmholtz equation in $\mathbb{R}^{3}$.

To ensure uniqueness we need to pose some kind of boundary condition at infinity. This can be either done with a growth or radiation condition. In the present case we need a combination of both types. First of all we require that the scattered field is bounded in $D$, i.e.

$$
\left|u^{s}(x)\right| \leq c, \quad x \in D,
$$

for some constant $c>0$. This condition is enough to ensure uniqueness in the case of a wavenumber with positive imaginary part. In the case of a purely real wavenumber $\kappa=\kappa_{r}$ we also require that $u^{s}$ satisfies the following limiting absorption principle: denoting $u^{s}$ temporarily by $u_{(\kappa)}^{s}$ to indicate its dependence on $\kappa$, we suppose that for all sufficiently small $\epsilon>0$ a solution $u_{(\kappa+i \epsilon)}^{s}$ exists and that

$$
u_{(\kappa+i \epsilon)}^{s}(x) \rightarrow u_{(\kappa)}^{s}(x), \quad \epsilon \rightarrow 0 .
$$

The limiting absorption principle plays the role of a radiation condition for real $\kappa$ to single out the physical solution.

Thus the following is the specific problem that is considered, for an illustration of the geometrical setup see FiguRE 1:

Problem 1 (Point source rough surface scattering problem). Let $u^{i}=\Phi(\cdot, z)$ be the incident field due to a point source at $z \in D$. Then we seek a scattered field $u^{s} \in C^{2}(D) \cap C(\bar{D})$ such that $u^{s}$ is a solution to the Helmholtz equation (4) in $D$, the total field satisfies the sound-soft boundary condition (6), and the bound (7) holds. In the case $\kappa>0$, we also require that the limiting absorption principle (8) holds.

\section{BIE for rough surface scattering}

Boundary integral equation methods have been applied very successfully since the beginning of the 20th century to various problems from mathematical physics, including problems from potential theory or scattering theory for acoustic/electromagnetic waves. In the case of smooth bounded obstacles the theory is very well developed and there are very effective ways to utilise the integral equation for the numerical solution of the problems, see e.g. the books of Kress [35], Colton \& Kress [24],[23], McLean [39] or Atkinson [4].

In the case where the scattering object is an unbounded surface the situation is very different. Although integral equations are widely used, especially in the computation of rough surface scattering problems see e.g. the reviews and monographs 
by Ogilvy [44], Voronovich [55], Saillard \& Sentenac [52], Warnick \& Chew [57], and DeSanto \& Martin [25],[26], the mathematical basis of the method is still poorly developed, especially in the 3D case. In fact, there are a number of severe difficulties in extending the theory of BIE methods from bounded to unbounded scatterers.

\subsection{Non-integrability of fundamental solution}

In the case of a purely real wavenumber $\kappa>0$, the standard fundamental solution $\Phi(x, y)$ of the Helmholtz equation has a rather slow decay at infinity, like $\mid x-$ $\left.y\right|^{-(n-1) / 2}$ in $n$ dimensions. Thus the standard boundary integral operators are not bounded on any of the standard function spaces when the surface is unbounded.

We illustrate this in some more detail and take a look at the ordinary single-layer potential

$$
\int_{\Gamma} \Phi(x, y) \varphi(y) d s(y), \quad x \in \Gamma .
$$

The problem is that, due to the slow decay of the fundamental solution in $\mathbb{R}^{3}$ at infinity, the integral (9) converges only if $\varphi$ decreases sufficiently rapidly at infinity. So one could try to work with $\varphi \in \mathcal{S}(\Gamma)$, the Schwartz space of rapidly decreasing functions. But this does not seem to be desirable due to the fact that this is a very small space with a rather unpleasant topological structure. One could think to work with $L^{p}$ spaces instead, but, as the following calculations show in the case where the surface is a flat plane, this is not possible also. Denote

$$
B\left(r_{1}, r_{2} ; \boldsymbol{x}\right):=\left\{\boldsymbol{y} \in \mathbb{R}^{2}: r_{1}<|\boldsymbol{x}-\boldsymbol{y}|<r_{2}\right\}
$$

for positive numbers $r_{1}<r_{2}$. Then we ask whether there exists $p \geq 1$ such that

$$
\lim _{r_{1} \rightarrow 0} \lim _{r_{2} \rightarrow \infty} \int_{B\left(r_{1}, r_{2} ; \boldsymbol{x}\right)}|\Phi(\boldsymbol{x}, \boldsymbol{y})|^{p} d \boldsymbol{y}
$$

exists. Introducing polar coordinates with centre $\boldsymbol{x}$ we calculate

$$
\begin{aligned}
\int_{B\left(r_{1}, r_{2} ; \boldsymbol{x}\right)}|\Phi(\boldsymbol{x}, \boldsymbol{y})|^{p} d \boldsymbol{y} & =C \int_{B\left(r_{1}, r_{2} ; \boldsymbol{x}\right)} \frac{1}{|\boldsymbol{x}-\boldsymbol{y}|^{p}} d \boldsymbol{y} \\
& =C \int_{r_{1}}^{r_{2}} r^{1-p} d r= \begin{cases}\left(r_{2}-r_{1}\right), & p=1, \\
r_{2}^{2-p}-r_{1}^{2-p}, & 1<p<2, \\
\ln r_{2}-\ln r_{1}, & p=2, \\
r_{2}^{2-p}-r_{1}^{2-p}, & p>2,\end{cases}
\end{aligned}
$$

where $C>0$ denotes some generic constant. From these elementary calculations we see that we have two kinds of singularities that we carefully need to balance against 
each other. The local singularity at zero is weakly singular and therefore integrable for $1 \leq p<2$. For $p \geq 2$ it becomes strongly singular or even hyper singular. The non-integrability of $\Phi(x, \cdot)$ over $\mathbb{R}^{2}$, due to it's slow decay, can be interpreted as a singularity at infinity that is strongly singular for $p \leq 2$ and weakly singular for $p>2$. These calculations show that we better replace the function $\Phi$ by some function that has a faster decay, rather than trying to find an appropriate space.

\subsection{A faster decaying fundamental solution}

In order to get a faster decaying kernel we replace $\Phi(x, y)$, following what has been proposed for the analogous 2D rough surface scattering case [63], by an appropriate half-space Green's function for the Helmholtz equation. Specifically, we will work with the function

$$
G(x, y):=\Phi(x, y)-\Phi\left(x, y^{\prime}\right)
$$

where

$$
y^{\prime}:=\left(y_{1}, y_{2},-y_{3}\right),
$$

is the image of $y$ in the flat plane $\Gamma^{0}=\left\{x \in \mathbb{R}^{3}: x_{3}=0\right\}$. The function $G$ is the Dirichlet Green's function for the half-space $D^{0}=\left\{x \in \mathbb{R}^{3}: x_{3}>0\right\}$. In Section 1.4 we show the bound for $y \in \Gamma$,

$$
|\nabla G(x, y)|,|G(x, y)| \sim C \frac{\left(1+x_{3}\right)\left(1+y_{3}\right)}{|x-y|^{2}}, \quad|y| \rightarrow \infty,
$$

cf. (1.22) and (1.28). This decay is fast enough for the single-layer potential operator, given by

$$
(S \varphi)(x):=2 \int_{\Gamma} G(x, y) \varphi(y) d s(y), \quad x \in \Gamma,
$$

and the double-layer potential operator, given by

$$
(K \varphi)(x):=2 \int_{\Gamma} \frac{\partial G(x, y)}{\partial \nu(y)} \varphi(y) d s(y), \quad x \in \Gamma,
$$

where the unit normal vector $\nu(y)$ is directed into $D$, to be well-defined as improper integrals, for every $x \in \bar{D}$ and $\varphi \in L^{2}(\Gamma)$, in particular in the case $\kappa_{i}=0$.

Because, for $x \in \Gamma$,

$$
\int_{\Gamma \cap B_{R}(x) \backslash B_{1}(x)} \frac{1}{|x-y|^{2}} d s(y) \rightarrow \infty, \quad R \rightarrow \infty,
$$

the decay of $G(x, y)$ as $y \rightarrow \infty$ is not fast enough when $\kappa>0$ for $S$ to be well defined as an operator on the space of bounded continuous functions. Thus integral equation methods for the 3D rough surface scattering problem are essentially different from the $2 \mathrm{D}$ case studied in $[16,17,18,63,3]$ and the analysis developed here can not be applied to the interesting case of a plane wave incident. 


\subsection{An equivalent BVP}

To apply integral equation methods we convert the scattering problem into a boundary value problem. For this we seek the scattered field as the sum of a mirrored point source

$$
\Phi^{\prime}(\cdot, z):=-\Phi\left(\cdot, z^{\prime}\right),
$$

plus some unknown remainder $v$, i.e.

$$
u^{s}=v+\Phi^{\prime}(\cdot, z) .
$$

Using the boundary condition

$$
u^{s}+\Phi(\cdot, z)=0 \quad \text { on } \quad \Gamma=\partial D
$$

we obtain the boundary condition on $v$ that

$$
v(x)=-G(x, z)=: g(x), \quad x \in \Gamma .
$$

Clearly $g \in B C_{\infty}(\Gamma)$, the space of bounded continuous functions vanishing at infinity, and it follows from (11) that $g \in L^{2}(\Gamma)$, so that $g \in L^{2}(\Gamma) \cap B C_{\infty}(\Gamma)$. Further, by the dominated convergence theorem we see that $\left\|g_{\epsilon}-g\right\|_{L^{2}(\Gamma)} \rightarrow 0$ as $\epsilon \rightarrow 0^{+}$, where $g_{\epsilon}$ is $-G(\cdot, z)$ with $\kappa$ replaced by $\kappa+i \epsilon$. Thus $u^{s}$ satisfies the above scattering problem if and only if $v$ satisfies the following Dirichlet problem, with $g$ given by (14) and $g_{\epsilon}$ defined as $-G(\cdot, z)$ with $\kappa$ replaced by $\kappa+i \epsilon$.

Problem 2 (BVP). Given $g, g_{\epsilon} L^{2}(\Gamma) \cap B C_{\infty}(\Gamma)$, for $\epsilon>0$, with $\left\|g_{\epsilon}-g\right\|_{L^{2}(\Gamma)} \rightarrow 0$ as $\epsilon \rightarrow 0$, find $v \in C^{2}(D) \cap C(\bar{D})$ which satisfies the Helmholtz equation (4) in $D$, the Dirichlet boundary condition $v=g$ on $\Gamma$, the bound (7), and the following limiting absorption principle: that, for all sufficiently small $\epsilon>0$, there exists $v_{\epsilon} \in C^{2}(D) \cap C(\bar{D})$ satisfying $v_{\epsilon}=g_{\epsilon}$ on $\Gamma$, (4) and (7), with $\kappa$ replaced by $\kappa+i \epsilon$, such that, for all $x \in D, v_{\epsilon}(x) \rightarrow v(x)$ as $\epsilon \rightarrow 0$.

We look for a solution to this boundary value problem as the combined singleand double-layer potential

$$
v(x):=u_{2}(x)-i \eta u_{1}(x), \quad x \in D,
$$

with some coupling parameter $\eta \geq 0$, where for a given function $\varphi \in L^{2}(\Gamma) \cap B C_{\infty}(\Gamma)$ we define the single-layer potential

$$
u_{1}(x):=\int_{\Gamma} G(x, y) \varphi(y) d s(y), \quad x \in \mathbb{R}^{3},
$$

and the double-layer potential

$$
u_{2}(x):=\int_{\Gamma} \frac{\partial G(x, y)}{\partial \nu(y)} \varphi(y) d s(y), \quad x \in \mathbb{R}^{3} .
$$


Seeking the solution of the boundary value problem in this form we will see that the boundary condition (14) is satisfied if and only if the BIE

$$
A \varphi=2 g
$$

holds on $\Gamma$, where

$$
A:=I+K-i \eta S
$$

and $I$ is the identity operator. Thus, the existence of a solution to the BVP is reduced to the study of the solvability of a boundary integral equation.

\section{General ideas and techniques}

Before we give an outline and emphasis the main results of the thesis we describe in some more detail the main ideas and techniques that are used for the forthcoming analysis. Among the many things the most important questions are:

- How to prove mapping properties of the operators?

- How to show the invertibility of the operator $A=I+K-i \eta S$ ?

- How to solve the integral equation numerically?

\subsection{How to prove mapping properties of the operators?}

In the case that the obstacle is smooth and bounded there is a well developed theory stating criteria on the kernel function to prove mapping properties of the corresponding integral operators between certain function spaces. The compactness of the scattering object is used in an essential way. For integral operators on noncompact manifolds some criteria can be found in the book of Jörgens [32], but they do not seem suited for the forthcoming analysis.

One result that is shown in Section 2.1 via Fourier techniques is that $S$ and $K$ are bounded operators e.g. on $L^{2}(\Gamma)$ and $L^{2}(\Gamma) \cap B C_{\infty}(\Gamma)$.

The main idea to prove this result is as follows: With the help of an appropriate positive cut-off function $\chi$ we define a partition of unity $\{\chi, 1-\chi\}$ to split the operators into a local and a global part. That is we write the integral operator $B$ with kernel function $b$, representing either the double- or single-layer potential operator, as the sum of two integral operators $B_{\text {global }}$ (the global part) and $B_{\text {local }}$ (the local part), with kernel functions

$$
b_{\text {global }}(x, y)=(1-\chi(|\boldsymbol{x}-\boldsymbol{y}|)) b(x, y)
$$

and

$$
b_{\text {local }}(x, y)=\chi(|\boldsymbol{x}-\boldsymbol{y}|) b(x, y), \quad x \neq y .
$$


Thus $B=B_{\text {global }}+B_{\text {local }}$ and we can study the mapping properties of $B_{\text {global }}$ and $B_{\text {local }}$ separately. This is very useful because we separated the local singularity from the singularity at infinity (non-integrability).

\section{The local part}

The local part has a weakly singular kernel. To prove that the corresponding operator maps into the space of continuous functions (or the space of continuous functions vanishing at infinity) one can adopt techniques used for bounded obstacles. Furthermore one can bound the kernel function by some convolution kernel so that mapping properties of convolution operators can be used to prove the mapping properties for Lebesgue spaces.

\section{The global part}

The global part has a continuous but non-integrable kernel. To treat this case we show, via Taylor expansion with respect to $x_{3}$ and $y_{3}$, that, for some small integer $N$,

$$
b_{\text {global }}(x, y)=\sum_{i=1}^{N} m_{i}(\boldsymbol{x}) \ell_{i}(\boldsymbol{x}-\boldsymbol{y}) n_{i}(\boldsymbol{y})+l(\boldsymbol{x}, \boldsymbol{y}),
$$

where $m_{i}, n_{i} \in B C\left(\mathbb{R}^{2}\right), \ell_{i} \in L^{2}\left(\mathbb{R}^{2}\right) \cap B C\left(\mathbb{R}^{2}\right)$. Furthermore the Fourier transform of $\ell_{i}$ is bounded, which we show via explicit computations. The remaining part of $b_{\text {global }}(x, y)$ after the finite sum is subtracted, namely $l(\boldsymbol{x}, \boldsymbol{y})$, is relatively wellbehaved, i.e. it is continuous and again bounded by an integrable convolution kernel. Thus the global part is written as sums and products of certain multiplication and convolution operators.

\subsection{How to show the invertibility of the operator $I+K-i \eta S$ ?}

It can be shown that the integral operators $S$ and $K$ are not compact, due to the non-compactness of the unbounded scattering surface. This lack of compactness deprives us of a very useful tool, the Riesz-Fredholm theory. In the classical case of smooth bounded obstacles this theory is used to prove the invertibility of the operator equation of the second kind, stating that for a compact perturbation of an invertible operator injectivity is equivalent to surjectivity.

To show that $A$ is indeed invertible we prove three essential steps:

\section{Step 1}

In the case of a flat surface the integral operators $S$ and $K$ are convolution operators, showing once more that they can not be compact due to their continuous spectrum. The invertibility of an convolution operator can be established via Fourier methods, i.e. by a characterisation of the spectrum of the operator. The equation (18) can be written as the convolution equation

$$
\psi(\boldsymbol{x})+\left(R_{h} * \psi\right)(\boldsymbol{x})=2 g(\boldsymbol{x}), \quad \boldsymbol{x} \in \mathbb{R}^{2},
$$


which is uniquely solvable if and only if

$$
1+\mathcal{F} R_{h}(\boldsymbol{x}) \neq 0, \quad \boldsymbol{x} \in \mathbb{R}^{2},
$$

where $R_{h}$ denotes the kernel of the combined double- and single-layer operator and $\mathcal{F}$ denotes the two-dimensional Fourier transform.

\section{Step 2}

We show that the integral operators depend continuously in the operator norm on variations of the boundary $\Gamma$. Together with the result from Step 1 this yields the invertibility in the case of a mildly rough surface.

\section{Step 3}

To prove the invertibility of the operator $A$ in the general case of an arbitrary rough surface we define a continuous deformation of a flat surface into the rough scattering surface, such that all intermediate surfaces have a uniformly bounded maximum surface slope. Starting with the invertibility result for the flat surface case, together with the result on the continuous dependence of $A$ on surface variations, we use explicitly known lower bounds on the norm of the operator $A$ that are uniform with respect to the maximum surface slope, to extend the invertibility over the whole range of the deformation.

\subsection{How to solve the integral equation numerically?}

The approximate numerical solution of the integral equation (18) is a challenging problem in scientific computing with a lot of open questions, both from a theoretical as well as a numerical point of view.

\section{Theoretical considerations}

From a theoretical point of view the main difficulty is the infinite integration domain. This makes it necessary to introduce an additional approximation step. One can interpret this approximation either as the truncation of the integral equation to some finite interval after which standard discretisation techniques for the case of finite intervals can be used or as the truncation of the infinite dimensional fully discretised linear system to a finite dimensional one. The convergence of this kind of truncation scheme can be analysed separately as it is independent of the convergence of the discretisation scheme. By far the most popular truncation scheme that is used in practical applications is the finite section method. For a large class of operators it is well understood whether this method is applicable, i.e. loosely speaking whether it converges for increasingly larger sections. We explain this method in some more detail in Section 6.3 and give references for further reading.

We have to point out that, to the author's understanding up to now, it is not known whether the already available criteria show the applicability of the finite section method to equation (18). In a recent paper [31] Lindner, Potthast and myself 
introduced a generalisation to the finite section, which has been termed multi-section method. For this scheme we were able to prove the convergence of the truncated solution to the solution of the equation (18). The main ideas of this method are summarised in Chapter 7.

\section{Numerical considerations}

From a computational point of view the main difficulties are the need to discretise very large surface patches, very often of the size of several hundred square wavelength. Following a rule of thumb that suggests to use at least 10 discretisation points per wavelength we have to solve linear systems for several hundred-thousands of unknowns. The discretisation of boundary integral operators generally yields a dense and unstructured linear system. To solve these systems the use of direct solvers is prohibitive on standard desktop machines with up to 4 GB RAM. Instead the method of choice will be to employ some iterative solver.

For such a problem the use of the banded matrix iterative algorithm (BMIA) has been suggested. This algorithm is based on a regular matrix splitting, where in each iteration step a sparse linear system has to be solved and one matrix-vector multiplication with a dense matrix has to be computed. The Nyström method that we are proposing in Chapter 6 yields a natural splitting that seems suitable for the BMIA algorithm. We explain the main ideas of the Nyström method and its interaction with the BMIA method in the following.

Originally, the Nyström method was introduced to solve integral equations of the second kind with continuous kernel functions. Under certain conditions on the kernel function it is possible to extend this method to the case of singular integral operators.

The reformulation of two-dimensional boundary value problems for the Laplace or Helmholtz equations with boundary integral equations yields integral operators, where the kernel functions can be decomposed in the form

$$
k(s, t)=k_{1}(s, t)+\ln |s-t| k_{2}(s, t), \quad s \neq t,
$$

with periodic smooth kernel functions $k_{1}$ and $k_{2}$. For this kind of situation it is possible to construct interpolatory quadrature formulas that incorporate the singular term directly into the quadrature weights, the original method dating back to [36]. These special quadrature rules give rise to high order Nyström methods. For more information on these kind of product quadratures that can be applied to solve boundary value problems for the Laplace and Helmholtz equation in 2D by means of boundary integral equations, we refer the reader to [35], [24], [23] and [4] and the citations therein.

A similar decomposition for our problem at hand could look like

$$
K(\boldsymbol{x}, \boldsymbol{y})=K_{1}(\boldsymbol{x}, \boldsymbol{y})+\frac{1}{|\boldsymbol{x}-\boldsymbol{y}|} K_{2}(\boldsymbol{x}, \boldsymbol{y}), \quad \boldsymbol{x} \neq \boldsymbol{y}
$$


The change in the type of singularity is not an essential problem. The more severe problem is that the function $K_{2}$ is no longer a smooth function. Instead we end up with a kernel function $K_{2}$ that has a diagonal discontinuity but is otherwise a bounded smooth function. Hence we see that it is not possible to construct a product integration rule in analogy to the two-dimensional case. Instead we follow an approach proposed in [12]. The main idea is to compute adjusted or locally corrected weights so that the underlying quadrature rule is again high order in the vicinity of the singularity. The corrected weights are given as the integral of the product of the singular function with some basis functions that approximate the density in a neighbourhood of the singularity. These integrals have to be computed to high precision by numerical integration. The original method in [12] suggested to compute these integrals by oversampling the region of integration until the result has converged to the desired accuracy. To reduce the amount of work for the numerical integration we apply a regularising change of variables. A change to polar coordinates removes the singularity completely. This observation is widely used in a series of integration schemes for the treatment of singular integrals. For example it is used in the floating partition of unity method introduced in [9].

The fully discretised system can be written in the form

$$
(\boldsymbol{I}+\boldsymbol{L}+\boldsymbol{G}) \boldsymbol{\psi}=\boldsymbol{g}
$$

where $\boldsymbol{\psi}$ and $\boldsymbol{g}$ denote vectors, containing the values of the unknown density and the right hand side at the set of integration points, $\boldsymbol{I}$ denotes the identity matrix, and $\boldsymbol{L}$ and $\boldsymbol{G}$ are matrices that represent the locally corrected weights and the far-field part of the integral operators. More precisely, the matrix $\boldsymbol{G}$ is a sparse matrix with band structure and $\boldsymbol{L}$ is a dense and unstructured matrix.

Following the idea of the BMIA method, we use the above decomposition to compute an approximate solution of (20), i.e. we compute

$$
\begin{aligned}
\boldsymbol{\psi}^{(0)} & :=\mathbf{0} \\
\boldsymbol{\psi}^{(n+1)} & :=(\boldsymbol{I}+\boldsymbol{L})^{-1}\left(\boldsymbol{g}-\boldsymbol{G} \boldsymbol{\psi}^{(n)}\right), \quad n=0,1,2, \ldots
\end{aligned}
$$

Thus, in each step we have to solve a linear system with a sparse banded matrix $\boldsymbol{I}+\boldsymbol{L}$ and do one matrix-vector multiplication with a dense unstructured matrix $\boldsymbol{G}$. The matrix $\boldsymbol{I}+\boldsymbol{L}$ is actually a block band matrix with band blocks so that again an iterative scheme, such as GMRES, can be used. However, the most time consuming step is the computation of the matrix-vector product $\boldsymbol{G} \boldsymbol{\psi}^{(n)}$. It is therefore inevitable to have a fast matrix-vector multiplication that reduces the overall cost from a standard $O\left(N^{2}\right)$ algorithm, where $N$ denotes the total number of unknowns.

A method called the canonical grid method has been proposed to deal with these special kind of matrix-vector product that arise in rough surface scattering problems. The method consists in finding a clever approximation to the kernel function of the boundary integral operators that allow the use of fast Fourier methods. Thus the 
cost for on matrix-vector multiplication can be reduced to $O(M \cdot N \log N)$, where $M$ denotes a usually small number that depends only on the height of the object measured in terms of the wavenumber.

\section{Thesis outline}

For the rest of this thesis it is very useful to have a short hand notation for the following Banach spaces:

$$
X(\Gamma):=L^{2}(\Gamma) \cap B C(\Gamma) \quad \text { and } \quad X_{\infty}(\Gamma):=L^{2}(\Gamma) \cap B C_{\infty}(\Gamma) .
$$

The thesis consists of two parts. Part I concerns the development of a framework that allows to prove the invertibility of the integral operator of the second kind that arises from the treatment of rough surface scattering problems in three dimensions by means of BIEs. Part II deals with ideas for the effective numerical solution of the underlying BIE and ways to prove convergence results for the discretised BIE.

\section{Part I}

The results in the first three chapters that concern mapping properties and the invertibility of the operator $A$, given through (19), in the algebra of all bounded linear operators on $L^{2}(\Gamma)$ and $X(\Gamma)$, denoted by $B L\left(L^{2}(\Gamma)\right)$ and $B L(X(\Gamma))$, have been published in joint papers together with S.N. Chandler-Wilde and R. Potthast, see [21], [22] and [20]. These results are summarised and form the basis to prove mapping properties and invertibility results in the space

$$
X_{\infty}(\Gamma):=L^{2}(\Gamma) \cap B C_{\infty}(\Gamma)
$$

This space is a closed subspace of $X(\Gamma)$ consisting of functions that have at least a qualitative decay behaviour.

\section{Chapter 1}

Necessary tools from functional analysis as well as results from Fourier analysis are reviewed. Several important Lemmata on mapping properties of convolution and multiplication operators are shown.

\section{Chapter 2}

Mapping properties of the single- and double-layer potential operators are analysed. The standard jump relations for single- and double-layer boundary potentials over bounded obstacles are extended to the case of a $2 \mathrm{D}$ rough surface. The continuous dependence of the boundary operators with respect to variations of the boundary and with respect to the wavenumber are shown. 


\section{Chapter 3}

The uniqueness of the BVP is proven as well as the equivalence of the BVP with the integral equation. Furthermore it is shown that if $A$ is invertible in the Banach algebra $B L\left(L^{2}(\Gamma)\right)$ then it is also invertible in the subalgebras $B L(X(\Gamma))$ and $B L\left(X_{\infty}(\Gamma)\right)$.

We next study the invertibility of the operator $I+K-i \eta S$, firstly for the case when $\Gamma$ is flat and the operator $I+K-i \eta S$ is a convolution operator, and then for the general case when $\Gamma$ is rough. Starting from the above results we show that $A$ is invertible on $L^{2}(\Gamma)$, without restriction on the surface elevation or slope of $\Gamma$.

\section{Part II}

\section{Chapter 4}

In this chapter we introduce notation used throughout the second part of the thesis, summarise results from Fourier analysis for multi-periodic functions and sequences. We prove that the composite trapezoidal rule is a high order integration scheme on $\mathbb{R}^{d}$ for a class of differentiable and sufficiently fast decaying functions thus extending results shown in [40] and [42].

\section{Chapter 5}

We study operator approximations used in Nyström methods for the weakly singular integral operators occurring in the rough surface scattering problem. The operators are split into a global smooth part and a local weakly singular part. In the case that the density is smooth and sufficiently fast decaying we prove that the operator approximations exhibit pointwise convergence that is of super-algebraic convergence order. The results we show for the global operator extend ideas from [40] and [42] to the three-dimensional case. The treatment of the weakly singular operators follows ideas from [12] and [9] that were originally used for bounded obstacles.

\section{Chapter 6}

We present ideas how to apply a Nyström method to the case of integral equations of the second kind over unbounded domains. We try to motivate the need of an additional truncation scheme and introduce the finite section method. Though the solvability of the truncated fully discretised equation is not known, a lot of numerical schemes have been developed for the efficient numerical solution of these large linear systems. We give a short introduction to the BMIA, for which we suggest a new matrix splitting that arises naturally from our operator approximation.

\section{Chapter 7}

As an alternative approach to the finite section method we give a short introduction to the Multi-section method that was developed in [31]. For this scheme the convergence can be shown and we summarise the main results. A numerical realisation of 
this truncation scheme leads to an approximative matrix equation that is solved in a least squares sense. This can be done with the help of an iterative scheme, e.g. the conjugate gradient method for linear least squares problems (CGLS). The operator discretisation that we introduced in Chapter 5 can also be utilised for this scheme.

\section{Chapter 8}

Whether one uses the BMIA or the CGLS method to compute a solution of the truncated equation, it is necessary to utilise fast matrix-vector multiplication schemes. A scheme that was especially designed to handle the dense large matrices is the canonical grid method (CGM) that we introduce in Chapter 9. As a preparation for the CGM we explain the use of fast matrix-vector multiplication algorithms by means of fast Fourier transformation (FFT) for integral operators with difference kernels. The theory and algorithms are developed for arbitrary space dimensions and we give an example, including a full MATLAB listing for a two-dimensional problem.

\section{Chapter 9}

We present the classical CGM based on Taylor expansion, cf. [45] and [62], and another variant based on interpolation, cf. [19], with Chebyshev polynomials. Both methods emphasise the two-dimensional convolutional structure of the integral operator. We introduce a novel approach that emphasises a three-dimensional convolutional structure of the potentials rather than the potential operators. To see this three-dimensional convolution we write the potential in terms of a singular surface measure which can be realised on a formal level by introducing a Dirac delta distribution. For practical computations we replace the Dirac delta distribution by a sequence of continuous functions that form a Dirac delta sequence. Following an error analysis in [5] we derive criteria, the so called discrete moment conditions, that ensure that the Dirac delta sequence mimics the sifting property of the Dirac delta distribution on a discrete level for all polynomials up to a certain degree. These approximations to the Dirac delta distribution define high order local interpolation schemes. Starting from the discrete moment conditions we use ideas from [46] to derive a linear system of algebraic equations that define the interpolation kernels in terms of a piecewise polynomial. We prove error estimates for these high order interpolation schemes, give some numerical example and reinterpret this method as a classical CGM based on a special choice for the interpolation operator. We conclude the chapter with some ideas from the theory of radial basis functions (RBFs) that can serve as an alternative kernel function for the local high order interpolation scheme.

\section{Chapter 10}

In the last chapter we give a full listing of a possible MATLAB implementation of the novel algorithm. This serves two aims: first of all we hope that the details of the algorithm on the fully discrete level can be better understood if shown in a few lines 
of MATLAB code. Second, we want to emphasise the simplicity of the algorithm as compared to alternatives like fast multipole methods, equivalent sources methods or precorrected FFT methods, though at the same time it is highly efficient. 
Part I

Theory 



\section{Chapter 1}

\section{Tools}

This chapter reviews results from functional and Fourier analysis and introduces the notation for function spaces used throughout this thesis.

\subsection{Results from functional analysis}

The results summarised in the upcoming sections are classical results that can be found in any functional analysis books, e.g. [58], [48] and [60]. They are included mainly to make the thesis selfcontained as far as possible and to have the notation and exact definitions at hand.

\subsubsection{Some results on bounded linear mappings}

For normed spaces $\left(X,\|\cdot\|_{X}\right)$ and $\left(Y,\|\cdot\|_{Y}\right)$ the product space $X \times Y$ is a normed space equipped with the norm $\|(x, y)\|_{X \times Y}:=\|x\|_{X}+\|y\|_{Y}$. Convergence in $X \times Y$ is equivalent to componentwise convergence, i.e. the norm $\|\cdot\|_{X \times Y}$ is equivalent to $\|(x, y)\|_{X \times Y, \infty}:=\max \left(\|x\|_{X},\|y\|_{Y}\right)$. If $X$ and $Y$ are Banach spaces so is $X \times Y$.

In the case that $X$ and $Y$ have a non-empty intersection $X \cap Y$ is a normed spaces equipped with the norm $\|z\|_{X \cap Y}:=\max \left(\|z\|_{X},\|z\|_{Y}\right)$. If $X$ and $Y$ are Banach spaces so is $X \cap Y$.

The space of all continuous linear operators from $X$ to $Y$ is denoted by $B L(X ; Y)$; in the case $X=Y$ we write shortly $B L(X):=B L(X ; X)$. It is well known that a linear operator is continuous if and only if it is bounded. Furthermore $B L(X ; Y)$ is a normed space equipped with the operator norm

$$
\|A\|_{X \rightarrow Y}:=\sup _{\|x\|_{X}=1}\|A x\|_{Y}, \quad A \in B L(X, Y) .
$$

If the spaces are evident from the context we write shortly $\|A\|$ instead of $\|A\|_{X \rightarrow Y}$. If $Y$ is a Banach space then so is $B L(X ; Y)$.

The set of all invertible operators in $B L(X)$ is an open set and denoted by $G L(X)$.

The following theorem is often used to extend the domain of definition of an linear operator defined on a dense subspace to its closure. 
TheOrem 1.1 (The linear extension theorem). Let $X$ be a normed space, $X_{0}$ some dense subspace of $X$ and $Z$ a Banach space. Let $A: X_{0} \rightarrow Z$ be a bounded linear operator. Then there exists a unique extension of $A$ to a bounded linear operator $\bar{A}: X \rightarrow Z$ with $\|A\|=\|\bar{A}\|$.

Proof. The main idea of the proof is to define the extension is through

$$
\bar{A} \varphi:=\lim _{n \rightarrow \infty} A \varphi_{n}, \quad \text { for } \varphi \in X,
$$

where $\left(\varphi_{n}\right)_{n \in \mathbb{N}}$ is some approximating sequence of function in $X_{0}$ with limit $\varphi$. For a detailed proof see e.g. [58, Satz 2.19].

\subsubsection{Some classical function spaces}

This section briefly introduces the notation for the most frequently used spaces.

Spaces of continuous functions The space of bounded and continuous real- or complex-valued functions on $\mathbb{R}^{d}$ is denoted by $B C\left(\mathbb{R}^{d}\right)$. The space of bounded, continuous, real- or complex-valued functions vanishing at infinity is denoted by $B C_{\infty}\left(\mathbb{R}^{d}\right)$, i.e.

$$
B C_{\infty}\left(\mathbb{R}^{d}\right):=\left\{\varphi \in B C\left(\mathbb{R}^{d}\right): \lim _{|x| \rightarrow \infty} \varphi(x)=0\right\} .
$$

The spaces $B C\left(\mathbb{R}^{d}\right)$ and $B C_{\infty}\left(\mathbb{R}^{d}\right)$ equipped with the supremum norm

$$
\|\varphi\|_{B C\left(\mathbb{R}^{d}\right)}:=\sup _{x \in \mathbb{R}^{d}}|\varphi(x)|
$$

are Banach spaces. $B C_{\infty}\left(\mathbb{R}^{d}\right)$ is a closed subspace of $B C\left(\mathbb{R}^{d}\right)$ arginparA more unique notation would be better We will use

Spaces of differentiable functions To define spaces of differentiable functions the multi-index notation is applied.

Definition 1.2 (Multi-index notation). A multi-index $\alpha \in \mathbb{N}_{0}^{d}$ is a d-tuple of nonnegative integers together with the following set of rules: for $\alpha=\left(\alpha_{1}, \ldots, \alpha_{d}\right), \beta=$ $\left(\beta_{1}, \ldots, \beta_{d}\right) \in \mathbb{N}_{0}^{d}$ and $x \in \mathbb{R}^{d}$ we define

$$
\begin{aligned}
\alpha \leq \beta & : \Leftrightarrow \quad \alpha_{1} \leq \beta_{1}, \ldots, \alpha_{d} \leq \beta_{d}, \\
\alpha+\beta & :=\left(\alpha_{1}+\beta_{1}, \ldots, \alpha_{d}+\beta_{d}\right), \\
|\alpha| & :=\alpha_{1}+\cdots+\alpha_{d}, \quad \text { the order of a multi-index } \\
\alpha ! & :=\alpha_{1} ! \ldots \alpha_{d} ! \\
x^{\alpha} & :=x_{1}^{\alpha_{1}} \ldots x_{d}^{\alpha_{d}} .
\end{aligned}
$$


Thus, for a function $\varphi: \mathbb{R}^{d} \rightarrow \mathbb{C}$ and multi-index $\alpha \in \mathbb{N}_{0}^{d}$ we can write $\partial^{\alpha} \varphi$ for the partial derivatives

$$
\left(\frac{\partial}{\partial x_{1}}\right)^{\alpha_{1}} \cdots\left(\frac{\partial}{\partial x_{d}}\right)^{\alpha_{d}} \varphi(x)
$$

For any integer $k \in \mathbb{N}_{0}$ we set

$$
\begin{aligned}
C^{k}\left(\mathbb{R}^{d}\right) & :=\left\{\varphi: \partial^{\alpha} \varphi \in C\left(\mathbb{R}^{d}\right) \text { for }|\alpha| \leq k\right\}, \\
B C^{k}\left(\mathbb{R}^{d}\right) & :=\left\{\varphi: \partial^{\alpha} \varphi \in B C\left(\mathbb{R}^{d}\right) \text { for }|\alpha| \leq k\right\},
\end{aligned}
$$

the space of real- or complex-valued functions on $\mathbb{R}^{d}$ with $k$ continuous (continuous and bounded) derivatives.

$$
C^{\infty}\left(\mathbb{R}^{d}\right):=\bigcap_{k=1}^{\infty} C^{k}\left(\mathbb{R}^{d}\right) \text { and } B C^{\infty}\left(\mathbb{R}^{d}\right):=\bigcap_{k=1}^{\infty} B C^{k}\left(\mathbb{R}^{d}\right),
$$

the space of smooth (bounded and smooth), real- or complex-valued functions on $\mathbb{R}^{d}$.

$$
C_{\text {comp }}^{k}\left(\mathbb{R}^{d}\right):=\left\{\varphi \in C^{k}\left(\mathbb{R}^{d}\right) \mid \operatorname{supp} \varphi \subset \mathbb{R}^{d} \text { is compact }\right\},
$$

the space of $k$-times continuously differentiable, real- or complex-valued functions with compact support on $\mathbb{R}^{d}$.

$$
\mathcal{D}\left(\mathbb{R}^{d}\right):=C_{\mathrm{comp}}^{\infty}\left(\mathbb{R}^{d}\right):=\bigcap_{k=1}^{\infty} C_{\mathrm{comp}}^{k}\left(\mathbb{R}^{d}\right)
$$

the space of test functions.

$$
\mathcal{S}\left(\mathbb{R}^{d}\right):=\left\{\varphi \in C^{\infty}\left(\mathbb{R}^{d}\right)\left|\forall m \in \mathbb{N}_{0}, \beta \in \mathbb{N}_{0}^{d}: \sup _{x \in \mathbb{R}^{d}}\left(1+|x|^{m}\right)\right| \partial^{\beta} f(x) \mid<\infty\right\},
$$

the Schwartz space of rapidly decreasing functions. It is obvious that the following inclusion holds

$$
\mathcal{D}\left(\mathbb{R}^{d}\right) \subset \mathcal{S}\left(\mathbb{R}^{d}\right)
$$

Spaces of Hölder continuous functions A real- or complex-valued function $\varphi$ defined on $\mathbb{R}^{d}$ is called uniformly Hölder continuous with Hölder exponent $0<\alpha \leq 1$ if there exists a constant $C>0$ such that

$$
|\varphi(x)-\varphi(y)| \leq C|x-y|^{\alpha}
$$

for all $x, y \in \mathbb{R}^{d}$. By $B C^{0, \alpha}\left(\mathbb{R}^{d}\right)$ we denote the space of all functions that are bounded and uniformly Hölder continuous with exponent $\alpha$.

$B C^{0, \alpha}\left(\mathbb{R}^{d}\right) \subset B C\left(\mathbb{R}^{d}\right)$ is called a Hölder space. It is a Banach space equipped with the norm

$$
\|\varphi\|_{B C^{0, \alpha}}:=\|\varphi\|_{B C\left(\mathbb{R}^{d}\right)}+|\varphi|_{\alpha, \mathbb{R}^{d}}
$$


where

$$
|\varphi|_{\alpha, \mathbb{R}^{d}}:=\sup _{\substack{x, y \in \mathbb{R}^{d} \\ x \neq y}} \frac{|\varphi(x)-\varphi(y)|}{|x-y|^{\alpha}}
$$

denotes the Hölder semi-norm. $|\varphi|_{\alpha, \mathbb{R}^{d}}$ is the smallest constant satisfying (1.2) and named Hölder constant of $\varphi$.

REMARK 1.3. In the case $\alpha=1$ the function is called Lipschitz continuous. A uniformly Hölder or Lipschitz continuous function is uniformly continuous.

We also need spaces of bounded continuously differentiable functions that have bounded and uniformly Hölder continuous first derivatives. This space is denoted by $B C^{1, \alpha}\left(\mathbb{R}^{d}\right)$. Among the many equivalent norms on $B C^{1, \alpha}\left(\mathbb{R}^{d}\right)$ we choose

$$
\|\varphi\|_{B C^{1, \alpha}}:=\|\varphi\|_{B C\left(\mathbb{R}^{d}\right)}+\sup _{x \in \mathbb{R}^{d}}|\nabla \varphi(x)|+\sup _{\substack{x, y \in \mathbb{R}^{d} \\ x \neq y}} \frac{|\nabla \varphi(x)-\nabla \varphi(y)|}{|x-y|^{\alpha}} .
$$

The space $B C^{1, \alpha}\left(\mathbb{R}^{d}\right) \subset B C^{1}\left(\mathbb{R}^{d}\right)$ equipped with the norm $\|\cdot\|_{B C^{1, \alpha}}$ is a Banach space.

Spaces of Lebesgue integrable functions For $1 \leq p<\infty$ we denote by $L^{p}\left(\mathbb{R}^{d}\right)$ the linear space of equivalence classes of real- or complex-valued Lebesgue measurable functions $\varphi$ on $\mathbb{R}^{d}$ such that $|\varphi|^{p}$ is integrable over $\mathbb{R}^{d}$. As it is the usual custom two functions are identified if they agree except for a set of measure zero which is written shortly as a.e. for almost everywhere, i.e. if for two measurable functions defined a.e.

$$
f: \mathbb{R}^{d} \backslash N_{f} \rightarrow \mathbb{K}, \quad g: \mathbb{R}^{d} \backslash N_{g} \rightarrow \mathbb{K},
$$

the sets $N_{f}, N_{g}$ and $\left\{x \in \mathbb{R}^{d} \backslash\left(N_{f} \cup N_{g}\right): f(x) \neq g(x)\right\}$ are of measure zero. One can show that all functions in one equivalence class are integrable and have the same integral. On the set of equivalence classes

$$
\|\varphi\|_{L^{p}\left(\mathbb{R}^{d}\right)}:=\left(\int_{\mathbb{R}^{d}}|\varphi(x)|^{p} d x\right)^{1 / p} .
$$

defines a norm which is called the $L^{p}$-norm.

Theorem 1.4 (Riesz-Fischer theorem for $1 \leq p<\infty$ ). For $1 \leq p<\infty$ the spaces $L^{p}\left(\mathbb{R}^{d}\right)$ equipped with $\|\cdot\|_{L^{p}\left(\mathbb{R}^{d}\right)}$ are Banach spaces. For every convergent sequence there exists a subsequence that converges pointwise almost everywhere.

The linear space of equivalence classes of measurable functions that are essentially bounded is denoted by $L^{\infty}\left(\mathbb{R}^{d}\right)$, i.e. a measurable function $\varphi: \mathbb{R}^{d} \rightarrow \mathbb{K}$ belongs to 
$L^{\infty}\left(\mathbb{R}^{d}\right)$ if

$$
\|\varphi\|_{L^{\infty}\left(\mathbb{R}^{d}\right)}:=\text { ess. } \sup |\varphi(x)|:=\inf \{C \in \mathbb{R}:|\varphi(x)| \leq C \text { a.e. }\}<\infty .
$$

One can show that $\|\varphi\|_{L^{\infty}\left(\mathbb{R}^{d}\right)}$ defines a norm on $L^{\infty}\left(\mathbb{R}^{d}\right)$.

THEOREM 1.5 (Riesz-Fischer theorem for $p=\infty$ ). The spaces $L^{\infty}\left(\mathbb{R}^{d}\right)$ equipped with $\|\cdot\|_{L^{\infty}\left(\mathbb{R}^{d)}\right)}$ is a Banach spaces. For every convergent sequence there exists a subsequence that converges pointwise almost everywhere.

REMARK 1.6. Following the usual custom we talk of $L^{p}$-functions instead of equivalence classes of functions. This is a reasonable approach as long as one does not try to use pointwise properties of $L^{p}$-functions. One exception is the case that $f \in L^{p}$ equals to a continuous function a.e.. As one can show that there can only be one continuous function in one equivalence class, one chooses this functions as representative.

To prove mapping properties of convolution operators on $L^{p}$-spaces in Section 1.5 we need the Hölder inequality. It is used also to prove the Minkowski inequality, i.e. to show that (1.3) actually is a norm.

In dealing with $L^{p}$-spaces it is useful to define conjugate pairs of exponents, i.e. for $p \in[1, \infty]$ let $p^{\prime}$ denote the conjugate exponent such that

$$
\frac{1}{p}+\frac{1}{p^{\prime}}=1
$$

where one uses the convention $\frac{1}{\infty}=0$.

TheOREM 1.7 (Hölder inequality). Let $1 \leq p \leq \infty$. For $f \in L^{p}\left(\mathbb{R}^{d}\right), g \in L^{p^{\prime}}\left(\mathbb{R}^{d}\right)$ it holds that $f g \in L^{1}\left(\mathbb{R}^{d}\right)$ and

$$
\|f g\|_{L^{1}} \leq\|f\|_{L^{p}}\|g\|_{L^{p^{\prime}}}
$$

or written as integral inequality

$$
\int_{\mathbb{R}^{d}}|f(x) g(x)| d x \leq\left(\int_{\mathbb{R}^{d}}|f(x)|^{p} d x\right)^{1 / p}\left(\int_{\mathbb{R}^{d}}|g(x)|^{p^{\prime}} d x\right)^{1 / p^{\prime}} .
$$

Proof. For a proof see [58, Satz 1.39].

An easy consequence is the

COROLlary 1.8 (Generalised Hölder inequality). Let $1 \leq p, q, r \leq \infty$ with $\frac{1}{r}=$ $\frac{1}{p}+\frac{1}{q}$. For $f \in L^{p}\left(\mathbb{R}^{d}\right), g \in L^{q}\left(\mathbb{R}^{d}\right)$ it holds that $f g \in L^{r}\left(\mathbb{R}^{d}\right)$ and

$$
\|f g\|_{L^{r}} \leq\|f\|_{L^{p}}\|g\|_{L^{q}}
$$


or written as integral inequality

$$
\left(\int_{\mathbb{R}^{d}}|f(x) g(x)|^{r} d x\right)^{1 / r} \leq\left(\int_{\mathbb{R}^{d}}|f(x)|^{p} d x\right)^{1 / p}\left(\int_{\mathbb{R}^{d}}|g(x)|^{q} d x\right)^{1 / q} .
$$

Proof. We note that

$$
|f g|^{r}=|f|^{r}|g|^{r} \in L^{1} \quad \Longleftrightarrow \quad|f g|=|f||g| \in L^{r}
$$

and

$$
\frac{1}{r}=\frac{1}{p}+\frac{1}{q} \Longleftrightarrow 1=\frac{1}{\frac{p}{r}}+\frac{1}{\frac{q}{r}}
$$

Thus we can apply the ordinary Hölder inequality (THEOREM 1.7) and compute

$$
\int|f g|^{r} d x=\int|f|^{r}|g|^{r} d x \leq\left(\int|f|^{r \cdot p / r} d x\right)^{r / p}\left(\int|g|^{r \cdot q / r} d x\right)^{r / q}
$$

Taking the $r$ th root on both sides finishes the proof.

In the case that the Lebesgue spaces are defined over some bounded domain $\Omega \subset \mathbb{R}^{d}$ it is a consequence of the Hölder inequality that for $1<p<q \leq \infty$ it holds that

$$
\|f\|_{L^{p}(\Omega)} \leq|\Omega|^{\frac{1}{p}-\frac{1}{q}}\|f\|_{L^{q}(\Omega)}
$$

for all $f \in L^{q}(\Omega)$, i.e. $L^{q}(\Omega) \subset L^{p}(\Omega)$. In the case that $|\Omega|=\infty$ in general neither of the inclusions $L^{q}\left(\mathbb{R}^{d}\right) \subset L^{p}\left(\mathbb{R}^{d}\right)$ nor $L^{p}\left(\mathbb{R}^{d}\right) \subset L^{q}\left(\mathbb{R}^{d}\right)$ are valid.

For any two numbers $p, q$ with $1 \leq p<s<q \leq \infty$ it holds that a function from the intersection of $L^{p}$ and $L^{q}$ also belongs to any $L^{s}$-space for $p<s<q$. This is a very elementary example of a so called interpolation theorem, sometimes called Lyapunov inequality cf. [60, Lemma II.4.1].

Lemma 1.9. Let $1 \leq p \leq q \leq \infty$ and $0 \leq \theta \leq 1$. Define $s$ through $\frac{1}{s}=(1-\theta) \frac{1}{p}+\theta \frac{1}{q}$, then $L^{p}\left(\mathbb{R}^{d}\right) \cap L^{q}\left(\mathbb{R}^{d}\right) \subset L^{s}\left(\mathbb{R}^{d}\right)$ and

$$
\|f\|_{L^{s}} \leq\|f\|_{L^{p}}^{1-\theta}\|f\|_{L^{q}}^{\theta} \quad \text { for all } f \in L^{p}\left(\mathbb{R}^{d}\right) \cap L^{q}\left(\mathbb{R}^{d}\right) .
$$

Proof. We note that $1=\frac{(1-\theta) s}{p}+\frac{(\theta) s}{q}$ and with the help of the Hölder inequality compute

$$
\int|f|^{s} d x=\int|f|^{(1-\theta) s}|f|^{\theta s} d x \leq\left(\int|f|^{(1-\theta) s \cdot \frac{p}{(1-\theta) s}} d x\right)^{\frac{(1-\theta) s}{p}}\left(\int|f|^{\theta s \cdot \frac{q}{(\theta s}} d x\right)^{\frac{\theta s}{q}} .
$$

Taking the $s$-th root on both sides finishes the proof. 
The following example illustrates the preceding Lemma. We use it later on to show the mapping properties of the boundary operators.

EXAmple 1.10. Consider the function on $\mathbb{R}^{2}$ given through

$$
\ell(\boldsymbol{y}):= \begin{cases}|\boldsymbol{y}|^{\alpha-2}, & |\boldsymbol{y}| \leq 1 \\ 0, & |\boldsymbol{y}|>1,\end{cases}
$$

for some $\alpha \in(0,1]$. Changing to polar coordinates one computes that

$$
\int_{\mathbb{R}^{2}}|\ell(\boldsymbol{y})|^{p} d \boldsymbol{y}=\int_{B_{1}(0)}|\ell(\boldsymbol{y})|^{p} d \boldsymbol{y}=2 \pi \int_{0}^{1} r^{(\alpha-2) p} r d r<\infty
$$

for all $p \in\left[1, \frac{2}{2-\alpha}\right)$, i.e. $\ell \in L^{1}\left(\mathbb{R}^{2}\right) \cap L^{p}\left(\mathbb{R}^{2}\right)$ for $1 \leq p<\frac{2}{2-\alpha}<2$.

For later use it is important to know dense subspaces of Lebesgue spaces. One can show

LEMMA 1.11. For $1 \leq p<\infty$ the space $\mathcal{D}\left(\mathbb{R}^{d}\right)$ is a dense subspace of $L^{p}\left(\mathbb{R}^{d}\right)$, i.e. for each $\varphi \in L^{p}\left(\mathbb{R}^{d}\right)$ there exists a sequence of functions $\left(\varphi_{n}\right)_{n \in \mathbb{N}}$ in $\mathcal{D}\left(\mathbb{R}^{d}\right)$ such that $\|\varphi-\varphi\|_{L^{p}\left(\mathbb{R}^{d}\right)} \rightarrow 0$ for $n \rightarrow \infty$.

As an immediate consequence of the inclusion (1.1) we get:

Lemma 1.12. For $1 \leq p<\infty$ the space $\mathcal{S}\left(\mathbb{R}^{d}\right)$ is a dense subspace of $L^{p}\left(\mathbb{R}^{d}\right)$.

Among the Lebesgue space the case $p=2$ deserves some extra attention as it is the only $L^{p}$-space that is a Hilbert space. The scalar product is given in the usual way by

$$
(\varphi, \psi)_{L^{2}\left(\mathbb{R}^{d}\right)}:=\int_{\mathbb{R}^{d}} \varphi(x) \overline{\psi(x)} d x
$$

for $\varphi, \psi \in L^{2}\left(\mathbb{R}^{d}\right)$ and we see that the Cauchy-Schwarz inequality is just a special case of the Hölder inequality, i.e.

$$
\left|(\varphi, \psi)_{L^{2}}\right| \leq\|\varphi \psi\|_{L^{1}} \leq\|\varphi\|_{L^{2}}\|\psi\|_{L^{2}}
$$

Radial functions For later use in connection with symmetry properties of Fourier transformation it is useful to introduce the concept of radial functions in $L^{p}$-spaces. A function $f: \mathbb{R}^{d} \rightarrow \mathbb{K}$ is said to be radial if it can be written in the form

$$
f(x)=f_{\text {rad }}(|x|), \quad x \in \mathbb{R}^{d},
$$

where $f_{\text {rad }}: \mathbb{R}_{\geq 0} \rightarrow \mathbb{C}$. It is clear that a function is radial if and only if

$$
f(x)=f(Q x), \quad \text { for all } x \in \mathbb{R}^{d} \text { and } Q \in O\left(\mathbb{R}^{d}\right),
$$


where $O\left(\mathbb{R}^{d}\right)$ denotes the group of orthogonal transformations on $\mathbb{R}^{d}$. If $f \in L^{p}\left(\mathbb{R}^{2}\right)$ is a radial function it follows from Fubini's theorem together with a change of variables $x=r(\cos \theta, \sin \theta)$ that

$$
\|f\|_{L^{p}}^{p}=\int_{\mathbb{R}^{2}}|f(\boldsymbol{x})|^{p} d \boldsymbol{x}=\int_{0}^{\infty} \int_{0}^{2 \pi}\left|f_{\mathrm{rad}}(r)\right|^{p} d \theta r d r=2 \pi \int_{0}^{\infty}\left|f_{\mathrm{rad}}(r)\right|^{p} r d r<\infty,
$$

i.e. the radial part of $f$ is an element of $L^{p}\left(\mathbb{R}_{\geq 0}, r d r\right)$, the weighted $L^{p}$-space on the positive half-line.

\subsubsection{Function spaces on the boundary}

The scattering surface $\Gamma$ given by (1) has the natural parametrisation

$$
P_{f}: \mathbb{R}^{2} \rightarrow \mathbb{R}^{3}, \quad \boldsymbol{x} \mapsto(\boldsymbol{x}, f(\boldsymbol{x})) .
$$

We can use this parametrisation to define the counterparts of the previously introduced functions spaces for functions living on the boundary.

In the case of continuous or continuously differentiable functions we define

$$
\varphi \in B C^{k}(\Gamma) \quad: \Longleftrightarrow \quad \varphi \circ P_{f} \in B C^{k}\left(\mathbb{R}^{2}\right), \quad \text { for } k \in \mathbb{N}_{0} .
$$

The right hand side is meaningful if we assume that $P_{f} \in B C^{k}\left(\mathbb{R}^{2}, \mathbb{R}^{3}\right)$ for some $k \in \mathbb{N}$. Thus in general the functions defined on the scattering surface can not be any smoother than the scattering surface itself. It is clear that this definition extends in the obvious way to Hölder spaces, etc.. Furthermore we conclude that the mapping

$$
I_{f}: B C^{n, \alpha}\left(\Gamma_{f}\right) \rightarrow B C^{n, \alpha}\left(\mathbb{R}^{2}\right), \quad\left(I_{f} \varphi\right)(\boldsymbol{x}):=\left(\varphi \circ P_{f}\right)(\boldsymbol{x}), \quad \boldsymbol{x} \in \mathbb{R}^{2},
$$

defines an isomorphism for $n \in \mathbb{N}, \alpha \in[0,1]$.

For $L^{p}$-functions we note that the Lebesgue integral over the scattering surface $\Gamma$ is defined through

$$
\int_{\Gamma}|\varphi(y)|^{p} d s(y):=\int_{\mathbb{R}^{2}}\left|\left(\varphi \circ P_{f}\right)(\boldsymbol{y})\right|^{p} J(\boldsymbol{y}) d \boldsymbol{y},
$$

where

$$
J(\boldsymbol{y}):=\sqrt{1+|\nabla f(\boldsymbol{y})|^{2}}, \quad \boldsymbol{y} \in \mathbb{R}^{d},
$$

denotes the surface area-element. For $f \in B C^{1}\left(\mathbb{R}^{2}\right)$ we have the bounds

$$
1 \leq J(\boldsymbol{y}) \leq \sqrt{1+L_{f}^{2}}=: \tilde{L}
$$


where

$$
L_{f}:=\sup _{\substack{\boldsymbol{x}, \boldsymbol{y} \in \mathbb{R}^{2} \\ \boldsymbol{x} \neq \boldsymbol{y}}} \frac{|f(\boldsymbol{x})-f(\boldsymbol{y})|}{|\boldsymbol{x}-\boldsymbol{y}|}=\sup _{\boldsymbol{x} \in \mathbb{R}^{2}}|\nabla f(\boldsymbol{x})|,
$$

denotes the Lipschitz constant of $f$, i.e. the maximum surface slope. It follows from Hölder's inequality and the bound (1.11) that

$$
\begin{aligned}
& \varphi \in L^{p}(\Gamma, d s) \\
\Longleftrightarrow & \varphi \circ P_{f} \in L^{p}\left(\mathbb{R}^{2}, J(\boldsymbol{y}) d \boldsymbol{y}\right) \\
\Longleftrightarrow & \varphi \circ P_{f} \in L^{p}\left(\mathbb{R}^{2}\right) .
\end{aligned}
$$

Thus we see that the mapping given through (1.9) defines an isomorphism for all of the before mentioned spaces, including the Lebesgue spaces, provided that the surface is sufficiently smooth for the above definitions to be meaningful. In this way all results from the previous sections, including the denseness statements, transfer to the spaces on the boundary. Furthermore we can use this isomorphism to associate to each operator $A_{f} \in B L\left(Z\left(\Gamma_{f}\right)\right)$ an operator

$$
\tilde{A}_{f}:=I_{f} A_{f} I_{f}^{-1} \in B L\left(\mathcal{Z}\left(\mathbb{R}^{2}\right)\right),
$$

where $\mathcal{Z} \in\left\{B C_{\infty}^{k, \alpha}, \mathcal{S}, \mathcal{D}, L^{p}\right\}$ stands for any of the previous spaces.

\subsubsection{Integral operators over unbounded domains}

The following two Lemmata are a slight generalisation of the well known theorems for integral operators with continuous and weakly singular kernels on bounded domains. The following proofs are adopted from [35, Theorem 2.21, and Theorem 2.22].

Lemma 1.13. Consider the integral operator

$$
(A \psi)(x):=\int_{\mathbb{R}^{d}} K(x, y) \psi(y) d y, \quad x \in \mathbb{R}^{d}
$$

where $K: \mathbb{R}^{d} \times \mathbb{R}^{d} \rightarrow \mathbb{C}$ denotes a bounded, continuous function with compact support, i.e. there exist positive constants $C$ and $R$ such that

$$
\sup _{(x, y) \in \mathbb{R}^{d} \times \mathbb{R}^{d}}|K(x, y)| \leq C
$$

and

$$
\operatorname{supp} K(x, \cdot):=\overline{\left\{y \in \mathbb{R}^{d}: K(x, y) \neq 0\right\}} \subset B_{R}(x)
$$

for all $x \in \mathbb{R}^{d}$. Then it holds:

(i) $A$ is bounded, considered as operator from $L^{\infty}\left(\mathbb{R}^{d}\right)$ to $B C\left(\mathbb{R}^{d}\right)$. 
(ii) $A$ is bounded, considered as operator from $B C_{\infty}\left(\mathbb{R}^{d}\right)$ to $B C_{\infty}\left(\mathbb{R}^{d}\right)$.

Proof. We first note, that the integral defining the operator $A$ is well defined since

$$
\begin{aligned}
\left|\int_{\mathbb{R}^{d}} K(x, y) \psi(y) d y\right| & \leq \int_{\operatorname{supp} K(x, \cdot)}|K(x, y) \| \psi(y)| d y \\
& \leq C \int_{B_{R}(x)} 1 d y\|\psi\|_{L^{\infty}} \\
& \leq C \omega_{d} \int_{0}^{R} r^{d-1} d r\|\psi\|_{L^{\infty}}=C \frac{\omega_{d}}{d} R^{d}\|\psi\|_{L^{\infty}}
\end{aligned}
$$

where we have introduced polar coordinates with origin at $x$ and $\omega_{d}$ denotes the surface area of the unit sphere in $\mathbb{R}^{d}$. As the value does not depend on the point $x \in \mathbb{R}^{d}$ we see that $A \psi \in L^{\infty}\left(\mathbb{R}^{d}\right)$ and $A$ is a bounded operator on $L^{\infty}$ with $\|A\| \leq C \frac{\omega_{d}}{d} R^{d}$.

$K$ is continuous, thus for each $\varepsilon>0$ there exists $\delta=\delta(x, y)>0$ such that

$$
\left|K(x, y)-K\left(x^{\prime}, y\right)\right|<\varepsilon \text { for all } x^{\prime}, y \in \mathbb{R}^{d} \text { with }\left|x-x^{\prime}\right| \leq \delta .
$$

Furthermore using that

$$
\operatorname{supp}\left(K(x, \cdot)-K\left(x^{\prime}, \cdot\right)\right) \subset\left(\operatorname{supp} K(x, \cdot) \cup \operatorname{supp} K\left(x^{\prime}, \cdot\right)\right) \subset\left(B_{R}(x) \cup B_{R}\left(x^{\prime}\right)\right)
$$

we can estimate

$$
\begin{aligned}
\left|(A \psi)(x)-(A \psi)\left(x^{\prime}\right)\right| & \leq \int_{\mathbb{R}^{d}}\left|K(x, y)-K\left(x^{\prime}, y\right)\right||\psi(y)| d y \\
& \leq \varepsilon\|\psi\|_{L^{\infty}} 2 C \int_{B_{R}(x)} 1 d y \\
& \leq \varepsilon\|\psi\|_{L^{\infty}} 2 C \frac{\omega_{d}}{d} R^{d}
\end{aligned}
$$

showing the continuity of $A \psi$ and finishing the proof of (i).

(ii) In the case $\psi \in B C_{\infty}\left(\mathbb{R}^{d}\right)$ we can estimate a little more carefully to obtain

$$
\begin{aligned}
|(A \psi)(x)| & \leq \int_{\operatorname{supp} K(x, \cdot)}|K(x, y)||\psi(y)| d y \\
& \leq \sup _{z \in B_{R}(x)}|\psi(z)| C \int_{B_{R}(x)} 1 d y \\
& \leq \sup _{z \in B_{R}(x)}|\psi(z)| C \frac{\omega_{d}}{d} R^{d} \rightarrow 0, \quad|x| \rightarrow \infty .
\end{aligned}
$$

In the case of a continuous function $\psi$ the essential supremum norm reduces to the usual supremum norm, thus $\|A\|_{B C_{\infty} \rightarrow B C_{\infty}} \leq\|A\|_{L^{\infty} \rightarrow L^{\infty}}$. 
Definition 1.14. A kernel function $K: \mathbb{R}^{d} \times \mathbb{R}^{d} \rightarrow \mathbb{C}$ is local weakly singular, if it is defined and continuous for all $x, y \in \mathbb{R}^{d}, x \neq y$, and there exist positive constants $M$ and $\alpha \in(0, d]$ such that

$$
|K(x, y)| \leq M|x-y|^{\alpha-d}, \quad x, y \in \mathbb{R}^{d}, \quad x \neq y .
$$

LEMMA 1.15. Consider the integral operator

$$
(A \psi)(x):=\int_{\mathbb{R}^{d}} K(x, y) \psi(y) d y, \quad x \in \mathbb{R}^{d}
$$

where $K: \mathbb{R}^{d} \times \mathbb{R}^{d} \rightarrow \mathbb{C}$ denotes a function that is local weakly singular with compact support, i.e. there exists a positive constant $R$ such that $\operatorname{supp} K(x, \cdot) \subset B_{R}(x)$ for all $x \in \mathbb{R}^{d}$. Then it holds:

(i) $A$ is bounded, considered as operator from $L^{\infty}\left(\mathbb{R}^{d}\right)$ to $B C\left(\mathbb{R}^{d}\right)$.

(ii) $A$ is bounded, considered as operator from $B C_{\infty}\left(\mathbb{R}^{d}\right)$ to $B C_{\infty}\left(\mathbb{R}^{d}\right)$.

Proof. We first note that the integral defining the operator $A$ exists as an improper integral in any of the cases $\psi \in B C_{\infty}$ or $\psi \in L^{\infty}$, since the support condition and the local weak singularity yield the following estimate

$$
|K(x, y) \psi(y)| \leq \begin{cases}M\|\psi\|_{L^{\infty}}|x-y|^{\alpha-d}, & y \in \operatorname{supp} K(x, \cdot) \\ 0, & y \notin \operatorname{supp} K(x, \cdot)\end{cases}
$$

and

$$
\int_{\operatorname{supp} K(x, \cdot)}|x-y|^{\alpha-d} d y \leq \int_{B_{R}(x)}|x-y|^{\alpha-d} d y \leq \omega_{d} \int_{0}^{R} r^{\alpha-d} r^{d-1} d r=\frac{\omega_{d}}{\alpha} R^{\alpha},
$$

where we have introduced polar coordinates with origin at $x$ and $\omega_{d}$ denotes the surface area of the unit sphere in $\mathbb{R}^{d}$. As the value does not depend on the point $x \in \mathbb{R}^{d}$ we see that $A \psi \in L^{\infty}\left(\mathbb{R}^{d}\right)$ and $A$ is a bounded operator on $L^{\infty}$ with

$$
\|A\|_{L^{\infty} \rightarrow L^{\infty}} \leq M \frac{\omega_{d}}{\alpha} R^{\alpha}
$$

We continue to prove (i) and show that $A \psi$ is eventually in $B C\left(\mathbb{R}^{d}\right) \subset L^{\infty}\left(\mathbb{R}^{d}\right)$.

For this choose a piecewise linear continuous function $h:[0, \infty) \rightarrow \mathbb{R}$ by setting

$$
h(t):= \begin{cases}0, & 0 \leq t \leq 1 / 2 \\ 2 t-1, & 1 / 2 \leq t \leq 1 \\ 1, & 1 \leq t \leq \infty\end{cases}
$$


and for $n \in \mathbb{N}$ we define the continuous kernel $K_{n}: \mathbb{R}^{d} \times \mathbb{R}^{d} \rightarrow \mathbb{C}$ by

$$
K_{n}(x, y):= \begin{cases}h(n|x-y|) K(x, y), & x \neq y, \\ 0, & x=y .\end{cases}
$$

It follows from LEMMA 1.13 that the corresponding integral operators $A_{n}$ with kernel function $K_{n}$ are bounded operators on $B C\left(\mathbb{R}^{d}\right)$. Furthermore we have the estimate

$$
\begin{aligned}
\left|(A \psi)(x)-\left(A_{n} \psi\right)(x)\right| & =\left|\int_{B_{1 / n}(x)}\{1-h(n|x-y|)\} K(x, y) \psi(y) d y\right| \\
& \leq M\|\psi\|_{L^{\infty} \omega_{d}} \int_{0}^{1 / n} r^{\alpha-d} r^{d-1} d r \\
& =M\|\psi\|_{L^{\infty}} \frac{\omega_{d}}{\alpha n^{\alpha}}, \quad x \in \mathbb{R}^{d} .
\end{aligned}
$$

From this we observe that $A_{n} \psi \rightarrow A \psi, n \rightarrow \infty$, uniformly, and therefore $A \psi \in$ $B C\left(\mathbb{R}^{d}\right)$.

In the case $\psi \in B C_{\infty}\left(\mathbb{R}^{d}\right)$ we can estimate again a little more carefully to obtain

$$
\begin{aligned}
|(A \psi)(x)| & \leq \int_{\operatorname{supp} K(x, \cdot)}|K(x, y)||\psi(y)| d y \\
& \leq M \int_{B_{R}(x)}|x-y|^{\alpha-d}|\psi(y)| d y \\
& \leq \sup _{z \in B_{R}(x)}|\psi(z)| M \int_{B_{R}(x)}|x-y|^{\alpha-d} d y \\
& \leq \sup _{z \in B_{R}(x)}|\psi(z)| M \frac{\omega_{d}}{\alpha} R^{\alpha} \rightarrow 0, \quad|x| \rightarrow \infty .
\end{aligned}
$$

REMARK 1.16. It is clear from the proof that the operator

$$
(|A| \psi)(x):=\int_{\mathbb{R}^{d}}|K(x, y)| \psi(y) d y, \quad x \in \mathbb{R}^{d}
$$

exhibits the same mapping properties as $A$.

\subsubsection{Adjoint operators}

To prove the invertibility of the operator $A$, given through (19), we make use of some well known facts of adjoint operators. We therefore give a short summary of adjoint operators induced by bilinear forms, following [35, Chapter 4]. 
Two normed spaces $X$ and $Y$ equipped with a nondegenerate bilinear form $\langle\cdot, \cdot\rangle$ : $X \times Y \rightarrow \mathbb{C}$ are called a dual system and denoted by $\langle X, Y\rangle$.

We will use a dual system $\left\langle L^{2}(\Gamma), L^{2}(\Gamma)\right\rangle$ where the bilinear form is given through

$$
\langle\varphi, \psi\rangle:=\int_{\Gamma} \varphi(y) \psi(y) d s(y), \quad \varphi, \psi \in L^{2}(\Gamma) .
$$

It is obvious that the integral is well-defined and defines a bilinear form. It remains to show that this form is indeed nondegenerate, i.e. for every $\varphi \in L^{2}(\Gamma)$ with $\varphi \neq 0$ there exists $\psi \in L^{2}(\Gamma)$ such that $\langle\varphi, \psi\rangle \neq 0$. To prove this statement we first note the following connection between this bilinear form and the usual $L^{2}$-scalar product. For $\varphi, \psi \in L^{2}(\Gamma)$ it holds that

$$
\langle\varphi, \psi\rangle=(\varphi, \bar{\psi})
$$

Thus choosing $\psi \equiv \bar{\varphi}$ the proof follows from (1.14) together with the definiteness of the $L^{2}$-scalar product. The bilinear form (1.13)) henceforth is called dual-pairing. In a Hilbert space one allways has a canonical dual-system arising from the inner product.

We can use both dual-systems to define adjoint operators, i.e. two operators $A, A^{\prime}$ : $L^{2}(\Gamma) \rightarrow L^{2}(\Gamma)$ are called adjoint with respect to the dual-pairing if

$$
\langle A \varphi, \psi\rangle=\left\langle\varphi, A^{\prime} \psi\right\rangle \quad \text { for all } \varphi, \psi \in L^{2}(\Gamma)
$$

whereas two operators $A, A^{*}: L^{2}(\Gamma) \rightarrow L^{2}(\Gamma)$ are called adjoint with respect to the canonical dual-system if

$$
(A \varphi, \psi)=\left(\varphi, A^{*} \psi\right) \text { for all } \varphi, \psi \in L^{2}(\Gamma) .
$$

One can establish a connection between these two different adjoint operators through the following calculations. It follows from

$$
\left(\varphi, \overline{A^{\prime} \psi}\right)=\left\langle\varphi, A^{\prime} \psi\right\rangle=\langle A \varphi, \psi\rangle=(A \varphi, \bar{\psi})=\left(\varphi, A^{*} \bar{\psi}\right), \quad \varphi, \psi \in L^{2}(\Gamma)
$$

that $\left(\varphi, A^{*} \bar{\psi}-\overline{A^{\prime} \psi}\right)=0$ for all $\varphi, \psi \in L^{2}(\Gamma)$ which is equivalent to

$$
A^{\prime} \psi=\overline{A^{*} \bar{\psi}} \text { for all } \psi \in L^{2}(\Gamma) .
$$

It is well known that for operators on a Hilbert space $Y$, that are adjoint with respect to the canonical dual-system arising from the inner-product on $Y$, we have that $A$ and $A^{*}$ have the same norm, that $A$ is invertible if and only if $A^{*}$ is invertible, and that if they are both invertible then

$$
\left\|A^{-1}\right\|=\left\|A^{*-1}\right\| .
$$

As the complex conjugation is a norm preserving isomorphism on $L^{2}(\Gamma)$ it follows from (1.15) that all the before mentioned properties immediately transfer to the adjoint operators with respect to the dual-pairing.

A proof for the above statements can be easily constructed from [35, Theorem 4.9, Theorem 15.8]. 


\subsection{Results from differential geometry}

To prove the mapping properties of the double-layer potential operator we need the following Lemma that can be used to prove that in the case of a Lyapunov surface the singularity of the double-layer potential is weakly singular.

LEMma 1.17. In the case of a Lyapunov surface $\Gamma$, i.e. $f \in B C^{1, \alpha}\left(\mathbb{R}^{2}\right)$ it holds that

$$
|\langle\nu(y), x-y\rangle| \leq\|f\|_{B C^{1, \alpha}}|\boldsymbol{x}-\boldsymbol{y}|^{1+\alpha}, \quad x, y \in \Gamma .
$$

Proof. We first note that the inward unit normal at a point $y=(\boldsymbol{y}, f(\boldsymbol{y})) \in \Gamma$ is given through

$$
\nu(y)=\frac{(-\nabla f(\boldsymbol{y}), 1)}{\sqrt{1+|\nabla f(\boldsymbol{y})|^{2}}}=(-\nabla f(\boldsymbol{y}), 1) J(\boldsymbol{y})^{-1} .
$$

Together with the mean value theorem and the lower bound (1.11) on the surface area element $J$ we estimate

$$
\begin{aligned}
|\langle\nu(y), x-y\rangle| & =\frac{1}{\sqrt{1+|\nabla f(\boldsymbol{y})|^{2}}}|\langle\nabla f(\boldsymbol{y}), \boldsymbol{x}-\boldsymbol{y}\rangle-\{f(\boldsymbol{x})-f(\boldsymbol{y})\}| \\
& \leq 1 \cdot|\langle\nabla f(\boldsymbol{y}), \boldsymbol{x}-\boldsymbol{y}\rangle-\langle\nabla f(\boldsymbol{\xi}), \boldsymbol{x}-\boldsymbol{y}\rangle| \\
& \leq|\nabla f(\boldsymbol{y})-\nabla f(\boldsymbol{\xi})||\boldsymbol{x}-\boldsymbol{y}| \\
& \leq\|f\|_{B C^{1, \alpha}}|\boldsymbol{x}-\boldsymbol{y}|^{1+\alpha}
\end{aligned}
$$

where $\boldsymbol{\xi}=\boldsymbol{x}+t(\boldsymbol{y}-\boldsymbol{x}), t \in(0,1)$ denotes some point between $\boldsymbol{x}$ and $\boldsymbol{y}$.

\subsection{Results from scattering theory for bounded obstacles}

To prove the mapping properties of the boundary layer operators over an unbounded domain in Section 2.1 we need the following classical results for operators defined over bounded obstacles $D$ with Lyapunov boundaries.

Definition 1.18 (Lyapunov boundaries). The boundary is said to satisfy a Lyapunov condition if at each point $x \in \Gamma$ the normal vector $\nu$ exists and there are positive constants $l$ and $\alpha$ such that for the angle $\vartheta(x, y)$ between the normal vectors at $x$ and $y$ the estimate

$$
\vartheta(x, y) \leq l|x-y|^{\alpha}, \quad x, y \in \Gamma
$$

holds. 
The following results we cite are taken from [23] and have been proven under the slightly stronger assumption that the boundary is $C^{2}$. Proofs for the case of Lyapunov curves can be found in [29], [43] or [61]. The results however remain the same.

Definition 1.19. Given an integrable function $\varphi$, the integrals

$$
\begin{array}{ll}
u(x):=\int_{\partial D} \Phi(x, y) \varphi(y) d s(y), & x \in \mathbb{R}^{d} \backslash \partial D, \\
v(x):=\int_{\partial D} \frac{\Phi(x, y)}{\partial \nu(y)} \varphi(y) d s(y), \quad x \in \mathbb{R}^{d} \backslash \partial D,
\end{array}
$$

are called, respectively, acoustic single-layer and acoustic double-layer potentials with density $\varphi$.

The normal vector $\nu$ is directed into the exterior of $D$.

TheOrem 1.20. Let $\partial D$ be of class $C^{2}$ and let $\varphi$ be continuous. Then the singlelayer potential $u$ with density $\varphi$ is continuous throughout $\mathbb{R}^{3}$ and

$$
\|u\|_{B C\left(\mathbb{R}^{3}\right)} \leq C\|\varphi\|_{B C(\partial D)}
$$

for some constant $C>0$ depending only on $\partial D$. On the boundary we have

$$
\begin{aligned}
u(x) & =\int_{\partial D} \Phi(x, y) \varphi(y) d s(y), \quad x \in \partial D, \\
\frac{\partial u_{ \pm}}{\partial \nu}(x) & =\int_{\partial D} \frac{\Phi(x, y)}{\partial \nu(y)} \varphi(y) d s(y) \mp \frac{1}{2} \varphi(x), \quad x \in \partial D,
\end{aligned}
$$

where

$$
\frac{\partial u_{ \pm}}{\partial \nu}(x):=\lim _{h \rightarrow 0+}\langle\nu(x), \operatorname{grad} u(x \pm h \nu(x))\rangle
$$

is to be understood in the sense of uniform convergence on $\partial D$ and where the integrals exists as improper integrals. The double-layer potential $v$ with density $\varphi$ can be continuously extended from $D$ to $\bar{D}$ and from $\mathbb{R}^{3} \backslash \bar{D}$ to $\mathbb{R}^{3} \backslash D$ with limiting values

$$
v_{ \pm}(x)=\int_{\partial D} \frac{\Phi(x, y)}{\partial \nu(y)} \varphi(y) d s(y) \pm \frac{1}{2} \varphi(x), \quad x \in \partial D
$$

where

$$
v_{ \pm}(x):=\lim _{h \rightarrow 0+} v(x \pm h \nu(x))
$$

and where the integral exists as an improper integral. Furthermore,

$$
\|v\|_{B C(\bar{D})} \leq C\|\varphi\|_{B C(\partial D)}, \quad\|v\|_{B C\left(\mathbb{R}^{3} \backslash D\right)} \leq C\|\varphi\|_{B C(\partial D)}
$$


for some constant $C>0$ depending only on $\partial D$ and

$$
\lim _{h \rightarrow 0+}\left\{\frac{\partial v}{\partial \nu}(x+h \nu(x))-\frac{\partial v}{\partial \nu}(x-h \nu(x))\right\}
$$

uniformly on $\partial D$.

\subsection{Properties of 3D fundamental solution}

We start with an investigation of properties of the fundamental solution $\Phi(x, y)$ and its derivatives.

The key results are the expansions (1.21) and (1.27) needed to prove mapping properties of the boundary integral operators $S$ and $K$ in Section 2.1.

\subsubsection{Expansion for $G$}

Denote by $U_{c}$ the strip $\overline{D^{-c}} \backslash D^{c} \subset \mathbb{R}^{3}$ for some $c>0$, i.e.

$$
U_{c}=\left\{x \in \mathbb{R}^{3}:-c \leq x_{3} \leq c\right\} .
$$

Then we are interested in understanding the behaviour of $G(x, y)$ and $\Phi(x, y)$ for $x, y \in U_{c}$ with $|x-y| \geq 1$ in terms of $|\boldsymbol{x}-\boldsymbol{y}|$.

We use Taylor expansion for the fundamental solution $\Phi(x, y)$ with respect to variations of $x_{3}$ and $y_{3}$. It follows from Taylor's theorem for $g \in C^{3}\left(\mathbb{R}^{2}\right)$ that

$$
g(\boldsymbol{h})=\sum_{|j| \leq 2} \frac{\partial^{j}}{j !} g(\mathbf{0}) \boldsymbol{h}^{j}+R(\boldsymbol{h}),
$$

where $\boldsymbol{h}=\left(h_{1}, h_{2}\right), j \in \mathbb{N}_{0}^{2}$ denotes a multi-index and $R$ is the remainder term of the expansion. We apply (1.18) to the function

$$
g(\boldsymbol{h}):=\Phi\left(\boldsymbol{x}+h_{1} e_{3}, \boldsymbol{y}+h_{2} e_{3}\right)
$$

where $e_{3}=(0,0,1)$ is the unit vector in the $x_{3}$-direction, $\boldsymbol{y}=\left(y_{1}, y_{2}, 0\right) \in \Gamma^{0}$ and $h_{1}, h_{2} \in[-c, c]$ for some constant $c>0$. We use that

$$
\partial_{x_{3}} \Phi(x, y)=-\partial_{y_{3}} \Phi(x, y), \quad x \neq y,
$$

to express all partial derivatives of $\Phi$ as derivatives with respect to $y_{3}$. Thus

$$
\partial_{x_{3}} \partial_{x_{3}} \Phi=-\partial_{x_{3}} \partial_{y_{3}} \Phi=-\partial_{y_{3}} \partial_{x_{3}} \Phi=\partial_{y_{3}} \partial_{y_{3}} \Phi
$$


and we obtain

$$
\begin{aligned}
& \Phi\left(\boldsymbol{x}+h_{1} e_{3}, \boldsymbol{y}+h_{2} e_{3}\right)=\Phi(\boldsymbol{x}, \boldsymbol{y})-\frac{\partial \Phi(\boldsymbol{x}, \boldsymbol{y})}{\partial y_{3}}\left(h_{1}-h_{2}\right) \\
&+\frac{1}{2} \frac{\partial^{2} \Phi(\boldsymbol{x}, \boldsymbol{y})}{\partial y_{3}^{2}}\left(h_{1}-h_{2}\right)^{2}+R(\boldsymbol{x}, \boldsymbol{y}, \boldsymbol{h}) .
\end{aligned}
$$

For the first two derivatives of $\Phi(x, \cdot)$ with respect to $y_{3}$ we calculate

$$
\frac{\partial \Phi(x, y)}{\partial y_{3}}=-\frac{i \kappa}{4 \pi}\left(x_{3}-y_{3}\right) \frac{e^{i \kappa|x-y|}}{|x-y|^{2}}+\frac{1}{4 \pi}\left(x_{3}-y_{3}\right) \frac{e^{i \kappa|x-y|}}{|x-y|^{3}}
$$

and

$$
\begin{aligned}
\frac{\partial^{2} \Phi(x, y)}{\partial y_{3}^{2}} & =\frac{i \kappa}{4 \pi} \frac{e^{i \kappa|x-y|}}{|x-y|^{2}}-\frac{\kappa^{2}}{4 \pi} \frac{\left(x_{3}-y_{3}\right)^{2}}{|x-y|^{3}} e^{i \kappa|x-y|}-\frac{1}{4 \pi} \frac{e^{i \kappa|x-y|}}{|x-y|^{3}} \\
& -\frac{3 i \kappa}{4 \pi} \frac{\left(x_{3}-y_{3}\right)^{2}}{|x-y|^{4}} e^{i \kappa|x-y|}+\frac{3}{4 \pi} \frac{\left(x_{3}-y_{3}\right)^{2}}{|x-y|^{5}} e^{i \kappa|x-y|}
\end{aligned}
$$

We rewrite the second derivative as

$$
\frac{\partial^{2} \Phi(x, y)}{\partial y_{3}^{2}}-\frac{i \kappa}{4 \pi} \frac{e^{i \kappa|x-y|}}{|x-y|^{2}}=L(x, y, \kappa)
$$

with

$$
\begin{aligned}
L(x, y, \kappa):=- & \frac{\kappa^{2}}{4 \pi} \frac{\left(x_{3}-y_{3}\right)^{2}}{|x-y|^{3}} e^{i \kappa|x-y|}-\frac{1}{4 \pi} \frac{e^{i \kappa|x-y|}}{|x-y|^{3}}-\frac{3 i \kappa}{4 \pi} \frac{\left(x_{3}-y_{3}\right)^{2}}{|x-y|^{4}} e^{i \kappa|x-y|} \\
& +\frac{3}{4 \pi} \frac{\left(x_{3}-y_{3}\right)^{2}}{|x-y|^{5}} e^{i \kappa|x-y|}
\end{aligned}
$$

For $L$ we find the following estimate: for $|x-y| \geq 1$ we use $\left(x_{3}-y_{3}\right) \leq 2 c$ to obtain

$$
\begin{aligned}
|L(x, y, \kappa)| & \leq C_{1} \frac{4 c^{2}}{|x-y|^{3}}+C_{2} \frac{1}{|x-y|^{3}}+C_{3} \frac{4 c^{2}}{|x-y|^{4}}+C_{4} \frac{4 c^{2}}{|x-y|^{5}} \\
& \leq \frac{C}{|x-y|^{3}}
\end{aligned}
$$

for some positive constant $C$ depending only on $c$ and $S \subset \tilde{\mathbb{C}}$.

Thus we see that given $c>0$ and a compact subset $S \subset \tilde{\mathbb{C}}$ there exists a constant $C$, depending only on $c$ and $S$, such that

$$
\left|\frac{\partial^{2} \Phi(x, y)}{\partial y_{3}^{2}}-\frac{i \kappa}{4 \pi} \frac{e^{i \kappa|x-y|}}{|x-y|^{2}}\right| \leq \frac{C}{|x-y|^{3}}
$$


for all $x, y \in U_{c}$ with $|x-y| \geq 1$ and all wavenumbers $\kappa \in S$. We abbreviate this by writing

$$
\frac{\partial^{2} \Phi(x, y)}{\partial y_{3}^{2}}=\frac{i \kappa}{4 \pi} \frac{e^{i \kappa|x-y|}}{|x-y|^{2}}+O\left(\frac{1}{|x-y|^{3}}\right) .
$$

The similar equations below, in particular (1.21) and (1.27), are to be understood in an analogous fashion.

Inserting all of the previously calculated derivatives into (1.19), noting that

$$
\frac{\partial \Phi(\boldsymbol{x}, \boldsymbol{y})}{\partial y_{3}}=0
$$

yields

$$
\begin{aligned}
\Phi\left(\boldsymbol{x}+h_{1} e_{3}, \boldsymbol{y}+h_{2} e_{3}\right) & =\frac{1}{4 \pi} \frac{e^{i \kappa|\boldsymbol{x}-\boldsymbol{y}|}}{|\boldsymbol{x}-\boldsymbol{y}|} \\
& +\frac{1}{4 \pi} \frac{i \kappa e^{i \kappa|\boldsymbol{x}-\boldsymbol{y}|}}{|\boldsymbol{x}-\boldsymbol{y}|^{2}} \frac{\left(h_{1}-h_{2}\right)^{2}}{2}+O\left(\frac{1}{|\boldsymbol{x}-\boldsymbol{y}|^{3}}\right) .
\end{aligned}
$$

Altogether we obtain the following lemma.

LEMMA 1.21. Given $c>0$ and a compact subset $S \subset \tilde{\mathbb{C}}$, there exists a constant $C>0$ such that

$$
\left|G\left(\boldsymbol{x}+h_{1} e_{3}, \boldsymbol{y}+h_{2} e_{3}\right)+\frac{h_{1} h_{2}}{2 \pi} \frac{i \kappa e^{i \kappa|\boldsymbol{x}-\boldsymbol{y}|}}{|\boldsymbol{x}-\boldsymbol{y}|^{2}}\right| \leq \frac{C}{|\boldsymbol{x}-\boldsymbol{y}|^{3}},
$$

for all $\boldsymbol{x}, \boldsymbol{y} \in \mathbb{R}^{2}$ with $|\boldsymbol{x}-\boldsymbol{y}| \geq 1$, all $\kappa \in S$, and all $h_{1}, h_{2} \in[-c, c]$, which we can state again in the more convenient way as

$$
G\left(\boldsymbol{x}+h_{1} e_{3}, \boldsymbol{y}+h_{2} e_{3}\right)=-\frac{1}{2 \pi} \frac{i \kappa e^{i \kappa|\boldsymbol{x}-\boldsymbol{y}|}}{|\boldsymbol{x}-\boldsymbol{y}|^{2}} h_{1} h_{2}+O\left(\frac{1}{|\boldsymbol{x}-\boldsymbol{y}|^{3}}\right) .
$$

Proof. Use (1.20) on

$$
G\left(\boldsymbol{x}+h_{1} e_{3}, \boldsymbol{y}+h_{2} e_{3}\right)=\Phi\left(\boldsymbol{x}+h_{1} e_{3}, \boldsymbol{y}+h_{2} e_{3}\right)-\Phi\left(\boldsymbol{x}+h_{1} e_{3}, \boldsymbol{y}-h_{2} e_{3}\right) .
$$

We also need another bound that is valid in all of the half space and not just inside a strip around the surface. Following [16, Lemma 3.1] we transfer the proof to the three dimensional case and show:

LEMmA 1.22. Given a compact subset $S \subset \tilde{\mathbb{C}}$ there exists a constant $C>0$ depending only on $S$ such that

$$
|G(x, y)| \leq C \frac{\left(1+x_{3}\right)\left(1+y_{3}\right)}{|x-y|^{2}}
$$

for all $x, y \in D^{0}=\left\{x \in \mathbb{R}^{3}: x_{3}>0\right\}$ with $|x-y|>1$, and all $\kappa \in S$. 
Proof. For $x, y \in D^{0}, x \neq y$ with $|x-y|>1$ define $r:=|x-y|, r^{\prime}:=\left|x-y^{\prime}\right|$ and

$$
\phi(s):=\frac{e^{i \kappa s}}{4 \pi s}, \quad s>0 .
$$

From

$$
\phi^{\prime}(s)=i \kappa \frac{e^{i \kappa s}}{4 \pi s}-\frac{e^{i \kappa s}}{4 \pi s^{2}}
$$

we estimate, in the case $s>1$,

$$
\begin{aligned}
\left|\phi^{\prime}(s)\right| & \leq\left|i \kappa \frac{e^{i \kappa_{r} s} e^{-\kappa_{i} s}}{4 \pi s}-\frac{e^{i \kappa_{r} s} e^{-\kappa_{i} s}}{4 \pi s^{2}}\right| \leq|\kappa| \frac{e^{-\kappa_{i} s}}{4 \pi s}+\frac{e^{-\kappa_{i} s}}{4 \pi s^{2}} \\
& \leq|\kappa| \frac{e^{-\kappa_{i} s}}{4 \pi s}+\frac{e^{-\kappa_{i} s}}{4 \pi s} \leq \max (|\kappa|, 1) \frac{e^{-\kappa_{i} s}}{4 \pi s} \leq \frac{C^{\prime}}{s}
\end{aligned}
$$

with a constant $C^{\prime}>0$ depending only on $\kappa$. We note that for $x, y \in D^{0}$ it holds that $r^{\prime}>r$ and furthermore

$$
\left|r^{\prime}-r\right|=\frac{\left(r^{\prime}-r\right)\left(r^{\prime}+r\right)}{r^{\prime}+r}=\frac{r^{\prime 2}-r^{2}}{r^{\prime}+r}=\frac{4 x_{3} y_{3}}{r^{\prime}+r} \leq \frac{4 x_{3} y_{3}}{2 r} \leq \frac{2\left(1+x_{3}\right)\left(1+y_{3}\right)}{r} .
$$

Thus using the mean value theorem we have

$$
\begin{aligned}
|G(x, y)| & =\left|\phi\left(r^{\prime}\right)-\phi(r)\right| \leq\left|r^{\prime}-r\right| \max _{r \leq s \leq r^{\prime}}\left|\phi^{\prime}(s)\right| \\
& \leq\left|r^{\prime}-r\right| \max _{r \leq s \leq r^{\prime}} \frac{C^{\prime}}{s} \leq 2 C^{\prime} \frac{\left(1+x_{3}\right)\left(1+y_{3}\right)}{r^{2}} .
\end{aligned}
$$

This proves the Lemma with $C:=2 C^{\prime}$.

REMARK 1.23. Examining the proof we see that for all $x, y \in D^{0}=\left\{x \in \mathbb{R}^{3}: x_{3}>\right.$ $0\}$ with $|x-y|>1$, and all $\kappa \in \tilde{\mathbb{C}}$ it holds that

$$
|G(x, y)| \leq \frac{\max (|\kappa|, 1)}{2 \pi} \frac{\left(1+x_{3}\right)\left(1+y_{3}\right)}{|x-y|^{2}} e^{-\kappa_{i}|x-y|}
$$

\subsubsection{Expansion for $\partial G / \partial \nu$}

We repeat the above analysis for the normal derivative of $G$. We introduce the linear operator $T: \mathbb{R}^{3} \rightarrow \mathbb{R}^{3}$ given through $T y=y^{\prime}=\left(y_{1}, y_{2},-y_{3}\right)$. Note that this operator commutes with any linear operation on $\mathbb{R}^{3}$. The following abbreviations will be used throughout this work.

$$
\nabla_{y} \Phi(x, y):=(\nabla \Phi(x, \cdot))(y) \quad \text { and } \quad \nabla_{x} \Phi(x, y):=(\nabla \Phi(\cdot, y))(x) .
$$


Straightforward calculations yield

$$
\frac{\partial \Phi(x, y)}{\partial \nu(y)}=\left\langle\nu(y), \nabla_{y} \Phi(x, y)\right\rangle=\frac{1}{4 \pi} \frac{\langle\nu(y), x-y\rangle}{|x-y|^{2}}\left\{\frac{1}{|x-y|}-i k\right\} e^{i k|x-y|} .
$$

Using the chain rule we compute

$$
\nabla_{y}\left\{\Phi\left(x, y^{\prime}\right)\right\}=(\nabla \Phi(x, \cdot))\left(y^{\prime}\right) \circ T
$$

to see that

$$
\begin{aligned}
\frac{\partial \Phi\left(x, y^{\prime}\right)}{\partial \nu(y)} & =\left\langle\nu(y), \nabla_{y}\left\{\Phi\left(x, y^{\prime}\right)\right\}\right\rangle \\
& =\frac{1}{4 \pi} \frac{\left\langle T \nu(y), x-y^{\prime}\right\rangle}{\left|x-y^{\prime}\right|^{2}}\left\{\frac{1}{\left|x-y^{\prime}\right|}-i k\right\} e^{i k\left|x-y^{\prime}\right|} \\
& =\frac{1}{4 \pi} \frac{\left\langle\nu(y)^{\prime}, x-y^{\prime}\right\rangle}{\left|x-y^{\prime}\right|^{2}}\left\{\frac{1}{\left|x-y^{\prime}\right|}-i k\right\} e^{i k\left|x-y^{\prime}\right|}
\end{aligned}
$$

where $\nu(y)^{\prime}=\left(\nu_{1}(y), \nu_{2}(y),-\nu_{3}(y)\right)$. Separating (1.23) and (1.24) into components and introducing the notation $\boldsymbol{\nu}(y):=\left(\nu_{1}(y), \nu_{2}(y)\right)$, we derive

$$
\begin{aligned}
4 \pi \frac{\partial G(x, y)}{\partial \nu(y)} & =-i \kappa \boldsymbol{\nu}(y) \cdot(\boldsymbol{x}-\boldsymbol{y})\left\{\frac{e^{i \kappa|x-y|}}{|x-y|^{2}}-\frac{e^{i \kappa\left|x-y^{\prime}\right|}}{\left|x-y^{\prime}\right|^{2}}\right\} \\
& -\quad i \kappa \nu_{3}(y)\left(x_{3}-y_{3}\right) \frac{e^{i \kappa|x-y|}}{|x-y|^{2}}-i \kappa \nu_{3}(y)\left(x_{3}+y_{3}\right) \frac{e^{i \kappa\left|x-y^{\prime}\right|}}{\left|x-y^{\prime}\right|^{2}} \\
& +\boldsymbol{\nu}(y) \cdot(\boldsymbol{x}-\boldsymbol{y})\left\{\frac{e^{i \kappa|x-y|}}{|x-y|^{3}}-\frac{e^{i \kappa\left|x-y^{\prime}\right|}}{\left|x-y^{\prime}\right|^{3}}\right\} \\
& +\nu_{3}(y)\left(x_{3}-y_{3}\right) \frac{e^{i \kappa|x-y|}}{|x-y|^{3}}+\nu_{3}(y)\left(x_{3}+y_{3}\right) \frac{e^{i \kappa\left|x-y^{\prime}\right|}}{\left|x-y^{\prime}\right|^{3}} .
\end{aligned}
$$

We proceed as in (1.20) and calculate for

$$
\Psi(x, y):=\frac{e^{i \kappa|x-y|}}{|x-y|^{2}}, \quad x \neq y
$$

the expansion

$$
\Psi\left(\boldsymbol{x}+h_{1} e_{3}, \boldsymbol{y}+h_{2} e_{3}\right)=\frac{e^{i \kappa|\boldsymbol{x}-\boldsymbol{y}|}}{|\boldsymbol{x}-\boldsymbol{y}|^{2}}+\frac{i \kappa e^{i \kappa|\boldsymbol{x}-\boldsymbol{y}|}}{|\boldsymbol{x}-\boldsymbol{y}|^{3}} \frac{\left(h_{1}-h_{2}\right)^{2}}{2}+O\left(\frac{1}{|\boldsymbol{x}-\boldsymbol{y}|^{4}}\right)
$$

Inserting this into (1.25) yields 
LEMMA 1.24. Given a compact subset $S \subset \tilde{\mathbb{C}}$ there exists a constant $C>0$ depending only on $S$ such that

$$
\left|\frac{\partial G\left(\boldsymbol{x}+h_{1} e_{3}, \boldsymbol{y}+h_{2} e_{3}\right)}{\partial \nu(y)}+\left(\frac{\kappa^{2} h_{1} h_{2}}{2 \pi}\left\langle\boldsymbol{\nu}(y), \frac{(\boldsymbol{x}-\boldsymbol{y})}{|\boldsymbol{x}-\boldsymbol{y}|}\right\rangle+\frac{i \kappa h_{1}}{2 \pi} \nu_{3}(y)\right) \Psi(\boldsymbol{x}, \boldsymbol{y})\right| \leq \frac{C}{|\boldsymbol{x}-\boldsymbol{y}|^{3}}
$$

for all $\boldsymbol{x}, \boldsymbol{y} \in \mathbb{R}^{2}$ with $|\boldsymbol{x}-\boldsymbol{y}| \geq 1$, all $\kappa \in S$, and all $h_{1}, h_{2} \in[-c, c]$, which we can state again in the more convenient way as

$$
\begin{aligned}
\frac{\partial G\left(\boldsymbol{x}+h_{1} e_{3}, \boldsymbol{y}+h_{2} e_{3}\right)}{\partial \nu(y)} & =-\frac{\kappa^{2}}{2 \pi} \boldsymbol{\nu}(y) \cdot \frac{(\boldsymbol{x}-\boldsymbol{y})}{|\boldsymbol{x}-\boldsymbol{y}|} \frac{e^{i \kappa|\boldsymbol{x}-\boldsymbol{y}|}}{|\boldsymbol{x}-\boldsymbol{y}|^{2}} h_{1} h_{2}- \\
& -\frac{i \kappa}{2 \pi} \nu_{3}(y) \frac{e^{i \kappa|\boldsymbol{x}-\boldsymbol{y}|}}{|\boldsymbol{x}-\boldsymbol{y}|^{2}} h_{1}+O\left(\frac{1}{|\boldsymbol{x}-\boldsymbol{y}|^{3}}\right) .
\end{aligned}
$$

In complete analogy to LEMMA 1.22 one proves:

LEMMA 1.25. Given a compact subset $S \subset \tilde{\mathbb{C}}$ there exists a constant $C>0$ depending only on $S$ such that

$$
\left|\nabla_{y} G(x, y)\right| \leq C \frac{\left(1+x_{3}\right)\left(1+y_{3}\right)}{|x-y|^{2}}
$$

for all $x, y \in D^{0}=\left\{x \in \mathbb{R}^{3}: x_{3}>0\right\}$ with $|x-y|>1$, and all $\kappa \in S$.

\subsection{Results from Fourier analysis}

To establish that $S$ and $K$ are bounded operators on $L^{2}(\Gamma), X(\Gamma)$ and on $X_{\infty}(\Gamma)$ we use Fourier methods together with certain mapping properties of convolution operators. To make this thesis self contained a review of the relevant theorems on Fourier transformation is included. All of the results can be found in more detail e.g. [49], [60], [58] or [53].

\subsubsection{Fourier transformation revisited}

For reasonable functions $\varphi: \mathbb{R}^{d} \rightarrow \mathbb{C}$ the Fourier transform and the inverse Fourier transform are given through the following two linear mappings

$$
(\mathcal{F} \varphi)(k):=\frac{1}{(2 \pi)^{d / 2}} \int_{\mathbb{R}^{d}} e^{-i k \cdot x} \varphi(x) d x, \quad k \in \mathbb{R}^{d}
$$

and

$$
\left(\mathcal{F}^{-1} \varphi\right)(k):=\frac{1}{(2 \pi)^{d / 2}} \int_{\mathbb{R}^{d}} e^{i k \cdot x} \varphi(x) d x, \quad k \in \mathbb{R}^{d}
$$


Classes of reasonable functions e.g. turn out to be $\mathcal{S}\left(\mathbb{R}^{d}\right)$, the Schwartz space of rapidly decreasing functions and the spaces of Lebesgue integrable or squareintegrable functions, $L^{1}\left(\mathbb{R}^{d}\right)$ and $L^{2}\left(\mathbb{R}^{d}\right)$. It is possible to extend the Fourier transformation to the space of generalised functions or tempered distributions $\mathcal{S}^{\prime}\left(\mathbb{R}^{d}\right)$, but this extension is not used in this thesis, though a reader more familiar with distribution theory might find easier arguments to compute the 2D-Fourier transform of the trace of the Dirichlet Green's function on some flat surface $\Gamma_{h}, h>0$, cf. (1.38).

The most rewarding setting for the Fourier transformation is probably $\mathcal{S}$ and $L^{2}$, as the Fourier transform is bijective on these two spaces. In the case of $L^{2}$ it is also a unitary isomorphism, which makes a very elegant theory. The short outline on Fourier analysis we give mostly follows [49].

The following theorems justifies the name inverse Fourier transform as well as the symbol $\mathcal{F}^{-1}$ for the operator given through equation (1.30).

THEOREM 1.26 (Fourier inversion theorem). The Fourier transform is a linear bijective mapping from $\mathcal{S}\left(\mathbb{R}^{d}\right)$ onto $\mathcal{S}\left(\mathbb{R}^{d}\right)$. For $\varphi \in S\left(\mathbb{R}^{d}\right)$ it holds that $\mathcal{F} \mathcal{F}^{-1} \varphi=$ $\mathcal{F}^{-1} \mathcal{F} \varphi=\varphi$ where $\mathcal{F}$ and $\mathcal{F}^{-1}$ are given through (1.29) and (1.30).

Proof. For a proof see [58, Satz 11.5].

The next theorem, known by the name of Plancherel's theorem, shows that Fourier transformation preserves the $L^{2}$-norm as well as the $L^{2}$-inner product.

Theorem 1.27 (Plancherel's Theorem). For $\varphi, \psi \in \mathcal{S}\left(\mathbb{R}^{d}\right)$ it holds

$$
(\mathcal{F} \varphi, \mathcal{F} \psi)_{L^{2}}=(\varphi, \psi)_{L^{2}}
$$

and

$$
\|\mathcal{F} \varphi\|_{L^{2}}=\|\varphi\|_{L^{2}}
$$

Proof. For a proof see [58, Satz 11.9].

Combining the previous two theorems together with the linear extension theorem (THEOREM 1.1) we conclude that there exists a unique continuation to an unitary operator for all of $L^{2}\left(\mathbb{R}^{d}\right)$. This extension is often called Fourier-Plancherel transform.

THEOREM 1.28 (Fourier-Plancherel theorem). The Fourier transform $\mathcal{F}$ extends uniquely to a unitary map $\mathcal{F}_{2}: L^{2}\left(\mathbb{R}^{d}\right) \rightarrow L^{2}\left(\mathbb{R}^{d}\right)$. The inverse transform $\mathcal{F}^{-1}$ extends uniquely to an operator $\mathcal{F}_{2}^{-1}$ that is the Hilbert space adjoint of $\mathcal{F}_{2}$.

The Fourier transformation can be extended also to the space of Lebesgue integrable functions; this is the statement of the following theorem. 
Lemma 1.29 (Riemann-Lebesgue lemma). The operators $\mathcal{F}$ and $\mathcal{F}^{-1}$ can be extended in a unique way to bounded linear operators $\mathcal{F}_{1}$ and $\mathcal{F}_{1}^{-1}$ from $L^{1}\left(\mathbb{R}^{d}\right)$ into $L^{\infty}\left(\mathbb{R}^{d}\right)$ with

$$
\left\|\mathcal{F}_{1}\right\|_{L^{1} \rightarrow L^{\infty}}=\left\|\mathcal{F}_{1}^{-1}\right\|_{L^{1} \rightarrow L^{\infty}} \leq(2 \pi)^{-d / 2} .
$$

Furthermore $\mathcal{F}_{1}\left(L^{1}\left(\mathbb{R}^{d}\right)\right)$ and $\mathcal{F}_{1}^{-1}\left(L^{1}\left(\mathbb{R}^{d}\right)\right)$ are subsets of $C_{\infty}\left(\mathbb{R}^{d}\right)$, i.e. $\mathcal{F}_{1} \varphi$ and $\mathcal{F}_{1}^{-1} \varphi$ are continuous and $\lim _{|x| \rightarrow \infty}\left(\mathcal{F}_{1} \varphi\right)(x)=0$ and $\lim _{|x| \rightarrow \infty}\left(\mathcal{F}_{1}^{-1} \varphi\right)(x)=0$ for all $\varphi \in L^{1}\left(\mathbb{R}^{d}\right)$.

REMARK 1.30. Note the different meaning of the symbol $\mathcal{F}_{1}^{-1}$ in contrast to $\mathcal{F}_{2}^{-1}$. As the range of $\mathcal{F}_{1}$ is not contained in $L^{1}\left(\mathbb{R}^{d}\right)$, the symbol $\mathcal{F}_{1}^{-1}$ does not denote the operator inverse of $\mathcal{F}_{1}$. But as one can show that the Fourier transform on $L^{1}$ is injective, one gets a pointwise inversion formula $\mathcal{F}_{1} \mathcal{F}_{1}^{-1} \psi=\mathcal{F}_{1}^{-1} \mathcal{F}_{1} \psi=\psi$ if one assumes that for $\psi \in L^{1}$ also $\mathcal{F}_{1} \psi \in L^{1}$.

For practical computations it is useful to know that the abstract extensions that are given through the above theorems have a representation as integral operators.

In the case of $L^{1}$-functions the extensions are given through the integral representations (1.29) and (1.30), as can be concluded from the Lebesgue dominated convergence theorem. In the case of $L^{2}$-functions the integrals (1.29) and (1.30) have to be interpreted a little differently as the following example shows.

EXAMPLE 1.31. Consider the piecewise defined function

$$
\varphi(x):= \begin{cases}1, & |x| \leq 1 \\ \frac{1}{|x|}, & |x|>1\end{cases}
$$

which is clearly in $L^{2}(\mathbb{R}) \cap C(\mathbb{R})$ but not in $L^{1}(\mathbb{R}) \cap C(\mathbb{R})$. Thus for $k=0(1.29)$ and (1.30) neither exists as proper nor improper integrals.

In general, if $\varphi \in L^{2}\left(\mathbb{R}^{d}\right)$ the Fourier transform is given as $L^{2}$-limit of a sequence $\left\{\varphi_{R}\right\}$, where $\left\{\varphi_{R}\right\}$ can be any sequence in $L^{1}\left(\mathbb{R}^{d}\right) \cap L^{2}\left(\mathbb{R}^{d}\right)$ converging to $\varphi$ in the $L^{2}$-norm. A very convenient choice for an approximating sequence is to choose

$$
\varphi_{R}(x):= \begin{cases}\varphi(x), & |x| \leq R, \\ 0, & |x|>R .\end{cases}
$$

$\varphi_{R} \in L^{1}\left(\mathbb{R}^{d}\right) \cap L^{2}\left(\mathbb{R}^{d}\right)$ for every $R>0$ and $\left\|\varphi_{R}-\varphi\right\|_{L^{2}} \rightarrow 0, R \rightarrow \infty$ so one can use the integral representation for $L^{1}$-functions to have a meaningful interpretation in the case of $L^{2}$-functions. This is summarised in the next theorem.

THEOREM 1.32 . 
(i) For $\varphi \in L^{1}\left(\mathbb{R}^{d}\right) \cap L^{2}\left(\mathbb{R}^{d}\right)$ it holds that

$$
\mathcal{F}_{1} \varphi=\mathcal{F}_{2} \varphi, \text { a.e. in } \mathbb{R}^{d}
$$

(ii) Let $\varphi \in L^{2}\left(\mathbb{R}^{d}\right)$. Then the following identities hold in $L^{2}$-sense

$$
\begin{aligned}
\left(\mathcal{F}_{2} \varphi\right)(k) & =\lim _{R \rightarrow \infty} \frac{1}{(2 \pi)^{d / 2}} \int_{|x| \leq R} e^{-i k \cdot x} \varphi(x) d x, \quad \text { a.e. in } \mathbb{R}^{d}, \\
\left(\mathcal{F}_{2}^{-1} \varphi\right)(k) & =\lim _{R \rightarrow \infty} \frac{1}{(2 \pi)^{d / 2}} \int_{|x| \leq R} e^{i k \cdot x} \varphi(x) d x, \quad \text { a.e. in } \mathbb{R}^{d} .
\end{aligned}
$$

The integral exists in the sense of Cauchy principle value integral a.e. and 1. i.m. stands for limit in mean, i.e. convergence in the $L^{2}$-norm.

Proof. For a proof see e.g. [58, Satz 11.10].

\subsubsection{Symmetry properties of the Fourier transformation: Hankel transforms}

For later use we need to compute Fourier transforms of functions with certain symmetry properties. The computation can be simplified if one understands how Fourier transformation respects these symmetries. The following results are summarised from [53, Chapter IV, §1].

It is an interesting result of Fourier theory that one can find an orthogonal direct sum decomposition

$$
L^{2}\left(\mathbb{R}^{2}\right)=\bigoplus_{n=-\infty}^{\infty} \mathfrak{H}^{n}
$$

where

$$
\mathfrak{H}^{n}:=\left\{g \in L^{2}\left(\mathbb{R}^{2}\right): \begin{array}{l}
g(\boldsymbol{x})=g_{\mathrm{rad}}(r) e^{i n \theta} \text { a.e. for some measurable } \\
\text { function satisfying } \int_{0}^{\infty}\left|g_{\mathrm{rad}}(r)\right|^{2} r d r<\infty
\end{array}\right\}, \quad n \in \mathbb{Z},
$$

are subspaces that are invariant with respect to Fourier transformation. This decomposition is related to the fact that Fourier transformation commutes with the action of rotations. Though this decomposition can be derived for arbitrary space dimensions we restrict our presentation to the two-dimensional case that is needed for the application we have in mind.

We now show that the 2D-Fourier transformation on these subspaces reduces to a one dimensional integral that is associated with the so called Hankel transform. Assume first that $g \in L^{1}\left(\mathbb{R}^{2}\right) \cap \mathfrak{H}^{n}$ for some $n \in \mathbb{Z}$. Then it follows from our remarks in Section 1.1.2 on radial functions that $g_{\mathrm{rad}} \in L^{1}\left(\mathbb{R}_{\geq 0}, r d r\right)$. Using polar coordinates 
$(R, \varphi)$ for $\boldsymbol{x}$ and $(r, \theta)$ for $\boldsymbol{y}$ together with the following integral representation of the Bessel functions of the first kind of order $n$

$$
J_{n}(r)=\frac{1}{2 \pi i^{n}} \int_{0}^{2 \pi} e^{i n \theta} e^{i r \cos \theta} d \theta, \quad n \in \mathbb{Z}, r \in \mathbb{R},
$$

and the relations $\boldsymbol{x} \cdot \boldsymbol{y}=R r \cos (\theta-\varphi)$ and $J_{n}(-r)=(-1)^{n} J_{n}(r)$, for $n \in \mathbb{Z}, r \in \mathbb{R}$, we compute that

$$
\begin{aligned}
\left(\mathcal{F}_{1} g\right)(\boldsymbol{x}) & =\frac{1}{2 \pi} \int_{\mathbb{R}^{2}} g(\boldsymbol{y}) e^{-i \boldsymbol{x} \cdot \boldsymbol{y}} d \boldsymbol{y} \\
& =\frac{1}{2 \pi} \int_{0}^{\infty} \int_{0}^{2 \pi} g_{\mathrm{rad}}(r) e^{i n \theta} e^{-i R r \cos (\theta-\varphi)} d \theta r d r \\
& =\frac{1}{2 \pi} \int_{0}^{\infty}\left(\int_{0}^{2 \pi} e^{i n \tilde{\theta}} e^{-i R r \cos \tilde{\theta}} d \tilde{\theta}\right) g_{\mathrm{rad}}(r) r d r e^{i n \varphi} \\
& =i^{n}\left(\int_{0}^{\infty} g_{\mathrm{rad}}(r) J_{n}(-R r) r d r\right) e^{i n \varphi} \\
& =(-i)^{n}\left(\int_{0}^{\infty} g_{\mathrm{rad}}(r) J_{n}(R r) r d r\right) e^{i n \varphi} .
\end{aligned}
$$

It follows from the estimate

$$
\left|\int_{0}^{\infty} g_{\mathrm{rad}}(r) J_{n}(R r) r d r\right| \leq \int_{0}^{\infty}\left|g_{\mathrm{rad}}(r)\right| r d r<\infty
$$

that the integral (1.31) defines an integral operator on $L^{1}\left(\mathbb{R}_{\geq 0}, r d r\right)$ through

$$
\left(\mathcal{H}_{n} \psi\right)(k):=\int_{0}^{\infty} \psi(r) J_{n}(k r) r d r, \quad k \in[0, \infty), n \in \mathbb{N}_{0} .
$$

This transform is sometimes either called the $n$-th order Bochner-Hankel, FourierBessel or Hankel transform - we will use the term Hankel transform. Thus we have the following relation

$$
\left(\mathcal{F}_{1} g\right)(\boldsymbol{x})=(-i)^{n}\left(\mathcal{H}_{n} g_{\mathrm{rad}}\right)(r) e^{i n \theta}
$$

for all $g \in L^{1} \cap \mathfrak{H}^{n}$. In a completely analogue fashion one gets the relation

$$
\left(\mathcal{F}_{1}^{-1} g\right)(\boldsymbol{x})=i^{n}\left(\mathcal{H}_{n} g_{\mathrm{rad}}\right)(r) e^{i n \theta}
$$

which shows that $\mathcal{H}_{n}$ is self-inverse, i.e. $\mathcal{H}_{n}^{2}=I$.

It is possible to extend the domain of the Hankel transform to $L^{2}\left(\mathbb{R}_{\geq 0}, r d r\right)$ in essentially the same way as this was done for the Fourier transformation. Again one has to clarify in what sense the integral representation is to be understood. In the 
case that $g \in \mathfrak{H}^{n}$ we know that $G:=\mathcal{F}_{2} g \in \mathfrak{H}^{n}$, thus there exists a representation $G(\boldsymbol{x})=G_{\mathrm{rad}}(R) e^{i n \varphi}$ a.e. with $G_{\mathrm{rad}} \in L^{2}\left(\mathbb{R}_{\geq 0}, r d r\right)$ and so it follows from (1.31) that

$$
\begin{aligned}
& \int_{\mathbb{R}^{2}}\left|G(\boldsymbol{x})-\frac{1}{2 \pi} \int_{|\boldsymbol{y}| \leq R} g(\boldsymbol{y}) e^{i \boldsymbol{x} \cdot \boldsymbol{y}} d \boldsymbol{y}\right|^{2} d \boldsymbol{x} \\
& =\int_{0}^{\infty} \int_{0}^{2 \pi}\left|G_{\mathrm{rad}}(R) e^{i n \varphi}-(-i)^{n} \int_{0}^{R} g_{\mathrm{rad}}(r) J_{n}(R r) r d r e^{i n \varphi}\right|^{2} d \varphi R d R \\
& =2 \pi \int_{0}^{\infty}\left|G_{\mathrm{rad}}(R)-(-i)^{n} \int_{0}^{R} g_{\mathrm{rad}}(r) J_{n}(R r) r d r\right|^{2} R d R \rightarrow 0, R \rightarrow \infty
\end{aligned}
$$

i.e. $(-i)^{n} \int_{0}^{R} g_{\text {rad }}(r) J_{n}(R r) r d r$ converges to the radial part $G_{\text {rad }}$ of the Fourier transform of $g$ in $L^{2}\left(\mathbb{R}_{\geq 0}, r d r\right)$. Thus the identity (1.32) extends to $g \in \mathfrak{H}^{n}, n \in \mathbb{Z}$, if one understands the integral in the sense of an $L^{2}$-limit of an improper integral. Note that (1.32) can be used to extend the domain of the Fourier transform to an even larger class of functions, e.g. distributions, as the one dimensional integral may exists even if $g_{\text {rad }}$ is not an $L^{1}$ - or $L^{2}$-function.

The preceding analysis allows us to handle the more general symmetries that arise in the expansion of the Green's function, see (1.27). So we use the representation (1.31) to calculate the Fourier transforms of parts of the kernels of the operators $S$ and $K$ that are given in the form

$$
\ell(\boldsymbol{y}):=F(r) e^{i n \theta}:=\frac{e^{i \kappa r}}{\beta+r^{2}} e^{i n \theta}
$$

for some $\beta>0$ and $n=-1,0,1$. Clearly, $\ell \in L^{2}\left(\mathbb{R}^{2}\right)$ for $\beta>0$ and so $\mathcal{F}_{2} \ell$ surely exists. To prove that $S$ and $K$ are bounded operators on $L^{2}(\Gamma)$, cf. LEMma 2.3, we need to know that $\mathcal{F}_{2} \ell \in L^{\infty}\left(\mathbb{R}^{2}\right)$. Thus we show the following lemma.

Lemma 1.33. The Fourier transform of $\ell$ given through (1.33) is bounded.

Proof. To prove the lemma it suffices to show that the improper integral

$$
I(k):=\int_{0}^{\infty} F(r) J_{n}(k r) r d r
$$

is bounded on $[0, \infty)$. With the help of the asymptotic expansion of the Bessel function, cf. for example [1],

$$
J_{n}(z)=\sqrt{\frac{2}{\pi z}} \cos \left(z-\frac{n \pi}{2}-\frac{\pi}{4}\right)\left\{1+O\left(\frac{1}{z}\right)\right\}, \quad|z| \rightarrow \infty,
$$

we see that $e^{i z} J_{n}(z)$ is bounded in $0<\arg z<\theta$, for every $\theta \in(0, \pi / 2)$. Since $\Re(i(\kappa-k) z)=-\left(\kappa_{r}-k\right) \Im(z)-\kappa_{i} \Re(z)$ and $F(z)$ is a holomorphic function in 
$\Re(z)>0$, we see that for $0 \leq k<\kappa_{r}$ we may transform the integral

$$
I(k)=\int_{0}^{\infty} \frac{e^{i(\kappa-k) z}}{\beta+z^{2}} e^{i k z} J_{n}(k z) z d z
$$

into

$$
I(k)=\int_{\gamma} \frac{e^{i(\kappa-k) z}}{\beta+z^{2}} e^{i k z} J_{n}(k z) z d z
$$

with $\gamma=\{(1+i) t: t \geq 0\}$. This integral is bounded for $0<k \leq \kappa_{r} / 2$.

For $k \geq \kappa_{r} / 2$ we can use (1.34) and that $J_{n}(z)$ is continuous and thus, by (1.34), bounded on $[0, \infty)$, to estimate that, for some constants $C_{1}$ and $C_{2}$,

$$
\left|\int_{0}^{\infty} F(r) J_{n}(k r) r d r\right| \leq C_{1}+C_{2} \int_{1}^{\infty} \frac{1}{r^{3 / 2}} d r .
$$

We conclude that $I$ is bounded on $[0, \infty)$, so that, by $(1.32), \mathcal{F}_{2} \ell \in L^{\infty}\left(\mathbb{R}^{2}\right)$.

In order to establish a limiting absorption principle in later sections, we are interested in the dependence of $\ell$ on $\kappa_{i}$.

LEMma 1.34. Denoting $\ell$ by $\ell_{\kappa_{i}}$ to indicate their dependence on the imaginary part of the wavenumber $\kappa_{i}$, we have that $\left\|\mathcal{F}_{2} \ell_{\kappa_{i}}-\mathcal{F}_{2} \ell_{0}\right\|_{L^{\infty}\left(\mathbb{R}^{2}\right)} \rightarrow 0$ as $\kappa_{i} \rightarrow 0$

Proof. From (1.36), since $e^{i z} J_{n}(z)$ is bounded on $\gamma=\{(1+i) t: t \geq 0\}$, we see that, for some constant $C>0$,

$$
\left|I_{\kappa_{i}}(k)-I_{0}(k)\right|<C \int_{0}^{\infty} e^{-\kappa_{r} t / 2}\left(1-e^{-\kappa_{i} t}\right) d t
$$

for $0 \leq k \leq \kappa_{r} / 2, \kappa_{i} \geq 0$, so that $I_{\kappa_{i}}(k) \rightarrow I_{0}(k)$ as $\kappa_{i} \rightarrow 0$, uniformly on $\left[0, \kappa_{r} / 2\right]$. Similarly, using (1.35) and (1.34), cf. (1.37), we can show that $I_{\kappa_{i}}(k) \rightarrow I_{0}(k)$ as $\kappa_{i} \rightarrow 0$, uniformly on $\left[\kappa_{r} / 2, \infty\right]$.

We close this section with yet another example of a Hankel transform. For $h \geq 0$ define

$$
W_{h}(r):=\frac{1}{4 \pi} \frac{e^{i \kappa \sqrt{r^{2}+(2 h)^{2}}}}{\sqrt{r^{2}+(2 h)^{2}}}, \quad r>0 .
$$

We note that this function is surely neither in $L^{1}\left(\mathbb{R}_{\geq 0}, r d r\right)$ nor $L^{2}\left(\mathbb{R}_{\geq 0}, r d r\right)$. However it is possible to show the following lemma.

LEMMA 1.35.

$$
\left(\mathcal{H}_{0} W_{h}\right)(k)=\int_{0}^{\infty} W_{h}(r) J_{0}(k r) r d r=\frac{1}{4 \pi} \frac{e^{-2 h \sqrt{k^{2}-\kappa^{2}}}}{\sqrt{k^{2}-\kappa^{2}}},
$$


for all $k \neq \kappa, h \geq 0$, where the branch of the square root is taken so that its argument lies in $[-\pi / 2,0]$, i.e.

$$
\sqrt{k^{2}-\kappa^{2}} \in V:=\{z \in \mathbb{C}: \Re(z) \geq 0, \Im(z) \leq 0\}
$$

for any wavenumber $\kappa \in \tilde{\mathbb{C}}$ and all $k \geq 0$. Thus explicitly in the case of a real wavenumber this means that

$$
\sqrt{k^{2}-\kappa^{2}}=\left\{\begin{aligned}
-i \sqrt{\kappa^{2}-k^{2}}, & 0 \leq k \leq \kappa \\
\sqrt{k^{2}-\kappa^{2}}, & k \geq \kappa
\end{aligned}\right.
$$

Proof. We write the integral (1.39) in the equivalent form

$$
\frac{1}{4 \pi \sqrt{k}} \int_{0}^{\infty} \sqrt{r} W_{h}(r) J_{0}(k r) \sqrt{r k} d r
$$

and note that

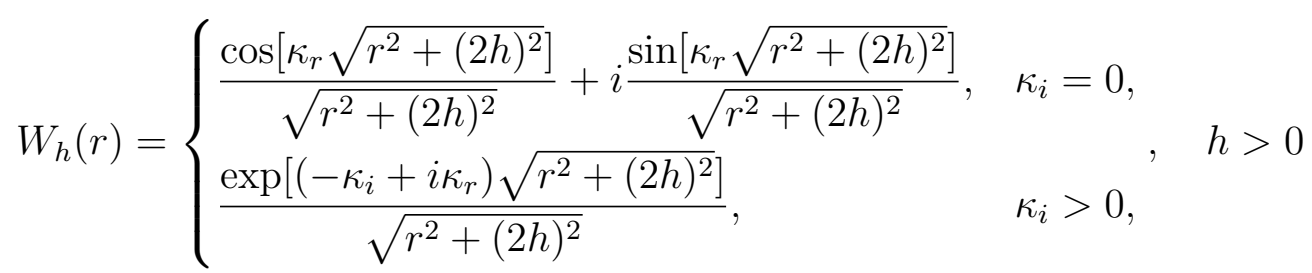

and

$$
W_{0}(r)= \begin{cases}\frac{\cos \left[\kappa_{r} r\right]}{r}+i \frac{\sin \left[\kappa_{r} r\right]}{r}, & \kappa_{i}=0 \\ \frac{\exp \left[\left(-\kappa_{i}+i \kappa_{r}\right) r\right]}{r}, & \kappa_{i}>0\end{cases}
$$

We need to distinguish four cases:

1. $h>0$ and $\kappa_{i}>0$. This follows from [27, Section 8.2, formula (24)]

$$
\int_{0}^{\infty} \sqrt{r} \frac{\exp \left[-\alpha \sqrt{r^{2}+\beta^{2}}\right]}{\sqrt{r^{2}+\beta^{2}}} J_{0}(k r) \sqrt{r k} d r=\sqrt{k} \frac{\exp \left[-\beta \sqrt{k^{2}+\alpha^{2}}\right]}{\sqrt{k^{2}+\alpha^{2}}},
$$

which is valid for $\Re(\alpha), \Re(\beta)>0$. The choice $\alpha=\kappa_{i}-i \kappa_{r}=-i \kappa$ and $\beta=2 h$ clearly satisfies this condition.

2. $h>0$ and $\kappa_{i}=0$. This follows from [27, Section 8.2, formulas (41) and (50)]

$$
\int_{0}^{\infty} \sqrt{r} \frac{\sin \left[\alpha \sqrt{r^{2}+\beta^{2}}\right]}{\sqrt{r^{2}+\beta^{2}}} J_{0}(k r) \sqrt{r k} d r=\left\{\begin{array}{ll}
\sqrt{k} \frac{\cos \left[\beta \sqrt{\alpha^{2}-k^{2}}\right]}{\sqrt{\alpha^{2}-k^{2}}}, & 0<k<\alpha, \\
0, & \alpha<k<\infty,
\end{array},\right.
$$


and

$$
\int_{0}^{\infty} \sqrt{r} \frac{\cos \left[\alpha \sqrt{r^{2}+\beta^{2}}\right]}{\sqrt{r^{2}+\beta^{2}}} J_{0}(k r) \sqrt{r k} d r=\left\{\begin{array}{ll}
-\sqrt{k} \frac{\sin \left[\beta \sqrt{\alpha^{2}-k^{2}}\right]}{\sqrt{\alpha^{2}-k^{2}}}, & 0<k<\alpha, \\
\sqrt{k} \frac{\exp \left[-\beta \sqrt{k^{2}-\alpha^{2}}\right]}{\sqrt{k^{2}-\alpha^{2}}}, & \alpha<k<\infty,
\end{array},\right.
$$

which are both valid for $\alpha, \Re(\beta)>0$. The choice $\alpha=\kappa_{r}$ and $\beta=2 h$ clearly satisfy this condition.

3. $h=0$ and $\kappa_{i}>0$. This follows from [27, Section 8.2, formula (18)]

$$
\int_{0}^{\infty} \frac{\exp [-\alpha r]}{\sqrt{r}} J_{0}(k r) \sqrt{r k} d r=\sqrt{k} \frac{1}{\sqrt{k^{2}+\alpha^{2}}}, \quad \Re(\alpha)>0,
$$

with $\alpha=\kappa_{i}-i \kappa_{r}=-i \kappa$.

4. $h=0$ and $\kappa_{i}=0$. This follows from [27, Section 8.2, formulas (32) and (42)]

$$
\int_{0}^{\infty} \frac{\sin [\alpha r]}{\sqrt{r}} J_{0}(k r) \sqrt{r k} d r= \begin{cases}\sqrt{k} \frac{1}{\sqrt{\alpha^{2}-k^{2}}}, & 0<k<\alpha, \\ 0, & \alpha<k \infty,\end{cases}
$$

and

$$
\int_{0}^{\infty} \frac{\cos [\alpha r]}{\sqrt{r}} J_{0}(k r) \sqrt{r k} d r= \begin{cases}0, & 0<k<\alpha \\ \sqrt{k} \frac{1}{\sqrt{\alpha^{2}-k^{2}}}, & \alpha<k \infty\end{cases}
$$

which are valid for $\alpha>0$. The choice $\alpha=\kappa_{r}$ clearly satisfy this condition.

REMARK 1.36. The integral (1.39) is related to the Sommerfeld integral

$$
\frac{1}{4 \pi} \frac{e^{i \kappa \sqrt{|\boldsymbol{x}|^{2}+x_{3}^{2}}}}{\sqrt{|\boldsymbol{x}|^{2}+x_{3}^{2}}}=\frac{1}{4 \pi} \int_{0}^{\infty} \frac{e^{-\left|x_{3}\right| \sqrt{r^{2}-\kappa^{2}}}}{\sqrt{r^{2}-\kappa^{2}}} J_{0}(r|\boldsymbol{x}|) r d r, \quad x=\left(\boldsymbol{x}, x_{3}\right) \in \mathbb{R}^{3},
$$

where again the branch of the square root with argument in $[-\pi / 2,0]$ has to be used. Using that $\mathcal{H}_{n}$ is self-inverse we get the above identity by formally applying the Hankel transform on both sides of (1.39).

The function $W_{h}$ is important in the sequel as in the case of a flat scattering surface $\Gamma_{h}=\left\{y=(\boldsymbol{y}, h): \boldsymbol{y} \in \mathbb{R}^{2}\right\}$ for some $h>0$, the kernels of the double- and single-layer potential operators can be written in terms of this function. Indeed, for 
points $x=(\boldsymbol{x}, h), y=(\boldsymbol{y}, h) \in \Gamma_{h}$, we find that for the kernel of the single-layer potential operator it holds that

$$
2 G(x, y)=Q_{h}(\boldsymbol{x}-\boldsymbol{y}), \quad x, y \in \Gamma_{h}, x \neq y,
$$

where

$$
Q_{h}(\boldsymbol{y}):=q_{h}(|\boldsymbol{y}|)
$$

and

$$
q_{h}(r):=2\left\{\frac{1}{4 \pi} \frac{e^{i \kappa r}}{r}-\frac{1}{4 \pi} \frac{e^{i \kappa \sqrt{r^{2}+(2 h)^{2}}}}{\sqrt{r^{2}+(2 h)^{2}}}\right\}, \quad r>0
$$

with the obvious equality $q_{h}(r)=2\left\{W_{0}(r)-W_{h}(r)\right\}$. With the help of LEMma 1.35 we can deduce the following theorem.

LEMMA 1.37. In the case of a flat scattering surface $\Gamma_{h}$, for some $h>0$, the Fourier transform of the kernel of the single-layer potential operator is bounded for any choice of a wavenumber $\kappa \in \tilde{\mathbb{C}}$ and is given through

$$
\left(\mathcal{F}_{2} Q_{h}\right)(\boldsymbol{k})=\frac{1}{2 \pi} \frac{1-e^{-2 h \sqrt{|\boldsymbol{k}|^{2}-\kappa^{2}}}}{\sqrt{|\boldsymbol{k}|^{2}-\kappa^{2}}}, \quad \boldsymbol{k} \in \mathbb{R}^{2} .
$$

Proof. The functions $Q_{h} \in L^{2}\left(\mathbb{R}^{2}\right)$, as it follows from our analysis in Section 1.4.1. So the Fourier transform exists and is given through

$$
\begin{aligned}
\left(\mathcal{F}_{2} Q_{h}\right)(\boldsymbol{k}) & =\left(\mathcal{H}_{0} q_{h}\right)(|\boldsymbol{k}|) \\
& =2 \int_{0}^{\infty}\left\{W_{0}(r)-W_{h}(r)\right\} J_{0}(|\boldsymbol{k}| r) r d r \\
& =\frac{1}{2 \pi} \frac{1}{\sqrt{|\boldsymbol{k}|^{2}-\kappa^{2}}}-\frac{1}{2 \pi} \frac{e^{-2 h \sqrt{|\boldsymbol{k}|^{2}-\kappa^{2}}}}{\sqrt{|\boldsymbol{k}|^{2}-\kappa^{2}}} \\
& =\frac{1}{2 \pi} \frac{1-e^{-2 h \sqrt{|\boldsymbol{k}|^{2}-\kappa^{2}}}}{\sqrt{|\boldsymbol{k}|^{2}-\kappa^{2}}},
\end{aligned}
$$

where we used (1.39). We note that the function $g: z \mapsto\left(1-e^{-2 h z}\right) / z$ is analytic, since the singularity at 0 is removable, and it is bounded for all $z=z_{r}+i z_{i} \in V$, with $V$ given through (1.40). In fact $|g(z)| \leq C$ for some constant $C>0$ for all $z \in V$ with $|z| \leq 1$ as $g$ is a continuous function on a compact set. In the case $z \in V$ with $|z|>1$ we estimate

$$
\left|\frac{1-e^{-2 h z}}{z}\right| \leq \frac{1+e^{-2 h z_{r}}}{|z|} \leq 2,
$$

thus proving that $g$ is bounded on $V$. 
To handle the case of the double-layer potential we note that it follows from (1.25) that

$$
2 \frac{\partial G(x, y)}{\partial \nu(y)}=P_{h}(\boldsymbol{x}-\boldsymbol{y}), \quad x, y \in \Gamma_{h}, \quad x \neq y
$$

where

$$
P_{h}(\boldsymbol{y}):=p_{h}(|\boldsymbol{y}|),
$$

and

$$
p_{h}(r):=-\frac{i \kappa h}{\pi} \frac{e^{i \kappa \sqrt{r^{2}+(2 h)^{2}}}}{\left(\sqrt{r^{2}+(2 h)^{2}}\right)^{2}}+\frac{h}{\pi} \frac{e^{i \kappa \sqrt{r^{2}+(2 h)^{2}}}}{\left(\sqrt{r^{2}+(2 h)^{2}}\right)^{3}}, \quad r>0 .
$$

An easy calculation yields $p_{h}(r)=-\frac{\partial}{\partial h}\left\{W_{h}(r)\right\}$. The analogue result to LEMMA 1.37 is again a consequence of (1.39).

LEMMA 1.38. In the case of a flat scattering surface $\Gamma_{h}$, for some $h>0$, the Fourier transform of the kernel of the double-layer potential operator is bounded for any choice of a wavenumber $\kappa \in \tilde{\mathbb{C}}$ and is given through

$$
\left(\mathcal{F}_{2} P_{h}\right)(\boldsymbol{k})=\frac{1}{2 \pi} e^{-2 h \sqrt{|\boldsymbol{k}|^{2}-\kappa^{2}}}, \quad \boldsymbol{k} \in \mathbb{R}^{2} .
$$

Proof. The functions $P_{h} \in L^{2}\left(\mathbb{R}^{2}\right)$, as it follows from our analysis in Section 1.4.2. So the Fourier transform exists and is given through

$$
\begin{aligned}
\left(\mathcal{F}_{2} P_{h}\right)(\boldsymbol{k}) & =\left(\mathcal{H}_{0} p_{h}\right)(|\boldsymbol{k}|) \\
& =-\int_{0}^{\infty} \frac{\partial}{\partial h}\left\{W_{h}(r)\right\} J_{0}(|\boldsymbol{k}| r) r d r \\
& =-\frac{\partial}{\partial h} \int_{0}^{\infty} W_{h}(r) J_{0}(|\boldsymbol{k}| r) r d r \\
& =-\frac{\partial}{\partial h}\left\{\frac{1}{4 \pi} \frac{e^{-2 h \sqrt{|\boldsymbol{k}|^{2}-\kappa^{2}}}}{\sqrt{|\boldsymbol{k}|^{2}-\kappa^{2}}}\right\}=\frac{1}{2 \pi} e^{-2 h \sqrt{|\boldsymbol{k}|^{2}-\kappa^{2}}} .
\end{aligned}
$$

The interchange of integration and differentiation with respect to $h$ is certainly justified whenever $k>0$ and $k \neq \kappa$. For then the integral (1.39) is well-defined and, using the asymptotic estimate of the Bessel function (1.34), we see that for every $H>0$ there exists a constant $C>0$ such that

$$
\left|\frac{\partial W_{h}(r)}{\partial h} J_{0}(k r) r\right|=\left|\frac{\partial}{\partial h}\left\{\frac{1}{4 \pi} \frac{e^{i \kappa \sqrt{r^{2}+(2 h)^{2}}}}{\sqrt{r^{2}+(2 h)^{2}}}\right\} J_{0}(k r) r\right| \leq \frac{C}{r^{3 / 2}},
$$

for $r \geq 1$ and $0 \leq h \leq H$. The boundedness of the function $g: z \mapsto e^{-2 h z}$ on $V$ is obvious. 


\subsection{Convolution}

In the case that the scattering surface $\Gamma$ is a flat plane the integral operators are convolution operators, i.e. the kernel function depends only on the distance of the evaluation and integration points. In this section we study some of their mapping properties and explain their interaction with Fourier transformation.

The presentation mostly follows [39] and [58].

Definition 1.39. For measurable functions $f, g: \mathbb{R}^{d} \rightarrow \mathbb{K}$ the convolution is given through

$$
(f * g)(x):=\int_{\mathbb{R}^{d}} f(x-y) g(y) d y, \quad x \in \mathbb{R}^{d}
$$

whenever the integrand belongs to $L^{1}\left(\mathbb{R}^{d}\right)$.

The substitution $y=x-z$ shows that $(f * g)$ exists if and only if $(g * f)$ exists. Thus convolution is commutative.

The next theorem gives simple criteria for the existence of the convolution.

THEOREM 1.40. Let $1 \leq p, q, s \leq \infty$ and suppose that $\frac{1}{p}+\frac{1}{q}=1+\frac{1}{s}$ with the usual convention $\frac{1}{\infty}=0$.

(i) If $f \in L^{p}\left(\mathbb{R}^{d}\right), g \in L^{q}\left(\mathbb{R}^{d}\right)$, then $(f * g)(x)$ exists a.e. and belongs to $L^{s}\left(\mathbb{R}^{d}\right)$ with

$$
\|f * g\|_{L^{s}} \leq\|f\|_{L^{p}}\|g\|_{L^{q}} .
$$

(ii) If $s=\infty$ it holds moreover that $f * g$ is uniformly continuous on $\mathbb{R}^{d}$.

(iii) If $s=\infty$ and $1<p, q<\infty$ then $\lim _{|x| \rightarrow \infty}(f * g)(x)=0$, i.e. $f * g \in B C_{\infty}\left(\mathbb{R}^{d}\right)$.

Proof. For a detailed proof see [39, Theorem 3.1, Theorem 3.2].

REMARK 1.41. The inequality (1.45) is referred to as Young's inequality. The statement of (iii) can be slightly improved to the case $p=1$ and $q=\infty$ if one uses the closed subspace $B C_{\infty}\left(\mathbb{R}^{d}\right) \subset L^{\infty}\left(\mathbb{R}^{d}\right)$, i.e. for $f \in L^{1}\left(\mathbb{R}^{d}\right)$ and $g \in B C_{\infty}\left(\mathbb{R}^{d}\right)$ (or vice versa) it holds that $f * g \in B C_{\infty}\left(\mathbb{R}^{d}\right)$. For a proof of this statement for the case of one dimension see [11, Proposition 0.2.1]. The multi-dimensional cases can be handled in a similar way.

The following convolution theorem is the key to derive an analytical solution of convolution integral equations. It basically states that the convolution product transforms to a pointwise multiplication in Fourier space and therefore yields a rather simple algebraic equation that can be solved and afterwards transformed back.

The easiest setting to formulate the theorem is provided by the class of Schwartz functions. 
TheOREM 1.42 (Convolution theorem on $\mathcal{S}$ ). For $f, g \in \mathcal{S}\left(\mathbb{R}^{d}\right)$ the Convolution theorem holds

$$
\mathcal{F}(f * g)=(2 \pi)^{d / 2}(\mathcal{F} f)(\mathcal{F} g) .
$$

Proof. For a proof see [49, Theorem IX.3].

There also is an analogue formulation of the Convolution theorem for $L^{2}$ functions.

Theorem 1.43 (Convolution theorem for $L^{2}$ ).

(i) For $f, g \in L^{2}\left(\mathbb{R}^{d}\right)$ the convolution formulae can be written as

$$
f * g=(2 \pi)^{d / 2} \mathcal{F}_{1}^{-1}\left(\mathcal{F}_{2} f \cdot \mathcal{F}_{2} g\right) .
$$

(ii) For $f, g \in L^{2}\left(\mathbb{R}^{d}\right)$ the following statements are equivalent:

(a) $\mathcal{F}_{2} f \cdot \mathcal{F}_{2} g \in L^{2}\left(\mathbb{R}^{d}\right)$,

(b) $\mathcal{F}_{2}^{-1} f \cdot \mathcal{F}_{2}^{-1} g \in L^{2}\left(\mathbb{R}^{d}\right)$,

(c) $f * g \in L^{2}\left(\mathbb{R}^{d}\right)$.

In this case the convolution formulae can be written as

$$
\mathcal{F}_{2}(f * g)=(2 \pi)^{d / 2} \mathcal{F}_{2} f \cdot \mathcal{F}_{2} g \quad \Leftrightarrow \quad f * g=(2 \pi)^{d / 2} \mathcal{F}_{2}^{-1}\left(\mathcal{F}_{2} f \cdot \mathcal{F}_{2} g\right) .
$$

Proof. For a proof see [58, Satz 11.11].

\subsubsection{Convolution operators}

We use the results from the previous sections to prove mapping properties of certain convolution operators.

The prototype integral operator for this section is given through

$$
(L \psi)(x)=\int_{\mathbb{R}^{d}} l(x, y) \psi(y) d y, \quad x \in \mathbb{R}^{d},
$$

where we suppose that $l: \mathbb{R}^{d} \times \mathbb{R}^{d} \rightarrow \mathbb{C}$ is such that $l(x, \cdot)$ is measurable for all $x \in \mathbb{R}^{d}$ and the integral exists a.e. for an appropriately chosen $\psi$.

LEMMA 1.44. If the kernel function of the operator L, given through (1.49), can be written as

$$
l(x, y)=\ell(x-y)
$$

where $\ell \in L^{2}\left(\mathbb{R}^{d}\right)$ such that $\mathcal{F}_{2} \ell \in L^{\infty}\left(\mathbb{R}^{d}\right)$, then $L \psi=\ell * \psi$ is a bounded operator on $L^{2}\left(\mathbb{R}^{d}\right)$ with the bound

$$
\|L\|_{L^{2} \rightarrow L^{2}} \leq(2 \pi)^{d / 2}\left\|\mathcal{F}_{2} \ell\right\|_{L^{\infty}}
$$

and furthermore $L \psi \in X_{\infty}\left(\mathbb{R}^{d}\right)=L^{2}\left(\mathbb{R}^{d}\right) \cap B C_{\infty}\left(\mathbb{R}^{d}\right)$. 
Proof. We first note that $\ell \in L^{2}$ and $\mathcal{F}_{2} \ell \in L^{\infty}$ imply that $\mathcal{F}_{2} \ell \in L^{2} \cap L^{\infty}$. Now we can apply the Hölder inequality $(1.5)$ in the case $p=q=2$ to get $\mathcal{F}_{2} \ell \cdot \mathcal{F}_{2} \psi \in L^{1}$ and the generalised Hölder inequality (1.6) in the case $p=\infty, q=2, r=2$ which yields $\mathcal{F}_{2} \ell \cdot \mathcal{F}_{2} \psi \in L^{2}$; showing that $\mathcal{F}_{2} \ell \cdot \mathcal{F}_{2} \psi \in L^{1} \cap L^{2}$. If we consider $\mathcal{F}_{2} \ell \cdot \mathcal{F}_{2} \psi$ as an element of $L^{1}$, we can use (1.47) and write

$$
\ell * \psi=(2 \pi)^{d / 2} \mathcal{F}_{1}^{-1}\left(\mathcal{F}_{2} \ell \cdot \mathcal{F}_{2} \psi\right)
$$

so that the mapping properties of the $L^{1}$-Fourier transform yield $\ell * \psi \in B C_{\infty}$, see LEMMA 1.29. If on the other hand we consider $\mathcal{F}_{2} \ell \cdot \mathcal{F}_{2} \psi$ as an element of $L^{2}$, we can use (1.48) to write

$$
\ell * \psi=(2 \pi)^{d / 2} \mathcal{F}_{2}^{-1}\left(\mathcal{F}_{2} \ell \cdot \mathcal{F}_{2} \psi\right)
$$

so that the mapping properties of the Fourier-Plancherel transform yield $\ell * \psi \in L^{2}$; hence $L \psi \in L^{2} \cap B C_{\infty}$. Now we use (1.48) again to estimate

$$
\begin{aligned}
\|L \psi\|_{L^{2}} & =(2 \pi)^{d / 2}\left\|\mathcal{F}_{2}^{-1}\left(\mathcal{F}_{2} \ell \cdot \mathcal{F}_{2} \psi\right)\right\|_{L^{2}} \\
& =(2 \pi)^{d / 2}\left\|\mathcal{F}_{2} \ell \cdot \mathcal{F}_{2} \psi\right\|_{L^{2}} \\
& \leq(2 \pi)^{d / 2}\left\|\mathcal{F}_{2} \ell\right\|_{L^{\infty}}\left\|\mathcal{F}_{2} \psi\right\|_{L^{2}} \\
& =(2 \pi)^{d / 2}\left\|\mathcal{F}_{2} \ell\right\|_{L^{\infty}}\|\psi\|_{L^{2}} .
\end{aligned}
$$

REMARK 1.45. It is clear that the additional assumption $\mathcal{F} \ell \in L^{\infty}$ would have followed immediately, if we had assumed that $\ell$ was in the intersection of $L^{1}$ and $L^{2}$. However, for the application we have in mind, namely the proof of LEMMA 2.3, this assumption is not valid.

Similar estimates hold in the case of integral operators with kernels of more general type as we show now.

LEMMA 1.46. If the kernel function of the operator L, given through (1.49), can be written as

$$
l(x, y)=m_{1}(x) \ell(x-y) m_{2}(y),
$$

with $m_{1}, m_{2} \in B C\left(\mathbb{R}^{d}\right), \ell \in L^{2}\left(\mathbb{R}^{d}\right)$ and $\mathcal{F}_{2} \ell \in L^{\infty}\left(\mathbb{R}^{d}\right)$, then $L$ is a bounded operator on $L^{2}\left(\mathbb{R}^{d}\right)$ with norm

$$
\|L\|_{L^{2} \rightarrow L^{2}} \leq(2 \pi)^{d / 2}\left\|m_{1}\right\|_{B C}\left\|\mathcal{F}_{2} \ell\right\|_{L^{\infty}}\left\|m_{2}\right\|_{B C}
$$

and $L \psi \in X_{\infty}\left(\mathbb{R}^{d}\right)$.

Proof. This is a direct consequence of LEMMA 1.44 . 
Lemma 1.47. Assume for some $p \in[1, \infty)$ there exists $\ell \in L^{p}\left(\mathbb{R}^{2}\right)$ such that

$$
|l(x, y)| \leq \ell(x-y)
$$

where $l$ denotes the kernel function of the operator $L$ given through (1.49). Then $L$ is a bounded operator from $L^{q}\left(\mathbb{R}^{d}\right)$ to $L^{s}\left(\mathbb{R}^{d}\right)$ provided that $1 / p+1 / q=1+1 / s$. Furthermore one has the norm estimate

$$
\|L\|_{L^{q} \rightarrow L^{s}} \leq\|\ell\|_{L^{p}}
$$

Proof. One computes

$$
|(L \psi)(x)| \leq \int_{\mathbb{R}^{d}}|l(x, y)||\psi(y)| d y \leq \int_{\mathbb{R}^{d}} \ell(x-y)|\psi(y)| d y=(\ell *|\psi|)(x)
$$

so the mapping properties are a result from Youngs inequality (1.45).

REMARK 1.48. We will use the bound (1.52) particularly often in the case $\ell \in$ $L^{1}\left(\mathbb{R}^{2}\right)$, in which case it implies that $L$ maps $L^{q}$ to $L^{q}$ with

$$
\|L\|_{L^{q} \rightarrow L^{q}} \leq\|\ell\|_{L^{1}}
$$

for $1 \leq q \leq \infty$

Lemma 1.49. Assume for some $p \in(1,2)$ there exists $\ell \in L^{1}\left(\mathbb{R}^{2}\right) \cap L^{p}\left(\mathbb{R}^{2}\right)$ such that

$$
|l(x, y)| \leq \ell(x-y)
$$

where $l$ denotes the kernel function of the operator $L$ given through (1.49).

(i) Then for some $N \in \mathbb{N}$, the operator $L^{N+1}$ is a bounded operator from $L^{2}\left(\mathbb{R}^{2}\right)$ to $L^{\infty}\left(\mathbb{R}^{2}\right)$, i.e. there exist $N$ real numbers $r_{1}, \ldots, r_{N}$ such that $2<r_{1}<\cdots<$ $r_{N-1}<\frac{p}{p-1} \leq r_{N}$ and $L^{2} \stackrel{L}{\rightarrow} L^{r_{1}} \stackrel{L}{\rightarrow} \ldots \stackrel{L}{\rightarrow} L^{r_{N}} \stackrel{L}{\rightarrow} L^{\infty}$.

(ii) $\left|L^{N+1} \psi\right|$ is bounded a.e. by a positive continuous function vanishing at infinity.

Proof. (i) Note first that $\ell \in L^{1}\left(\mathbb{R}^{2}\right) \cap L^{p}\left(\mathbb{R}^{2}\right)$ implies by Lemma 1.9 that $\ell \in L^{s}\left(\mathbb{R}^{2}\right)$ for $1<s<p$. Let $\omega:=p /(p-1)>2$ and define the finite or infinite sequence $\left(r_{j}\right)$ iteratively by

$$
r_{0}:=2, \quad r_{j+1}:=\left(\frac{1}{p}+\frac{1}{r_{j}}-1\right)^{-1}=\frac{r_{j}}{1-\frac{r_{j}}{\omega}}, \quad j=0,1,2, \ldots,
$$

continuing the definition (1.55) for as long as $r_{j}<\omega$. Let $J \subset \mathbb{N}_{0}:=\mathbb{N} \cup\{0\}$ denote the set of indices $j$ for which $r_{j}$ is defined. We will show that the set $J$ 
is finite, so that $J=\{0,1, \ldots, N\}$ with $r_{N} \geq \omega$. Then it follows from Youngs inequality (THEOREM 1.40) that $L$ is a bounded operator from $L^{r_{j-1}}\left(\mathbb{R}^{2}\right)$ to $L^{r_{j}}\left(\mathbb{R}^{2}\right)$ for $j=1, \ldots, N$. Further, defining $s:=r_{N} /\left(r_{N}-1\right)$, we observe that $1<s<p$ and $\frac{1}{s}+\frac{1}{r_{N}}=1$, so that again, by Youngs inequality (THEOREM 1.40), $L$ is a bounded operator from $L^{r_{N}}\left(\mathbb{R}^{2}\right)$ to $L^{\infty}\left(\mathbb{R}^{2}\right)$, and so $L^{N+1}$ is a bounded operator from $L^{2}\left(\mathbb{R}^{2}\right)$ to $L^{\infty}\left(\mathbb{R}^{2}\right)$.

We complete the proof by showing that $J$ is finite. Suppose otherwise. Then $r_{j}<\omega$ for all $j \in J=\mathbb{N}_{0}$. It follows from (1.55), by induction, that the sequence $\left(r_{j}\right)$ is monotonically increasing. Thus the sequence $\left(r_{j}\right)$ is convergent to some limit $r$, with $2 \leq r \leq \omega$. Rearranging (1.55) and taking limits, we see that $(1-r / \omega) r=r$, so that $r=0$, a contradiction.

(ii) It follows by induction that $\left|L^{N+1} \psi\right| \leq \ell *\left|L^{N} \psi\right|$ for $N \in \mathbb{N}$. From the construction of the sequence $\left(r_{j}\right)$, see above, we have $\ell \in L^{s}, L^{N} \psi \in L^{r_{N}}$ with $\frac{1}{s}+\frac{1}{r_{N}}=1$. Thus it follows from THEOREM 1.40 (iii), that

$$
\left|L^{N+1} \psi\right| \leq \ell *\left|L^{N} \psi\right| \in B C_{\infty}\left(\mathbb{R}^{2}\right)
$$

i.e. $\left|L^{N+1} \psi\right|$ is bounded a.e. by a continuous function vanishing at infinity. 


\section{Chapter 2}

\section{Properties of single- and double-layer potentials}

In this chapter we prove mapping properties of the double- and single-layer potential operators, verify the validity of the standard jump-relations and prove continuity properties of the boundary operators with respect to variations of the scattering surface and the wavenumber.

To prove mapping properties we split the operators into a local and a global part with the help of an appropriate cut-off function. To this end let $\chi:[0, \infty) \rightarrow \mathbb{R}$ be a sufficiently smooth function that satisfies the global lower and upper bound

$$
0 \leq \chi(t) \leq 1, \quad t \geq 0
$$

and the condition

$$
\chi(t)=\left\{\begin{array}{ll}
1, & t<1 / 2 \\
0, & t \geq 1
\end{array} .\right.
$$

Following the explanation at the end of Section 1.1.3 we show the mapping properties for the associated operator

$$
\tilde{B}:=I_{f} B I_{f}^{-1} \in B L\left(L^{2}\left(\mathbb{R}^{2}\right)\right),
$$

where $B$ denotes one of the operators $S$ or $K$, respectively. Thus

$$
(\tilde{B} \psi)(\boldsymbol{x}):=\int_{\mathbb{R}^{2}} \tilde{b}(\boldsymbol{x}, \boldsymbol{y}) \psi(\boldsymbol{y}) d \boldsymbol{y}, \quad \boldsymbol{x} \in \mathbb{R}^{2},
$$

where the kernel function is given, in the case of the single layer operator, by

$$
\tilde{b}(\boldsymbol{x}, \boldsymbol{y})=2 G(x, y) J(\boldsymbol{y})
$$

and, in the case of the double layer potential, through

$$
\tilde{b}(\boldsymbol{x}, \boldsymbol{y})=2 \frac{\partial G(x, y)}{\partial \nu(y)} J(\boldsymbol{y}),
$$


with $x=(\boldsymbol{x}, f(\boldsymbol{x})), y=(\boldsymbol{y}, f(\boldsymbol{y})) \in \Gamma$ and $J(\boldsymbol{y})=\sqrt{1+|\nabla f(\boldsymbol{y})|^{2}}$.

We define the global part of the operator

$$
\left(\tilde{B}_{\text {global }} \psi\right)(\boldsymbol{x}):=\int_{\mathbb{R}^{2}}[1-\chi(|\boldsymbol{x}-\boldsymbol{y}|)] \tilde{b}(\boldsymbol{x}, \boldsymbol{y}) \psi(\boldsymbol{y}) d \boldsymbol{y}, \quad \boldsymbol{x} \in \mathbb{R}^{2},
$$

and the local part

$$
\left(\tilde{B}_{\text {local }} \psi\right)(\boldsymbol{x}):=\int_{\mathbb{R}^{2}} \chi(|\boldsymbol{x}-\boldsymbol{y}|) \tilde{b}(\boldsymbol{x}, \boldsymbol{y}) \psi(\boldsymbol{y}) d \boldsymbol{y}, \quad \boldsymbol{x} \in \mathbb{R}^{2} .
$$

This yields the decomposition $\tilde{B}=\tilde{B}_{\text {global }}+\tilde{B}_{\text {local }}$ which allows us to study the mapping properties of $\tilde{B}_{\text {global }}$ and $\tilde{B}_{\text {local }}$ as operators on $L^{2}\left(\mathbb{R}^{2}\right), X\left(\mathbb{R}^{2}\right)$ and $X_{\infty}\left(\mathbb{R}^{2}\right)$ separately.

\subsection{Boundedness of $S$ and $K$}

We start this section with a series of Lemmata that are needed to prove that the single- and double-layer potential operators are bounded on any of the function spaces $L^{2}, X$ and $X_{\infty}$. To prove this result we prove the corresponding results for the local and global part of the operators $S$ and $K$ separately by using the expansions (1.21) and (1.27) and the results on convolution operators from Section 1.6.1.

LEMMA 2.1 (Mapping properties for the local part). $\tilde{B}_{\text {local }}$ is a bounded operator from

(i) $L^{\infty}\left(\mathbb{R}^{2}\right)$ to $B C\left(\mathbb{R}^{2}\right)$,

(ii) $B C_{\infty}\left(\mathbb{R}^{2}\right)$ to $B C_{\infty}\left(\mathbb{R}^{2}\right)$,

(iii) $L^{q}\left(\mathbb{R}^{2}\right)$ to $L^{q}\left(\mathbb{R}^{2}\right)$ for $1<q<\infty$.

(iv) Further, for some $n \in \mathbb{N}$, $\tilde{B}_{\text {local }}^{n}$ is a bounded operator from $L^{2}\left(\mathbb{R}^{2}\right)$ to $X\left(\mathbb{R}^{2}\right)$ and from $X\left(\mathbb{R}^{2}\right)$ to $X_{\infty}\left(\mathbb{R}^{2}\right)$.

Proof. We start this proof with a series of observations on the kernel function $\tilde{b}_{\text {local }}$ of the operator $\tilde{B}_{\text {local }}$ :

- The kernel has compact support, more precisely it holds that

$$
\operatorname{supp} \tilde{b}_{\text {local }}(\boldsymbol{x}, \cdot) \subset B_{1}(\boldsymbol{x}), \quad \boldsymbol{x} \in \mathbb{R}^{2} .
$$

- It is continuous for $\boldsymbol{x} \neq \boldsymbol{y}$ and weakly singular. Precisely, it follows from LEMMA 1.17 that

$$
|\langle\nu(y), x-y\rangle| \leq|\boldsymbol{x}-\boldsymbol{y}|^{1+\alpha}\|f\|_{B C^{1, \alpha}\left(\mathbb{R}^{2}\right)}, \quad x, y \in \Gamma,
$$


thus it holds in the double-layer case $B=K$ that, for some constant $C>0$,

$$
\left|\tilde{b}_{\text {local }}(\boldsymbol{x}, \boldsymbol{y})\right|<\ell(\boldsymbol{x}-\boldsymbol{y}), \quad \boldsymbol{x}, \boldsymbol{y} \in \mathbb{R}^{2}, \boldsymbol{x} \neq \boldsymbol{y},
$$

where

$$
\ell(\boldsymbol{y}):= \begin{cases}C|\boldsymbol{y}|^{\alpha-2}, & |\boldsymbol{y}| \leq 1 \\ 0, & |\boldsymbol{y}|>1 .\end{cases}
$$

The same bound holds, but is not sharp, in the single-layer case $B=S$. We showed in EXAMPLE 1.10 that $\ell \in L^{1}\left(\mathbb{R}^{2}\right) \cap L^{p}\left(\mathbb{R}^{2}\right)$ for $1 \leq p<\frac{2}{2-\alpha}<2$.

Thus we note that the kernel function $\tilde{b}_{\text {local }}$ satisfies all the requirements to apply Lemma 1.15, Lemma 1.47 and Lemma 1.49. Now we can prove the Lemma:

(i) and (ii) follow directly from LEMMA 1.15.

(iii) As $\ell \in L^{1}$, it follows from LemMA 1.47, in the case $p=1$, that $\tilde{B}_{\text {local }}$ is a bounded operator from $L^{q}\left(\mathbb{R}^{2}\right)$ to $L^{q}\left(\mathbb{R}^{2}\right)$ for $1<q<\infty$.

(iv) It follows from (iii) that $\tilde{B}_{\text {local }}^{n}$ maps $L^{2}$ to $L^{2}$ for $n \in \mathbb{N}$. On the other hand by LEMma $1.49, \tilde{B}_{\text {local }}^{m}$ is a bounded operator from $L^{2}$ to $L^{\infty}$, for some $m \in \mathbb{N}$. From LEMMA 1.15 it follows that $\tilde{B}_{\text {local }}$ is a bounded operator from $L^{\infty}$ to $B C$, so that altogether $\tilde{B}_{\text {local }}^{m+1}$ is a bounded operator from $L^{2}$ to $X$.

It follows from LEMmA 1.49 that $\tilde{B}_{\text {local }}^{m}$ is a bounded operator from $L^{2}$ to $L^{\infty}$, for some $m \in \mathbb{N}$ and furthermore that $\tilde{B}_{\text {local }}^{m} \psi$ is bounded a.e. by a positive function $\Psi \in B C_{\infty}$. By Lemma $1.15 \tilde{B}_{\text {local }}^{m}$ maps $B C$ to $B C$ for any $m \in \mathbb{N}$, hence $\tilde{B}_{\text {local }}^{m}$ maps $X$ to $B C_{\infty}$.

Combining the above mapping properties we derive the following corollary.

COROLlaRY 2.2. $\tilde{B}_{\text {local }}$ is a bounded operator from

(i) $B C\left(\mathbb{R}^{2}\right)$ to $B C\left(\mathbb{R}^{2}\right)$,

(ii) $X\left(\mathbb{R}^{2}\right)$ to $X\left(\mathbb{R}^{2}\right)$,

(iii) $X_{\infty}\left(\mathbb{R}^{2}\right)$ to $X_{\infty}\left(\mathbb{R}^{2}\right)$.

Proof. (i) is a direct consequence of LEMMA 2.1 (i). (ii) follows from combining (i) and LEMma 2.1 (iii) for $q=2$. (iii) follows from combining LEMMA 2.1 (ii) and LEMMA 2.1 (iii) for $q=2$.

We now consider the global part and prove a lemma on the mapping properties of $\tilde{B}_{\text {global }}$.

LEMMA 2.3 (Mapping properties for the global part). $\tilde{B}_{\text {global }}$ is a bounded operator from 
(i) $L^{2}\left(\mathbb{R}^{2}\right)$ to $L^{2}\left(\mathbb{R}^{2}\right)$,

(ii) $L^{2}\left(\mathbb{R}^{2}\right)$ to $X\left(\mathbb{R}^{2}\right)$,

(iii) $L^{2}\left(\mathbb{R}^{2}\right)$ to $X_{\infty}\left(\mathbb{R}^{2}\right)$.

Proof. We start the proof of this Lemma by proving the following claim.

Claim: The kernel $\tilde{b}_{\text {global }}$ of $\tilde{B}_{\text {global }}$ can be written, in both the cases $B=S$ and $B=K$, in the form

$$
\tilde{b}_{\text {global }}(\boldsymbol{x}, \boldsymbol{y})=l^{*}(\boldsymbol{x}, \boldsymbol{y})+l(\boldsymbol{x}, \boldsymbol{y}), \quad \boldsymbol{x}, \boldsymbol{y} \in \mathbb{R}^{2},
$$

where $l^{*}$ is a sum of terms each of the form (1.50), with $m_{1}, m_{2} \in B C\left(\mathbb{R}^{2}\right)$ and $\ell$ given by (1.33) with $\beta=1$, and with $n=-1,0,1$. Further, $l^{*}$ can be chosen so that $l$ satisfies the bound

$$
|l(\boldsymbol{x}, \boldsymbol{y})|<C \tilde{\ell}(\boldsymbol{x}-\boldsymbol{y}), \quad \boldsymbol{x}, \boldsymbol{y} \in \mathbb{R}^{2},
$$

for some constant $C>0$, where $\tilde{\ell}(\boldsymbol{y}):=(1+|\boldsymbol{y}|)^{-3}$, so that $\tilde{\ell} \in L^{1}\left(\mathbb{R}^{2}\right)$.

Proof of Claim. The proof follows directly from the decompositions (1.21) and (1.27). In detail, in the case $B=S$ we see from (1.21) that an appropriate choice is to take

$$
l^{*}(\boldsymbol{x}, \boldsymbol{y})=-\frac{i \kappa}{2 \pi} f(\boldsymbol{x}) \frac{e^{i \kappa|\boldsymbol{x}-\boldsymbol{y}|}}{1+|\boldsymbol{x}-\boldsymbol{y}|^{2}} f(\boldsymbol{y}) J(\boldsymbol{y}),
$$

where $J$ is the surface area element given through (1.10), while, in the case $B=K$ we see from (1.27) that we can take

$$
\begin{aligned}
l^{*}(\boldsymbol{x}, \boldsymbol{y}) & =-\frac{\kappa^{2}}{2 \pi} f(\boldsymbol{x}) \frac{e^{i \kappa|\boldsymbol{x}-\boldsymbol{y}|}}{1+|\boldsymbol{x}-\boldsymbol{y}|^{2}} \boldsymbol{\nu}(y) \cdot \frac{\boldsymbol{x}-\boldsymbol{y}}{|\boldsymbol{x}-\boldsymbol{y}|} f(\boldsymbol{y}) J(\boldsymbol{y}) \\
& -\frac{i \kappa}{2 \pi} f(\boldsymbol{x}) \frac{e^{i \kappa|\boldsymbol{x}-\boldsymbol{y}|}}{1+|\boldsymbol{x}-\boldsymbol{y}|^{2}} \nu_{3}(y) J(\boldsymbol{y}) .
\end{aligned}
$$

To see more clearly that this is an expression of the required form we recall that

$$
\nu(y)=\left(\boldsymbol{\nu}(y), \nu_{3}(y)\right)=\frac{(-\nabla f(\boldsymbol{y}), 1)}{J(\boldsymbol{y})}
$$

so that we can write

$$
\boldsymbol{\nu}(y) \cdot \frac{\boldsymbol{x}-\boldsymbol{y}}{|\boldsymbol{x}-\boldsymbol{y}|}=\frac{|\nabla f(\boldsymbol{y})|}{J(\boldsymbol{y})} \cos \theta
$$


where $\theta=\angle(\nabla f(\boldsymbol{y}), \boldsymbol{x}-\boldsymbol{y})$. Furthermore using the identity $\cos =\left(e^{i \theta}+e^{-i \theta}\right) / 2$ we have

$$
\begin{aligned}
l^{*}(\boldsymbol{x}, \boldsymbol{y}) & =-\frac{\kappa^{2}}{4 \pi} f(\boldsymbol{x}) \frac{e^{i \kappa|\boldsymbol{x}-\boldsymbol{y}|}}{1+|\boldsymbol{x}-\boldsymbol{y}|^{2}} e^{i \theta}|\nabla f(\boldsymbol{y})| f(\boldsymbol{y}) \\
& -\frac{\kappa^{2}}{4 \pi} f(\boldsymbol{x}) \frac{e^{i \kappa|\boldsymbol{x}-\boldsymbol{y}|}}{1+|\boldsymbol{x}-\boldsymbol{y}|^{2}} e^{-i \theta}|\nabla f(\boldsymbol{y})| f(\boldsymbol{y}) \\
& -\frac{i \kappa}{2 \pi} f(\boldsymbol{x}) \frac{e^{i \kappa|\boldsymbol{x}-\boldsymbol{y}|}}{1+|\boldsymbol{x}-\boldsymbol{y}|^{2}} .
\end{aligned}
$$

This proves the claim.

Now we prove the Lemma. (i) It follows from Lemma 1.46 and LEMmA 1.33 applied to the integral operator with kernel $l^{*}$, and (1.54) applied to the integral operator with kernel $l$, that $\tilde{B}_{\text {global }}$ is a bounded operator on $L^{2}\left(\mathbb{R}^{2}\right)$.

(ii) and (iii) We note from the above representation (2.7) that also

$$
\left|\tilde{b}_{\text {global }}(x, y)\right| \leq \ell^{*}(\boldsymbol{x}-\boldsymbol{y}), \quad x, y \in \Gamma,
$$

for some $\ell^{*} \in L^{2}\left(\mathbb{R}^{2}\right)$; this is true since $\tilde{\ell} \in L^{2}\left(\mathbb{R}^{2}\right)$ and since each term of $(2.9)$ and (2.10) can be bounded in this way. It follows from Youngs inequality (THEOREM 1.40) that $\tilde{B}_{\text {global }}$ maps $L^{2}\left(\mathbb{R}^{2}\right)$ to $L^{\infty}\left(\mathbb{R}^{2}\right)$; in fact, since also $\tilde{b}_{\text {global }}$ is continuous, it holds that $\tilde{B}_{\text {global }}$ maps $L^{2}\left(\mathbb{R}^{2}\right)$ to $B C\left(\mathbb{R}^{2}\right)$. Furthermore it follows from THEOREM 1.40 (iii) that $\tilde{B}_{\text {global }}$ maps $L^{2}\left(\mathbb{R}^{2}\right)$ to $B C_{\infty}\left(\mathbb{R}^{2}\right)$.

Combining the above mapping properties we have the following Corollary.

Corollary 2.4. $\tilde{B}_{\text {global }}$ is a bounded operator on $X\left(\mathbb{R}^{2}\right)$ and on $X_{\infty}\left(\mathbb{R}^{2}\right)$.

REMARK 2.5. For $C_{2}>C_{1}>0$ let

$$
B\left(C_{1}, C_{2}\right):=\left\{f \in B C^{1, \alpha}\left(\mathbb{R}^{2}\right): C_{1} \leq f(\boldsymbol{y}), \boldsymbol{y} \in \mathbb{R}^{2}, \quad\|f\|_{B C^{1, \alpha}\left(\mathbb{R}^{2}\right)} \leq C_{2}\right\} .
$$

We note that, given $C_{2}>C_{1}>0$ and $\kappa_{r}>0$, we can choose $C>0$ such that the estimates (2.5) and (2.8) hold for all $f \in B$ and all $\kappa_{i} \geq 0$. (For (2.8) this follows from (1.21) and (1.27).) This observation will be helpful in establishing continuous dependence of $S$ and $K$ on $f$ and on $\kappa_{i}$. Furthermore we see that $f \in B\left(C_{1}, C_{2}\right)$ implies that

$$
C_{1} \leq f(\boldsymbol{y}) \leq C_{2}, \quad \boldsymbol{y} \in \mathbb{R}^{2} .
$$

This follows from the fact that in the case of a constant function $f_{h}$ with $h>0$ we have that $\left\|f_{h}\right\|_{B C^{1, \alpha}\left(\mathbb{R}^{2}\right)}=h$.

We can now combine all the previous Lemmata to give a prove of one of the main results of this chapter. 
THEOREM 2.6. The single- and double-layer potential operators $S$ and $K$, defined by (12) and (13), are bounded operators on $L^{2}(\Gamma)$, on $X(\Gamma)$ and on $X_{\infty}(\Gamma)$.

Proof. Lemma 2.1 and Corollary 2.2 yield that $\tilde{B}_{\text {local }}$ is a bounded operator on $L^{2}\left(\mathbb{R}^{2}\right), X\left(\mathbb{R}^{2}\right)$ and $X_{\infty}\left(\mathbb{R}^{2}\right)$; LEMma 2.3 and Corollary 2.4 yield that $\tilde{B}_{\text {global }}$ is a bounded operator on $L^{2}\left(\mathbb{R}^{2}\right), X\left(\mathbb{R}^{2}\right)$ and $X_{\infty}\left(\mathbb{R}^{2}\right)$, hence finishing the proof.

Yet another consequence is:

Corollary 2.7. For all sufficiently large $n \in \mathbb{N}$ it holds that $B^{n}$, where $B$ denotes either $B=S$ or $B=K$, is a bounded map from $L^{2}(\Gamma)$ to $X(\Gamma)$ and from $X(\Gamma)$ to $X_{\infty}(\Gamma)$.

As part of the proof of THEOREM 3.1 on the equivalence of the integral equation and the boundary value problem we need to show that the modified single- and double-layer potentials $u_{1}$ and $u_{2}$, over the unbounded surface $\Gamma$, behave in a similar way to the corresponding standard layer potentials supported on a smooth bounded surface. This is done in the following theorem in which

$$
M:=\left\{x: 0<x_{3}<f(\boldsymbol{x})\right\}
$$

denotes the region between $\Gamma$ and $\Gamma^{0}$.

THEOREM 2.8. Let $u_{1}$ and $u_{2}$ denote the single- and double-layer potentials with density $\varphi \in X$, defined by (16) and (17), respectively. It holds that:

(i) For $n=1,2, u_{n} \in C^{2}(D \cup M)$ and $\Delta u_{n}+k^{2} u_{n}=0$ in $D \cup M$.

(ii) $u_{1}$ and $u_{2}$ can be continuously extended from $D$ to $\bar{D}$ and from $M$ to $\bar{M}$, with limiting values

$$
u_{1, \pm}(x)=\int_{\Gamma} G(x, y) \varphi(y) d s(y), \quad x \in \Gamma
$$

and

$$
u_{2, \pm}(x)=\int_{\Gamma} \frac{\partial G(x, y)}{\partial \nu(y)} \varphi(y) d s(y) \pm \frac{1}{2} \varphi(x), \quad x \in \Gamma,
$$

where $u_{n, \pm}(x):=\lim _{\epsilon \rightarrow 0+} u_{n}(x \pm \epsilon \nu(x))$, for $n=1,2$ and $x \in \Gamma$, and $\nu(x)$ denotes the unit normal at $x \in \Gamma$ directed into $D$.

(iii) Given constants $C_{2}>C_{1}>0$ and a compact subset $S \subset \tilde{\mathbb{C}}$, there exists a constant $C>0$ such that

$$
\left|u_{n}(x)\right| \leq C\|\varphi\|_{X}, \quad x \in D \cup M, n=1,2,
$$

for all $\varphi \in X, \kappa \in S$, and $f \in B=B\left(C_{1}, C_{2}\right)$. 
(iv) Given constants $C_{2}>C_{1}>0$ and $\epsilon>0$ and a compact subset $S \subset \tilde{\mathbb{C}}$, there exits a constant $C>0$ such that

$$
\left|u_{n}(x)\right| \leq C\|\varphi\|_{L^{2}(\Gamma)}, \quad n=1,2,
$$

for all $x \in D \cup M$ with $\left|x_{3}-f\left(x_{1}, x_{2}\right)\right|>\epsilon$, all $\varphi \in X$, all $\kappa \in S$, and all $f \in B=$ $B\left(C_{1}, C_{2}\right)$.

Proof. We first of all show that $u \in C(D \cup M)$ and establish (ii) and (iii). We use the cut-off function $\chi$ given by (2.1) and (2.2).

Let $u$ denote one of $u_{1}$ and $u_{2}$, and let $b$ denote the kernel of $u$ so that $b(x, y):=$ $G(x, y)$ and $b(x, y):=\partial G(x, y) / \partial \nu(y)$ in the respective cases. We have, for $x \in$ $D \cup M$, that

$$
u(x)=\int_{\Gamma}[1-\chi(|x-y|)] b(x, y) \varphi(y) d s(y)+\int_{\Gamma} \chi(|x-y|) b(x, y) \varphi(y) d s(y) .
$$

The first term has a continuous kernel that is bounded at infinity by the estimate (1.21) or (1.25), and, since $\varphi \in L^{2}(\Gamma)$, is continuous in $\left\{x: x_{3}>0\right\}$. The second term is clearly continuous in $D \cup M$; to see that it can be continuously extended up to $\Gamma$ from above and below and to compute its limiting values we observe that, keeping $x$ within some ball centred on some $x_{0} \in \Gamma$, it holds that the integrand is supported in a finite patch of the surface. We can extend this surface patch to a bounded obstacle with boundary of class $C^{1, \alpha}$ and, since $\varphi \in C(\Gamma)$, use the jump relations for bounded obstacles THEOREM 1.20 and thus finishing the proof of (ii).

(iii) To show that the first term satisfies the bound (2.15) we recall that $G(x, y)$ and $\nabla_{y} G(x, y)$ satisfy the bound

$$
|G(x, y)|,\left|\nabla_{y} G(x, y)\right| \leq C \frac{\left(1+x_{3}\right)\left(1+y_{3}\right)}{|x-y|^{2}},
$$

with a possibly different constant $C$, for all $x, y \in D^{0}=\left\{x \in \mathbb{R}^{3}: x_{3}>0\right\}$ with $|x-y| \geq 1$ and all $\kappa \in S$, cf. Lemma 1.22 and LEMma 1.25.

Thus, for some constant $C^{\prime}>0$, whether $b$ is the kernel of the single- or doublelayer potential, it holds for all $\kappa \in S$ that

$$
|[1-\chi(|x-y|)] b(x, y)| \leq C^{\prime} \frac{\left(1+x_{3}\right)\left(1+y_{3}\right)}{1+|x-y|^{2}}, \quad x, y \in \mathbb{R}^{3}, x_{3}, y_{3} \geq 0 .
$$

Bounding the global part: Applying the Cauchy-Schwarz inequality together with the bound (2.17) we calculate for the first term that

$$
\begin{aligned}
\left|\int_{\Gamma}[1-\chi(|x-y|)] b(x, y) \varphi(y) d s(y)\right| & \leq C^{\prime}\left(\int_{\Gamma} \frac{\left(1+x_{3}\right)^{2}\left(1+y_{3}\right)^{2}}{\left(1+|x-y|^{2}\right)^{2}} d s(y)\right)^{1 / 2}\|\varphi\|_{L^{2} \Gamma} \\
& \leq C^{\prime}\left(1+f_{+}\right) I(x)\|\varphi\|_{L^{2}(\Gamma)},
\end{aligned}
$$


for $x \in\left\{x \in \mathbb{R}^{3}: x_{3}>0\right\}$, where

$$
[I(x)]^{2}=\int_{\Gamma} \frac{\left(1+x_{3}\right)^{2}}{\left(1+|x-y|^{2}\right)^{2}} d s(y) .
$$

We further estimate

$$
[I(x)]^{2} \leq\left(1+\|\nabla f\|_{B C(\Gamma)}^{2}\right)^{1 / 2} \int_{\mathbb{R}^{2}} \frac{\left(1+x_{3}\right)^{2}}{\left(1+|\boldsymbol{x}-\boldsymbol{y}|^{2}+\left(x_{3}-f(\boldsymbol{y})\right)^{2}\right)^{2}} d \boldsymbol{y} .
$$

To finally prove the bound for the global part we show that the function

$$
F\left(x_{3}\right):=\int_{\mathbb{R}^{2}} \frac{\left(1+x_{3}\right)^{2}}{\left(1+|\boldsymbol{x}-\boldsymbol{y}|^{2}+\left(x_{3}-f(\boldsymbol{y})\right)^{2}\right)^{2}} d \boldsymbol{y},
$$

is bounded on $[0, \infty)$ for all $f \in B$. We note that $F$ is continuous on $[0, \infty)$ and recall from REMARK 2.5 that $C_{1} \leq f(\boldsymbol{y}) \leq C_{2}$ for all $f \in B\left(C_{1}, C_{2}\right)$ and $\boldsymbol{y} \in \mathbb{R}^{2}$. Thus we have the lower bound

$$
\left[\delta\left(x_{3}\right)\right]^{2} \leq\left(x_{3}-f(\boldsymbol{y})\right)^{2}, \quad x_{3} \in[0, \infty),
$$

for all $f \in B\left(C_{1}, C_{2}\right)$ and $\boldsymbol{y} \in \mathbb{R}^{2}$, where

$$
\delta(t):= \begin{cases}C_{1}-t, & 0 \leq t \leq C_{1} \\ 0, & C_{1} \leq t \leq C_{2} \\ t-C_{2}, & t \geq C_{2}\end{cases}
$$

Thus

$$
F\left(x_{3}\right) \leq \int_{\mathbb{R}^{2}} \frac{\left(1+x_{3}\right)^{2}}{\left(1+|\boldsymbol{x}-\boldsymbol{y}|^{2}+\left[\delta\left(x_{3}\right)\right]^{2}\right.} d \boldsymbol{y} \leq 2 \pi \int_{0}^{\infty} \frac{\left(1+x_{3}\right)^{2} r}{\left(1+r^{2}+\left[\delta\left(x_{3}\right)\right]^{2}\right)^{2}} d r .
$$

From this we see that $F(0)<\infty$ and furthermore for any $x_{3}>C_{2}$ we have the estimate

$$
\begin{aligned}
F\left(x_{3}\right) & \leq 2 \pi\left(1+x_{3}\right)^{2} \int_{0}^{\infty} \frac{r}{\left(1+r^{2}+\left(x_{3}-C_{2}\right)^{2}\right)^{2}} d r \\
& =2 \pi \frac{\left(1+x_{3}\right)^{2}}{\left(x_{3}-C_{2}\right)^{2}} \int_{0}^{\infty} \frac{s}{\left(\left(x_{3}-C_{2}\right)^{-2}+1+s^{2}\right)^{2}} d s<\infty .
\end{aligned}
$$

Together with the continuity of $F$ it follows that $F$ is bounded on $[0, \infty)$ and hence the first term satisfies the bound (2.15).

Bounding the local part: To treat the second term we argue analogously to the corresponding 2D case [16]. We remark that $\chi(|x-y|)$ is zero for $|x-y| \geq 1$. We consider only the double-layer case $u=u_{1}$ (the argument is similar but simpler 
in the single-layer case). Directly from the definitions (see (1.25)) we see that there exists a constant $C>0$ such that

$$
|\chi(|x-y|) b(x, y)| \leq C \frac{|\nu(y) \cdot(x-y)|}{|x-y|^{3}}, \quad x \in D \cup M, y \in \Gamma,
$$

for all $\kappa \in S$ and all $f \in B$. For $x=\left(\boldsymbol{x}, x_{3}\right) \in D \cup M$ define $x^{*}:=(\boldsymbol{x}, f(\boldsymbol{x})) \in \Gamma$ and $x_{0}:=(\boldsymbol{x}, f(\boldsymbol{y}))$. As a short hand notation we introduce $\delta:=\left|x^{*}-x\right|=\left|f(\boldsymbol{x})-x_{3}\right|$ and note that $x_{0}-x \perp x-y$. Thus we conclude from the Pythagorean theorem that

$$
\left|x_{0}-x\right|^{2}=|x-y|^{2}-\left|x_{0}-y\right|^{2} \leq|x-y|^{2}, \quad x \in D \cup M, y \in \Gamma .
$$

Using the triangle inequality, Pythagora's theorem, the above inequality and the mean-value theorem we conclude that

$$
\begin{aligned}
\left(|\boldsymbol{x}-\boldsymbol{y}|^{2}+\delta^{2}\right)^{1 / 2} & =\left(\left|x_{0}-y\right|^{2}+\left|x^{*}-x\right|^{2}\right)^{1 / 2} \\
& \leq\left(\left|x_{0}-y\right|^{2}+\left(\left|x^{*}-x_{0}\right|+\left|x_{0}-x\right|\right)^{2}\right)^{1 / 2} \\
& =\left(\left|x_{0}-y\right|^{2}+\left|x^{*}-x_{0}\right|^{2}+2\left|x^{*}-x_{0}\right|\left|x_{0}-x\right|+\left|x_{0}-x\right|^{2}\right)^{1 / 2} \\
& =\left(\left|x_{0}-y\right|^{2}+\left|x^{*}-x_{0}\right|^{2}+2\left|x^{*}-x_{0}\right|\left|x_{0}-x\right|\right. \\
& \left.\quad \quad+|x-y|^{2}-\left|x_{0}-y\right|^{2}\right)^{1 / 2} \\
& \leq\left(\left|x^{*}-x_{0}\right|^{2}+2\left|x^{*}-x_{0}\right||x-y|+|x-y|^{2}\right)^{1 / 2} \\
& =|x-y|+\left|x^{*}-x_{0}\right|=|x-y|+|f(\boldsymbol{x})-f(\boldsymbol{y})| \\
& \leq|x-y|+\|\nabla f\|_{B C(\Gamma)}|\boldsymbol{x}-\boldsymbol{y}| \leq\left(1+\|\nabla f\|_{B C(\Gamma)}\right)|x-y| .
\end{aligned}
$$

Using this inequality, and (1.16) to bound $\left|\nu(y) \cdot\left(x^{*}-y\right)\right|$, we see that, for some $C^{\prime}>0$,

$$
|\chi(|x-y|) b(x, y)| \leq C^{\prime} \frac{|\boldsymbol{x}-\boldsymbol{y}|^{1+\alpha}+\delta}{\left(|\boldsymbol{x}-\boldsymbol{y}|^{2}+\delta^{2}\right)^{3 / 2}}, \quad x \in D \cup M, y \in \Gamma,
$$

for all $\kappa \in S$ and $f \in B$. Thus, defining $C^{\prime \prime}=C^{\prime}\left(1+\|\nabla f\|_{B C(\Gamma)}^{2}\right)^{1 / 2}$, the second term is bounded by

$$
C^{\prime \prime}\|\varphi\|_{B C(\Gamma)} \int_{|\boldsymbol{y}|<1} \frac{|\boldsymbol{y}|^{1+\alpha}+\delta}{\left(|\boldsymbol{y}|^{2}+\delta^{2}\right)^{3 / 2}} d \boldsymbol{y} \leq 2 \pi C^{\prime \prime}\|\varphi\|_{B C(\Gamma)} \int_{0}^{1} \frac{r^{1+\alpha}+\delta}{\left(r^{2}+\delta^{2}\right)^{3 / 2}} r d r,
$$

for all $\kappa \in S$ and $f \in B$. The integral remains finite also in the case $\delta \rightarrow 0$ so that the second term satisfies the bound (2.15).

To establish (iv) we modify the argument used to show (iii). We have remarked above that both $G(x, y)$ and $\nabla_{y} G(x, y)$ satisfy the bound (1.22). Thus (cf. (2.17)), for every $\epsilon>0$ there exists $C_{\epsilon}>0$ such that

$$
|b(x, y)| \leq C_{\epsilon} \frac{\left(1+x_{3}\right)\left(1+y_{3}\right)}{1+|x-y|^{2}}
$$


for all $x, y \in \mathbb{R}^{3}$ with $x_{3}, y_{3} \geq 0$ and $|x-y| \geq \epsilon$ and all $\kappa \in S$. Applying CauchySchwarz, as in the proof of (2.15), we see that it holds, for some constant $C_{\epsilon}^{\prime}>0$, that

$$
\left|u_{n}(x)\right| \leq C_{\epsilon}^{\prime}\left(1+f_{+}\right) I(x)\|\varphi\|_{L^{2}(\Gamma)}, \quad n=1,2,
$$

for all $x \in D \cup M$ with $\left|x_{3}-f\left(x_{1}, x_{2}\right)\right| \geq \epsilon$ and all $\kappa \in S$ and $f \in B$. In view of the bound on $I(x)$ already shown above, we see that we have established (2.16).

We complete the proof by establishing (i). This is clear when $\varphi$ is compactly supported. The general case follows from the density in $L^{2}(\Gamma)$ of the set of those elements of $X$ that are compactly supported, from the bound (2.16), and from the fact that limits of uniformly convergent sequences of solutions of the Helmholtz equation satisfy the Helmholtz equation (e.g. [17, Remark 2.8]).

\subsection{Continuity properties of the potential operators}

We finish this chapter with results on the continuous dependence of the potential operators on variations of the boundary and the wavenumber.

We start by proving that the single- and double-layer potential operators depend continuously on variations in the boundary $\Gamma_{f}:=\left\{(\boldsymbol{y}, f(\boldsymbol{y})): \boldsymbol{y} \in \mathbb{R}^{2}\right\}$ of the domain $D_{f}$ as defined in (3). In the statement of the following theorem, $B=B\left(C_{1}, C_{2}\right)$ is the set defined in REMARK 2.5, for some constants $C_{2}>C_{1}>0$.

To make the dependence of the operators on the scattering surface more explicit, we write $B_{f}$ for either $S$ or $K$ defined on a surface $\Gamma_{f}$ given by some $f \in B$. As before we prove the results for the associated operators $\tilde{B}_{f}=I_{f} B_{f} I_{f}^{-1} \in L^{2}\left(\mathbb{R}^{2}\right)$.

Denoting the kernel of $\tilde{B}_{f}$ by $\tilde{b}_{f}$, we see that, where $x=(\boldsymbol{x}, f(\boldsymbol{x})), y=(\boldsymbol{y}, f(\boldsymbol{y}))$, and $b(x, y):=G(x, y)$ or $b(x, y):=\partial G(x, y) / \partial \nu(y)$, in the respective cases $B_{f}=S$ and $B_{f}=K$, it holds that

$$
\tilde{b}_{f}(\boldsymbol{x}, \boldsymbol{y})=b(x, y) J_{f}(\boldsymbol{y}), \quad J_{f}(\boldsymbol{y}):=\sqrt{1+|\nabla f(\boldsymbol{y})|^{2}} .
$$

THEOREM 2.9. The single- and double-layer potential operators depend continuously on the boundary $\Gamma_{f}$ of the unbounded domain $D_{f}$ in the sense that

$$
\sup _{\| f, g \in B}\left\|\tilde{B}_{f}-\tilde{B}_{g}\right\|_{L^{2}\left(\mathbb{R}^{2}\right) \rightarrow L^{2}\left(\mathbb{R}^{2}\right)} \rightarrow 0, \quad \epsilon \rightarrow 0 .
$$

Proof. Similarly to how we proceeded when proving THEOREM 2.6, we decompose the operator $\tilde{B}_{f}-\tilde{B}_{g}$ into a global and a local part, i.e. $\tilde{B}_{f}-\tilde{B}_{g}=\tilde{B}_{\text {global }}+\tilde{B}_{\text {local }}$ with $\tilde{B}_{\text {global }}, \tilde{B}_{\text {local }}$ defined similarly to $(2.3)$ and (2.4). We now carry out the proof for the case of the single-layer operator. The necessary changes for the double-layer operator are straightforward. 
The global operator. The kernel of the global operator $\tilde{B}_{\text {global }}$ is given by

$$
\tilde{b}_{\text {global }}(\boldsymbol{x}, \boldsymbol{y}):=(1-\chi(|\boldsymbol{x}-\boldsymbol{y}|))\left[\tilde{b}_{f}(\boldsymbol{x}, \boldsymbol{y})-\tilde{b}_{g}(\boldsymbol{x}, \boldsymbol{y})\right] .
$$

We use the expansion (2.7) and equation (2.9), denoting $l$ by $l_{f}$ and $l_{g}$ to indicate its dependence on $f$ and $g$. We obtain

$$
\begin{aligned}
\tilde{b}_{\text {global }}(\boldsymbol{x}, \boldsymbol{y}) & =-\frac{i \kappa}{2 \pi} f(\boldsymbol{x}) \frac{e^{i \kappa|\boldsymbol{x}-\boldsymbol{y}|}}{1+|\boldsymbol{x}-\boldsymbol{y}|^{2}} f(\boldsymbol{y}) J_{f}(\boldsymbol{y})+l_{f}(\boldsymbol{x}, \boldsymbol{y}) \\
& -\left(-\frac{i \kappa}{2 \pi} g(\boldsymbol{x}) \frac{e^{i \kappa|\boldsymbol{x}-\boldsymbol{y}|}}{1+|\boldsymbol{x}-\boldsymbol{y}|^{2}} g(\boldsymbol{y}) J_{g}(\boldsymbol{y})+l_{g}(\boldsymbol{x}, \boldsymbol{y})\right),
\end{aligned}
$$

which we rewrite in the more convenient way

$$
\begin{aligned}
\tilde{b}_{\text {global }}(\boldsymbol{x}, \boldsymbol{y}) & =-\frac{i \kappa}{2 \pi} f(\boldsymbol{x}) \frac{e^{i \kappa|\boldsymbol{x}-\boldsymbol{y}|}}{1+|\boldsymbol{x}-\boldsymbol{y}|^{2}}(f(\boldsymbol{y})-g(\boldsymbol{y})) J_{f}(\boldsymbol{y}) \\
& -\frac{i \kappa}{2 \pi}(f(\boldsymbol{x})-g(\boldsymbol{x})) \frac{e^{i \kappa|\boldsymbol{x}-\boldsymbol{y}|}}{1+|\boldsymbol{x}-\boldsymbol{y}|^{2}} g(\boldsymbol{y}) J_{f}(\boldsymbol{y}) \\
& -\frac{i \kappa}{2 \pi} g(\boldsymbol{x}) \frac{e^{i \kappa|\boldsymbol{x}-\boldsymbol{y}|}}{1+|\boldsymbol{x}-\boldsymbol{y}|^{2}} g(\boldsymbol{y})\left(J_{f}(\boldsymbol{y})-J_{g}(\boldsymbol{y})\right) \\
& +l_{f}(\boldsymbol{x}, \boldsymbol{y})-l_{g}(\boldsymbol{x}, \boldsymbol{y}), \quad \boldsymbol{x}, \boldsymbol{y} \in \mathbb{R}^{2}
\end{aligned}
$$

The integral operators whose kernel are the first three terms of (2.23) can be bounded using LEMmA 1.33 and Lemma 1.46 and the estimate (2.8), noting that REMARK 2.5 guarantees the uniformity of (2.8) for $f \in B$. To bound the integral operator whose kernel is the last term of $(2.23)$, we construct, for every $\eta \in(0,1)$, a function $\ell_{\eta} \in L^{1}\left(\mathbb{R}^{2}\right)$ such that

$$
\left|l_{f}(\boldsymbol{x}, \boldsymbol{y})-l_{g}(\boldsymbol{x}, \boldsymbol{y})\right| \leq \ell_{\eta}(\boldsymbol{x}-\boldsymbol{y}), \quad \boldsymbol{x}, \boldsymbol{y} \in \mathbb{R}^{2},
$$

whenever $f, g \in B$ and $\|f-g\|_{B C^{1, \alpha}\left(\mathbb{R}^{2}\right)}$ is sufficiently small, and such that

$$
\left\|\ell_{\eta}\right\|_{L^{1}\left(\mathbb{R}^{2}\right)} \rightarrow 0, \quad \eta \rightarrow 0
$$

and then we use the estimate (1.54). Together, the bounds on the four parts of $\tilde{B}_{\text {global }}$ show (2.21) for the global part of the operator.

The construction of $\ell_{\eta}$ is as follows. We choose (possible by REMARK 2.5) a constant $C>0$ so that $(2.8)$ holds for all $f \in B$. Then, where $\tilde{\ell} \in L^{1}\left(\mathbb{R}^{2}\right)$ is defined as in LEMMA 2.3, we set

$$
\ell_{\eta}(\boldsymbol{y}):=\left\{\begin{array}{lc}
\eta^{3}, & \eta<|\boldsymbol{y}|<\eta^{-1} \\
2 C \tilde{\ell}(\boldsymbol{y}), & \text { otherwise }
\end{array}\right.
$$


Clearly this satisfies that $\left\|\ell_{\eta}\right\|_{L^{1}\left(\mathbb{R}^{2}\right)} \rightarrow 0$ as $\eta \rightarrow 0$. Since, for every $\eta \in(0,1)$,

$$
\left|l_{f}(\boldsymbol{x}, \boldsymbol{y})-l_{g}(\boldsymbol{x}, \boldsymbol{y})\right| \rightarrow 0, \quad\|f-g\|_{B C^{1, \alpha}\left(\mathbb{R}^{2}\right)} \rightarrow 0
$$

uniformly in $f$ and $g$ for $f, g \in B$, and uniformly in $\boldsymbol{x}$ and $\boldsymbol{y}$ for $\eta \leq|\boldsymbol{x}-\boldsymbol{y}| \leq \eta^{-1}$, the bound (2.24) holds for all $f, g \in B$ with $\|f-g\|_{B C^{1, \alpha}\left(\mathbb{R}^{2}\right)}$ sufficiently small.

The local operator. For the local operator we argue in a similar way as for the global operator, in particular in a similar way as for the integral operator corresponding to the last term in (2.23). In particular, where $\tilde{b}_{\text {local }}$ is the kernel of the local operator, it holds for every $\eta>0$ that $\left|\tilde{b}_{\text {local }}(\boldsymbol{x}, \boldsymbol{y})\right| \rightarrow 0$ as $\|f-g\|_{B C^{1, \alpha}\left(\mathbb{R}^{2}\right)} \rightarrow 0$, uniformly in $f$ and $g$ for $f, g \in B$, and uniformly in $\boldsymbol{x}$ and $\boldsymbol{y}$ for $|\boldsymbol{x}-\boldsymbol{y}| \geq \eta$, and (2.5) takes the role of (2.8).

To show later that the limiting absorption condition (8) is satisfied in the case $\kappa>0$ we need the following theorem.

Theorem 2.10. Denote $S$ and $K$ temporarily by $S_{\kappa_{i}}$ and $K_{\kappa_{i}}$ to indicate their dependence on $\kappa_{i}$. Then, where $B_{\kappa_{i}}$ denotes either $S_{\kappa_{i}}$ or $K_{\kappa_{i}}$, it holds that

$$
\left\|B_{\kappa_{i}}-B_{0}\right\|_{L^{2}(\Gamma) \rightarrow L^{2}(\Gamma)} \rightarrow 0
$$

as $\kappa_{i} \rightarrow 0$.

Proof. As we did when proving Theorem 2.6 we split $B_{\kappa_{i}}$ into global and local parts, as $B_{\kappa_{i}}=B_{1}+B_{2}$, with $B_{1}, B_{2}$ defined by (2.3) and (2.4). As in the proofs of LEMMA 2.1 and LemMA 2.3 we denote the kernel of $B_{j}$ by $b_{j}$.

To show (2.26) for the local part $B_{2}$ we note that $b_{2}(x, y)$ depends continuously on $\kappa_{i}$, uniformly in $x$ and $y$ for $|x-y| \geq \eta$ and every $\eta>0$, and that, by REMARK 2.5, the bound (2.5) holds uniformly in $\kappa_{i}$ for $\kappa_{i} \in[0,1]$. We then argue as for the local part in the proof of THEOREM 2.9, showing that the kernel of the local part of $B_{\kappa_{i}}-B_{0}$ is bounded by an $L^{1}$ convolution kernel $\ell(\boldsymbol{x}-\boldsymbol{y})$ with $\|\ell\|_{L^{1}\left(\mathbb{R}^{2}\right)} \rightarrow 0$ as $\kappa_{i} \rightarrow 0$. Finally we apply (1.54).

To show (2.26) for the global part $B_{2}$ we use the representation (2.7) for $b_{1}(x, y)$, which splits $b_{1}$ into a weakly singular part $l(\boldsymbol{x}, \boldsymbol{y})$, bounded by $(2.8)$, and a strongly singular part $l^{*}(\boldsymbol{x}, \boldsymbol{y})$, given explicitly by (2.9) or (2.10). To show (2.26) for the weakly singular part of $B_{2}$ we argue exactly as we did in the proof of THEOREM 2.9, noting that, by REMARK 2.5, (2.8) holds uniformly in $\kappa_{i}$ for $\kappa_{i} \in[0,1]$, and that $l(\boldsymbol{x}, \boldsymbol{y})$ depends continuously on $\kappa_{i}$, uniformly in $\boldsymbol{x}$ and $\boldsymbol{y}$ for $\eta \leq|\boldsymbol{x}-\boldsymbol{y}| \leq \eta^{-1}$, for every $\eta \in(0,1)$. That $(2.26)$ holds for the strongly singular part of $B_{2}$ follows from LEMMA 1.34 and (1.51). 


\section{Chapter 3}

\section{Existence and Uniqueness}

In this chapter we prove results on the equivalence of the boundary integral equation and the boundary value problem. We show that the boundary value problem has at most one solution and prove a result on the solvability of the integral equation, in a first step in the case of a flat surface and then in a second step for the general case of an arbitrary rough surface.

\subsection{Uniqueness}

Our first objective is to prove the following theorem on the equivalence of the integral equation and boundary value problem, using various results already shown above.

TheOrem 3.1 (Equivalence). Suppose that $v$ is defined by (15)-(17) with $\varphi \in X_{\infty}$. Then, in the case $\kappa_{i}>0, v$ satisfies the boundary value problem if and only if $\varphi$ satisfies the BIE (18). In the case $\kappa_{i}=0$ (i.e., $\kappa>0$ ), if $v$ satisfies the boundary value problem, then $\varphi$ satisfies (18). Conversely, if $\kappa>0, \varphi^{(\kappa+i \epsilon)} \in X_{\infty}$ satisfies the integral equation (18) with $\kappa$ replaced by $\kappa+i \epsilon$, for all sufficiently small $\epsilon>0$, and $\left\|\varphi-\varphi^{(\kappa+i \epsilon)}\right\|_{L_{2}(\Gamma)} \rightarrow 0$ as $\epsilon \rightarrow 0$, then $v$ satisfies the boundary value problem.

Proof. Let $v$ be the combined single- and double-layer potential $v$, defined in (15), with density $\varphi \in X_{\infty}$. By Theorem 2.8, $v \in C^{2}(D) \cap C(\bar{D})$ and satisfies the Helmholtz equation in $D$. Further, due to the jump relations (2.13) and (2.14), $v=g \in X_{\infty}$ on $\Gamma$ if and only if the density $\varphi$ satisfies the boundary integral equation (18). Applying TheOrem 2.8 again, we see that $v$ satisfies the bound (7). This yields the equivalence statement for $\kappa_{i}>0$.

For real $\kappa$, in addition, we need to show the limiting absorption principle (8). Let $a(x, y)=\partial G(x, y) / \partial \nu(y)-i \eta G(x, y)$, so that

$$
v(x)=\int_{\Gamma} a(x, y) \varphi(y) d s(y), \quad x \in D .
$$

Suppose, as stated in the theorem, that $\varphi^{(\kappa+i \epsilon)} \in X_{\infty}$ satisfies the integral equation (18) with $\kappa$ replaced by $\kappa+i \epsilon$, for all sufficiently small $\epsilon>0$, and that $\| \varphi-$ 
$\varphi^{(\kappa+i \epsilon)} \|_{L_{2}(\Gamma)} \rightarrow 0$ as $\epsilon \rightarrow 0$. Let $a^{(\kappa+i \epsilon)}$ denote $a$ with $\kappa$ replaced with $\kappa+i \epsilon$ and define $v^{(\kappa+i \epsilon)}$ by $(3.1)$ with $a, \varphi$, replaced by $a^{(\kappa+i \epsilon)}, \varphi^{(\kappa+i \epsilon)}$, respectively. We have shown in the previous chapter that $v^{(\kappa+i \epsilon)}$ satisfies PROBLEM 2 (with $\kappa$ replaced by $\kappa+i \epsilon)$. To show the limiting absorption principle (8) we need to show that $v^{(\kappa+i \epsilon)}(x) \rightarrow v(x)$ as $\epsilon \rightarrow 0$. We have

$$
\begin{aligned}
v^{(\kappa+i \epsilon)}(x)-v(x) & =\int_{\Gamma}\left(a^{(\kappa+i \epsilon)}(x, y)-a(x, y)\right) \varphi^{(\kappa+i \epsilon)}(y) d s(y) \\
& +\int_{\Gamma} a(x, y)\left(\varphi^{(\kappa+i \epsilon)}(y)-\varphi(y)\right) d s(y) .
\end{aligned}
$$

We see that the second term tends to zero as $\epsilon \rightarrow 0$ by the bound (2.16). Clearly, $a^{(\kappa+i \epsilon)}(x, y)-a(x, y) \rightarrow 0$ as $\epsilon \rightarrow 0$, for every $y \in \Gamma$. Thus, applying Cauchy-Schwarz and then the dominated convergence theorem, noting that the bound (2.19) holds uniformly in $\kappa$, we see that the first term tends to 0 as $\epsilon \rightarrow 0$.

We now prove the following uniqueness result.

TheOrem 3.2 (Uniqueness). The boundary value problem has at most one solution.

Proof. Due to [15, Theorem 1], see also [50, Theorem 3.1], a solution $u \in C^{2}(G) \cap$ $C(\bar{G})$ to the Helmholtz equation (4) with $\Im(\kappa)>0$ on an open set $G \subset \mathbb{R}^{n}$ which satisfies the growth condition $|u(x)| \leq C e^{\theta|x|}$, with some constant $\theta<\Im(\kappa)$, and the boundary condition $u(x)=0$ for $x \in \partial G$ will vanish identically on $G$. This result directly implies uniqueness for the scattering problem and the boundary value problem for $\kappa_{i}>0$. For $\kappa_{i}=0$ uniqueness is a consequence of the limiting absorption principle we require, i.e. of the convergence (8).

Before we turn to establishing existence of solutions, we show that to establish unique solvability of the integral equation in the space $X(\Gamma)$ or $X_{\infty}(\Gamma)$, it is enough to study the solvability in $L^{2}(\Gamma)$.

THEOREM 3.3. If the integral operator A, given through (19), is invertible in $B L\left(L^{2}(\Gamma)\right)$, the Banach algebra of bounded linear operators on $L^{2}(\Gamma)$, then it is also invertible in the subalgebras $B L(X(\Gamma))$ and $B L\left(X_{\infty}(\Gamma)\right)$.

Proof. We first note that the second statement can be proven in complete analogy to the first.

Assume that $A$ is invertible in $B L\left(L^{2}(\Gamma)\right)$, i.e. the integral equation (18) has exactly one solution $\psi \in L^{2}(\Gamma)$ for every $g \in X(\Gamma) \subset L^{2}(\Gamma)$. Then, defining $B=K-i \eta S$, it holds that $\psi=B \psi+2 g$ and, by induction, that, for every $n \in \mathbb{N}$,

$$
\psi=B^{n} \psi+2\left(B^{n-1}+\cdots+B^{0}\right) g .
$$


Now, by Theorem 2.6, $B$ is a bounded operator on $X(\Gamma)$ and, by Corollary 2.7, $B^{n}$ is a bounded operator from $L^{2}(\Gamma)$ to $X(\Gamma)$, for some $n \in \mathbb{N}$. Thus $\psi \in X(\Gamma)$. We have shown that (18) has exactly one solution $\psi \in X(\Gamma)$ for every $g \in X(\Gamma)$, so that $(I+K-i \eta S)^{-1}$ exists as an operator on $X(\Gamma)$. Since $X(\Gamma)$ is a Banach space it follows as a standard corollary of the open mapping theorem that $(I+K-i \eta S)^{-1}$ is bounded and thus $A$ is invertible in $B L(X(\Gamma))$.

As a corollary of Theorem 3.1, Theorem 3.2, TheOrem 2.10 and TheOrem 3.3 we have the following result:

Corollary 3.4. If $(I+K-i \eta S)^{-1}$ exists as a bounded operator on $L^{2}(\Gamma)$, then the boundary value problem and scattering problem have exactly one solution.

Proof. In the case $\kappa_{i}>0$ this result is clear from TheOrem 3.1 and TheOrem 3.2 and THEOREM 3.3.

In the case $\kappa_{i}=0$ we note that, by THEOREM 2.10 and standard operator perturbation arguments (e.g. [49]), if $(I+K-i \eta S)^{-1}$ exists as a bounded operator on $L^{2}(\Gamma)$ for $\kappa=\kappa_{0}>0$, then $(I+K-i \eta S)^{-1}$ exists and is a bounded operator on $L^{2}(\Gamma)$ for $\kappa=\kappa_{0}+i \kappa_{i}, 0 \leq \kappa_{i} \leq c$, for some $c>0$. Moreover, $(I+K-i \eta S)^{-1}$ depends continuously in the norm topology on $\kappa_{i}$, for $\kappa_{i} \in[0, c]$. Thus, provided $g \in L^{2}(\Gamma)$ depends continuously in norm on $\kappa_{i}$, for $\kappa_{i} \in[0, c]$, it holds that $(I+K-i \eta S)^{-1} g$ depends continuously in norm on $\kappa_{i} \in[0, c]$, in $L^{2}(\Gamma)$. If $g$ is given by (14), then, from the continuity of $\Phi(x, y)$ as a function of $\kappa_{i}$, uniformly in $x, y \in \mathbb{R}^{3}, x \neq y$, the bound (1.22), and the dominated convergence theorem, it follows that $g \in L^{2}(\Gamma)$ depends continuously in norm on $\kappa_{i}$, for $\kappa_{i} \in[0, c]$. Thus the result follows by Theorem 3.1, Theorem 3.2 and Theorem 3.3.

Combining Theorem 3.3 and Corollary 3.4 yields the next theorem.

THEOREM 3.5. If $A$ is invertible as an operator on $L^{2}(\Gamma)$, then $A$ is invertible as an operator on $X_{\infty}$. Moreover, if $A$ is invertible on $X_{\infty}$, then the boundary value problem has exactly one solution $v$, defined by (15)-(17) with $\varphi \in X_{\infty}$ given by $\varphi=2 A^{-1} g$. Further, for some constant $c>0$, independent of $g$,

$$
|v(x)| \leq c\|g\|_{X_{\infty}}, \quad x \in \bar{D} .
$$

\subsection{Invertibility of $I+K-i \eta S$}

THEOREM 3.5 shows that it suffices to prove the invertibility of $A$ to answer the solvability and existence of solutions to the boundary value problem and the original scattering problem. We address this remaining task in this section. 
We start with our investigation on the invertibility of the operator $A$ in the simple case of a flat scattering surface $\Gamma^{h}$, extending this result in a second step to the general case of a rough surface.

\subsubsection{The case of a flat surface}

In the case that the scattering surface is given in form of a flat surface

$$
\Gamma^{h}=\left\{y=(\boldsymbol{y}, h): \boldsymbol{y} \in \mathbb{R}^{2}\right\}, \quad h>0,
$$

we see that the kernels of $K$ and $S$ only depend on the difference $\boldsymbol{x}-\boldsymbol{y}$ and thus, identifying $\Gamma^{h}$ with $\mathbb{R}^{2}$, the operators are convolution operators on $L^{2}\left(\mathbb{R}^{2}\right)$.

As we have already discussed at the end of Section 1.5.2 the kernel of the singleand double-layer operators can be expressed with the help of the function $W_{h}$ defined by (1.38). Hence, the integral equation (18) can be written as

$$
\psi(\boldsymbol{x})+\int_{\mathbb{R}^{2}}\left\{P_{h}(\boldsymbol{x}-\boldsymbol{y})-i \eta Q_{h}(\boldsymbol{x}-\boldsymbol{y})\right\} \psi(\boldsymbol{y}) d \boldsymbol{y}=2 g(\boldsymbol{x}), \quad \boldsymbol{x} \in \mathbb{R}^{2},
$$

where $Q_{h}$ and $P_{h}$ are given through (1.41) and (1.42). Introducing the function

$$
R_{h}:=P_{h}-i \eta Q_{h}
$$

we can write (3.2) shortly as

$$
\psi+R_{h} * \psi=2 g .
$$

We will show that this equation is uniquely solvable by means of Fourier transformation. To apply the convolution theorem for $L^{2}$-functions (THEOREM 1.43), we need to show first that $R_{h} * \psi \in L^{2}\left(\mathbb{R}^{2}\right)$ for all $\psi \in L^{2}\left(\mathbb{R}^{2}\right)$. This is of course a consequence of the mapping properties we showed for the double- and single-layer potential operator, cf. THEOREM 2.6.

REMARK 3.6. In the case of a flat scattering surface the mapping properties of $S$ and $K$ are a direct consequence of LEMMA 1.44. It follows from LEMMA 1.37 and LEMMA 1.38 that $\mathcal{F}_{2} R_{h} \in L^{2}\left(\mathbb{R}^{2}\right) \cap L^{\infty}\left(\mathbb{R}^{2}\right)$, so we can apply LEMMA 1.44 to conclude that $R_{h} * \psi \in L^{2}\left(\mathbb{R}^{2}\right)$ for all $\psi \in L^{2}\left(\mathbb{R}^{2}\right)$, i.e. the boundedness of $\mathcal{F}_{2} R_{h}$ ensures that $R_{h} * \psi \in L^{2}\left(\mathbb{R}^{2}\right)$.

Combining the Fourier transforms of $P_{h}$ and $Q_{h}$ that we computed in LEMMA 1.35, we derive for the Fourier transform of $R_{h}$ the formula $\left(\mathcal{F}_{2} R_{h}\right)(\boldsymbol{k})=\hat{r}_{h}(|\boldsymbol{k}|)$, for almost all $\boldsymbol{k} \in \mathbb{R}^{2}$, where

$$
\hat{r}_{h}(k):=\frac{1}{2 \pi}\left\{e^{-2 h \sqrt{k^{2}-\kappa^{2}}}-i \eta \frac{1-e^{-2 h \sqrt{k^{2}-\kappa^{2}}}}{\sqrt{k^{2}-\kappa^{2}}}\right\}, \quad k \geq 0 .
$$


From the convolution theorem for $L^{2}$-functions (THEOREM 1.43) we see that, for $\psi \in L^{2}\left(\mathbb{R}^{2}\right)$,

$$
\begin{aligned}
(I+K-i \eta S) \psi & =\psi+R_{h} * \psi \\
& =\mathcal{F}_{2}^{-1}\left(\left(1+2 \pi \mathcal{F}_{2} R_{h}\right) \cdot\left(\mathcal{F}_{2} \psi\right)\right) .
\end{aligned}
$$

Since $\mathcal{F}_{2}$ is an isomorphism on $L^{2}\left(\mathbb{R}^{2}\right)$ it follows that the inverse of $I+K-i \eta S$ exists as a bounded operator from $L^{2}\left(\Gamma^{h}\right)$ into $L^{2}\left(\Gamma^{h}\right)$ if and only if

$$
\underset{\boldsymbol{k} \in \mathbb{R}^{2}}{\text { ess. }} \inf \left|1+2 \pi\left(\mathcal{F}_{2} R_{h}\right)(\boldsymbol{k})\right|=\inf _{k \geq 0}\left|1+2 \pi \hat{r}_{h}(k)\right|>0 .
$$

Thus we need to investigate

$$
K(k):=1+2 \pi \hat{r}_{h}(k)=A\left(h \sqrt{k^{2}-\kappa^{2}}\right), \quad k \geq 0,
$$

where

$$
A(z):=1+e^{-2 z}-\frac{i h \eta}{z}\left(1-e^{-2 z}\right) .
$$

We recall that the square root is to be taken with $\sqrt{k^{2}-\kappa^{2}} \in V:=\{z \in \mathbb{C}: \Re z \geq$ $0, \Im z \leq 0\}$, cf. (1.39). Indeed, in the case that $\kappa_{i}>0$, so that $\Im\left(k^{2}-\kappa^{2}\right)<0$, it is clear that $\sqrt{k^{2}-\kappa^{2}}$ lies in the interior of $V$. Now $A$ is an entire function (the singularity at 0 is removable) so that $K$ is continuous on $[0, \infty)$. Further, $K(k) \rightarrow 1$ as $k \rightarrow \infty$. Thus, to show (3.5) it is enough to show that $K(k) \neq 0$ for $k \geq 0$ which holds if $A(z) \neq 0$ for $z \in V$; indeed, in the case $\kappa_{i}>0$, we need only show that $A(z) \neq 0$ for all $z$ in the interior of $V$.

So suppose $\eta \geq 0$ and consider first the case when $z=z_{r}-i z_{i}$, with $z_{r}>0$, $z_{i} \geq 0$. It holds that

$$
A(z)=-i\left(1+e^{-2 z}\right)\left(\frac{h \eta \tanh z}{z}+i\right)
$$

and straightforward calculations yield

$$
\Im\left(\frac{\tanh z}{z}\right)=\frac{z_{r} \sin \left(2 z_{i}\right)+z_{i} \sinh \left(2 z_{r}\right)}{2\left[\sinh ^{2} z_{r}+\cos ^{2} z_{i}\right]\left(z_{r}^{2}+z_{i}^{2}\right)} \geq 0
$$

since $|\sin t| \leq t \leq \sinh t$ for $t \geq 0$. Thus (3.5) holds if $\eta \geq 0$ and $\kappa_{i}>0$.

In the case $\kappa_{i}=0$ we need to show, additionally, that $A(z) \neq 0$ when $z=-i z_{i}$ with $z_{i} \geq 0$, in order to establish that $A(z) \neq 0$ for all $z \in V$. Now $A(0)=2-2 i \eta h$ and, for $z_{i}>0$, from (3.6),

$$
A\left(-i z_{i}\right)=2 \cos z_{i}-\frac{2 i h \eta}{z_{i}} \sin z_{i}
$$


Thus, provided $\eta>0, A\left(-i z_{i}\right) \neq 0$ for $z_{i} \geq 0$ so $A(z) \neq 0$ for $z \in V$. Thus (3.5) holds if $\eta>0$.

We have proven, in the case $\eta>0$ and in the case $\eta=0, \kappa_{i}>0$, that (3.5) holds, and thus we have shown the invertibility of $I+K-i \eta S$ and the boundedness of the inverse operator in $L^{2}\left(\Gamma^{h}\right)$. Thus we have established the solvability of (18) in the space $L^{2}(\Gamma)$ for flat surfaces, which we summarise in the next lemma.

Lemma 3.7. In the case $\Gamma=\Gamma^{h}$, with $h>0$ it holds that $A$ is invertible as an operator on $L^{2}(\Gamma)$.

In the case that $\Gamma_{f}$ is mildly rough, i.e. $\Gamma_{f}$ does not differ too much from a flat surface $\Gamma^{h}$, we can use THEOREM 2.9 to show that the integral equation remains solvable. We state this intermediate result in the following lemma.

Lemma 3.8. Suppose that $h>0$ and that either $\eta>0$ or $\eta=0$ and $\kappa_{i}>0$. Then, provided $\|f-h\|_{B C^{1, \alpha}\left(\mathbb{R}^{2}\right)}$ is sufficiently small it holds that $(I+K-i \eta S)^{-1}$ exists and is bounded as an operator on $L^{2}\left(\Gamma_{f}\right)$.

Proof. Let $A=I+K-i \eta S$, and then denote $A$ by $A_{f}$ to denote its dependence on $f$. With the help of the isomorphism $I_{f}: L^{2}\left(\Gamma_{f}\right) \rightarrow L^{2}\left(\mathbb{R}^{2}\right)$ defined by (1.9) we associate $A_{f}$ with the element $\tilde{A}_{f}=I_{f} A_{f} I_{f}^{-1}$ of the set of bounded linear operators on $L^{2}\left(\mathbb{R}^{2}\right)$. Now, $\tilde{A}_{h}$ is invertible with bounded inverse, by our analysis above for the flat plane case. Moreover, by the continuity of $\tilde{A}_{f}$ with respect to $f$ as proven in Theorem 2.9 it follows from standard arguments that $\tilde{A}_{f}$ is boundedly invertible on $L^{2}\left(\mathbb{R}^{2}\right)$ for $\|f-h\|_{B C^{1, \alpha}\left(\mathbb{R}^{2}\right)}$ sufficiently small and so $A_{f}$ is boundedly invertible on $L^{2}\left(\Gamma_{f}\right)$.

\subsubsection{The case of a general rough surface}

In this last section we briefly summarise the essential steps that are needed to show the invertibility of the operator $A$ for the case of a general rough surface. The results have appeared in [22] and we refer the reader to the article for the details of the proofs. The key ingredients that are needed for the proofs are THEOREM 2.6, THEOREM 3.5, LEMmA 3.7 and ThEOREM 2.9, certain results from [14], and standard properties of layer potentials.

Recall the definition of the dual-pairing

$$
\langle\phi, \psi\rangle=\int_{\Gamma} \phi(y) \psi(y) d s(y), \quad \phi, \psi \in L^{2}(\Gamma),
$$

given in Section 1.1.5. With respect to this dual-pairing we introduce the operator $K^{\prime}$ defined by

$$
\left(K^{\prime} \varphi\right)(x):=2 \int_{\Gamma} \frac{\partial G(x, y)}{\partial \nu(x)} \varphi(y) d s(y), \quad x \in \Gamma
$$


which is the adjoint of the double-layer operator $K$. In complete analogy to the case of the single- and double-layer operator, cf. THEOREM 2.6, one proves the following lemma.

LEMMA 3.9. $K^{\prime}$ is a bounded operator on $L^{2}(\Gamma)$.

The operators $S$ and $I$ are both self-adjoint with respect to the dual-pairing so that the adjoint of $A$ is

$$
A^{\prime}=I+K^{\prime}-i \eta S .
$$

We recall the following standard properties of adjoint operators on Hilbert spaces, cf. Section 1.1.5 and the citations therein. We have that $A$ and $A^{\prime}$ have the same norm, that $A$ is invertible if and only if $A^{\prime}$ is invertible, and that if they are both invertible then

$$
\left\|A^{-1}\right\|_{L^{2}(\Gamma) \rightarrow L^{2}(\Gamma)}=\left\|A^{\prime-1}\right\|_{L^{2}(\Gamma) \rightarrow L^{2}(\Gamma)} .
$$

Thus, we can proceed in the first instance by bounding $A^{\prime}$. The first step in this direction is to prove the following lower bound in the case when $\Gamma$ is smooth.

LEMMA 3.10. Suppose that, in addition to our assumptions throughout on $f$, it holds that $f \in C^{\infty}\left(\mathbb{R}^{2}\right)$. Then, for all $\varphi \in L^{2}(\Gamma)$ there holds

$$
\left\|A^{\prime} \varphi \mid\right\|_{L^{2}(\Gamma)} \geq B^{-1}\|\varphi\|_{L^{2}(\Gamma)}
$$

where

$$
B=B\left(L_{f}, \kappa / \eta\right):=\frac{1}{2}\left(1+\left(\frac{3 \kappa^{2} \tilde{L}}{\eta^{2}}\left[5 \tilde{L}+6 L_{f}^{2}\right]+6\left(\tilde{L}+3 L_{f}^{2}\right)^{2}\right)^{1 / 2}\right)
$$

and $\tilde{L}:=\left(1+L_{f}^{2}\right)^{1 / 2}$ and $L_{f}$ denotes the maximum surface slope of $\Gamma_{f}$.

Proof. For a proof see [22, Lemma 3.3].

The previous lemma is the main tool to prove the general result, namely the following theorem.

Theorem 3.11. A and $A^{\prime}$ are invertible on $L^{2}\left(\Gamma_{f}\right)$ for any $f \in B C^{1, \alpha}\left(\mathbb{R}^{2}\right)$, with

$$
\left\|A^{-1}\right\|_{L^{2}\left(\Gamma_{f}\right) \rightarrow L^{2}\left(\Gamma_{f}\right)}=\left\|A^{\prime-1}\right\|_{L^{2}\left(\Gamma_{f}\right) \rightarrow L^{2}\left(\Gamma_{f}\right)} \leq B
$$

where $B=B\left(L_{f}, \kappa / \eta\right)$ is defined by (3.10).

Proof. For a proof see [22, Theorem 3.4].

Combining the result of the last theorem together with THEOREM 3.5 yields the final result. 
THEOREM 3.12. A is invertible as an operator on $L^{2}(\Gamma)$ and as an operator on $X_{\infty}$. Moreover, the BVP has exactly one solution $v$, defined by (15)-(17) with $\varphi \in X_{\infty}$ given by $\varphi=2 A^{-1} \mathrm{~g}$. Further, for some constant $c>0$, independent of $g$,

$$
|v(x)| \leq c\|g\|_{X_{\infty}}, \quad x \in \bar{D} .
$$


Part II

Numerics 



\section{Chapter 4}

\section{Tools}

In this chapter we introduce notations, summarise results from multi-dimensional Fourier analysis and prove error estimates for the composite trapezoidal rule on weighted spaces of differentiable functions.

\subsection{Notations}

The components of a vector $x \in \mathbb{R}^{d}$ for some $d \in \mathbb{N}$ are denoted by $\left(x_{1}, \ldots, x_{d}\right)$. The standard scalar product of two vectors $x, y \in \mathbb{R}^{d}$ is denoted by $\langle x, y\rangle$. The standard basis vectors are denoted by $e_{j}$ for $j=1, \ldots, d$. For two vectors $x, y \in \mathbb{R}^{d}$ we define

$$
x<y \quad: \Longleftrightarrow x_{j}<y_{j} \quad \text { for } \quad j=1, \ldots, d
$$

and

$$
x \leq y \quad: \Longleftrightarrow x_{j} \leq y_{j} \quad \text { for } \quad j=1, \ldots, d,
$$

thus extending the definition for multi-indices to elements in $\mathbb{R}^{d}$, cf. Definition 1.2. Let $x, y \in \mathbb{R}^{d}$ with $x<y$, then we write

$$
[x, y]:=\times_{j=1}^{d}\left[x_{j}, y_{j}\right]:=\left[x_{1}, y_{1}\right] \times \cdots \times\left[x_{d}, y_{d}\right] \subset \mathbb{R}^{d} .
$$

for the generalised interval in $\mathbb{R}^{d}$. For $x \in \mathbb{R}_{>0}^{d}$, i.e. $0<x$, we write

$$
\sharp(x):=\prod_{j=1}^{d} x_{j}
$$

for the product of the components, hence the volume of the generalised interval $[x, y]$ is given through $\sharp(y-x)$.

A pointwise multiplication and division is defined through

$$
x \odot y:=\left(x_{1} y_{1}, \ldots, x_{d} y_{d}\right) \in \mathbb{R}^{d}
$$


and

$$
x \div y:=\left(\frac{x_{1}}{y_{1}}, \ldots, \frac{x_{d}}{y_{d}}\right) \in \mathbb{R}^{d}
$$

if $y_{j} \neq 0$ for $j=1, \ldots, d$.

Let $N, M \in \mathbb{Z}^{d}$ with $N<M$. We call a function $a:[N, M] \subset \mathbb{Z}^{d} \rightarrow \mathbb{C}$ a $d$-dimensional array. We write this as

$$
a=\left(a_{j_{1}, \ldots, j_{d}}\right)_{j_{1}=N_{1}, \ldots, M_{1} ; \ldots ; j_{d}=N_{d}, \ldots, M_{d}} \in \mathbb{C}^{\left(M_{1}-N_{1}+1\right) \times \cdots \times\left(M_{d}-N_{d}+1\right)} .
$$

As short form we abbreviate this by

$$
a=\left(a_{j}\right)_{j=N, \ldots, M} \in \mathbb{C}^{\times(M-N+1)},
$$

where we used the convention

$$
j=N, \ldots, M \quad: \Longleftrightarrow \quad j_{i}=N_{i}, \ldots, M_{i} \quad \text { for } \quad i=1, \ldots, d,
$$

which can also be written as $N \leq j \leq M$. We write

$$
\sum_{M \leq j \leq N} a_{j}:=\sum_{j=N}^{M} a_{j}:=\sum_{j_{1}=N_{1}}^{M_{1}} \cdots \sum_{j_{d}=N_{d}}^{M_{d}} a_{j_{1}, \ldots, j_{d}}
$$

for the multiple sum.

A mapping $B:[N, M] \times[N, M] \subset \mathbb{Z}^{d} \times \mathbb{Z}^{d} \rightarrow \mathbb{C}$ is called a $d$-dimensional matrix and we write this as

$$
B=\left(b_{i, j}\right)_{i, j=N, \ldots, M} \in \mathbb{C}^{[\times(M-N+1)] \times[\times(M-N+1)]} .
$$

The matrix-vector product is defined in the usual way through

$$
(B \cdot a)_{i}=\sum_{j=N}^{M} b_{i, j} a_{j}, \quad i=N, \ldots, M .
$$

The $d$-dimensional matrices and arrays are closely related to $d$-level block matrices and ordinary column vectors. In fact, for any $d$-dimensional array $a=\left(a_{j}\right)_{j=N, \ldots, M} \in$ $\mathbb{C}^{\times(M-N+1)}$ we associate a uniquely determined vector $\tilde{a} \in \mathbb{C}^{\sharp(M-N+1) \times 1}$ with

$$
\tilde{a}=\left(a_{\left(N_{1}, \ldots, N_{d}\right)}, a_{\left(N_{1}, \ldots, N_{d}+1\right)}, \ldots, a_{\left(N_{1}, \ldots, M_{d}\right)}, \ldots, a_{\left(M_{1}, \ldots, M_{d}\right)}\right)^{t} .
$$

EXAMPLE 4.1. In the case $N=(1,2,3)$ and $M=(2,3,4)$ the vector is given through

$$
\tilde{a}=\left(a_{(1,2,3)}, a_{(1,2,4)}, a_{(1,3,3)}, a_{(1,3,4)}, a_{(2,2,3)}, a_{(2,2,4)}, a_{(2,3,3)}, a_{(2,3,4)}\right)^{t} .
$$


If we use this numbering, we can associate for each $d$-dimensional matrix $B=$ $\left(b_{i, j}\right)_{i, j=N, \ldots, M} \in \mathbb{C}^{[\times(M-N+1)] \times[\times(M-N+1)]}$ a matrix $\tilde{B} \in \mathbb{C}^{\sharp(M-N+1) \times \sharp(M-N+1)}$ that is given through the following set of rules:

The 0-level:

$$
\tilde{B}=B^{(0)}:=\left(B_{i_{1}, j_{1}}^{(1)}\right)_{i_{1}, j_{1}=N_{1}, \ldots, M_{1}},
$$

the 1-level:

$$
B_{i_{1}, j_{1}}^{(1)}:=\left({ }_{\left(i_{1}\right),\left(j_{1}\right)} B_{i_{1}, j_{1}}^{(2)}\right)_{i_{2}, j_{2}=N_{2}, \ldots, M_{2}},
$$

the $n$-level for $n=2, \ldots, d-1$ :

$$
\left(i_{1}, \ldots, i_{n-1}\right)\left(j_{1}, \ldots, j_{n-1}\right) B_{i_{n}, j_{n}}^{(n)}:=\left({ }_{\left(i_{1}, \ldots, i_{n}\right),\left(j_{1}, \ldots, j_{n}\right)} B_{i_{n+1}, j_{n+1}}^{(n+1)}\right)_{i_{n+1}, j_{n+1}=N_{n+1}, \ldots, M_{n+1}},
$$

the $d$-level:

$$
\left(i_{1}, \ldots, i_{d-1}\right)\left(j_{1}, \ldots, j_{d-1}\right) B_{i_{n}, j_{n}}^{(d)}:=b_{i, j} .
$$

\begin{tabular}{|c|c|c|c|c|c|c|c|}
\hline$b_{(1,2,3),(1,2,3)}$ & $b_{(1,2,3),(1,2,4)}$ & $b_{(1,2,3),(1,3,3)}$ & $b_{(1,2,3),(1,3,4)}$ & $b_{(1,2,3),(2,2,3)}$ & $b_{(1,2,3),(2,2,4)}$ & $b_{(1,2,3),(2,3,3)}$ & $b_{(1,2,3),(2,3,4)}$ \\
\hline$b_{(1,2,4),(1,2,3)}$ & $b_{(1,2,4),(1,2,4)}$ & $b_{(1,2,4),(1,3,3)}$ & $b_{(1,2,4),(1,3,4)}$ & $b_{(1,2,4),(2,2,3)}$ & $b_{(1,2,4),(2,2,4)}$ & $b_{(1,2,4),(2,3,3)}$ & $b_{(1,2,4),(2,3,4)}$ \\
\hline$b_{(1,3,3),(1,2,3)}$ & $b_{(1,3,3),(1,2,4)}$ & $b_{(1,3,3),(1,3,3)}$ & $b_{(1,3,3),(1,3,4)}$ & $b_{(1,3,3),(2,2,3)}$ & $b_{(1,3,3),(2,2,4)}$ & & $b_{(1,3,3),(2,3,4)}$ \\
\hline$b_{(1,3,4),(1,2,3)}$ & $b_{(1,3,4),(1,2,4)}$ & $b_{(1,3,4),(1,3,3)}$ & $b_{(1,3,4),(1,3,4)}$ & $b_{(1,3,4),(2,2,3)}$ & $b_{(1,3,4),(2,2,4)}$ & $b_{(1,3,4),(2,3,3)}$ & $b_{(1,3,4),(2,3,4)}$ \\
\hline$b_{(2,2,3),(1,2,3)}$ & $b_{(2,2,3),(1,2,4)}$ & $b_{(2,2,3),(1,3,3)}$ & $b_{(2,2,3),(1,3,4)}$ & $b_{(2,2,3),(2,2,3)}$ & $b_{(2,2,3),(2,2,4)}$ & $b_{(2,2,3),(2,3,3)}$ & $b_{(2,2,3),(2,3,4)}$ \\
\hline$b_{(2,2,4),(1,2,3)}$ & $b_{(2,2,4),(1,2,4)}$ & $b_{(2,2,4),(1,3,3)}$ & $b_{(2,2,4),(1,3,4)}$ & $b_{(2,2,4),(2,2,3)}$ & $b_{(2,2,4),(2,2,4)}$ & $b_{(2,2,4),(2,3,3)}$ & $b_{(2,2,4),(2,3,4)}$ \\
\hline, 3$),(1,2,3)$ & $2,4)$ & $, 3),(1,3,3)$ & $, 3,4)$ & $3),(2,2,3)$ & $3),(2,2,4)$ & $3,3),(2,3,3)$ & $b_{(2,3,3),(2,3,4)}$ \\
\hline$b_{(2,3,4),(1,2,3)}$ & $b_{(2,3,4),(1,2,4)}$ & $b_{(2,3,4),(1,3,3)}$ & $b_{(2,3,4),(1,3,4)}$ & $b_{(2,3,4),(2,2,3)}$ & $b_{(2,3,4),(2,2,4)}$ & $b_{(2,3,4),(2,3,3)}$ & $b_{(2,3,4),(2,3,4)}$ \\
\hline
\end{tabular}

EXAMPLE 4.2. We illustrate the above definitions for the case of a 3-level block matrix given through $N=(1,2,3)$ and $M=(2,3,4)$. The matrix $\tilde{B}$ is given through

The structure of the different levels is given through

$$
B^{(0)}=\left(\begin{array}{cc}
B_{1,1}^{(1)} & B_{1,2}^{(1)} \\
B_{2,1}^{(1)} & B_{2,2}^{(1)}
\end{array}\right)=\left(\begin{array}{rr|rr}
(1),(1) B_{2,2}^{(2)} & (1),(1) B_{2,3}^{(2)} & { }_{(1),(2)} B_{2,2}^{(2)} & { }_{(1),(2)} B_{2,3}^{(2)} \\
{ }_{(1),(1)} B_{3,2}^{(2)} & (1),(1) B_{3,3}^{(2)} & { }_{(1),(2)} B_{3,2}^{(2)} & { }_{(1),(2)} B_{3,3}^{(2)} \\
\hline{ }_{(2),(1)} B_{2,2}^{(2)} & { }_{(2),(1)} B_{2,3}^{(2)} & { }_{(2),(2)} B_{2,2}^{(2)} & { }_{(2),(2)} B_{2,3}^{(2)} \\
{ }_{(2),(1)} B_{3,2}^{(2)} & (2),(1) B_{3,3}^{(2)} & { }_{(2),(2)} B_{3,2}^{(2)} & (2),(2) B_{3,3}^{(2)}
\end{array}\right) .
$$




\subsection{Multi-dimensional Fourier analysis}

In this section we summarise some results from the theory of multi-dimensional Fourier analysis.

A function $f: \mathbb{R}^{d} \rightarrow \mathbb{C}$ is said to be multi-periodic with period vector $p$, if there exists a vector $p \in \mathbb{R}_{>0}^{d}$ such that

$$
f(x)=f\left(x+p_{j} e_{j}\right), \quad j=1, \ldots, d
$$

for all $x \in \mathbb{R}^{d}$. Such a function is completely determined by its values on the cube $[0, p]$.

The linear space of all complex valued, Lebesgue measurable functions on $\mathbb{R}^{d}$ that are multi-periodic with period vector $p \in \mathbb{R}_{>0}^{d}$ and for which

$$
\int_{[0, p]}|\varphi(x)|^{2} d x<\infty
$$

is denoted by $L_{p}^{2}\left(\mathbb{R}^{d}\right)$. As a shorthand notation we use $L_{p}^{2}$ synonymously. A scalarproduct is defined on $L_{p}^{2}$ through

$$
(\varphi, \psi)_{L_{p}^{2}}:=\frac{1}{\sharp(p)} \int_{[0, p]} \varphi(x) \overline{\psi(x)} d x .
$$

The linear space $L_{p}^{2}$ is a Hilbert space equipped with the norm

$$
\|\varphi\|_{L_{p}^{2}}:=\sqrt{(\varphi, \varphi)_{L_{p}^{2}}}, \quad \varphi \in L_{p}^{2}
$$

We denote by

$$
E_{p, n}(x):=e^{2 \pi i\langle n, x \div p\rangle}, \quad n \in \mathbb{Z}^{d}, x \in \mathbb{R}^{d},
$$

where $i$ denotes the imaginary unit. One easily checks the orthonormality property

$$
\left(E_{p, n}, E_{p, m}\right)_{L_{p}^{2}}= \begin{cases}1, & n=m, \\ 0, & \text { otherwise. }\end{cases}
$$

A function $a: \mathbb{Z}^{d} \rightarrow \mathbb{C}$ can be interpreted as a multivariate complex sequence $\left(a_{n}\right):=\left(a_{n}\right)_{n \in \mathbb{Z}^{d}}$ where $a_{n}:=a(n)$ for $n \in \mathbb{Z}^{d}$. In a straightforward manner we define the multivariate series of $\left(a_{n}\right)_{n \in \mathbb{Z}^{d}}$ as the limit of the $m$-th partial sum $s_{m}(a)$, i.e.

$$
\sum_{n \in \mathbb{Z}^{d}} a_{n}:=\lim _{m \rightarrow \infty} s_{m}(a):=\lim _{m \rightarrow \infty} \sum_{\substack{n \in \mathbb{Z}^{d} \\\|n\|_{1} \leq m}} a_{n},
$$

where $\|n\|_{1}=\sum_{j=1}^{d}\left|n_{j}\right|$ for $n=\left(n_{1}, \ldots, n_{d}\right) \in \mathbb{Z}^{d}$. The linear space of all square summable multivariate complex valued sequences is denoted by

$$
\ell^{2}\left(\mathbb{Z}^{d}, \mathbb{C}\right):=\ell^{2}\left(\mathbb{Z}^{d}\right):=\left\{a: \mathbb{Z}^{d} \rightarrow \mathbb{C}: \sum_{n \in \mathbb{Z}^{d}}\left|a_{n}\right|^{2}<\infty\right\} .
$$


It is a Hilbert space under the scalar product

$$
(a, b)_{\ell^{2}}:=\sum_{n \in \mathbb{Z}^{d}} a_{n} \bar{b}_{n}
$$

We note that $\left\{E_{p, n}: n \in \mathbb{Z}^{d}\right\}$ defines a complete orthonormal system on $L_{p}^{2}$, i.e.

$$
\left\|\sum_{\substack{n \in \mathbb{Z}^{d} \\\|n\|_{1} \leq m}}\left(f, E_{p, n}\right)_{L_{p}^{2}} E_{p, n}-f\right\|_{L_{p}^{2}} \rightarrow 0, \quad m \rightarrow \infty,
$$

for all $f \in L_{p}^{2}$. The complex numbers

$$
\hat{f}(n):=\left(f, E_{p, n}\right)_{L_{p}^{2}}=\frac{1}{\sharp(p)} \int_{[0, p]} f(x) e^{-2 \pi i\langle n, x \div p\rangle} d x, \quad n \in \mathbb{Z}^{d},
$$

are called the Fourier coefficients of $f$ and they define a mapping from $\mathbb{Z}^{d}$ to $\mathbb{C}$. We will denote this mapping either by $\hat{f}$ or $\mathcal{F}_{d} f$ and call it the $d$-dimensional Fourier transform of $f$. For two functions $\varphi, \psi \in L_{p}^{2}$ we see that

$$
\begin{aligned}
(\varphi, \psi)_{L_{p}^{2}} & =\left(\sum_{n \in \mathbb{Z}^{d}} \hat{\varphi}(n) E_{p, n}, \sum_{m \in \mathbb{Z}^{d}} \hat{\psi}(m) E_{p, m}\right)_{L_{p}^{2}} \\
& =\sum_{n \in \mathbb{Z}^{d}} \sum_{m \in \mathbb{Z}^{d}} \hat{\varphi}(n) \overline{\hat{\psi}(m)}\left(E_{p, n}, E_{p, m}\right)_{L_{p}^{2}} \\
& =\sum_{n \in \mathbb{Z}^{d}} \hat{\varphi}(n) \overline{\hat{\psi}(n)},
\end{aligned}
$$

which is known as Parseval's theorem. From (4.2) we conclude, for the case of $\varphi=\psi$, that

$$
\|\varphi\|_{L_{p}^{2}}^{2}=\sum_{n \in \mathbb{Z}^{d}}\left|\left(\varphi, E_{p, n}\right)_{L_{p}^{2}}\right|^{2}=\|\hat{\varphi}\|_{\ell^{2}}^{2}
$$

This shows that the mapping

$$
\mathcal{F}_{d}: L_{p}^{2} \rightarrow \ell^{2}\left(\mathbb{Z}^{d}\right), \quad \varphi \mapsto\left(\hat{\varphi}_{n}\right)_{n \in \mathbb{Z}^{d}}
$$

is an unitary operator. The inverse operator is given through

$$
\mathcal{F}_{d}^{-1}: \ell^{2}\left(\mathbb{Z}^{d}\right) \rightarrow L_{p}^{2}, \quad\left(a_{n}\right)_{n \in \mathbb{Z}^{d}} \mapsto \sum_{n \in \mathbb{Z}^{d}} a_{n} E_{p, n}
$$

Thus we see the following spectral representation

$$
\varphi=\mathcal{F}_{d}^{-1} \mathcal{F}_{d} \varphi=\sum_{n \in \mathbb{Z}^{d}} \hat{\varphi}_{n} E_{p, n}
$$


for all $\varphi \in L_{p}^{2}$. In analogy to the convolution product of functions on $\mathbb{R}^{d}$, cf. (1.44), we define a convolution product for multi-periodic functions through

$$
(f * g)(x):=\int_{[0, p]} f(x-y) g(y) d y, \quad f, g \in L_{p}^{2} .
$$

One easily verifies that this convolution product is also bilinear, associative and commutative. Furthermore it has the same nice behaviour under Fourier transformation as the convolution product for non-periodic functions, which is one key ingredient for fast matrix-vector multiplications of circulant and Toeplitz matrices.

The analogon of the Convolution theorem for $L^{2}$ (THEOREM 1.43) is stated in the following theorem.

ThEOREM 4.3 (Convolution theorem for periodic functions). For $g, f \in L_{p}^{2}$ it holds that

$$
\mathcal{F}_{d}(f * g)=\sharp(p) \mathcal{F}_{d}(f) \cdot \mathcal{F}_{d}(g) .
$$

Proof. For two functions $f, g \in L_{p}^{2}$ one computes

$$
\begin{aligned}
(f * g)(x) & =\int_{[0, p]} f(x-y) g(y) d y \\
& =\int_{[0, p]}\left(\sum_{n \in \mathbb{Z}^{d}} \hat{f}(n) e^{2 \pi i\langle n, x-y \div p\rangle}\right)\left(\sum_{m \in \mathbb{Z}^{d}} \hat{g}(m) e^{2 \pi i\langle m, y \div p\rangle}\right) d y \\
& =\sum_{n \in \mathbb{Z}^{d}} \sum_{m \in \mathbb{Z}^{d}} \hat{f}(n) \hat{g}(m) \int_{[0, p]} e^{-2 \pi i\langle n, y \div p\rangle} e^{2 \pi i\langle m, y \div p\rangle} d y e^{2 \pi i\langle m, x \div p\rangle} \\
& =\sharp(p) \sum_{n \in \mathbb{Z}^{d}} \hat{f}(n) \hat{g}(n) e^{2 \pi i\langle n, x \div p\rangle} \\
& =\sharp(p) \mathcal{F}_{d}^{-1}\left(\mathcal{F}_{d}(f) \cdot \mathcal{F}_{d}(g)\right)(x),
\end{aligned}
$$

where the dot on the right hand side is the componentwise multiplication in $\ell^{2}$. The interchange of summation and integration is valid as a direct consequence of the continuity of the $L^{2}$ inner product and the convergence of the series representation in $L^{2}$-sense.

\subsubsection{Discrete Fourier transform}

For practical computations with multi-periodic functions, one normally uses trigonometric polynomials of some finite degree. As in most cases it is not possible to find an analytic expression for the Fourier coefficients one cannot simply use a truncated series expansion. Instead one will use trigonometric interpolation polynomials. The results in this section can easily be derived from the case of one-dimensional trigonometric interpolation as it is presented e.g. in [34, Theorem 8.24/8.25]. 
In the case of an equidistantly spaced grid of interpolation points, the coefficients of the interpolation polynomial are given by approximating the integrals (4.1) by the composite trapezoidal rule.

REMARK 4.4. We restrict our presentation to the case of an even number of interpolation points for each space dimension.

We explain this in some more detail for a continuous multi-periodic function $g$ with period-vector $p \in \mathbb{R}_{>0}^{d}$. For $N \in \mathbb{N}^{d}$ we define a regularly spaced grid on $\mathbb{R}^{d}$ through

$$
\left\{x_{j}:=h \odot j: j \in \mathbb{Z}^{d}\right\},
$$

where $h:=p \div 2 N$ denotes the step size. The set of interpolation points in $[0, p]$ that is used for the interpolation is given through

$$
\left\{x_{j}: j=0, \ldots, 2 N-1\right\} .
$$

The uniquely determined trigonometric interpolation polynomial $g_{N}$ of degree $|N|$ that satisfies the interpolation property

$$
g_{N}\left(x_{j}\right)=g\left(x_{j}\right), \quad j=0, \ldots, 2 N-1,
$$

is given through

$$
g_{N}(x):=\sum_{m_{1}=-N_{1}}^{N_{1}-1} \ldots \sum_{m_{d}=-N_{d}}^{N_{d}-1} \tilde{g}\left(m_{1}, \ldots, m_{d}\right) e^{2 \pi i m_{1} x_{1} / p_{1}} \cdot \ldots \cdot e^{2 \pi i m_{d} x_{d} / p_{d}}, \quad x \in \mathbb{R}^{d},
$$

where the discrete Fourier coefficients are given through

$$
\tilde{g}(m):=\frac{1}{\left(2 N_{1}\right) \ldots\left(2 N_{d}\right)} \sum_{j_{1}=0}^{2 N_{1}-1} \ldots \sum_{j_{d}=0}^{2 N_{d}-1} g\left(x_{j_{1}}, \ldots, x_{j_{d}}\right) e^{-2 \pi i m_{d} j_{d} / 2 N_{d}} \cdot \ldots \cdot e^{-2 \pi i m_{1} j_{1} / 2 N_{1}}
$$

for $m_{l}=-N_{l}, \ldots, N_{l}-1$ for $l=1, \ldots, d$. We can write these expressions in a condensed form as

$$
g_{N}(x)=\sum_{m=-N}^{N-1} \tilde{g}(m) e^{2 \pi i\langle m, x \div p\rangle}
$$

and

$$
\tilde{g}(m):=\frac{1}{\sharp(2 N)} \sum_{j=0}^{2 N-1} g\left(x_{j}\right) e^{-2 \pi i\langle m, j \div 2 N\rangle}, \quad m=-N, \ldots, N-1 .
$$

As we mentioned before, the discrete Fourier coefficients are an approximation to the Fourier coefficients given by (4.1). By replacing the integral by the composite 
trapezoidal rule we see that

$$
\begin{aligned}
\hat{g}(m) & =\frac{1}{\sharp(p)} \int_{[0, p]} g(x) e^{-2 \pi i\langle m, x \div p\rangle} d x \\
& \approx \frac{1}{\sharp(p)} \sharp(h) \sum_{j=-N}^{N-1} g\left(x_{j}\right) e^{-2 \pi i\left\langle m, x_{j} \div p\right\rangle} \\
& =\frac{1}{\sharp(p)} \sharp(p \div 2 N) \sum_{j=-N}^{N-1} g\left(x_{j}\right) e^{-2 \pi i\langle m, j \odot h \div p\rangle} \\
& =\frac{1}{\sharp(2 N)} \sum_{j=-N}^{N-1} g\left(x_{j}\right) e^{-2 \pi i\langle m, j \div 2 N\rangle} \\
& =\tilde{g}(m), \quad m=0, \ldots, 2 N-1 .
\end{aligned}
$$

To handle these specific kinds of sums of exponentials, one introduces the following two linear mappings on $\mathbb{C}^{\times(N)}$.

Definition 4.5 (Multi-dimensional DFT/IDFT). For $N \in \mathbb{N}^{d}$ we call the linear mapping $\boldsymbol{F}_{N}: \mathbb{C}^{\times(N)} \rightarrow \mathbb{C}^{\times(N)}$ that maps the d-dimensional array of complex numbers $a:=\left(a_{n}\right)_{n=0, \ldots, N-1}$ onto $\tilde{a}:=\left(\tilde{a}_{n}\right)_{n=0, \ldots, N-1}$, where

$$
\tilde{a}_{m}=\sum_{j=0}^{N-1} a_{j} e^{-2 \pi i\langle m, j \div N\rangle}, \quad m=0, \ldots, N-1,
$$

the $d$-dimensional discrete Fourier transformation $\left(\mathrm{DFT}_{d}\right)$ of length $\sharp(N)$. The linear mapping $\boldsymbol{F}_{N}^{-1}: \mathbb{C}^{\times(N)} \rightarrow \mathbb{C}^{\times(N)}$ that maps the d-dimensional array of complex numbers $\tilde{b}:=\left(\tilde{b}_{n}\right)_{n=0, \ldots, N-1}$ onto $b:=\left(b_{n}\right)_{n=0, \ldots, N-1}$, where

$$
b_{m}=\frac{1}{\sharp(N)} \sum_{j=0}^{N-1} \tilde{b}_{j} e^{2 \pi i\langle m, j \div N\rangle}, \quad m=0, \ldots, N-1,
$$

is called the $d$-dimensional inverse discrete Fourier transformation $\left(\mathrm{IDFT}_{d}\right)$ of length $\sharp(N)$.

We see that the discrete Fourier coefficients $\tilde{g}$ are given as a scaled $\mathrm{DFT}_{d}$ of length $\sharp(2 N)$. The importance of this observation is that there are very efficient algorithms to compute the DFT in $O(\sharp(N) \log \sharp(N))$ operations. These methods are called fast Fourier transform. The ideas date back to Johann Carl Friedrich Gauß.

At last we note that the multi-dimensional discrete Fourier transform is a nested version of one-dimensional discrete Fourier transforms performed over the corresponding dimension of the multi-dimensional data array. 


\subsection{The composite trapezoidal rule}

Throughout this thesis we approximate the integral

$$
Q^{d}[f]:=\int_{\mathbb{R}^{d}} f(x) d x
$$

by the weighted sum

$$
Q_{h}^{d}[f]:=h^{d} \sum_{j \in \mathbb{Z}^{d}} f(h j)
$$

where $h>0$ denotes the step size, i.e. we use the composite trapezoidal rule.

In this section we prove that the composite trapezoidal rule, which coincides with the midpoint rule or rectangular rule on $\mathbb{R}^{d}$, is convergent of high order for certain classes of weighted continuously differentiable functions. Our presentation follows in large parts [40], [42] and [30] extending their results to the case of arbitrary space dimensions.

For the forthcoming analysis we introduce the following spaces of differentiable functions, all of which are Banach spaces equipped with their respective norms.

For $n, d \in \mathbb{N}$ and $p \in \mathbb{R}$ we define

$$
B C_{p}^{n}\left(\mathbb{R}^{d}\right):=\left\{\psi \in B C^{n}\left(\mathbb{R}^{d}\right):\|\psi\|_{B C_{p}^{n}\left(\mathbb{R}^{d}\right)}<\infty\right\}
$$

with

$$
\|\psi\|_{B C_{p}^{n}\left(\mathbb{R}^{d}\right)}:=\max _{|\alpha| \leq n}\left\|w_{p} \partial^{\alpha} \psi\right\|_{B C\left(\mathbb{R}^{d}\right)},
$$

where

$$
w_{p}(x):=(1+|x|)^{p}, \quad x \in \mathbb{R}^{d} .
$$

In other words this means that for $f \in B C_{p}^{n}\left(\mathbb{R}^{d}\right)$, there exists a constant $c>0$ so that

$$
\left|\partial^{\alpha} f(x)\right| \leq \frac{c}{(1+|x|)^{p}}, \quad x \in \mathbb{R}^{d}
$$

and $\alpha \in \mathbb{N}^{d}$ with $|\alpha| \leq n$.

REMARK 4.6. We note the trivial identities

$$
w_{p} w_{q}=w_{p+q}, \quad p, q \in \mathbb{R}
$$

and

$$
w_{-p}=\frac{1}{w_{p}}, \quad p \in \mathbb{R}
$$

Lemma 4.7. Let $f \in B C_{p}^{n}\left(\mathbb{R}^{d}\right)$ with $p>d$. Then $\partial^{\alpha} f \in L^{1}\left(\mathbb{R}^{d}\right)$ for $\alpha \in \mathbb{N}^{d}$ with $|\alpha| \leq n$. 
Proof.

$$
\begin{aligned}
\int_{\mathbb{R}^{d}}\left|\partial^{\alpha} f(x)\right| d x & \leq c \int_{\mathbb{R}^{d}} \frac{1}{(1+|x|)^{p}} d x \\
& =c \omega_{d} \int_{0}^{\infty} \frac{r^{d-1}}{(1+r)^{p}} d r \\
& \leq c \omega_{d} \int_{0}^{\infty}(1+r)^{d-p-1} d r \\
& =\left.c \omega_{d} \frac{1}{d-p}(1+r)^{d-p}\right|_{0} ^{\infty}=\frac{c \omega_{d}}{p-d}<\infty
\end{aligned}
$$

where $\omega_{d}$ denotes the surface area of the unit sphere in $\mathbb{R}^{d}$.

To handle kernel functions on $\mathbb{R}^{d} \times \mathbb{R}^{d}$ we define in an analogue way the space

$$
B C_{p}^{n}\left(\mathbb{R}^{d} \times \mathbb{R}^{d}\right):=\left\{\psi \in B C^{n}\left(\mathbb{R}^{d} \times \mathbb{R}^{d}\right):\|\psi\|_{B C_{p}^{n}\left(\mathbb{R}^{d} \times \mathbb{R}^{d}\right)}<\infty\right\},
$$

with

$$
\|\psi\|_{B C_{p}^{n}\left(\mathbb{R}^{d} \times \mathbb{R}^{d}\right)}:=\max _{|\alpha| \leq n}\left\|\tilde{w}_{p} \partial^{\alpha} \psi\right\|_{B C\left(\mathbb{R}^{d} \times \mathbb{R}^{d}\right)}<\infty
$$

where

$$
\tilde{w}_{p}(x, y):=w_{p}(x-y), \quad x, y \in \mathbb{R}^{d} .
$$

i.e.

$$
\tilde{w}_{p}(x, y)=(1+|x-y|)^{p}, \quad x, y \in \mathbb{R}^{d} .
$$

The following multi-dimensional variant of the Leibniz rule or generalised product rule will be used in the sequel.

Lemma 4.8 (Leibniz rule). For $f, g \in C^{n}\left(\mathbb{R}^{d}\right)$ it holds that

$$
\partial^{\alpha}(f g)=\sum_{0 \leq \beta \leq \alpha}\left(\begin{array}{l}
\alpha \\
\beta
\end{array}\right)\left(\partial^{\alpha-\beta} f\right)\left(\partial^{\beta} g\right)
$$

for all $\alpha \in \mathbb{N}^{d}$ with $|\alpha| \leq n$, where the binomial coefficients for multi-indices $\alpha, \beta \in$ $\mathbb{N}^{d}$ are given through

$$
\left(\begin{array}{l}
\alpha \\
\beta
\end{array}\right):=\prod_{j=1}^{d}\left(\begin{array}{l}
\alpha_{j} \\
\beta_{j}
\end{array}\right) .
$$

Proof. See e.g. [28, p. 247, Theorem 1].

Lemma 4.9. For $\alpha, \beta \in \mathbb{N}^{d}$ with $\beta \leq \alpha$ we have the identity

$$
\sum_{0 \leq \beta \leq \alpha}\left(\begin{array}{c}
\alpha \\
\beta
\end{array}\right)=2^{|\alpha|}, \quad \alpha \in \mathbb{N}^{d} .
$$


Proof. We first note the one-dimensional version of this rule

$$
2^{n}=(1+1)^{n}=\sum_{j=0}^{n}\left(\begin{array}{l}
n \\
j
\end{array}\right) 1^{n-j} 1^{j},
$$

which is a simple consequence of the binomial theorem. The general rule follows by successive summation and the use of (4.15)

$$
\begin{aligned}
\sum_{0 \leq \beta \leq \alpha}\left(\begin{array}{l}
\alpha \\
\beta
\end{array}\right) & =\sum_{\beta_{1}=0}^{\alpha_{1}}\left(\ldots\left(\sum_{\beta_{d-1}=0}^{\alpha_{d-1}}\left(\sum_{\beta_{d}=0}^{\alpha_{d}} \prod_{j=1}^{d}\left(\begin{array}{c}
\alpha_{j} \\
\beta_{j}
\end{array}\right)\right)\right) \ldots\right) \\
& =2^{\alpha_{d}} \sum_{\beta_{1}=0}^{\alpha_{1}}\left(\ldots\left(\sum_{\beta_{d-1}=0}^{\alpha_{d-1}} \prod_{j=1}^{d-1}\left(\begin{array}{c}
\alpha_{j} \\
\beta_{j}
\end{array}\right)\right) \ldots\right)=\ldots= \\
& =2^{\alpha_{d}} \cdot \ldots \cdot 2^{\alpha_{2}} \sum_{\beta_{1}=0}^{\alpha_{1}}\left(\begin{array}{c}
\alpha_{1} \\
\beta_{1}
\end{array}\right)=\prod_{j=1}^{d} 2^{\alpha_{j}}=2^{\alpha_{1}+\ldots \alpha_{d}}=2^{|\alpha|} .
\end{aligned}
$$

The first building block of the error estimate for the composite trapezoidal rule is the next Lemma that examines relations of weighted and non weighted spaces of differentiable functions.

LEMma 4.10. Let $d \in \mathbb{N}, n \in \mathbb{N} \cup\{0\}$.

(i) Let $f \in B C_{p}^{n}\left(\mathbb{R}^{d}\right)$ and $g \in B C_{q}^{n}\left(\mathbb{R}^{d}\right)$ for $p, q \in \mathbb{R}$. Then $f g \in B C_{p+q}^{n}\left(\mathbb{R}^{d}\right)$ for all $x \in \mathbb{R}^{d}$ and

$$
\|f g\|_{B C_{p+q}^{n}\left(\mathbb{R}^{d}\right)} \leq 2^{n}\|f\|_{B C_{p}^{n}\left(\mathbb{R}^{d}\right)}\|g\|_{B C_{q}^{n}\left(\mathbb{R}^{d}\right)} .
$$

(ii) Let $a \in B C_{p}^{n}\left(\mathbb{R}^{d} \times \mathbb{R}^{d}\right)$ and $f \in B C_{q}^{n}\left(\mathbb{R}^{d}\right)$ for $p, q \in \mathbb{R}$. Then $a(x, \cdot) f \in$ $B C_{p+q}^{n}\left(\mathbb{R}^{d}\right)$ for all $x \in \mathbb{R}^{d}$ and

$$
\|a(x, \cdot) f\|_{B C_{p+q}^{n}\left(\mathbb{R}^{d}\right)} \leq 2^{n}\|a(x, \cdot)\|_{B C_{p}^{n}\left(\mathbb{R}^{d}\right)}\|f\|_{B C_{q}^{n}\left(\mathbb{R}^{d}\right)} .
$$

Proof. (i) Using the Leibniz rule and the identity (4.14) we calculate

$$
\begin{aligned}
\left|\partial^{\alpha}(f g)(x)\right| & =\left|\sum_{0 \leq \beta \leq \alpha}\left(\begin{array}{l}
\alpha \\
\beta
\end{array}\right)\left(\partial^{\alpha-\beta} f\right)(x)\left(\partial^{\beta} g\right)(x)\right| \\
& =\frac{1}{w_{p}(x) w_{q}(x)}\left|\sum_{0 \leq \beta \leq \alpha}\left(\begin{array}{l}
\alpha \\
\beta
\end{array}\right)\left[w_{p}(x)\left(\partial^{\alpha-\beta} f\right)(x)\right]\left[w_{q}(x)\left(\partial^{\beta} g\right)(x)\right]\right| \\
& \leq \frac{1}{w_{p}(x) w_{q}(x)} \sum_{0 \leq \beta \leq \alpha}\left(\begin{array}{l}
\alpha \\
\beta
\end{array}\right)\|f\|_{B C_{p}^{n}\left(\mathbb{R}^{d}\right)}\|g\|_{B C_{q}^{n}\left(\mathbb{R}^{d}\right)} \\
& =\frac{2^{|\alpha|}}{w_{p}(x) w_{q}(x)}\|f\|_{B C_{p}^{n}\left(\mathbb{R}^{d}\right)}\|g\|_{B C_{q}^{n}\left(\mathbb{R}^{d}\right)}
\end{aligned}
$$


for all $\alpha \in \mathbb{N}^{d}$ with $|\alpha| \leq n$. We use that $w_{p} w_{q}=w_{p+q}$ for $p, q \in \mathbb{R}$, cf. REMARK 4.6, multiply this inequality with $w_{p+q}(x)$ and take the supremum over $x \in \mathbb{R}^{d}$. This yields the estimate (4.16).

(ii) This is a direct consequence of (i), if we first note that for $a \in B C_{p}^{n}\left(\mathbb{R}^{d} \times \mathbb{R}^{d}\right)$ it holds that $a(x, \cdot) \in B C_{p}^{n}\left(\mathbb{R}^{d}\right)$ for all $x \in \mathbb{R}^{d}$.

The second building block it the following Lemma, which is a consequence of a generalisation of the Euler-Maclaurin expansion for the multi-dimensional case. Let

$$
C_{0}^{n}\left([0,1]^{d}\right):=\left\{\psi \in C^{n}\left([0,1]^{d}\right):\left.\partial^{\alpha} \psi\right|_{\partial\left([0,1]^{d}\right)}=0 \text { for }|\alpha| \leq n\right\},
$$

the space of $n$-times continuously differentiable functions on $[0,1]^{d}$ that vanish with all their derivatives up to order $n$ on the boundary.

Lemma 4.11. Let $m, n \in \mathbb{N}, g \in C_{0}^{m}\left([0,1]^{d}\right)$ and define $h:=1 / n$ and $N:=$ $(n, \ldots, n) \in \mathbb{N}^{d}$. Then

$$
\left|\int_{[0,1]^{d}} g(x) d x-h^{d} \sum_{j=1}^{N-1} g(h j)\right| \leq C\|g\|_{C^{m}\left([0,1]^{d}\right)} h^{m},
$$

where the constant $C>0$ depends only on $m$.

Proof. For details of a proof see e.g. the comments in [51].

The following theorem is an extension of [42, Lemma 3.10] to the multi-dimensional case and the proof presented given here closely follows the one in [42].

LEMmA 4.12. If, for some $p>d$ and $m \in \mathbb{N}, f \in B C_{p}^{m}\left(\mathbb{R}^{d}\right)$, then

$$
\left|Q^{d} f-Q_{h}^{d} f\right| \leq C\|f\|_{B C_{p}^{m}\left(\mathbb{R}^{d}\right)} h^{m}, \quad h>0,
$$

where the constant $C>0$ depends only on $m$ and $p$.

Proof. Let $\phi \in C^{\infty}(\mathbb{R})$ be such that $\phi(s)=-\frac{1}{2}$ for $s \leq 0$ and $\phi(s)=\frac{1}{2}$ for $s \geq 1$. Let $\psi_{0}(s):=\phi(s)-\phi(s-1)$ and let $\psi_{j}(s):=\psi_{0}(s-j)$ for $j \in \mathbb{Z}$. Then $\psi_{j} \in C_{\text {comp }}^{\infty}(\mathbb{R})$, with $\operatorname{supp} \psi_{j}=[j, 2+j]$ and

$$
\sum_{j \in \mathbb{Z}} \psi_{j}(s)=1, \quad s \in \mathbb{R}
$$

so that we have a partition of unity on $\mathbb{R}$. The family of functions

$$
\Psi_{j}(x):=\psi_{j_{1}}\left(x_{1}\right) \cdot \ldots \cdot \psi_{j_{d}}\left(x_{d}\right), \quad j \in \mathbb{Z}^{d}, \quad x \in \mathbb{R}^{d} .
$$


defines a smooth partition of unity on $\mathbb{R}^{d}$ and $\operatorname{supp} \Psi_{j}=[j, 2+j] \subset \mathbb{R}^{d}$. Let, for $h>0$ and $j \in \mathbb{Z}^{d}, e_{j}(h):=Q^{d}\left[\Psi_{j} f\right]-Q_{h}^{d}\left[\Psi_{j} f\right]$. Then

$$
Q^{d}[f]-Q_{h}^{d}[f]=\sum_{j \in \mathbb{Z}^{d}} e_{j}(h)
$$

and by Lemma 4.11 and Lemma 4.10,

$$
\begin{aligned}
\left|e_{j}(h)\right| & \leq C\left\|\Psi_{j} f\right\|_{B C^{m}\left(\operatorname{supp} \Psi_{j}\right)} h^{m} \\
& \leq 2^{m} C\left\|\Psi_{j}\right\|_{B C^{m}\left(\operatorname{supp} \Psi_{j}\right)}\|f\|_{B C^{m}\left(\operatorname{supp} \Psi_{j}\right)} h^{m} \\
& \leq 2^{m} C\left\|\Psi_{j}\right\|_{B C^{m}\left(\operatorname{supp} \Psi_{j}\right)} \max _{t \in \operatorname{supp} \Psi_{j}} \frac{1}{w_{p}(t)}\left\|w_{p} f\right\|_{B C^{m}\left(\operatorname{supp} \Psi_{j}\right)} h^{m} \\
& \leq 2^{m} C h^{m}\left\|\Psi_{0}\right\|_{\left(\operatorname{supp} \Psi_{0}\right)}\|f\|_{B C_{p}^{m}\left(\mathbb{R}^{d}\right)} \max _{t \in \operatorname{supp} \Psi_{j}}(1+|t|)^{-p} \\
& \leq C_{m} h^{m}\|f\|_{B C_{p}^{m}\left(\mathbb{R}^{d}\right)} \max _{t \in[j, 2+j]}(1+|t|)^{-p},
\end{aligned}
$$

where $C_{m}$ depends only on $m$ and on $\left\|\Psi_{0}\right\|_{C^{m}([0,2])}$. Thus,

$$
\begin{aligned}
\left|Q^{d}[f]-Q_{h}^{d}[f]\right| & \leq \sum_{j \in \mathbb{Z}^{d}}\left|e_{j}(h)\right| \\
& \leq C_{m} h^{m}\|f\|_{B C_{p}^{m}\left(\mathbb{R}^{d}\right)} \sum_{j \in \mathbb{Z}^{d}} \max _{t \in[j, 2+j]}(1+|t|)^{-p} \\
& \leq C\|f\|_{B C_{p}^{m}\left(\mathbb{R}^{d}\right)} h^{m},
\end{aligned}
$$

where $C>0$ depends only on $m$ and $p$. The convergence of the multi-dimensional series can be concluded from a generalisation of the integral test. We first note that the function $w_{p}: \mathbb{R}_{>0}^{d} \rightarrow \mathbb{R}, t \mapsto(1+|t|)^{-p}$ is an integrable and non-negative monotone decreasing function, which means that the maximum of $w_{p}$ over the interval $[j, j+2]$ is attained at the left end point, i.e.

$$
\max _{t \in[j, 2+j]}(1+|t|)^{-p}=(1+|j|)^{-p} .
$$

Using symmetry properties we can write the series in the form

$$
\sum_{j \in \mathbb{Z}^{d}} \max _{t \in[j, 2+j]}(1+|t|)^{-p}=2^{d} \sum_{j \in \mathbb{Z}_{>0}^{d}}(1+|j|)^{-p}=2^{d} \sum_{j \in \mathbb{Z}_{>0}^{d}} w_{p}(j) .
$$

The convergence of the series now follows from the estimate

$$
w_{p}(j) \leq \int_{[j, j+1]} w_{p}(t) d t, \quad j \in \mathbb{Z}_{>0}^{d},
$$

which is a direct consequence of the monotonicity and which implies that

$$
\sum_{j \in \mathbb{Z}_{>0}^{d}} w_{p}(j) \leq \sum_{j \in \mathbb{Z}_{>0}^{d}} \int_{[j, j+1]} w_{p}(t) d t=\int_{\mathbb{R}_{>0}^{d}} w_{p}(t) d t<\infty .
$$


Chapter 4 Tools 


\section{Chapter 5}

\section{Operator approximations}

In this chapter we analyse operator approximations that can be used either

- in the context of Nyström methods together with the finite section method as a truncation scheme

or

- in the context of the recently proposed multi-section method, where, in contrast to the classical Nyström method, a linear least squares problem has to be solved.

We are interested in approximating integral operators

$$
(A \psi)(\boldsymbol{x}):=\int_{\mathbb{R}^{2}} K(\boldsymbol{x}, \boldsymbol{y}) J(\boldsymbol{y}) \psi(\boldsymbol{y}) d \boldsymbol{y}, \quad \boldsymbol{x} \in \mathbb{R}^{2},
$$

where $J(\boldsymbol{y})=\sqrt{1+|\nabla f(\boldsymbol{y})|^{2}}$ denotes as usual the surface area element, by operators of the form

$$
\left(A_{h} \psi\right)(\boldsymbol{x}):=\sum_{\boldsymbol{j} \in \mathbb{Z}^{2}} K_{h, \boldsymbol{j}}(\boldsymbol{x}) J(h \boldsymbol{j}) \psi(h \boldsymbol{j}), \quad \boldsymbol{x} \in \mathbb{R}^{2}, h>0,
$$

where $K_{h, \boldsymbol{j}}, \boldsymbol{j} \in \mathbb{Z}^{2}$, is a family of regular functions that have to be determined.

For a reasonable large class of kernel functions and densities one can get an approximation of the form (5.2) by replacing the integral by the composite trapezoidal rule. Thus we get

$$
\left(A_{h} \psi\right)(\boldsymbol{x})=Q_{h}^{2}[K(\boldsymbol{x}, \cdot) J \psi]=\sum_{\boldsymbol{j} \in \mathbb{Z}^{2}} K_{h, \boldsymbol{j}}(\boldsymbol{x}) J(h \boldsymbol{j}) \psi(h \boldsymbol{j}), \quad \boldsymbol{x} \in \mathbb{R}^{2}
$$

where

$$
K_{h, \boldsymbol{j}}(\boldsymbol{x}):=h^{2} K(\boldsymbol{x}, h \boldsymbol{j}), \quad \boldsymbol{j} \in \mathbb{Z}^{2} .
$$

These kind of operator approximations are used in Nyström methods and it is known that the operator $A_{h}$ cannot converge in norm to the original operator $A$ for $h \rightarrow 0$. The best one can hope for instead is pointwise convergence, i.e.

$$
\left\|A \psi-A_{h} \psi\right\| \rightarrow 0, \quad h \rightarrow 0,
$$

for a reasonable class of functions $\psi$ and an appropriate norm $\|\cdot\|$. 


\subsection{Approximations for weighted differentiable kernels}

In the case that the kernel and density exhibits a certain rate of decay, we are able to prove convergence and quantify a convergence rate.

LEMMA 5.1. Let $A$ be given through (5.1) with $K \in B C_{p}^{m}\left(\mathbb{R}^{d} \times \mathbb{R}^{d}\right), \psi \in B C_{q}^{m}\left(\mathbb{R}^{d}\right)$ with $p+q \geq d$ and $f \in B C^{m+1}\left(\mathbb{R}^{d}\right)$ for some $m \in \mathbb{N}$. Then, the operator $A_{h}$ for $h>0$ given through (5.2), with $K_{h, j}$ given through (5.3), converges pointwise to $A$ and the error can be estimated through

$$
\left\|A \psi-A_{h} \psi\right\|_{B C\left(\mathbb{R}^{d}\right)} \leq C\|K(\boldsymbol{x}, \cdot)\|_{B C_{p}^{m}\left(\mathbb{R}^{d}\right)}\|J\|_{B C^{m}\left(\mathbb{R}^{d}\right)}\|\psi\|_{B C_{q}^{m}\left(\mathbb{R}^{d}\right)} h^{m},
$$

for some constant $C>0$ dependent only on $m$ and $p$.

Proof. For $f \in B C^{m+1}\left(\mathbb{R}^{d}\right)$ we see that $J \in B C^{m}\left(\mathbb{R}^{d}\right)$ so that LEMMA 4.10 yields $K(\boldsymbol{x}, \cdot) J \psi \in B C_{p+q}^{m}\left(\mathbb{R}^{d}\right)$. Lemma 4.7 ensures that $K(\boldsymbol{x}, \cdot) J \psi \in L^{1}\left(\mathbb{R}^{d}\right)$ for all $\boldsymbol{x} \in \mathbb{R}^{d}$, as $p+q>d$. Hence Lemma 4.12 yields

$$
\begin{aligned}
\left\|A \psi-A_{h} \psi\right\|_{B C\left(\mathbb{R}^{d}\right)} & =\sup _{\boldsymbol{x} \in \mathbb{R}^{d}}\left|Q^{d}[K(\boldsymbol{x}, \cdot) J \psi]-Q_{h}^{d}[K(\boldsymbol{x}, \cdot) J \psi]\right| \\
& \leq c\|K(\boldsymbol{x}, \cdot) J \psi\|_{B C_{p+q}^{m}\left(\mathbb{R}^{d}\right)} h^{m}
\end{aligned}
$$

so that the bound (5.5) follows from LEMMA 4.10.

REMARK 5.2. The reason for our interest in this particular case is that the global part of the single-layer and double-layer operator satisfy these conditions for $d=2$ and $p=2$, as we have shown in the estimates (1.22) and (1.28). To retain the convergence order, it suffices that the density satisfies the mild decay property $\psi \in$ $B C_{q}^{m}\left(\mathbb{R}^{2}\right)$ for $q>0$.

\subsection{Approximations for weakly singular kernels}

In this section we develop a quadrature scheme that is suitable for the weakly singular integral kernels of the single-layer and double-layer potential operators.

Thus we consider the case where the operator $A$, given through (5.1), has the kernel $K=k^{S}$ or $K=k^{K}$, with

$$
\begin{aligned}
k^{S}(\boldsymbol{x}, \boldsymbol{y}) & :=2 G(x, y), \\
k^{K}(\boldsymbol{x}, \boldsymbol{y}) & :=2 \frac{\partial G(x, y)}{\partial \nu(y)},
\end{aligned}
$$

for $x=(\boldsymbol{x}, f(\boldsymbol{x}))$ and $y=(\boldsymbol{y}, f(\boldsymbol{y}))$ and $G$ given through $(10)$. 
We start our explanations, by first deriving suitable decompositions of the two kernel functions in the form

$$
K(\boldsymbol{x}, \boldsymbol{y}):=K_{1}(\boldsymbol{x}, \boldsymbol{y})+\frac{1}{|\boldsymbol{x}-\boldsymbol{y}|} K_{2}(\boldsymbol{x}, \boldsymbol{y}), \quad \boldsymbol{x} \neq \boldsymbol{y},
$$

where we assume that, in the case that $f$ is a real analytical function, the following two conditions hold:

(W1) the kernel function $K_{1}$ is smooth,

(W2) the function $\tilde{K}_{2, \boldsymbol{x}}(r, \theta):=K_{2}(\boldsymbol{x}, \boldsymbol{x}+r p(\theta))$, for $r \in \mathbb{R}, \theta \in[0,2 \pi)$ possesses continuous partial derivatives in angular and radial direction of arbitrary order, i.e. $\tilde{K}_{2, \boldsymbol{x}}$ is a smooth function in the polar domain $\mathbb{R} \times[0,2 \pi)$, where

$$
p(\theta):=(\cos \theta, \sin \theta) .
$$

\subsubsection{The case of the single-layer potential}

To derive a decomposition of the form (5.8), we introduce the kernel functions

$$
k^{S, 1}(\boldsymbol{x}, \boldsymbol{y}):=\frac{1}{2 \pi} \frac{\exp \left[i \kappa\left(|\boldsymbol{x}-\boldsymbol{y}|^{2}+[f(\boldsymbol{x})-f(\boldsymbol{y})]^{2}\right)^{1 / 2}\right]}{\left(|\boldsymbol{x}-\boldsymbol{y}|^{2}+[f(\boldsymbol{x})-f(\boldsymbol{y})]^{2}\right)^{1 / 2}}
$$

and

$$
k^{S, 2}(\boldsymbol{x}, \boldsymbol{y}):=\frac{1}{2 \pi} \frac{\exp \left[i \kappa\left(|\boldsymbol{x}-\boldsymbol{y}|^{2}+[f(\boldsymbol{x})+f(\boldsymbol{y})]^{2}\right)^{1 / 2}\right]}{\left(|\boldsymbol{x}-\boldsymbol{y}|^{2}+[f(\boldsymbol{x})+f(\boldsymbol{y})]^{2}\right)^{1 / 2}} .
$$

To handle these kernel functions more easily we denote the distance $|x-y|$ respectively $\left|x-y^{\prime}\right|$ of two points $x, y \in \Gamma_{f}$ by

$$
d(\boldsymbol{x}, \boldsymbol{y}):=\left(|\boldsymbol{x}-\boldsymbol{y}|^{2}+[f(\boldsymbol{x})-f(\boldsymbol{y})]^{2}\right)^{1 / 2},
$$

and

$$
d^{\prime}(\boldsymbol{x}, \boldsymbol{y}):=\left(|\boldsymbol{x}-\boldsymbol{y}|^{2}+[f(\boldsymbol{x})+f(\boldsymbol{y})]^{2}\right)^{1 / 2}
$$

The function

$$
c(\boldsymbol{x}, \boldsymbol{y}):=\left(1+\left[\frac{f(\boldsymbol{x})-f(\boldsymbol{y})}{|\boldsymbol{x}-\boldsymbol{y}|}\right]^{2}\right)^{1 / 2}, \quad \boldsymbol{x} \neq \boldsymbol{y},
$$

can be written as

$$
d(\boldsymbol{x}, \boldsymbol{y})=|\boldsymbol{x}-\boldsymbol{y}| c(\boldsymbol{x}, \boldsymbol{y}), \quad \boldsymbol{x} \neq \boldsymbol{y} .
$$


This yields the decomposition

$$
k^{S}(\boldsymbol{y}, \boldsymbol{y})=k^{S, 1}(\boldsymbol{x}, \boldsymbol{y})-k^{S, 2}(\boldsymbol{x}, \boldsymbol{y})=k_{1}^{S}(\boldsymbol{x}, \boldsymbol{y})+\frac{1}{|\boldsymbol{x}-\boldsymbol{y}|} k_{2}^{S}(\boldsymbol{x}, \boldsymbol{y})
$$

with

$$
k_{1}^{S}(\boldsymbol{x}, \boldsymbol{y}):=\left(-k^{S, 2}(\boldsymbol{x}, \boldsymbol{y})+i \frac{\sin [\kappa d(\boldsymbol{x}, \boldsymbol{y})]}{2 \pi d(\boldsymbol{x}, \boldsymbol{y})}\right)
$$

and

$$
k_{2}^{S}(\boldsymbol{x}, \boldsymbol{y}):=\frac{\cos [\kappa d(\boldsymbol{x}, \boldsymbol{y})]}{2 \pi c(\boldsymbol{x}, \boldsymbol{y})}
$$

The regular part. In the case that $f$ is an analytic function, we conclude that

- $J=\sqrt{1+|\nabla f|^{2}} \in B C^{\infty}\left(\mathbb{R}^{2}\right)$,

- $c(\boldsymbol{x}, \cdot), d(\boldsymbol{x}, \cdot) \in B C^{\infty}\left(\mathbb{R}^{2} \backslash\{\boldsymbol{x}\}\right)$ for all $\boldsymbol{x} \in \mathbb{R}^{2}$,

- $d^{\prime}(\boldsymbol{x}, \cdot)$ is an analytic function for all $\boldsymbol{x} \in \mathbb{R}^{2}$.

Furthermore we have the bounds

$$
2 f^{-} \leq d^{\prime}(\boldsymbol{x}, \boldsymbol{y}) \quad \text { and } \quad 1 \leq c(\boldsymbol{x}, \boldsymbol{y}) \leq\left(1+L_{f}^{2}\right)^{1 / 2}
$$

for $\boldsymbol{x}, \boldsymbol{y} \in \mathbb{R}^{2}$.

The function $z \mapsto e^{i \kappa z} / z, z \in \mathbb{C}$ is meromorph in $\mathbb{C} \backslash\{0\}$. Thus we conclude, together with the bound on $d^{\prime}$, that $k^{S, 2}$ is analytic as composition of analytic functions.

The function

$$
y \mapsto \sin (|y|) /|y|, \quad y \in \mathbb{R}^{3}
$$

is a real analytic function, hence also the function

$$
\boldsymbol{y} \mapsto \sin [d(\boldsymbol{x}, \boldsymbol{y})] / d(\boldsymbol{x}, \boldsymbol{y}), \quad \boldsymbol{x} \in \mathbb{R}^{2}
$$

Thus $k_{1}^{S}(\boldsymbol{x}, \cdot) \in B C^{\infty}\left(\mathbb{R}^{2}\right)$ for all $\boldsymbol{x} \in \mathbb{R}^{2}$.

The discontinuous part. It remains to show that the function

$$
\tilde{k}_{2, \boldsymbol{x}}^{S}(r, \theta):=k_{2}^{S}(\boldsymbol{x}, \boldsymbol{x}+r p(\theta)), \quad r \in \mathbb{R}, \theta \in[0,2 \pi)
$$


possesses continuous partial derivatives of arbitrary order. For this it suffices to consider the function $c$, given through (5.14). Introducing polar coordinates with centre $\boldsymbol{x}$ we can write $c$ as a function in the polar domain, i.e.

$$
\tilde{c}_{\boldsymbol{x}}(r, \theta):=\left(1+\left[\frac{f(\boldsymbol{x})-f(\boldsymbol{x}+r p(\theta))}{r}\right]^{2}\right)^{1 / 2}, \quad r>0, \theta \in[0,2 \pi) .
$$

With the help of the Taylor expansion

$$
f(\boldsymbol{x}+r p(\theta))=f(\boldsymbol{x})+\langle\nabla f(\boldsymbol{x}), p(\theta)\rangle r+\sum_{|\alpha| \geq 2} \frac{1}{\alpha !}\left(\partial^{\alpha} f\right)(\boldsymbol{x}) p(\theta)^{\alpha} r^{\alpha}
$$

we see that the function

$$
(r, \theta) \mapsto \frac{f(\boldsymbol{x})-f(\boldsymbol{x}+r p(\theta))}{r}=-\langle\nabla f(\boldsymbol{x}), p(\theta)\rangle-\sum_{|\alpha| \geq 2}\left(\partial^{\alpha} f\right)(\boldsymbol{x}) p(\theta)^{\alpha} r^{\alpha-1}
$$

possesses continuous partial derivatives of arbitrary order. Furthermore we see that

$$
\begin{aligned}
\lim _{r \rightarrow 0} \tilde{k}_{2, \boldsymbol{x}}^{S}(r, \theta) & =\lim _{r \rightarrow 0} \frac{1}{2 \pi} \frac{\cos \left[\kappa r \tilde{c}_{\boldsymbol{x}}(r, \theta)\right]}{\tilde{c}_{\boldsymbol{x}}(r, \theta)}=\lim _{r \rightarrow 0} \frac{1}{2 \pi} \frac{1}{\tilde{c}_{\boldsymbol{x}}(r, \theta)} \\
& =\frac{1}{2 \pi}\left(1+[\langle\nabla f(\boldsymbol{x}), p(\theta)\rangle]^{2}\right)^{-1 / 2}
\end{aligned}
$$

\subsubsection{The case of the double-layer potential}

To derive the decomposition for the double-layer potential, we introduce the kernel functions

$$
\begin{aligned}
k^{K, 1}(\boldsymbol{x}, \boldsymbol{y}):=\frac{1}{2 \pi} \frac{\langle(-\nabla f(\boldsymbol{y}), 1),(\boldsymbol{x}-\boldsymbol{y}, f(\boldsymbol{x})-f(\boldsymbol{y}))\rangle[J(\boldsymbol{y})]^{-1}}{\left(|\boldsymbol{x}-\boldsymbol{y}|^{2}+[f(\boldsymbol{x})-f(\boldsymbol{y})]^{2}\right)} \\
\cdot\left(1-i \kappa\left(|\boldsymbol{x}-\boldsymbol{y}|^{2}+[f(\boldsymbol{x})-f(\boldsymbol{y})]^{2}\right)^{1 / 2}\right) \\
\cdot \frac{\exp \left[i \kappa\left(|\boldsymbol{x}-\boldsymbol{y}|^{2}+[f(\boldsymbol{x})-f(\boldsymbol{y})]^{2}\right)^{1 / 2}\right]}{\left(|\boldsymbol{x}-\boldsymbol{y}|^{2}+[f(\boldsymbol{x})-f(\boldsymbol{y})]^{2}\right)^{1 / 2}}
\end{aligned}
$$

and

$$
\begin{aligned}
k^{K, 2}(\boldsymbol{x}, \boldsymbol{y}):= & \frac{1}{2 \pi} \frac{\langle(-\nabla f(\boldsymbol{y}),-1),(\boldsymbol{x}-\boldsymbol{y}, f(\boldsymbol{x})+f(\boldsymbol{y}))\rangle[J(\boldsymbol{y})]^{-1}}{\left(|\boldsymbol{x}-\boldsymbol{y}|^{2}+[f(\boldsymbol{x})+f(\boldsymbol{y})]^{2}\right)} \\
& \cdot\left(1-i \kappa\left(|\boldsymbol{x}-\boldsymbol{y}|^{2}+[f(\boldsymbol{x})+f(\boldsymbol{y})]^{2}\right)^{1 / 2}\right) \\
& \cdot \frac{\exp \left[i \kappa\left(|\boldsymbol{x}-\boldsymbol{y}|^{2}+[f(\boldsymbol{x})+f(\boldsymbol{y})]^{2}\right)^{1 / 2}\right]}{\left(|\boldsymbol{x}-\boldsymbol{y}|^{2}+[f(\boldsymbol{x})+f(\boldsymbol{y})]^{2}\right)^{1 / 2}}
\end{aligned}
$$


Note that

$$
k^{K, 1}(\boldsymbol{x}, \boldsymbol{y})=\frac{1}{2 \pi} \frac{\langle\nu(y), x-y\rangle}{|x-y|^{2}}(1-i \kappa|x-y|) \frac{\exp [i \kappa|x-y|]}{|x-y|}=2 \frac{\partial \Phi(x, y)}{\partial \nu(y)}
$$

and

$$
k^{K, 2}(\boldsymbol{x}, \boldsymbol{y})=\frac{1}{2 \pi} \frac{\left\langle[\nu(y)]^{\prime}, x-y^{\prime}\right\rangle}{\left|x-y^{\prime}\right|^{2}}\left(1-i \kappa\left|x-y^{\prime}\right|\right) \frac{\exp \left[i \kappa\left|x-y^{\prime}\right|\right]}{\left|x-y^{\prime}\right|}=2 \frac{\partial \Phi\left(x, y^{\prime}\right)}{\partial \nu(y)}
$$

To write these functions more compactly we introduce the functions

$$
a(\boldsymbol{x}, \boldsymbol{y}):=\frac{(f(\boldsymbol{x})-f(\boldsymbol{y})-\langle\nabla f(\boldsymbol{y}), \boldsymbol{x}-\boldsymbol{y}\rangle)[J(\boldsymbol{y})]^{-1}}{d(\boldsymbol{x}, \boldsymbol{y})^{2}}, \quad \boldsymbol{x} \neq \boldsymbol{y},
$$

and

$$
a^{\prime}(\boldsymbol{x}, \boldsymbol{y}):=\frac{-(f(\boldsymbol{x})+f(\boldsymbol{y})+\langle\nabla f(\boldsymbol{y}), \boldsymbol{x}-\boldsymbol{y}\rangle)[J(\boldsymbol{y})]^{-1}}{d^{\prime}(\boldsymbol{x}, \boldsymbol{y})^{2}} .
$$

Thus

$$
k^{K, 1}(\boldsymbol{x}, \boldsymbol{y})=\frac{a(\boldsymbol{x}, \boldsymbol{y})}{2 \pi}(1-i \kappa d(\boldsymbol{x}, \boldsymbol{y})) \frac{\exp [i \kappa d(\boldsymbol{x}, \boldsymbol{y})]}{d(\boldsymbol{x}, \boldsymbol{y})}
$$

and

$$
k^{K, 2}(\boldsymbol{x}, \boldsymbol{y})=\frac{a^{\prime}(\boldsymbol{x}, \boldsymbol{y})}{2 \pi}\left(1-i \kappa d^{\prime}(\boldsymbol{x}, \boldsymbol{y})\right) \frac{\exp \left[i \kappa d^{\prime}(\boldsymbol{x}, \boldsymbol{y})\right]}{d^{\prime}(\boldsymbol{x}, \boldsymbol{y})} .
$$

Now, using (5.15), we can write the kernel function of the double-layer potential as

$$
k^{K}(\boldsymbol{y}, \boldsymbol{y})=k^{K, 1}(\boldsymbol{x}, \boldsymbol{y})-k^{K, 2}(\boldsymbol{x}, \boldsymbol{y})=k_{1}^{K}(\boldsymbol{x}, \boldsymbol{y})+\frac{1}{|\boldsymbol{x}-\boldsymbol{y}|} k_{2}^{K}(\boldsymbol{x}, \boldsymbol{y}),
$$

where

$$
k_{1}^{K}(\boldsymbol{x}, \boldsymbol{y}):=-k^{K, 2}(\boldsymbol{x}, \boldsymbol{y})
$$

and

$$
k_{2}^{K}(\boldsymbol{x}, \boldsymbol{y}):=\frac{a(\boldsymbol{x}, \boldsymbol{y})}{2 \pi}(1-i \kappa d(\boldsymbol{x}, \boldsymbol{y})) \frac{\exp [i \kappa d(\boldsymbol{x}, \boldsymbol{y})]}{c(\boldsymbol{x}, \boldsymbol{y})} .
$$

An analysis, similar to the one we did before in the case of the single-layer operator, yields $k_{1}^{K} \in B C^{\infty}\left(\mathbb{R}^{2}\right)$ for an analytic function $f$. In addition we see that it suffices to study the function $a$ given through (5.22). The Taylor expansion (5.19) yields

$$
f(\boldsymbol{x})-f(\boldsymbol{y})-\langle\nabla f(\boldsymbol{y}), \boldsymbol{x}-\boldsymbol{y}\rangle=\sum_{|\alpha| \geq 2} \frac{1}{\alpha !}\left(\partial^{\alpha} f\right)(\boldsymbol{x})(\boldsymbol{x}-\boldsymbol{y})^{\alpha},
$$


so that

$$
a(\boldsymbol{x}, \boldsymbol{y})=\frac{\sum_{|\alpha| \geq 2} \frac{1}{\alpha !}\left(\partial^{\alpha} f\right)(\boldsymbol{x})(\boldsymbol{x}-\boldsymbol{y})^{\alpha}}{|\boldsymbol{x}-\boldsymbol{y}|^{2}[c(\boldsymbol{x}, \boldsymbol{y})]^{2}}[J(\boldsymbol{y})]^{-1} .
$$

Thus we see that the function

$$
\tilde{a}_{\boldsymbol{x}}(r, \theta):=a(\boldsymbol{x}, \boldsymbol{x}+r p(\theta))
$$

can be written as

$$
\tilde{a}_{\boldsymbol{x}}(r, \theta)=\sum_{|\alpha| \geq 2} \frac{1}{\alpha !}\left(\partial^{\alpha} f\right)(\boldsymbol{x}) p(\theta)^{\alpha} r^{\alpha-2}\left[\tilde{c}_{\boldsymbol{x}}(r, \theta)\right]^{-2}[J(\boldsymbol{x}+r p(\theta))]^{-1},
$$

which is clearly a differentiable function in the polar domain. This proves that

$$
\tilde{k}_{2, \boldsymbol{x}}^{K}(r, \theta):=k_{2}^{K}(\boldsymbol{x}, \boldsymbol{x}+\operatorname{rp}(\theta))
$$

is a smooth function in the polar domain with

$$
\lim _{r \rightarrow 0} \tilde{k}_{2, \boldsymbol{x}}^{K}(r, \theta)=\frac{1}{2 \pi} \sum_{|\alpha|=2} \frac{1}{\alpha !}\left(\partial^{\alpha} f\right)(\boldsymbol{x}) p(\theta)^{\alpha}\left[\tilde{c}_{\boldsymbol{x}}(0, \theta)\right]^{-3}[J(\boldsymbol{x}+r p(\theta))]^{-1} .
$$

\subsubsection{The locally corrected quadrature scheme}

The decomposition (5.8) is our starting point for a second decomposition that truncates the singular part to a function with compact support. To this end we introduce a $n$-times continuously differentiable, symmetric cut-off function $\chi_{a, b}: \mathbb{R} \rightarrow[0,1]$ for some constants $0<a<b$ such that

$$
0 \leq \chi_{a, b}(t) \leq 1, \quad t \in \mathbb{R}
$$

and

$$
\chi_{a, b}(t)= \begin{cases}1, & |t|<a \\ 0, & |t|>b\end{cases}
$$

A typical example of such a cut-off function is shown in FigurE 5.1. With the help of such a cut-off function we define a second decomposition

$$
K(\boldsymbol{x}, \boldsymbol{y})=K_{\text {local }}(\boldsymbol{x}, \boldsymbol{y})+K_{\text {global }}(\boldsymbol{x}, \boldsymbol{y}),
$$

where

$$
\begin{aligned}
K_{\text {local }}(\boldsymbol{x}, \boldsymbol{y}) & :=\chi_{a, b}(|\boldsymbol{x}-\boldsymbol{y}|) \frac{1}{|\boldsymbol{x}-\boldsymbol{y}|} K_{2}(\boldsymbol{x}, \boldsymbol{y}), \\
K_{\text {global }}(\boldsymbol{x}, \boldsymbol{y}) & :=K_{1}(\boldsymbol{x}, \boldsymbol{y})+\left[1-\chi_{a, b}(|\boldsymbol{x}-\boldsymbol{y}|)\right] \frac{1}{|\boldsymbol{x}-\boldsymbol{y}|} K_{2}(\boldsymbol{x}, \boldsymbol{y}) .
\end{aligned}
$$




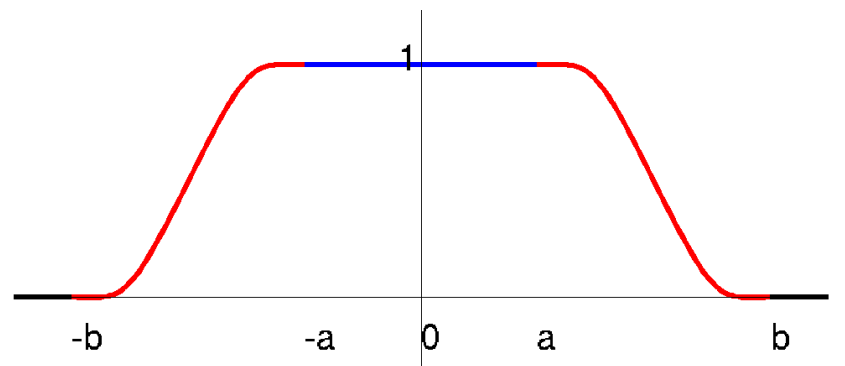

Figure 5.1: An example for a cut-off function $\chi_{a, b}$

Hence we can write (5.1) as the sum of $A_{\mathrm{g}}$ and $A_{\mathrm{l}}$, where

$$
\begin{aligned}
\left(A_{\mathrm{g}} \psi\right)(\boldsymbol{x}) & :=\int_{\mathbb{R}^{2}} K_{\text {global }}(\boldsymbol{x}, \boldsymbol{y}) J(\boldsymbol{y}) \psi(\boldsymbol{y}) d \boldsymbol{y}, \quad \boldsymbol{x} \in \mathbb{R}^{2}, \\
\left(A_{\mathrm{l}} \psi\right)(\boldsymbol{x}) & :=\int_{\mathbb{R}^{2}} \chi_{a, b}(|\boldsymbol{x}-\boldsymbol{y}|) \frac{1}{|\boldsymbol{x}-\boldsymbol{y}|} K_{2}(\boldsymbol{x}, \boldsymbol{y}) J(\boldsymbol{y}) \psi(\boldsymbol{y}) d \boldsymbol{y}, \quad \boldsymbol{x} \in \mathbb{R}^{2} .
\end{aligned}
$$

The kernel function of the global operator is smooth by assumption, which means that the smoothness is limited only by the smoothness of the cut off function $\chi_{a, b}$. Applying the quadrature scheme to the global operator we get the following operator

$$
\left(A_{\mathrm{g}, h} \psi\right)(\boldsymbol{x}):=Q_{h}^{2}\left[K_{\text {global }}(\boldsymbol{x}, \cdot) J \psi\right], \quad \boldsymbol{x} \in \mathbb{R}^{2},
$$

which we can write as the infinite sum

$$
\left(A_{\mathrm{g}, h} \psi\right)(\boldsymbol{x})=h^{2} \sum_{\boldsymbol{j} \in \mathbb{Z}^{2}} K_{\text {global }}(\boldsymbol{x}, h \boldsymbol{j}) J(h \boldsymbol{j}) \psi(h \boldsymbol{j}), \quad \boldsymbol{x} \in \mathbb{R}^{2} .
$$

For the treatment of the local operator (5.30) we introduce some additional notation. To simplify the understanding of the following rather technical definitions, we illustrated the situation in Figure 5.2. Let $\boldsymbol{j}_{\mathrm{ll}}^{\boldsymbol{x}}, \boldsymbol{j}_{\mathrm{ur}}^{\boldsymbol{x}} \in \mathbb{Z}^{2}$ denote the index of the lower left and upper right corner points of the smallest rectangle $R_{\boldsymbol{x}}$ in the grid that contains $B_{b}(\boldsymbol{x})$, an open ball of radius $b$ and centre $\boldsymbol{x}$. More formal, we define the two index sets

$$
J_{\text {ll }}^{\boldsymbol{x}}:=\left\{\boldsymbol{j} \in \mathbb{Z}^{2}: h \boldsymbol{j} \leq \boldsymbol{y} \text { for all } \boldsymbol{y} \in B_{b}(\boldsymbol{x})\right\}
$$

and

$$
J_{\mathrm{ur}}^{\boldsymbol{x}}:=\left\{\boldsymbol{j} \in \mathbb{Z}^{2}: \boldsymbol{y} \leq h \boldsymbol{j} \text { for all } \boldsymbol{y} \in B_{b}(\boldsymbol{x})\right\} .
$$

Then $\boldsymbol{j}_{11}^{\boldsymbol{x}} \in J_{\mathrm{ll}}^{\boldsymbol{x}}$ is the index such that $\boldsymbol{j} \leq \boldsymbol{j}_{\mathrm{ll}}^{\boldsymbol{x}}$ for all $\boldsymbol{j} \in J_{\mathrm{ll}}^{\boldsymbol{x}}$ and in an analogous fashion $\boldsymbol{j}_{\mathrm{ur}}^{\boldsymbol{x}} \in J_{\mathrm{ur}}^{\boldsymbol{x}}$ is the index such that $\boldsymbol{j}_{\mathrm{ll}}^{\boldsymbol{x}} \leq \boldsymbol{j}$ for all $\boldsymbol{j} \in J_{\mathrm{ur}}^{\boldsymbol{x}}$. Hence $R_{\boldsymbol{x}}=\left[h \boldsymbol{j}_{\mathrm{ll}}^{\boldsymbol{x}}, h \boldsymbol{j}_{\mathrm{ur}}^{\boldsymbol{x}}\right] \subset \mathbb{R}^{2}$. The function

$$
\Lambda_{\boldsymbol{x}}(\boldsymbol{y}):=\sqrt{\chi_{a, b}(|\boldsymbol{x}-\boldsymbol{y}|)} J(\boldsymbol{y}) \psi(\boldsymbol{y}), \quad \boldsymbol{y} \in \mathbb{R}^{2},
$$


has support in $B_{b}(\boldsymbol{x}) \subset R_{\boldsymbol{x}}$, so it possesses a uniquely determined periodic extension that coincides with $\Lambda_{\boldsymbol{x}}$ on $R_{\boldsymbol{x}}$. We approximate this periodic extension by the uniquely determined two dimensional trigonometric interpolation polynomial.

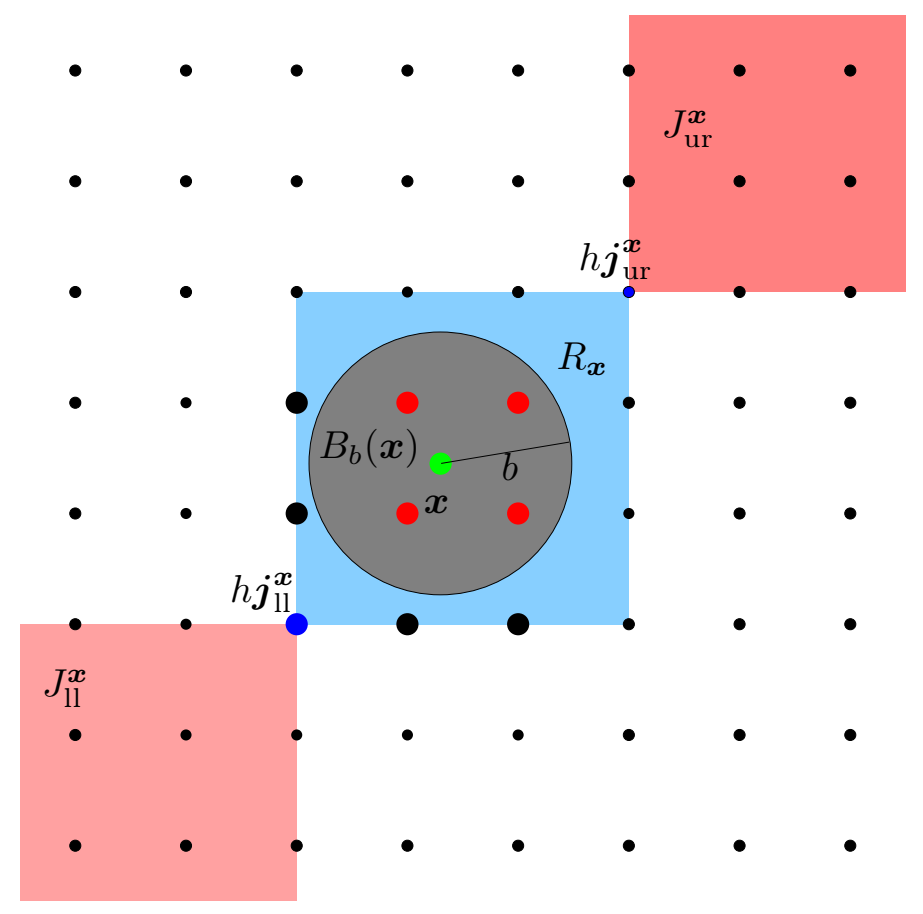

Figure 5.2: The setting for the local interpolation scheme: For a point $\boldsymbol{x}$ (green point), we depicted the circle $B_{b}(\boldsymbol{x})$ (gray) on which the function $\Lambda_{\boldsymbol{x}}$ is approximated. The grid points (red points), for which the locally corrected weights are computed, are the points inside the circle. The rectangle $R_{\boldsymbol{x}}$ is the blue square in the middle. It is determined by the lower left corner point $h \boldsymbol{j}_{11}^{\boldsymbol{x}}$ (enlarged blue point) and the upper right corner point $h \boldsymbol{j}_{\text {ur }}^{\boldsymbol{x}}$ (small blue point). The set of all enlarged points inside $R_{\boldsymbol{x}}$ are used for the interpolation.

For a function $\psi: R_{\boldsymbol{x}} \rightarrow \mathbb{C}$ we define the interpolation operator

$$
\left(P_{\boldsymbol{x}} \psi\right)(\boldsymbol{y}):=\sum_{\boldsymbol{j}_{11}^{\boldsymbol{x}} \leq \boldsymbol{j}<\boldsymbol{j}_{\mathrm{ur}}^{\boldsymbol{x}}} \psi(h \boldsymbol{j}) \ell_{\boldsymbol{j}-\boldsymbol{j}_{11}^{\boldsymbol{x}}}\left(\boldsymbol{y}-h \boldsymbol{j}_{\mathrm{ll}}^{\boldsymbol{x}}\right), \quad \boldsymbol{y} \in \mathbb{R}^{2},
$$

where $\ell_{\boldsymbol{j}}(\boldsymbol{y})$ for $0 \leq \boldsymbol{j}<\boldsymbol{L}$ denotes the Lagrange basis for the square $[0, h \boldsymbol{L}]$, where we have set $\boldsymbol{L}:=\boldsymbol{j}_{\mathrm{ur}}^{\boldsymbol{x}}-\boldsymbol{j}_{\mathrm{ll}}^{\boldsymbol{x}}$.

EXAMPLE 5.3. In the case that the number of points used for the trigonometric interpolation is even, i.e. $\boldsymbol{L}=(L, L) \in 2 \mathbb{Z}^{2}$, then the Lagrange basis is given through

$$
\ell_{\boldsymbol{j}}(\boldsymbol{y}):=\tilde{l}_{j_{1}}\left(\frac{\pi}{L h} y_{1}\right) \tilde{l}_{j_{2}}\left(\frac{\pi}{L h} y_{2}\right), \quad 0 \leq \boldsymbol{j} \leq \boldsymbol{L}-1,
$$


where

$$
\tilde{l}_{k}(t):=\frac{1}{L} \sin \left(\frac{L}{2}\left(t-\frac{\pi}{L} k\right)\right) \cot \frac{t-\frac{\pi}{L} k}{2}, \quad t \neq h k,
$$

for $k=0, \ldots, L-1$ denotes the one-dimensional Lagrange basis for the interval $[0,2 \pi]$, cf. [35, formula (11.13)].

We write the local operator in the form

$$
\left(A_{1} \psi\right)(\boldsymbol{x})=\int_{B_{b}(\boldsymbol{x})} \sqrt{\chi_{a, b}(|\boldsymbol{x}-\boldsymbol{y}|)} \frac{1}{|\boldsymbol{x}-\boldsymbol{y}|} K_{2}(\boldsymbol{x}, \boldsymbol{y}) \Lambda_{\boldsymbol{x}}(\boldsymbol{y}) d \boldsymbol{y},
$$

where $\Lambda_{\boldsymbol{x}}$ is given through (5.33). Replacing $\Lambda_{\boldsymbol{x}}$ by its trigonometric interpolation polynomial we define the operator

$$
\left(A_{l, h} \psi\right)(\boldsymbol{x}):=\int_{B_{b}(\boldsymbol{x})} \sqrt{\chi_{a, b}(|\boldsymbol{x}-\boldsymbol{y}|)} \frac{1}{|\boldsymbol{x}-\boldsymbol{y}|} K_{2}(\boldsymbol{x}, \boldsymbol{y})\left(P_{\boldsymbol{x}} \Lambda_{\boldsymbol{x}}\right)(\boldsymbol{y}) d \boldsymbol{y}, \quad \boldsymbol{x} \in \mathbb{R}^{2},
$$

which we can write in the form

$$
\left(A_{1, h} \psi\right)(\boldsymbol{x})=\sum_{\boldsymbol{j}_{\mathrm{ll}}^{\boldsymbol{x}} \leq \boldsymbol{j}<\boldsymbol{j}_{\mathrm{ur}}^{\boldsymbol{x}}} \tilde{\alpha}_{\boldsymbol{j}}(\boldsymbol{x}) J(h \boldsymbol{j}) \psi(h \boldsymbol{j}), \quad \boldsymbol{x} \in \mathbb{R}^{2},
$$

where

$$
\tilde{\alpha}_{\boldsymbol{j}}(\boldsymbol{x}):=\sqrt{\chi_{a, b}(|\boldsymbol{x}-h \boldsymbol{j}|)} \alpha_{\boldsymbol{j}}(\boldsymbol{x}), \quad \boldsymbol{j}_{\mathrm{ll}}^{\boldsymbol{x}} \leq \boldsymbol{j}<\boldsymbol{j}_{\mathrm{ur}}^{\boldsymbol{x}}
$$

and

$$
\alpha_{\boldsymbol{j}}(\boldsymbol{x}):=\int_{B_{b}(\boldsymbol{x})} \sqrt{\chi_{a, b}(|\boldsymbol{x}-\boldsymbol{y}|)} \frac{K_{2}(\boldsymbol{x}, \boldsymbol{y})}{|\boldsymbol{x}-\boldsymbol{y}|} \ell_{\boldsymbol{j}-\boldsymbol{j}_{11}^{x}}\left(\boldsymbol{y}-h \boldsymbol{j}_{11}^{\boldsymbol{x}}\right) d \boldsymbol{y}
$$

denote the locally corrected weights, which have to be computed by numerical integration. We set $\tilde{\alpha}_{\boldsymbol{j}}(\boldsymbol{x})=0$, for $\boldsymbol{j} \notin\left\{\boldsymbol{i} \in \mathbb{Z}^{2}: \boldsymbol{j}_{\mathrm{ll}}^{\boldsymbol{x}} \leq \boldsymbol{i}<\boldsymbol{j}_{\text {ur }}^{\boldsymbol{x}}\right\}$ to write (5.35) in the form

$$
\left(A_{1, h} \psi\right)(\boldsymbol{x}):=\sum_{j \in \mathbb{Z}^{2}} \tilde{\alpha}_{\boldsymbol{j}}(\boldsymbol{x}) J(h \boldsymbol{j}) \psi(h \boldsymbol{j}), \quad \boldsymbol{x} \in \mathbb{R}^{2},
$$

Using the function $p$ introduced above, cf. (5.9), we can write, after a change of variables,

$$
\alpha_{\boldsymbol{j}}(\boldsymbol{x})=\int_{0}^{b} \int_{0}^{2 \pi} \sqrt{\chi_{a, b}(r)} \tilde{K}_{2, \boldsymbol{x}}(r, \theta) \ell_{\boldsymbol{j}-\boldsymbol{j}_{11}^{x}}\left(\boldsymbol{x}+r p(\theta)-h \boldsymbol{j}_{\mathrm{ll}}^{\boldsymbol{x}}\right) d \theta d r .
$$

We observe that the singularity is completely removed through the Jacobian of this change of variables. The rather unmotivated use of the square root of the cut 
off function $\chi_{a, b}$ in the definition of $\Lambda_{\boldsymbol{x}}$ can now be justified. The assumption (W2) ensures that the integrand is a smooth function in the polar domain, whereas the cut off function allows us to interpret the integrand as a periodic function in the polar domain with $[-b, b] \times[0,2 \pi]$ as its domain of periodicity. Thus the application of the composite trapezoidal rule, both for the radial and angular direction, yields an efficient high order integration scheme.

The operator $A$ is approximated by

$$
\left(A_{h} \psi\right)(\boldsymbol{x}):=\left(A_{\mathrm{g}, h} \psi\right)(\boldsymbol{x})+\left(A_{1, h} \psi\right)(\boldsymbol{x}), \quad \boldsymbol{x} \in \mathbb{R}^{2},
$$

which again is an infinite sum of the form

$$
\left(A_{h} \psi\right)(\boldsymbol{x})=\sum_{\boldsymbol{j} \in \mathbb{Z}^{2}} K_{h, \boldsymbol{j}}(\boldsymbol{x}) J(h \boldsymbol{j}) \psi(h \boldsymbol{j}), \quad \boldsymbol{x} \in \mathbb{R}^{2},
$$

where

$$
K_{h, \boldsymbol{j}}(\boldsymbol{x}):=h^{2} K_{\text {global }}(\boldsymbol{x}, h \boldsymbol{j})+\tilde{\alpha}_{\boldsymbol{j}}(\boldsymbol{x}), \quad \boldsymbol{j} \in \mathbb{Z}^{2} .
$$

In analogy to the convergence results summarised in LEMMA 5.1, we prove a similar result for the weakly singular kernels that shows super-algebraic convergence.

Lemma 5.4. Let $A$ be given through (5.1), where $K: \mathbb{R}^{2} \times \mathbb{R}^{2} \rightarrow \mathbb{C}$ denotes a weakly singular kernel function that in addition satisfies the condition: there exists a constant $c>0$ such that

$$
\left|\partial^{\alpha} K(\boldsymbol{x}, \boldsymbol{y})\right| \leq \frac{c}{(1+|\boldsymbol{x}-\boldsymbol{y}|)^{2}} \quad \text { for } \quad|\boldsymbol{x}-\boldsymbol{y}| \geq 1
$$

for all $\alpha \in \mathbb{N}$ and the constant $c$ may depend on $\alpha$. Let $\psi \in B C_{q}^{\infty}\left(\mathbb{R}^{2}\right)$ with $q>0$ and $f \in B C^{\infty}\left(\mathbb{R}^{2}\right)$. Then, the operator $A_{h}$ for $h>0$ given through (5.2), with $K_{h, \boldsymbol{j}}$ given through (5.40), converges pointwise to $A$ and the error can be estimated through

$$
\left\|A \psi-A_{h} \psi\right\|_{B C\left(\mathbb{R}^{d}\right)} \leq\left\|A_{g} \psi-A_{g, h} \psi\right\|+\left\|A_{l} \psi-A_{l, h} \psi\right\|
$$

where

$$
\left\|A_{g} \psi-A_{g, h} \psi\right\| \leq C_{1}\left\|K_{\text {global }}(\boldsymbol{x}, \cdot)\right\|_{B C_{2}^{m}\left(\mathbb{R}^{2}\right)}\|J\|_{B C^{m}\left(\mathbb{R}^{2}\right)}\|\psi\|_{B C_{q}^{m}\left(\mathbb{R}^{2}\right)} h^{m},
$$

and

$$
\left\|A_{l} \psi-A_{l, h} \psi\right\| \leq C_{2}\left(\sup _{\boldsymbol{x} \in \mathbb{R}^{2}} \int_{B_{b}(\boldsymbol{x})}\left|K_{3}(\boldsymbol{x}, \boldsymbol{y})\right| d \boldsymbol{y}\right)\left\|\Lambda_{\boldsymbol{x}}-P_{\boldsymbol{x}} \Lambda_{\boldsymbol{x}}\right\|_{B C\left(\mathbb{R}^{2}\right)}
$$

with

$$
K_{3}(\boldsymbol{x}, \boldsymbol{y}):=\sqrt{\chi_{a, b}(|\boldsymbol{x}-\boldsymbol{y}|)} \frac{1}{|\boldsymbol{x}-\boldsymbol{y}|} K_{2}(\boldsymbol{x}, \boldsymbol{y})
$$

and the constants $C_{1}, C_{2}$ depend only on $m$ and $q$. 
Proof. The assumption (5.41) ensures that $K_{\text {global }} \in B C_{2}^{m}\left(\mathbb{R}^{2}\right)$ for all $m \geq 0$. Hence we can apply the same proof as in LEMMA 5.1 to get the estimate (5.42).

For the local operator we estimate

$$
\begin{aligned}
\left|A_{1} \psi(\boldsymbol{x})-A_{1, h}(\psi)(\boldsymbol{x})\right| & \leq \int_{B_{b}(\boldsymbol{x})}\left|K_{3}(\boldsymbol{x}, \boldsymbol{y})\right|\left|\Lambda_{\boldsymbol{x}}(\boldsymbol{y})-\left(P_{\boldsymbol{x}} \Lambda_{\boldsymbol{x}}\right)(\boldsymbol{y})\right| d \boldsymbol{y} \\
& \leq \sup _{\boldsymbol{x} \in R} \int_{B_{b}(\boldsymbol{x})}\left|K_{3}(\boldsymbol{x}, \boldsymbol{y})\right| d \boldsymbol{y}\left\|\Lambda_{\boldsymbol{x}}-\left(P_{\boldsymbol{x}} \Lambda_{\boldsymbol{x}}\right)\right\|_{B C\left(\mathbb{R}^{2}\right)} \\
& \leq C\left\|\Lambda_{\boldsymbol{x}}-\left(P_{\boldsymbol{x}} \Lambda_{\boldsymbol{x}}\right)\right\|_{B C\left(\mathbb{R}^{2}\right)}
\end{aligned}
$$

for some constant $C>0$ independent of $\boldsymbol{x}$. Now it follows from standard error estimates for smooth periodic functions that trigonometric interpolation will yield a super-algebraicly convergent scheme with respect to the mesh-size $h$ for a fixed cut-off radius $b$ and a smooth cut-off function $\chi_{a, b}$. The assumption on the weakly singular kernel, cf. LEMMA 1.15 for the details, ensure that

$$
\sup _{\boldsymbol{x} \in R} \int_{B_{b}(\boldsymbol{x})}\left|K_{3}(\boldsymbol{x}, \boldsymbol{y})\right| d \boldsymbol{y}
$$

is bounded on $\mathbb{R}^{2}$.

REMARK 5.5. The computational costs for the computations of the local corrected weights are the dominating costs in the overall scheme. We think it is therefore the best, to limit the numbers of points for which the local corrected weights are computed to a fixed number. A radius of $2 h$ or $3 h$, which means that 9 or 21 weights are being computed, seems to be a good choice. 


\section{Chapter 6}

\section{Nyström methods for rough surface scattering}

Using the parametrisation (1.8) of the scattering surface $\Gamma_{f}$ we can write the boundary integral equation (18) as an integral equation on $\mathbb{R}^{2}$. Setting

$$
\psi(\boldsymbol{x}):=\varphi(\boldsymbol{x}, f(\boldsymbol{x})) \text { and } \phi^{z}(\boldsymbol{x}):=-2 G((\boldsymbol{x}, f(\boldsymbol{x})), z)
$$

for some source point $z \in D_{f}$, we get an equation of the form

$$
\psi(\boldsymbol{x})+(W \psi)(\boldsymbol{x})=\phi^{z}(\boldsymbol{x}), \quad \boldsymbol{x} \in \mathbb{R}^{2},
$$

where $W$ denotes the integral operator

$$
(W \psi)(\boldsymbol{x}):=\int_{\mathbb{R}^{2}} k(\boldsymbol{x}, \boldsymbol{y}) J(\boldsymbol{y}) \psi(\boldsymbol{y}) d \boldsymbol{y}, \quad \boldsymbol{x} \in \mathbb{R}^{2}
$$

with kernel function

$$
k(\boldsymbol{x}, \boldsymbol{y}):=2\left\{\frac{G(x, y)}{\partial \nu(y)}-i \eta 2 G(x, y)\right\}, \quad x \neq y,
$$

for $x=(\boldsymbol{x}, f(\boldsymbol{x})), y=(\boldsymbol{y}, f(\boldsymbol{y}))$ and surface area element $J(\boldsymbol{y})=\sqrt{1+|\nabla f(\boldsymbol{y})|^{2}}$. Introducing the operators

$$
\left(W^{\xi} \psi\right)(\boldsymbol{x}):=\int_{\mathbb{R}^{2}} k^{\xi}(\boldsymbol{x}, \boldsymbol{y}) J(\boldsymbol{y}) \psi(\boldsymbol{y}) d \boldsymbol{y}, \quad \boldsymbol{x} \in \mathbb{R}^{2},
$$

for $\xi \in\{S, K\}$ with kernel functions $k^{S}$ and $k^{K}$ given through (5.6) and (5.7), we can write the operator (6.3) in the form

$$
W=W^{K}-i \eta W^{S}
$$

We approximate both operators $W^{K}$ and $W^{S}$ as described in the previous chapter, i.e. the integral operator $W$ is approximated by

$$
\left(W_{h} \psi\right)(\boldsymbol{x}):=\sum_{\boldsymbol{j} \in \mathbb{Z}^{2}} k_{h, \boldsymbol{j}}(\boldsymbol{x}) J(h \boldsymbol{j}) \psi(h \boldsymbol{j}), \quad \boldsymbol{x} \in \mathbb{R}^{2},
$$


where

$$
k_{h, \boldsymbol{j}}(\boldsymbol{x}):=\left[h^{2} k_{\text {global }}^{K}(\boldsymbol{x}, h \boldsymbol{j})-i \eta h^{2} k_{\text {global }}^{S}(\boldsymbol{x}, h \boldsymbol{j})\right]+\left[\tilde{\alpha}_{\boldsymbol{j}}^{K}(\boldsymbol{x})-i \eta \tilde{\alpha}_{\boldsymbol{j}}^{S}(\boldsymbol{x})\right] .
$$

Now one approximates the solution of equation (6.2) by the solution of the equation

$$
\psi_{h}(\boldsymbol{x})+\left(W_{h} \psi_{h}\right)(\boldsymbol{x})=\phi^{z}(\boldsymbol{x}), \quad \boldsymbol{x} \in \mathbb{R}^{2} .
$$

In complete analogy to the Nyström method for the case of finite intervals one proves the following theorem.

LEMma 6.1. For some $h>0$ let $\psi_{h}$ be a solution of

$$
\psi_{h}(\boldsymbol{x})+\sum_{\boldsymbol{j} \in \mathbb{Z}^{2}} k_{h, \boldsymbol{j}}(\boldsymbol{x}) J(h \boldsymbol{j}) \psi_{h}(h \boldsymbol{j})=\phi^{z}(\boldsymbol{x}), \quad \boldsymbol{x} \in \mathbb{R}^{2} .
$$

Then the sequence $\psi^{h}=\left(\psi_{\boldsymbol{j}}^{h}\right)_{\boldsymbol{j} \in \mathbb{Z}^{2}}$ with $\psi_{\boldsymbol{j}}^{h}:=\psi_{h}(h \boldsymbol{j}), \boldsymbol{j} \in \mathbb{Z}^{2}$ solves the infinite set of equations

$$
\psi_{\boldsymbol{i}}^{h}+\sum_{\boldsymbol{j} \in \mathbb{Z}^{2}} k_{h, \boldsymbol{j}}(h \boldsymbol{i}) J(h \boldsymbol{j}) \psi_{\boldsymbol{j}}^{h}=\phi^{z}(h \boldsymbol{i}), \quad \boldsymbol{i} \in \mathbb{Z}^{2} .
$$

Conversely, if the sequence $\psi^{h}=\left(\psi_{\boldsymbol{j}}^{h}\right)_{\boldsymbol{j} \in \mathbb{Z}^{2}}$ is a solution of (6.9), we get a solution of (6.8) through

$$
\psi_{h}(\boldsymbol{x}):=\phi^{z}(\boldsymbol{x})-\sum_{\boldsymbol{j} \in \mathbb{Z}^{2}} k_{h, \boldsymbol{j}}(\boldsymbol{x}) J(h \boldsymbol{j}) \psi_{\boldsymbol{j}}^{h}, \quad \boldsymbol{x} \in \mathbb{R}^{2}
$$

that agrees with the sequence $\left(\psi_{\boldsymbol{j}}^{h}\right)_{\boldsymbol{j} \in \mathbb{Z}^{2}}$ at the set of quadrature points. This interpolation function is called Nyström interpolant.

Proof. The first part is trivial. For the second part one can argue as follows. For a sequence $\left(\psi_{\boldsymbol{j}}^{h}\right)_{\boldsymbol{j} \in \mathbb{Z}^{2}}$ that solves $(6.9)$ we see that the function $\psi_{h}$, given through (6.10), takes the values

$$
\psi_{h}(h \boldsymbol{i})=\phi^{z}(h \boldsymbol{i})-\sum_{\boldsymbol{j} \in \mathbb{Z}^{2}} k_{h, \boldsymbol{j}}(h \boldsymbol{i}) J(h \boldsymbol{j}) \psi_{\boldsymbol{j}}^{h}=\psi_{\boldsymbol{i}}^{h}, \quad \boldsymbol{i} \in \mathbb{Z}^{2} .
$$

Inserting this, together with (6.10), into (6.8) shows that $\psi_{h}$ solves the equation (6.8)

REMARK 6.2. The system (6.9) can be written as a linear equation in $\ell^{2}\left(\mathbb{Z}^{2}\right)$, i.e.

$$
\psi^{h}+\widehat{W}_{h} \psi^{h}=\phi^{z, h},
$$

where $\phi^{z, h}=\left(\phi_{\boldsymbol{j}}^{z, h}\right)_{\boldsymbol{j} \in \mathbb{Z}^{2}}$ with $\phi_{\boldsymbol{j}}^{z, h}:=\phi^{z}(h \boldsymbol{j})$ for $\boldsymbol{j} \in \mathbb{Z}^{2}$ and where we have introduced the operator

$$
\widehat{W}_{h}: \ell^{2}\left(\mathbb{Z}^{2}\right) \rightarrow \ell^{2}\left(\mathbb{Z}^{2}\right), \quad \psi \mapsto\left(\sum_{\boldsymbol{j} \in \mathbb{Z}^{2}} k_{h, \boldsymbol{j}}(h \boldsymbol{i}) J(h \boldsymbol{j}) \psi_{\boldsymbol{j}}\right)_{\boldsymbol{i} \in \mathbb{Z}^{2}} .
$$


Though it is clear that we can never implement this infinite linear system on a computer, the approach is interesting from a theoretical point of view. In the case of scattering by one-dimensional rough surfaces, some results on the convergence of the Nyström method were proven in [2], [40] and [41].

\subsection{Method I: Discretisation-truncation}

To get a finite dimensional linear system that we can actually implement on a computer we restrict the quadrature points to the finite set

$$
\{h \boldsymbol{j}:-N \leq \boldsymbol{j} \leq N-1\}
$$

where we have set

$$
N:=(n, n) \in \mathbb{N}^{2}
$$

for some $n \in \mathbb{N}$. Thus instead of using the solution of (6.8) as an approximation to the true solution, we approximate the solution of (6.2) by the solution of

$$
\psi_{h, \varrho}(\boldsymbol{x})+\left(W_{h, \varrho} \psi_{h, \varrho}\right)(\boldsymbol{x})=\phi^{z}(\boldsymbol{x}), \quad \boldsymbol{x} \in \mathbb{R}^{2} .
$$

where we have set

$$
\varrho:=h \cdot n
$$

and

$$
\left(W_{h, \varrho} \psi\right)(\boldsymbol{x}):=\sum_{\boldsymbol{j}=-N}^{N-1} k_{h, \boldsymbol{j}}(\boldsymbol{x}) J(h \boldsymbol{j}) \psi(h \boldsymbol{j}), \quad \boldsymbol{x} \in \mathbb{R}^{2} .
$$

The meaning of the parameter $\varrho$ will become clear in the next section. In the same manner as before one proves the following theorem.

Lemma 6.3. For some $h>0$ and $n \in \mathbb{N}$ let $\psi_{h, \varrho}$ be a solution of

$$
\psi_{h, \varrho}(\boldsymbol{x})+\sum_{\boldsymbol{j}=-N}^{N-1} k_{h, \boldsymbol{j}}(\boldsymbol{x}) J(h \boldsymbol{j}) \psi_{h, \varrho}(h \boldsymbol{j})=\phi^{z}(\boldsymbol{x}), \quad \boldsymbol{x} \in \mathbb{R}^{2},
$$

where $N$ and $\varrho$ are given through (6.13) and (6.14). Then the two-dimensional array $\boldsymbol{\psi}=\left(\psi_{\boldsymbol{j}}\right)_{\boldsymbol{j}=-N, \ldots, N-1} \in \mathbb{C}^{\times(2 N)}$ with $\psi_{\boldsymbol{j}}:=\psi_{h, \varrho}(h \boldsymbol{j})$ for $\boldsymbol{j}=-N, \ldots, N-1$ solves the finite dimensional system

$$
\psi_{\boldsymbol{i}}+\sum_{\boldsymbol{j}=-N}^{N-1} k_{h, \boldsymbol{j}}(h \boldsymbol{i}) J(h \boldsymbol{j}) \psi_{\boldsymbol{j}}=\phi^{z}(h \boldsymbol{i}), \quad \boldsymbol{i}=-N, \ldots, N-1
$$


Conversely, if the array $\boldsymbol{\psi}=\left(\psi_{\boldsymbol{j}}\right)_{\boldsymbol{j}=-N, \ldots, N-1}$ is a solution of $(6.17)$, we get a solution of (6.16) through

$$
\psi_{h, \varrho}(\boldsymbol{x}):=\phi^{z}(\boldsymbol{x})-\sum_{\boldsymbol{j}=-N}^{N-1} k_{h, \boldsymbol{j}}(\boldsymbol{x}) J(h \boldsymbol{j}) \psi_{\boldsymbol{j}}, \quad \boldsymbol{x} \in \mathbb{R}^{2} .
$$

REMARK 6.4. We note that the linear system (6.17) is simply a truncated version of the infinite linear system (6.9).

\subsection{Method II: Truncation-discretisation}

A different way to arrive at this system is the following. For a given step size $h>0$ and $n \in \mathbb{N}$ we consider the truncated version of (6.2), namely

$$
\psi_{\varrho}(\boldsymbol{x})+\left(W_{\varrho} \psi_{\varrho}\right)(\boldsymbol{x})=\phi^{z}(\boldsymbol{x}), \quad \boldsymbol{x} \in[-\varrho, \varrho]^{2},
$$

where $\varrho$ is given through $(6.14)$ and

$$
\left(W_{\varrho} \psi\right)(\boldsymbol{x}):=\int_{[-\varrho, \varrho]^{2}} k(\boldsymbol{x}, \boldsymbol{y}) J(\boldsymbol{y}) \psi(\boldsymbol{y}) d \boldsymbol{y}, \quad \boldsymbol{x} \in[-\varrho, \varrho]^{2},
$$

as a starting point for our calculations. Now we apply a Nyström method, where we are using the same operator approximations as before. Using the integration points given through (6.12) yields the equation

$$
\psi_{\varrho, h}(\boldsymbol{x})+\left(W_{\varrho, h} \psi_{\varrho, h}\right)(\boldsymbol{x})=\phi^{z}(\boldsymbol{x}), \quad \boldsymbol{x} \in[-\varrho, \varrho]^{2},
$$

where

$$
\left(W_{\varrho, h} \psi\right)(\boldsymbol{x}):=\sum_{\boldsymbol{j}=-N}^{N-1} k_{h, \boldsymbol{j}}(\boldsymbol{x}) J(h \boldsymbol{j}) \psi(h \boldsymbol{j}), \quad \boldsymbol{x} \in[-\varrho, \varrho]^{2}
$$

and $N$ is given through (6.13). To get an approximate solution of (6.19), we see that it suffices to solve the linear system (6.16).

Thus we see that, due to the unboundedness of the integration domain, the numerical schemes to solve the integral equation (6.2) require an additional approximation step. This truncation step is called finite section method and we give a slightly more formal treatment in the next section. As we demonstrated above it can be realised in two slightly different ways:

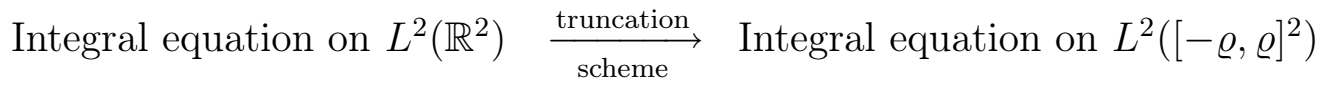

$$
\begin{aligned}
& \downarrow \text { discretisation } \downarrow \quad \downarrow \text { discretisation } \downarrow
\end{aligned}
$$$$
\text { Linear equation on } \ell^{2}\left(\mathbb{Z}^{2}, \mathbb{C}\right) \underset{\text { scheme }}{\stackrel{\text { truncation }}{\longrightarrow}} \text { Linear system on } \mathbb{C}^{(2 N)^{2}} \text {. }
$$ 
These two view points explain e.g. why the trapezoidal rule is a good choice for a quadrature rule. If one first truncates the integral equation one might object that a higher order quadrature rule, like e.g. the composite Simpson rule, would be more appropriate for the discretisation. But we are looking for a quadrature rule that converges to high order to the untruncated version of the operator. Hence, one should use a quadrature rule that gives equal weights to every quadrature point.

\subsection{The Finite Section Method}

The truncation scheme that we presented is known under the name finite section method. Let us consider the following prototype equation

$$
A \varphi=f
$$

where $A \in G L(Y) \subset B L(Y)$ is an invertible operator on some Banach space $Y$. In the context of the scattering problem $A$ is given through $I+W$, the Banach space $Y$ stands for any of the spaces $L^{2}\left(\mathbb{R}^{2}\right), X\left(\mathbb{R}^{2}\right)$ or $X_{\infty}\left(\mathbb{R}^{2}\right)$ and $f$ stands for the right hand side $\phi^{z}$.

Then, the finite section method consists in replacing (6.23) by

$$
P_{\varrho} A P_{\varrho} \varphi_{\varrho}=P_{\varrho} f
$$

where $\varrho>0$ and the operator $P_{\varrho}: Y \rightarrow Y$, is given by

$$
\left(P_{\varrho} \psi\right)(x):= \begin{cases}\psi(x), & |x|<\varrho \\ 0, & \text { otherwise. }\end{cases}
$$

Here, $|\cdot|$ denotes any norm on $\mathbb{R}^{2}$. In the case that $|\cdot|=\|\cdot\|_{\infty}$, we see that $(6.24)$ coincides with (6.19) in the case of the scattering problem. Provided equation (6.23) is uniquely solvable for every right-hand side one hopes that also equation (6.24) is uniquely solvable considered as an equation in $L^{2}\left(B_{\varrho}\right) \subset L^{2}\left(\mathbb{R}^{2}\right)$, where

$$
B_{\varrho}:=\left\{\boldsymbol{x} \in \mathbb{R}^{2}:|\boldsymbol{x}|<1\right\},
$$

and that its solution $\varphi_{\varrho}$ approximates the exact solution $\varphi$ of (6.23) if only one chooses $\varrho$ large enough. If this is the case, then this method is called applicable.

For recent results on the applicability of the finite section method for the fairly large class of all so-called band-dominated operators in terms of their limit operators see e.g. [38], [47] and the references therein. The operators originating from 2D rough surface scattering are band-dominated, and we refer to [40], [41] and [13] and further literature cited therein for the study and application of the finite section method to these equations. As we pointed it out before, the applicability of the finite section method to the equation (6.2) is to the author's understanding still an open problem. 
We underline that the framework for the FSM not only covers the set of banddominated operators, it even applies to the much larger class, called $L(Y, \mathcal{P})$ in [38] and [47], that consists of all bounded linear operators $A$ on $Y$ for which

$$
\left\|\left(I-P_{\varrho}\right) A P_{\tau}\right\| \rightarrow 0 \text { and } \quad\left\|P_{\tau} A\left(I-P_{\varrho}\right)\right\| \rightarrow 0 \quad \text { as } \quad \varrho \rightarrow \infty
$$

for every fixed $\tau>0$.

The multi-section method (MSM) that we present in some more detail in Chapter 7 , requires only the first condition in (6.27) to be true.

\subsection{The Banded Matrix Iterative Algorithm for rough surface scattering}

We have seen in the previous sections that the numerical solution of (6.2) with the help of a Nyström method in combination with the finite section method, yields a finite dimensional linear system that can be written as

$$
\psi+\boldsymbol{L} \psi+\boldsymbol{G} \psi=\phi^{z}
$$

where $\boldsymbol{L}, \boldsymbol{G} \in \mathbb{C}^{[\times(2 N)] \times[\times(2 N)]}$ denote two-dimensional matrices with

$$
\begin{aligned}
(\boldsymbol{L})_{\boldsymbol{i}, \boldsymbol{j}} & :=\tilde{\alpha}_{\boldsymbol{j}}^{K}(h \boldsymbol{i}) J(h \boldsymbol{j})-i \eta \tilde{\alpha}_{\boldsymbol{j}}^{S}(h \boldsymbol{i}) J(h \boldsymbol{j}), \\
(\boldsymbol{G})_{\boldsymbol{i}, \boldsymbol{j}} & :=h^{2} k_{\text {global }}^{K}(h \boldsymbol{i}, h \boldsymbol{j}) J(h \boldsymbol{j})-i \eta h^{2} k_{\text {global }}^{S}(h \boldsymbol{i}, h \boldsymbol{j}) J(h \boldsymbol{j}),
\end{aligned}
$$

for $-N \leq \boldsymbol{i}, \boldsymbol{j} \leq N-1$ and $\boldsymbol{\psi}, \boldsymbol{\phi}^{z} \in \mathbb{C}^{\times(2 N)}$ denote two-dimensional arrays with

$$
\begin{aligned}
\boldsymbol{\psi}_{\boldsymbol{j}} & :=\psi(h \boldsymbol{j}), \\
\boldsymbol{\phi}_{\boldsymbol{j}}^{z} & :=\phi^{z}(h \boldsymbol{j}),
\end{aligned}
$$

for $-N \leq \boldsymbol{j} \leq N-1$.

The matrix $\boldsymbol{L}$ is a sparse matrix with $O(\sharp(2 N))$ nonzero entries with band structure containing the locally corrected weights and the matrix $\boldsymbol{G}$ is a dense matrix containing the smooth global part of the integral operators.

To solve the large and dense linear system (6.28) it was suggested to employ an iterative algorithm that, in the engineering literature, has been termed banded matrix iterative algorithm (BMIA). The main idea is to use a decomposition of the system matrix into a sparse banded matrix with finite band-width and a large dense matrix. We claim that the natural decomposition given in (6.28) can be applied, i.e. in this case the algorithm consists in computing the expressions

$$
\begin{aligned}
\boldsymbol{\psi}^{(0)} & :=\mathbf{0} \\
\boldsymbol{\psi}^{(n+1)} & :=(\boldsymbol{I}+\boldsymbol{L})^{-1} \cdot\left(\boldsymbol{\phi}^{z}-\boldsymbol{G} \cdot \boldsymbol{\psi}^{(n)}\right), \quad n=0,1,2, \ldots
\end{aligned}
$$


Thus in each step one has to solve a linear system with a sparse banded matrix $\boldsymbol{I}+\boldsymbol{L}$ and compute one matrix-vector multiplication with a dense unstructured matrix $\boldsymbol{G}$. The matrix $\boldsymbol{I}+\boldsymbol{L}$ is actually a block band matrix with band blocks. Therefore, to solve this linear system in each step it is again reasonable to use an iterative scheme, e.g. the GMRES. The most time consuming step however is the computation of the matrix-vector product $\boldsymbol{G} \cdot \boldsymbol{\psi}^{(n)}$. It is possible to design fast matrix-vector multiplications for this product by approximating the matrix $\boldsymbol{G}$ in a suitable manner. These approximations are examined in Chapter 9.

We need to point out that we do not know whether this decomposition is feasible, i.e. we do not know whether $\boldsymbol{I}+\boldsymbol{L}$ is always invertible. First numerical tests show that the system is indeed invertible and well conditioned.

For more results on the convergence of a slight variation of this method we refer the reader to [6] for the case of $2 \mathrm{D}$ rough surface scattering problems.

Just like for any iterative algorithm there is the question when to stop the iterations. As a replacement for the error $\left\|\boldsymbol{\psi}-\boldsymbol{\psi}^{(n)}\right\|$ one uses the residual

$$
\text { res }:=\left\|\phi^{z}-(\boldsymbol{I}+\boldsymbol{L}+\boldsymbol{G}) \cdot \boldsymbol{\psi}^{(n+1)}\right\|
$$

and stops the iteration, once the residual is smaller than a given tolerance Tol. It follows from

$$
\begin{aligned}
\boldsymbol{\phi}^{z}-(\boldsymbol{I}+\boldsymbol{L}+\boldsymbol{G}) \cdot \boldsymbol{\psi}^{(n+1)}= & \boldsymbol{\phi}^{z}-(\boldsymbol{I}+\boldsymbol{L}+\boldsymbol{G}) \cdot\left[(\boldsymbol{I}+\boldsymbol{L})^{-1} \cdot\left(\boldsymbol{\phi}^{z}-\boldsymbol{G} \cdot \boldsymbol{\psi}^{(n)}\right)\right] \\
= & \boldsymbol{\phi}^{z}-(\boldsymbol{I}+\boldsymbol{L}) \cdot(\boldsymbol{I}+\boldsymbol{L})^{-1} \cdot\left(\boldsymbol{\phi}^{z}-\boldsymbol{G} \cdot \boldsymbol{\psi}^{(n)}\right) \\
& \quad-\boldsymbol{G} \cdot(\boldsymbol{I}+\boldsymbol{L})^{-1} \cdot\left(\boldsymbol{\phi}^{z}-\boldsymbol{G} \cdot \boldsymbol{\psi}^{(n)}\right) \\
= & \boldsymbol{G} \cdot \boldsymbol{\psi}^{(n)}-\boldsymbol{G} \cdot \boldsymbol{\psi}^{(n+1)}
\end{aligned}
$$

that

$$
\text { res }=\left\|\boldsymbol{G} \cdot \boldsymbol{\psi}^{(n+1)}-\boldsymbol{G} \cdot \boldsymbol{\psi}^{(n)}\right\| .
$$

The algorithm with stopping rule is given in pseudo code in Algorithm 6.1. 
ALGORITHM 6.1 The banded matrix iterative algorithm in pseudo code

\section{Input data:}

$\phi^{z} \in \mathbb{C}^{\times(2 N)}$

$\boldsymbol{L} \in \mathbb{C}^{[\times(2 N)] \times[\times(2 N)]}$

$\boldsymbol{G} \in \mathbb{C}^{[\times(2 N)] \times[\times(2 N)]}$

$\%$ right hand side

Tol

$\%$ sparse banded block band matrix

$\%$ dense matrix

$\%$ tolerance

\section{Initialisation:}

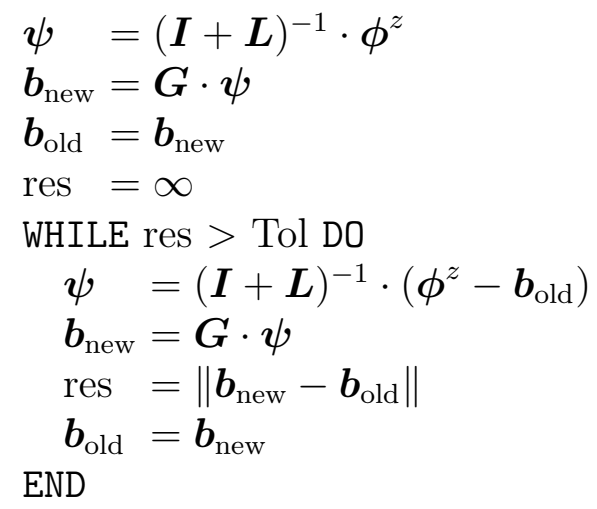

\section{Output:}




\section{Chapter 7}

\section{The Multi-Section Method}

A generalisation to the finite section method has been proposed in [31], which we termed multi-section method. For this scheme we can prove the applicability to the integral equation (6.2).

For a general framework we consider the following setting: Let $Y$ be a Banach space and let $\left\{P_{\varrho}\right\}_{\varrho>0}$ be a family of linear operators on $Y$ with the following three properties,

(P1) $P_{\varrho} P_{\tau}=P_{\tau}=P_{\tau} P_{\varrho}$ for all $\varrho \geq \tau>0$,

(P2) $\left\|P_{\varrho}\right\|=1$ for all $\varrho>0$,

(P3) $P_{\varrho} \rightarrow I$ pointwise, that means $P_{\varrho} \varphi \rightarrow \varphi$ for all $\varphi \in Y$, as $\varrho \rightarrow \infty$.

From $(\mathbf{P} 1)$, with $\varrho=\tau$, we conclude that every $P_{\varrho}$ is a projection operator. We will also have to deal with the complementary projectors

$$
Q_{\varrho}:=I-P_{\varrho}, \quad \varrho>0 .
$$

Now suppose $A$ is a bounded linear operator on $Y$ such that

(A1) $A$ is invertible, and therefore boundedly invertible, on $Y$,

(A2) $\left\|Q_{\varrho} A P_{\tau}\right\| \rightarrow 0$ as $\varrho \rightarrow \infty$ for every fixed $\tau>0$.

To find an approximation to the solution of the equation

$$
A \varphi=f
$$

we proposed the following method.

Definition 7.1 (Multi-section method (MSM)). For given precision $\delta>0$ and sufficiently large cut-off parameters $\varrho$ and $\tau$, calculate a solution $\psi \in Y$ of the system

$$
\begin{cases}P_{\tau} \psi & =\psi, \\ \left\|P_{\varrho} A P_{\tau} \psi-P_{\varrho} f\right\|_{Y} & \leq \delta .\end{cases}
$$


If applied to equation (6.2) it means that, in contrast to exactly solving the truncated equation (6.19) for large $\varrho$, we look for a function $\psi \in Y$ that has support in $B_{\tau}$ and approximately solves the truncated equation

$$
P_{\varrho}(I+W) P_{\tau} \psi \approx P_{\varrho} \phi^{z}
$$

for large $\varrho, \tau$ and a given discrepancy allowance $\delta$ in the ' $\approx$ ' sign.

So the two main differences to the finite section method are:

(a) We allow two different cut-off parameters $\varrho$ and $\tau$ instead of just one.

(b) We work with approximate instead of exact solutions.

REMARK 7.2. Point (a) is the reason this method was termed multi-section method. From the matrix perspective it means that we cut rectangular rather than quadratic finite matrices out of the original infinite matrix that represents the discretised form of the operator $I+W$ in the space $\ell^{2}\left(\mathbb{Z}^{2}, \mathbb{C}\right)$, cf. (6.11).

\subsection{Existence and convergence}

The main results that we showed in [31] concern the existence of multi-section solutions and the convergence of the multi-section method.

To cite the results from [31], we introduce the following useful definition.

DEFINITION 7.3. We say that $\tau_{0}>0$ is an admissible $\tau$-bound for a given precision $\delta>0$ if (7.3) is solvable in $Y$ for all $\varrho>0$ and $\tau>\tau_{0}$.

Let $A$ be a linear operator on a Banach space $Y$ that satisfies the conditions (A1) and (A2), then the following holds:

TheOrem 7.4 (Existence of solutions to (7.3) (MSM)). For every $\delta>0$, there is an admissible $\tau$-bound $\tau_{0}=\tau_{0}(\delta)>0$.

Proof. See [31, Theorem 3.8].

TheOREm 7.5 (Convergence of the Multi-Section Method). For every $\varepsilon>0$, there are parameters $\delta, \varrho, \tau$ such that every solution $\psi \in Y$ of the system (7.3) is an approximation of the exact solution $\varphi$ of (7.2), i.e.

$$
\|\varphi-\psi\|_{Y}<\varepsilon \text {. }
$$

Precisely, there are functions $\delta_{0}, \tau_{0}: \mathbb{R}_{+} \rightarrow \mathbb{R}_{+}$and $\varrho_{0}: \mathbb{R}_{+}^{3} \rightarrow \mathbb{R}_{+}$such that, if $\delta<\delta_{0}(\varepsilon), \tau>\tau_{0}(\delta)$ and $\varrho>\varrho_{0}(\varepsilon, \delta, \tau)$, then every solution $\psi \in Y$ of $(7.3)$ is subject to $(7.5)$.

Proof. See [31, Theorem 3.10]. 


\subsection{Applicability to the rough surface scattering problem}

We illustrate the applicability of the MSM to the following general example of an integral equation of the second kind that includes the operators arising from the rough surface scattering problem.

Lemma 7.6. Let $Y=L^{p}\left(\mathbb{R}^{n}\right)$ with $1 \leq p<\infty, n \in \mathbb{N}$ and let $A \in G L(Y)$, the set of all boundedly invertible operators on $Y$, such that $A=I+W$, where $W$ is a well-defined and bounded integral operator

$$
(W \varphi)(x)=\int_{\mathbb{R}^{n}} k(x, y) \varphi(y) d y, \quad x \in \mathbb{R}^{n}
$$

on $Y$ with a kernel function that satisfy a decay condition

$$
|k(x, y)| \leq \frac{C}{|x-y|^{\gamma}} \quad \text { for } \quad|x-y|>1
$$

with constants $\gamma>0$ and some $C>0$. Furthermore let $\left\{P_{\varrho}\right\}_{\varrho>0}$ denote the family of operators given through (6.25). Then the MSM is convergent in the sense of THEOREM 7.5 if $\gamma p>n$.

Proof. To prove this result, we note that $Y$ is a Banach space and the family $\left\{P_{\varrho}\right\}_{\varrho>0}$ is clearly subject to the assumptions $(\mathbf{P 1})-(\mathbf{P} 3)$. Thus we are left to show that (A2) holds.

We prove that, for every $\tau>0$, we have

$$
\left\|Q_{\varrho} A P_{\tau}\right\|_{L^{p}\left(\mathbb{R}^{n}\right)} \leq \frac{c}{\varrho^{\gamma-n / p}}, \quad \varrho>2 \tau
$$

with some constant $c>0$ depending on $\tau$. In particular we see that, if $\gamma p>n$, assumption (A2) holds.

Let $B_{\varrho}=\left\{x \in \mathbb{R}^{n}:|x|<\varrho\right\}$ be the ball and $\partial B_{\varrho}=\left\{x \in \mathbb{R}^{n}:|x|=\varrho\right\}$ the sphere of radius $\varrho>0$ in $\mathbb{R}^{n}$, and denote their $n$ - and $(n-1)$-dimensional measure by $\left|B_{\varrho}\right|$ and $\left|\partial B_{\varrho}\right|$, respectively. Now take some $\varrho>2 \tau>0$ and first suppose $1<p<\infty$. 
Using Hölder's inequality with $1 / p+1 / q=1$ we get the following:

$$
\begin{aligned}
\left\|Q_{\varrho} A P_{\tau} \varphi\right\|_{L^{p}\left(\mathbb{R}^{n}\right)}^{p} & =\left\|Q_{\varrho} I P_{\tau} \varphi+Q_{\varrho} W P_{\tau} \varphi\right\|_{L^{p}\left(\mathbb{R}^{n}\right)}^{p}=\left\|Q_{\varrho} W P_{\tau} \varphi\right\|_{L^{p}\left(\mathbb{R}^{n}\right)}^{p} \\
& =\int_{|x| \geq \varrho}\left|\left(W P_{\tau} \varphi\right)(x)\right|^{p} d x \\
& =\int_{|x| \geq \varrho}\left|\int_{|y|<\tau} k(x, y) \varphi(y) d y\right|^{p} d x \\
& \leq \int_{|x| \geq \varrho}\left(\left(\int_{|y|<\tau}|k(x, y)|^{q} d y\right)^{1 / q} \cdot\left\|P_{\tau} \varphi\right\|_{L^{p}\left(\mathbb{R}^{n}\right)}\right)^{p} d x \\
& \leq \int_{|x| \geq \varrho}\left(\int_{|y|<\tau}|k(x, y)|^{q} d y\right)^{p / q} d x \cdot\|\varphi\|_{L^{p}\left(\mathbb{R}^{n}\right)}^{p}
\end{aligned}
$$

Consequently, using the bound (7.7) and the inequality

$$
|x| \geq \varrho>2 \tau>2|y|, \quad \text { which implies } \quad|x-y| \geq|x|-|y|>|x| / 2,
$$

we get

$$
\begin{aligned}
\left\|Q_{\varrho} A P_{\tau}\right\|^{p} & \leq \int_{|x| \geq \varrho}\left(\int_{|y|<\tau} \frac{C^{q}}{|x-y|^{\gamma q}} d y\right)^{p / q} d x \\
& \leq \int_{|x| \geq \varrho}\left(\int_{|y|<\tau} \frac{C^{q}}{(|x| / 2)^{\gamma q}} d y\right)^{p / q} d x \\
& =\left(\int_{|x| \geq \varrho} \frac{1}{|x|^{\gamma p}} d x\right)\left(\int_{|y|<\tau} 1 d y\right)^{p / q}\left(2^{\gamma} C\right)^{p} \\
& =\left(\int_{r=\varrho}^{\infty} \frac{1}{r^{\gamma p}}\left|\partial B_{r}\right| d r\right)\left|B_{\tau}\right|^{p / q}\left(2^{\gamma} C\right)^{p} \\
& =\left(\int_{r=\varrho}^{\infty} \frac{r^{n-1}}{r^{\gamma p}} d r\right)\left|\partial B_{1}\right|\left|B_{\tau}\right|^{p / q}\left(2^{\gamma} C\right)^{p} \\
& =\varrho^{n-\gamma p} \frac{1}{\gamma p-n}\left|\partial B_{1}\right|\left|B_{\tau}\right|^{p / q}\left(2^{\gamma} C\right)^{p} .
\end{aligned}
$$

Finally, taking $p$-th roots proves (7.8). The proof for $p=1$ is similar. But instead of using Hölder's inequality one immediately arrives at (7.9), with $p / q$ replaced by 0 .

REMARK 7.7. Note that this example, with $p=n=\gamma=2$, covers the integral equation (6.2) arising from the boundary integral formulation of $3 D$ rough surface scattering problems as discussed before.

Thus we conclude the following theorem (cf. [31, Theorem 3.12]). 
THEOREM 7.8. The multi section method, as defined in DeFINITION 7.1, applied to the integral equation (6.2) is convergent in the sense of THEOREM 7.5.

\subsection{Numerical realisation}

The goal of this section is to provide a numerical algorithm for the solution of the integral equation (6.2) using the MSM.

Our suggestion for a numerical approach to the solution of the system (7.3) for the concrete operator equation (6.2) is to choose some large parameters $\varrho>\tau$ and to choose the discrepancy $\delta$ as small as it possibly can be, by looking for a function $\psi$ with $\operatorname{supp} \psi \subset B_{\tau}$ that minimises the MSM-residual $\left\|P_{\varrho}(I+W) P_{\tau} \psi-P_{\varrho} \phi^{z}\right\|$. The truncated approximate equation (7.4) that we have to solve can be written as

$$
\left(P_{\tau} \psi\right)(\boldsymbol{x})+\int_{[-\tau, \tau]^{2}} k(\boldsymbol{x}, \boldsymbol{y}) J(\boldsymbol{y})\left(P_{\tau} \psi\right)(\boldsymbol{y}) d \boldsymbol{y} \approx \phi^{z}(\boldsymbol{x}), \quad \boldsymbol{x} \in[-\varrho, \varrho]^{2} .
$$

As before in the case of Nyström methods, we transform this approximative equation by numerical quadrature and projection into an approximate matrix equation

$$
A \psi \approx \phi^{z}
$$

To find a solution to this equation we suggest to use an iterative solver and minimise the functional

$$
\mu(\boldsymbol{\psi}):=\left\|\boldsymbol{A} \boldsymbol{\psi}-\boldsymbol{\phi}^{z}\right\|_{2} .
$$

To be precise, we assume that the parameters $\varrho$ and $\tau$ satisfy the relations

$$
\varrho=h \cdot n \quad \text { and } \quad \tau=h \cdot m
$$

for some step-size $h>0$ and natural numbers $n, m \in \mathbb{N}$. We write the integral operator in equation (7.10) in the equivalent form as an integral over the square $[-\varrho, \varrho]^{2}$. Introducing $N:=(n, n)$ and $M:=(m, m)$, we replace $(7.10)$ by

$$
\left(P_{\tau} \psi\right)(\boldsymbol{x})+\sum_{\boldsymbol{j}=-N}^{N-1} k_{h, \boldsymbol{j}}(\boldsymbol{x}) J(h \boldsymbol{j})\left(P_{\tau} \psi\right)(h \boldsymbol{j}) \approx \phi^{z}(\boldsymbol{x}), \quad \boldsymbol{x} \in[-\varrho, \varrho]^{2},
$$

where the functions $k_{h, j}$ are given through (6.7). Restricting $\boldsymbol{x}$ to the points

$$
\left\{h \boldsymbol{i} \in \mathbb{R}^{2}:-N \leq \boldsymbol{i} \leq N-1\right\}
$$

yields

$$
\left(P_{\tau} \psi\right)(h \boldsymbol{i})+\sum_{\boldsymbol{j}=-N}^{N-1} k_{h, \boldsymbol{j}}(h \boldsymbol{i}) J(h \boldsymbol{j})\left(P_{\tau} \psi\right)(h \boldsymbol{j}) \approx \phi^{z}(h \boldsymbol{i}), \quad-N \leq \boldsymbol{i} \leq N-1 .
$$


We can write this in the form $(7.11)$, where $\boldsymbol{I}, \boldsymbol{A}, \boldsymbol{W}, \boldsymbol{P} \in \mathbb{C}^{[\times(2 N)] \times[\times(2 N)]}$ are twodimensional matrices with

$$
\begin{aligned}
(\boldsymbol{I})_{\boldsymbol{i}, \boldsymbol{j}} & :=\delta_{\boldsymbol{i}, \boldsymbol{j}}, \\
(\boldsymbol{W})_{\boldsymbol{i}, \boldsymbol{j}} & :=k_{h, \boldsymbol{j}}(h \boldsymbol{i}), \\
\left(\boldsymbol{P}_{\tau}\right)_{\boldsymbol{i}, \boldsymbol{j}} & := \begin{cases}\delta_{\boldsymbol{i}, \boldsymbol{j}}, & -M \leq \boldsymbol{i}, \boldsymbol{j} \leq M-1, \\
0, & \text { otherwise }\end{cases}
\end{aligned}
$$

for $-N \leq \boldsymbol{i}, \boldsymbol{j} \leq N-1$ and

$$
\boldsymbol{A}:=(\boldsymbol{I}+\boldsymbol{W}) \cdot \boldsymbol{P}_{\tau}
$$

and $\boldsymbol{\psi}, \boldsymbol{\phi}^{z} \in \mathbb{C}^{\times(2 N)}$ are two-dimensional arrays with

$$
\begin{aligned}
\boldsymbol{\psi}_{\boldsymbol{j}} & :=\psi(h \boldsymbol{j}), \\
\boldsymbol{\phi}_{\boldsymbol{j}}^{z} & :=\phi^{z}(h \boldsymbol{j}),
\end{aligned}
$$

for $-N \leq \boldsymbol{j} \leq N-1$.

\subsubsection{Linear least squares problems}

The problem to minimise the functional (7.12) is known as linear least squares problem. The main result for linear least squares problems is summarised in the following theorem.

Theorem 7.9. Let $A \in \mathbb{C}^{m \times n}$ and $b \in \mathbb{C}^{m}$ with $m, n \in \mathbb{N}$ and $m>n$. Then $x \in \mathbb{C}^{n}$ is a solution of the linear least squares problem

$$
\min _{x \in \mathbb{R}^{n}}\|A x-b\|_{2}
$$

if and only if $x$ is a solution of the normal equation

$$
A^{*} A x=A^{*} b .
$$

Proof. See e.g. [7, Theorem 1.1.2].

The matrix $A^{*} A$ is hermitian and positive semi-definite. Furthermore $A^{*} A$ is positive definite if and only if $N(A)=\{0\}$. In this case the normal equation possesses a unique solution $\hat{x}$ given through

$$
\hat{x}=\left(A^{*} A\right)^{-1} A^{*} b .
$$

In the case that $N(A) \neq\{0\}$, which means that $A$ does not have full rank, the normal equation and hence the linear least square problem possesses more than one 
ALGORITHM 7.1 The conjugate gradient method for linear least squares problems

\section{Input data:}

$\begin{array}{lll}A & \in \mathbb{C}^{m \times n} & \% \text { the system matrix } \\ x^{(0)} & \in \mathbb{C}^{n} & \% \text { initial guess } \\ b & \in \mathbb{C}^{m} & \% \text { the right hand side }\end{array}$

\section{Initialisation:}

$$
\begin{aligned}
& r^{(0)}=b-A x^{(0)} \\
& s^{(0)}=A^{*} r^{(0)} \\
& p^{(0)}=s^{(0)} \\
& \text { FOR } k=0,1, \ldots \text { DO } \\
& q^{(k)} \quad=A p^{(k)} \\
& \alpha_{k} \quad=\left\|s^{(k)}\right\|_{2}^{2} /\left\|q^{(k)}\right\|_{2}^{2} \\
& x^{(k+1)}=x^{(k)}+\alpha_{k} p^{(k)} \\
& r^{(k+1)}=r^{(k)}-\alpha_{k} q^{(k)} \\
& s^{(k+1)}=A^{*} r^{(k+1)} \\
& \beta_{k} \quad=\left\|s^{(k+1)}\right\|_{2}^{2} /\left\|s^{(k)}\right\|_{2}^{2} \\
& p^{(k+1)}=s^{(k+1)}+\beta_{k} p^{(k)}
\end{aligned}
$$

\section{Output:}

$x^{(k)} \quad \%$ approximation to the linear least squares problem

$s^{(k)} \quad \%$ the residual of the normal equation

solution. But under all of these solutions, there is only one with minimal norm. This solution is given through $A^{\dagger} b$, where $A^{\dagger}$ denotes the pseudoinverse or Moore-Penrose inverse of $A$.

The conjugate gradient method for least squares problem (CGLS method), as it is described e.g. in [7, Chapter 7.4], is a well suited algorithm to compute this minimal norm least squares solution. The general algorithm is shown in Algorithm 7.1. We make the following definition.

Definition 7.10. For a given matrix $B \in \mathbb{C}^{n \times n}$ and vector $c \in \mathbb{C}^{n}$ the Krylov subspace $\mathcal{K}_{k}(B, c)$ is given through

$$
\mathcal{K}_{k}(B, c):=\operatorname{span}\left\{c, B c, \ldots, B^{k-1} c\right\}
$$

Now we can state the next theorem that contains the main property of the CGLS method.

THEOREM 7.11. The $k$-th iterate $x^{(k)}$ of the CGLS method is an element of the 
affine subspace

$$
x^{(k)} \in x^{(0)}+\mathcal{K}_{k}\left(A^{*} A, s^{(0)}\right) .
$$

Furthermore, $x^{(k)}$ minimises the functional (7.12) over all elements in the affine subspace.

Proof. See e.g. [7, Chapter 7.4].

On can show that the CGLS method with initial guess $x^{(0)}$ converges to the unique solution $\tilde{x}$ of the linear least squares problem that minimises $\left\|\tilde{x}-x^{(0)}\right\|$. Thus, for the choice $x^{(0)}=0$ the CGLS method converges to the minimal norm solution $\hat{x}=A^{\dagger} b$. The convergence does not require the matrix $A^{*} A$ to be definit.

We note that the algorithm can be carried out without storing the matrices $\boldsymbol{A}$ and $\boldsymbol{A}^{*}$, if one supplies subroutines that carry out the matrix-vector product $\boldsymbol{A}^{*} \cdot \boldsymbol{\psi}$ and $\boldsymbol{A} \cdot \boldsymbol{\psi}$. Using the two-dimensional matrices $\boldsymbol{G}, \boldsymbol{L} \in \mathbb{C}^{[\times(2 N)] \times[\times(2 N)]}$ given through (6.29) and (6.30), we can write the matrices $\boldsymbol{A}$ and $\boldsymbol{A}^{*}$ as

$$
\boldsymbol{A}=(\boldsymbol{I}+\boldsymbol{L}+\boldsymbol{G}) \cdot \boldsymbol{P}_{\tau} \quad \text { and } \quad \boldsymbol{A}^{*}=\boldsymbol{P}_{\tau} \cdot\left(\boldsymbol{I}+\boldsymbol{L}^{*}+\boldsymbol{G}^{*}\right) .
$$

The matrix $\boldsymbol{L}$ and $\boldsymbol{L}^{*}$ are sparse banded block matrices with band blocks of equal band-width. Thus the matrix-vector multiplication can be done in $O(\sharp(2 N))$ operations. As in the case of the BMIA, we need to speed up the matrix-vector multiplication of the dense matrices $\boldsymbol{G}$ and $\boldsymbol{G}^{*}$.

An optimised version of the CGLS method applied to the functional (7.12) is given in pseudo code in Algorithm 7.2

For some preliminary numerical results for the case of the rough surface scattering problem we refer the reader to [31]. 
$\overline{\text { ALGORITHM 7.2 The CGLS method applied to the rough surface scattering problem }}$

\section{Input data:}
$\phi^{z} \in \mathbb{C}^{\times(2 N)}$
$\boldsymbol{\psi}_{0} \in \mathbb{C}^{\times(2 N)}$
$\%$ right hand side
$\boldsymbol{A} \in \mathbb{C}^{[\times(2 N)] \times[\times(2 N)]}$
$\%$ initial guess
Tol
$\%$ large dense rectangular matrix
$\%$ tolerance

\section{Initialisation:}

$$
\begin{aligned}
& \boldsymbol{r}=\phi^{z}-\boldsymbol{A} \cdot \boldsymbol{\psi}_{0} \\
& s \quad=A^{*} \cdot r \\
& p=s \\
& \gamma_{\text {old }}=\|\boldsymbol{s}\|_{2}^{2} \\
& \gamma_{\text {new }}=\gamma_{\text {old }} \\
& \text { WHILE } \gamma_{\text {new }}>\text { Tol DO } \\
& \boldsymbol{s} \quad \boldsymbol{A} \cdot \boldsymbol{p} \\
& \alpha \quad=\gamma_{\text {old }} /\|\boldsymbol{s}\|_{2}^{2} \\
& \boldsymbol{\psi}=\boldsymbol{\psi}+\alpha \boldsymbol{p} \\
& \boldsymbol{r}=\boldsymbol{r}-\alpha \boldsymbol{s} \\
& \boldsymbol{s}=\boldsymbol{A}^{*} \cdot \boldsymbol{r} \\
& \gamma_{\text {new }}=\|\boldsymbol{s}\|_{2}^{2} \\
& \beta=\gamma_{\text {new }} / \gamma_{\text {old }} \\
& \boldsymbol{p}=\boldsymbol{s}+\beta \boldsymbol{p} \\
& \gamma_{\text {old }}=\gamma_{\text {new }}
\end{aligned}
$$

END

\section{Output:}

$\psi$. 
Chapter 7 The Multi-Section Method 


\section{Chapter 8}

\section{Fast matrix-vector multiplications for integral operators with difference kernels}

In this chapter we present the use of the fast Fourier transform (FFT) to speed up the matrix-vector multiplication for the fully discrete version of certain integral operators. More precisely, we are interested in the multi-dimensional analogon of integral operators of the form

$$
(B \psi)(x):=\int_{a}^{b} k(x-y) \psi(y) d y, \quad x \in[a, b],
$$

for real numbers $a<b$ in the cases, where

(i) $k$ and $\psi$ are piecewise, continuous periodic functions with period $b-a$,

(ii) $k$ and $\psi$ are continuous functions on $[-(b-a), b-a]$ respectively $[a, b]$.

A simple computation shows that

$$
\begin{aligned}
(B \psi)(x+a) & =\int_{a}^{b} k(x-(y-a)) \psi(y) d y \\
& =\int_{0}^{b-a} k(x-z) \psi(z+a) d z, \quad x \in(0, b-a) .
\end{aligned}
$$

Thus we see that it suffices to consider the above two cases only for operators of the form

$$
(A \psi)(x):=\int_{0}^{p} k(x-y) \psi(y) d y, \quad x \in[0, p]
$$

for some $p:=b-a>0$.

In analogy to the discretisation (5.2) we introduce a semi-discrete approximation in the form

$$
\left(A_{h} \psi\right)(x):=\sum_{j=0}^{N-1} k_{h, j}(x) \psi(h j), \quad x \in[0, p],
$$


where

$$
k_{h, j}(x):=h k(x-h j), \quad j \in \mathbb{Z}, x \in[0, p]
$$

and $h:=p / N$ denotes the step-size for $N \in \mathbb{N}$.

The fully discrete version

$$
\left(A_{h} \psi\right)(i h)=\sum_{j=0}^{N-1} k_{h, j}(i h) \psi(h j), \quad i=0, \ldots, N-1
$$

has a matrix representation $\boldsymbol{A}$ with

$$
(\boldsymbol{A})_{i, j}=h k(h(i-j)), \quad i, j=0, \ldots, N-1 .
$$

Hence the $i$-th entry of the matrix-vector product of the matrix $\boldsymbol{A}$ with a vector $\boldsymbol{\psi}=\left(\boldsymbol{\psi}_{i}\right)_{i=0, \ldots, N-1} \in \mathbb{C}^{N \times 1}$ with

$$
\boldsymbol{\psi}_{j}:=\psi(h j), \quad j \in \mathbb{Z},
$$

is given through

$$
(\boldsymbol{A} \boldsymbol{\psi})_{i}=h \sum_{j=0}^{N-1} k(h(i-j)) \boldsymbol{\psi}_{j}, \quad k=0, \ldots, N-1 .
$$

If done in a naive way, this matrix-vector multiplication needs $O\left(N^{2}\right)$ evaluations of multiplications and summations. Fortunately, the matrix has a very special structure that can be exploited. Due to the equidistantly spaced grid we see that in the case of a periodic kernel function, the matrix $\boldsymbol{A}$ is of the form

$$
\left(\begin{array}{ccccc}
c_{0} & c_{1} & \ldots & c_{N-2} & c_{N-1} \\
c_{N-1} & c_{0} & c_{1} & \ldots & c_{N-2} \\
\vdots & \ddots & \ddots & \ddots & \vdots \\
c_{2} & \ldots & c_{N-1} & c_{0} & c_{1} \\
c_{1} & c_{2} & \ldots & c_{N-1} & c_{0}
\end{array}\right)
$$

where $c_{j}:=h k(h j)$ for $j \in \mathbb{Z}$. Such a matrix is called circulant matrix. A circulant matrix is obviously fully determined by its first row or column.

In the case of a non-periodic kernel function, the matrix $\boldsymbol{A}$ is of the form

$$
\left(\begin{array}{ccccc}
t_{0} & t_{1} & \ldots & t_{N-2} & t_{N-1} \\
t_{-1} & t_{0} & t_{1} & \ldots & t_{N-2} \\
\vdots & \ddots & \ddots & \ddots & \vdots \\
t_{-N+2} & \ldots & t_{-1} & t_{0} & t_{1} \\
t_{-N+1} & t_{-N+2} & \ldots & t_{-1} & t_{0}
\end{array}\right)
$$


where $t_{j}:=h k(h j)$ for $j \in \mathbb{Z}$. Such a matrix is called Toeplitz matrix and it is fully determined by its first row and column.

It is possible to design fast matrix-vector multiplications for these kind of matrices that reduce the normal amount of work from $O\left(N^{2}\right)$ down to $O(N \log N)$. These fast algorithms are based on the following two properties of circulant and Toeplitz matrices:

- any circulant matrix is diagonalised by the Fourier matrix, i.e. the eigenvectors of a circulant matrix are the columns of the Fourier matrix,

- any Toeplitz matrix of size $N \times N$ can be embedded into a circulant matrix of size $2 N \times 2 N$, cf. EXAMPle 8.3.

We show in the next section that the discretisation of integral operators with difference kernels in $\mathbb{R}^{d}$ lead to special matrices that are generalisation of circulant and Toeplitz matrices. They are $d$-level block matrices, where each block and level is circular or toeplitz. Though we only need two- and three-level block matrices we give a general approach that works for arbitrary dimensions.

\subsection{The multi-dimensional case for periodic kernel}

Consider, for some $p \in \mathbb{R}^{d}$ with $p>0$, the multi-dimensional analogon of (8.1), i.e.

$$
(A \psi)(x):=\int_{[0, p]} k(x-y) \psi(y) d y, \quad x \in[0, p]
$$

with multi-periodic kernel and density function with period vector $p$. For $N \in \mathbb{N}^{d}$ we define the step size

$$
h:=p \div 2 N \in \mathbb{R}_{>0}^{d} .
$$

Note that the step-size is a vector so that the grid points are given through $h \odot j$ for $j \in \mathbb{Z}^{d}$.

REMARK 8.1. We chose an even number of discretisation points, as this will enable us later on to use the fast Fourier transform for the matrix-vector multiplication. This will work best if $N$ is some power of two.

The semi-discrete and fully discrete approximation are given through

$$
\left(A_{h} \psi\right)(x):=\sum_{j=0}^{2 N-1} k_{h, j}(x) \psi(h \odot j), \quad x \in[0, p]
$$

with

$$
k_{h, j}(x):=\sharp(h) k(x-h \odot j), \quad j \in \mathbb{Z}^{d}, x \in[0, p]
$$


and

$$
\left(A_{h} \psi\right)(h \odot i):=\sum_{j=0}^{2 N-1} k_{h, j}(h \odot i) \psi(h \odot j), \quad i=0, \ldots, 2 N-1,
$$

respectively. The matrix representation of the fully discrete version is a $d$-level block circulant matrix $\boldsymbol{A} \in \mathbb{C}^{[\times(2 N)] \times[\times(2 N)]}$ with

$$
(\boldsymbol{A})_{i, j}=\sharp(h) k(h \odot(i-j)), \quad 0 \leq i, j \leq 2 N-1 .
$$

A different way to get the same discretisation is the following. Instead of replacing the integral by a quadrature formula (we are using the composite trapezoidal rule), we replace the kernel and density by their $d$-dimensional trigonometric interpolation polynomial of degree at most $|N|$. Thus the semi-discrete approximation for the Fourier method is given through

$$
\left(A_{h}^{\mathrm{DFT}} \psi\right)(x):=\int_{[0, p]} k_{N}(x-y) \psi_{N}(y) d y, \quad x \in[0, p],
$$

where $k_{N}$ and $\psi_{N}$ are the $d$-dimensional trigonometric interpolation polynomials given through (4.6) with coefficients given through (4.7). Inserting the corresponding representations of $k_{N}$ and $\psi_{N}$, we can use the convolution theorem for periodic functions (THEOREM 4.3) to rewrite the integral to yield

$$
\begin{aligned}
\left(A_{h}^{\mathrm{DFT}} \psi\right)(x) & =\int_{[0, p]}\left(\sum_{m=-N}^{N-1} \tilde{k}(m) e^{2 \pi i\langle m,(x-y) \div p\rangle}\right)\left(\sum_{n=-N}^{N-1} \tilde{\psi}(n) e^{\pi i\langle n, y \div p\rangle}\right) d y \\
& =\sharp(p) \sum_{m=-N}^{N-1} \tilde{k}(m) \tilde{\psi}(m) e^{2 \pi i\langle m, x \div p\rangle}, \quad x \in[0, p] .
\end{aligned}
$$

The fully discrete version is given through

$$
\left(A_{h}^{\mathrm{DFT}} \psi\right)(h \odot l)=\sharp(p) \sum_{m=-N}^{N-1} \tilde{k}(m) \tilde{\psi}(m) e^{2 \pi i\langle m, l \div 2 N\rangle}, \quad l=0, \ldots, 2 N-1 .
$$

We note that this is a discrete version of the convolution theorem for periodic functions (THEOREM 4.3) that can be used for fast summation algorithm. To compute this expression we see that it suffices to calculate three $d$-dimensional discrete Fourier transform (DFT) of length $N^{*}:=\sharp(2 N)$. If this is done with a $d$-dimensional FFT, we only need $O\left(3 \cdot N^{*} \log \left(N^{*}\right)\right)$ operations as compared to $O\left(N^{* 2}\right)$ operations for the standard algorithm.

The following Lemma shows that both algorithm give exactly the same result.

LEMma 8.2. $\left(A_{h}^{D F T} \psi\right)(h \odot l)=\left(A_{h} \psi\right)(h \odot l), \quad l=0, \ldots, 2 N-1$. 
Proof. We calculate that

$$
\begin{aligned}
\left(A_{h}^{\mathrm{DFT}} \psi\right)(h \odot l) & =\sharp(p) \sum_{m=-N}^{N-1} \tilde{k}(m) \tilde{\psi}(m) e^{2 \pi i\langle m, l \div 2 N\rangle} \\
& \left.=\sharp(p) \sum_{m=-N}^{N-1}\left[\frac{1}{\sharp(2 N)} \sum_{j^{\prime}=0}^{2 N-1} k\left(h \odot j^{\prime}\right) e^{-2 \pi i\left\langle m, j^{\prime} \div 2 N\right\rangle}\right]\right] \\
& {\left[\frac{1}{\sharp(2 N)} \sum_{j=0}^{2 N-1} \psi(h \odot j) e^{-2 \pi i\langle m, j \div 2 N\rangle}\right] e^{2 \pi i\langle m, l \div 2 N\rangle} } \\
& =\frac{\sharp(p)}{[\sharp(2 N)]^{2}} \sum_{j^{\prime}=0}^{2 N-1} \sum_{j=0}^{2 N-1} k\left(h \odot j^{\prime}\right) \psi(h \odot j) \sum_{m=-N}^{N-1} e^{-2 \pi i\left\langle m,\left(j+j^{\prime}-l\right) \div 2 N\right\rangle} \\
& =\frac{\sharp(h)}{\sharp(2 N)} \sum_{j^{\prime}=0}^{2 N-1} \sum_{j=0}^{2 N-1} k\left(h \odot j^{\prime}\right) \psi(h \odot j) \sharp(2 N) \delta_{j^{\prime}, l-j} \\
& =\sharp(h) \sum_{j=0}^{2 N-1} k(h \odot(l-j)) \psi(h \odot j) \\
& =\left(A_{h} \psi\right)(h \odot l), \quad l=0, \ldots, 2 N-1 .
\end{aligned}
$$

\subsection{The multi-dimensional case for non-periodic kernel}

Now we consider the case of a non-periodic kernel function and density. For an integral operator of the form

$$
(A \psi)(x):=\int_{[0, p]} k(x-y) \psi(y) d y, \quad x \in[0, p]
$$

the kernel function must be known on the cube $[-p, p]$, whereas the density is only defined in $[0, p]$. A straightforward discretisation of this problem would yield a $d$ level Toeplitz matrix. To get a fast version for the matrix-vector multiplication we reduce this problem to the case of a periodic kernel and density. Denote by $k_{\text {per }}$ the multi-periodic extension of $k$ with period vector $2 p$. For $\psi \in C([0, p])$ we define

$$
\psi_{0}(x):= \begin{cases}\psi(x), & x \in[0, p) \\ 0, & x \in[0,2 p] \backslash[0, p)\end{cases}
$$


and denote the multi-periodic extension of $\psi_{0}$ with period vector $2 p$ by $\psi_{0, \text { per }}$. Then we have the representation

$$
\int_{[0, p]} k(x-y) \psi(y) d y=\int_{[0,2 p]} k_{\text {per }}(x-y) \psi_{0, \text { per }}(y) d y, \quad x \in[0, p] .
$$

i.e. on the first half of the interval $[0,2 p]$ the righthand side coincides with the original operator. Now we can use the same discretisation as in the case of piecewise continuous periodic functions that we described in the previous section.

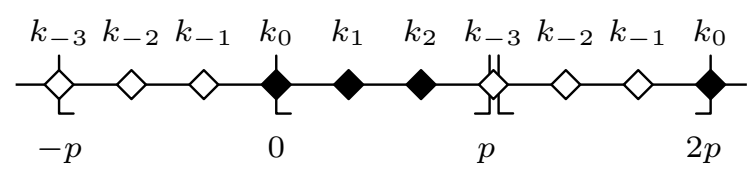

FIGURE 8.1:

EXAMPLE 8.3. We illustrate this for the case $N=3$. The fully discrete version of the integral operator is given through

$$
\left(A_{h} \psi\right)(h i)=\sum_{j=0}^{2} h k(h(i-j)) \psi(h j), \quad i=0, \ldots, 2 .
$$

Using the convention $k_{l}:=h k(h l), l \in \mathbb{Z}$ and $\psi_{j}:=\psi(h l)$ we can write the previous formula as the matrix-vector product

$$
\left(\begin{array}{ccc}
k_{0} & k_{1} & k_{2} \\
k_{-1} & k_{0} & k_{1} \\
k_{-2} & k_{-1} & k_{0}
\end{array}\right)\left(\begin{array}{c}
\psi_{0} \\
\psi_{1} \\
\psi_{2}
\end{array}\right)
$$

The fully discrete version of the periodised integral operator is given through

$$
\sum_{j=0}^{5} h k_{p e r}(h(i-j)) \psi_{0, p e r}(h j), \quad i=0, \ldots, 5 .
$$

As before this can be written as matrix-vector product

$$
\left(\begin{array}{ccc|ccc}
k_{0} & k_{1} & k_{2} & \boldsymbol{k}_{-\mathbf{3}} & k_{-2} & k_{-1} \\
k_{-1} & k_{0} & k_{1} & k_{2} & \boldsymbol{k}_{-\mathbf{3}} & k_{-2} \\
k_{-2} & k_{-1} & k_{0} & k_{1} & k_{2} & \boldsymbol{k}_{-\mathbf{3}} \\
\hline \boldsymbol{k}_{-\mathbf{3}} & k_{-2} & k_{-1} & k_{0} & k_{1} & k_{2} \\
k_{2} & \boldsymbol{k}_{-\mathbf{3}} & k_{-2} & k_{-1} & k_{0} & k_{1} \\
k_{1} & k_{2} & \boldsymbol{k}_{-\mathbf{3}} & k_{-2} & k_{-1} & k_{0}
\end{array}\right)\left(\begin{array}{c}
\psi_{0} \\
\psi_{1} \\
\psi_{2} \\
0 \\
0 \\
0
\end{array}\right),
$$


where we used the periodicity condition of the kernel function and the zero extension of the density. Note that the entry $\boldsymbol{k}_{-\mathbf{3}}$ can be chosen arbitrarily. For reasons of simplicity we use the value at the left endpoint of the interval [-p, $p]$, cf. FIGURE 8.1. This example explains the embedding of an $N \times N$ Toeplitz matrix into a $2 N \times 2 N$ circulant matrix, as was mentioned at the beginning of this chapter.

If we choose a discretisation for $N \in \mathbb{N}^{d}$ with a total of $N^{*}:=\sharp(N)$ unknowns, we can reduce the necessary floating point operations (addition, multiplication, etc.) from $N^{* 2}$ to $3 \cdot 2^{d} N^{*} \log _{2}\left(2^{d} N^{*}\right)$.

We illustrate the simplicity of this algorithm in the following two-dimensional example.

EXAMPle 8.4. Assume we are given the kernel function $k:\left(x_{1}, x_{2}\right) \mapsto \sin \left(\left(x_{1}+\right.\right.$ 1) $\left.\left(x_{1}-x_{2}\right)\right)$ and density $\psi:\left(x_{1}, x_{2}\right) \mapsto x_{1}^{3}-x_{2}$ and want to calculate the fully discrete version of $(8.6)$ in the case $p=(2,3)$ for a grid with $N=(18,20)$. Then we can realise this in the following few lines of MATLAB code, cf. LisTing 8.1.

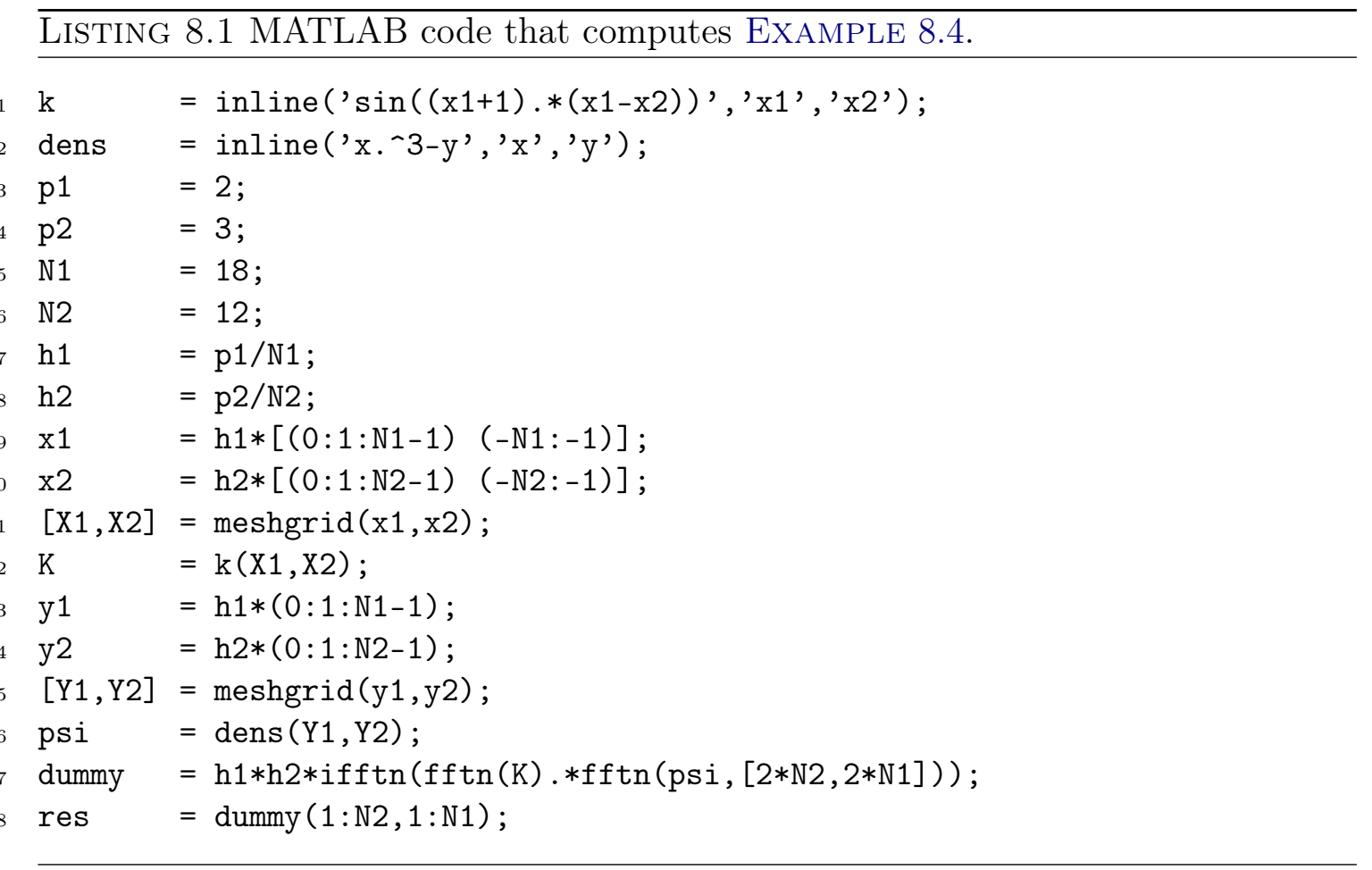

The periodisation of the kernel function is done in the lines 9-11, by defining an appropriate grid. Line 17 is a direct implementation of (8.5). The zero padding of the density, cf. (8.7), is done implicitly by MATLAB's fftn routine through the command $\mathrm{fftn}(\mathrm{psi},[2 * \mathrm{~N} 2,2 * \mathrm{~N} 1]))$. The solution that was calculated on the grid inside $[0,2 p]$ is restricted to the grid points of interest inside $[0, p]$ in line 18. 


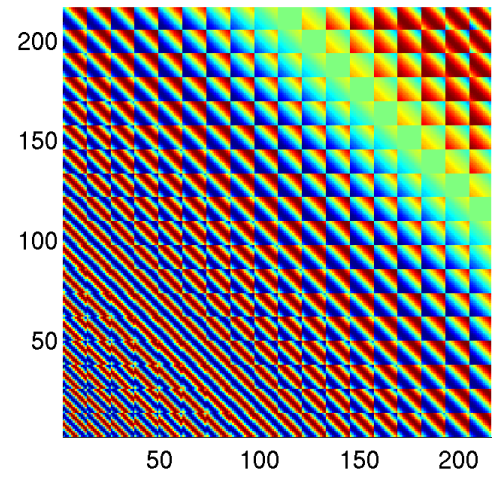

(a) The 2-level block Toeplitz matrix that represents the integral operator of EXAMPLE 8.4

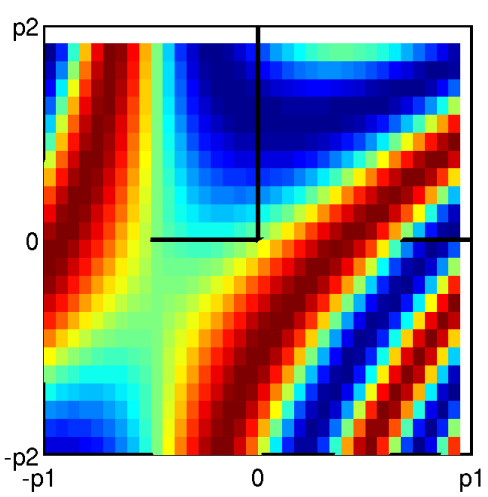

(c) The kernel function of Example 8.4 on $[-p, p]$

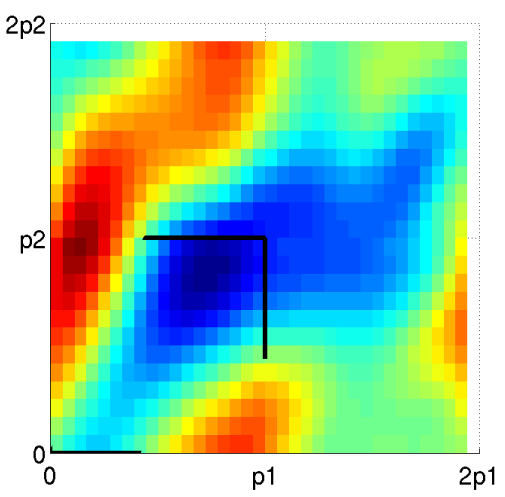

(b) The matrix-vector product of the discrete version of the operator $A$ with the density $\psi$ is in the lower left quadrant

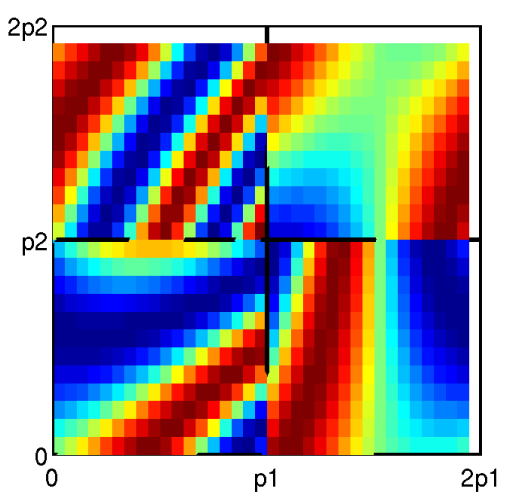

(d) The periodised kernel function of ExamPLE 8.4 on $[0,2 p]$

FiguRE 8.2: Illustration of EXAMPLE 8.4 


\section{Chapter 9}

\section{A new view on canonical grid methods}

This chapter introduces the canonical grid method that allows to accelerate the matrix-vector multiplication of the fully discrete version for a certain class of integral operators. The integral operators that occur in the case of rough scattering problems are prominent example of this class. The idea of this method is to replace the integral kernel by some appropriate approximation that allows one to handle the corresponding operator approximation with FFT methods. There are a lot of different ways to derive such an approximation. The original idea is based on Taylor expansion and we give a short review in Section 9.1. We continue our presentation with another version that is based on interpolation rather than Taylor expansions. Our introduction to the canonical grid method follows in large parts the presentation given in [19]. Having reviewed these two variants we give a novel derivation that in some sense generalises the interpolation method.

We like to point out that the error analysis in Section 9.2.1 is based on considerations in [5].

\subsection{Canonical grid method revisited}

We consider the following prototype of an integral operator

$$
(W \psi)(\boldsymbol{x}):=\int_{R} K(\boldsymbol{x}, \boldsymbol{y}) J(\boldsymbol{y}) \psi(\boldsymbol{y}) d \boldsymbol{y}, \quad \boldsymbol{x} \in R,
$$

where $R=[-\varrho, \varrho]^{2} \subset \mathbb{R}^{2}, J(\boldsymbol{y})=\sqrt{1+|\nabla f(\boldsymbol{y})|^{2}}$ denotes the surface area element and the kernel allows a representation in the form

$$
K(\boldsymbol{x}, \boldsymbol{y})=k(\boldsymbol{x}-\boldsymbol{y}, f(\boldsymbol{x})-f(\boldsymbol{y})),
$$

where $k: \mathbb{R}^{2} \times \mathbb{R} \rightarrow \mathbb{C}$ satisfies the following two conditions:

(C1) $k\left(\cdot, x_{3}\right)$ is a $n$-times continuously differentiable function on $\mathbb{R}^{2}$ for some $n \geq 0$. 
(C2) $k(\boldsymbol{x}, \cdot)$ is an analytical function for each $\boldsymbol{x} \in \mathbb{R}^{2}$.

The idea of the canonical grid method is to approximate the kernel function $K(\cdot, \cdot)$ by some function $K_{M}(\cdot, \cdot)$, which takes the form

$$
K_{M}(\boldsymbol{x}, \boldsymbol{y})=\sum_{j=1}^{C_{M}} a_{j, M}(\boldsymbol{x}) K_{j, M}(\boldsymbol{x}-\boldsymbol{y}) b_{j, M}(\boldsymbol{y}),
$$

where $a_{j, M}, K_{j, M}$ and $b_{j, M}$ are functions to be determined. $M$ is an integer parameter controlling the accuracy of the approximation, such that $K_{M}(\boldsymbol{x}, \boldsymbol{y}) \rightarrow K(\boldsymbol{x}, \boldsymbol{y})$ as $M \rightarrow \infty$. The integral operator

$$
\left(W_{M} \psi\right)(\boldsymbol{x}):=\int_{R} K_{M}(\boldsymbol{x}, \boldsymbol{y}) J(\boldsymbol{y}) \psi(\boldsymbol{y}) d \boldsymbol{y}, \quad \boldsymbol{x} \in R,
$$

which we can write as the following sum and products

$$
\left(W_{M} \psi\right)(\boldsymbol{x})=\sum_{j=1}^{C_{M}} a_{j, M}(\boldsymbol{x}) \int_{R} K_{j, M}(\boldsymbol{x}-\boldsymbol{y}) b_{j, M}(\boldsymbol{y}) J(\boldsymbol{y}) \psi(\boldsymbol{y}) d \boldsymbol{y}, \quad \boldsymbol{x} \in R,
$$

is an approximation to $W$ which is suitable for the fast methods from Section 8.2. In fact, the fully discrete form of this operator has a matrix representation

$$
\boldsymbol{A}^{(M)}=\sum_{j=1}^{C_{M}} \boldsymbol{A}^{j, M} \boldsymbol{T}^{j, M} \boldsymbol{B}^{j, M}
$$

where $\boldsymbol{A}^{j, M}$ and $\boldsymbol{B}^{j, M}$ are diagonal matrices and $\boldsymbol{T}^{j, M}$ are 2-level block Toeplitz matrices.

\subsubsection{Deriving a kernel decomposition}

The original canonical grid method, as introduced in [45], uses an Taylor series expansion of $k(\boldsymbol{x}-\boldsymbol{y}, f(\boldsymbol{x})-f(\boldsymbol{y}))$ with respect to $f(\boldsymbol{x})-f(\boldsymbol{y})$ around 0 to derive the approximation (9.3), i.e.

$$
\begin{aligned}
k(\boldsymbol{x}-\boldsymbol{y}, f(\boldsymbol{x})-f(\boldsymbol{y})) & \left.\approx \sum_{j=0}^{M-1} \frac{1}{j !} \frac{\partial^{j}}{\partial u^{j}} k(\boldsymbol{x}-\boldsymbol{y}, u)\right|_{u=0}[f(\boldsymbol{x})-f(\boldsymbol{y})]^{j} \\
& =\left.\sum_{j=0}^{M-1} \sum_{i=0}^{j}\left(\begin{array}{l}
j \\
i
\end{array}\right) \frac{1}{j !} \frac{\partial^{j}}{\partial u^{j}} k(\boldsymbol{x}-\boldsymbol{y}, u)\right|_{u=0} f(\boldsymbol{x})^{j-i} f(\boldsymbol{y})^{i},
\end{aligned}
$$

where

$$
c_{i j}:=\frac{(-1)^{i}}{j !}\left(\begin{array}{l}
j \\
i
\end{array}\right)=\frac{(-1)^{i}}{(j-i) ! i !}
$$


and where in the last step we have used the binomial theorem.

As it was pointed out in [19], the Taylor series is slowly convergent so that the method is only effective for rough surfaces of small elevation relative to the wavelength. An extension of this method is described in [62]. Their idea was to use different expansion points for the Taylor series in the interval of interest. Thus one can reduce the number of expansion terms while at the same time keeping the accuracy over the full range of the surface elevation.

A very different approach was introduced in [19] and [6], where the Taylor expansion was replaced by an interpolation scheme in the vertical direction. We explain this now in some more detail.

Let $f_{R}^{-}$and $f_{R}^{+}$denote the infimum and supremum of $f$ on $R$, i.e.

$$
f_{R}^{-}:=\inf _{\boldsymbol{x} \in R} f(\boldsymbol{x}) \quad \text { and } \quad f_{R}^{+}:=\sup _{\boldsymbol{x} \in R} f(\boldsymbol{x})
$$

and let $a_{3}$ and $b_{3}$ be real constants such that

$$
a_{3} \leq f_{R}^{-}<f_{R}^{+} \leq b_{3} .
$$

Then we choose $M$ distinct points $z_{j}, j=0, \ldots, M-1$ in the interval $\left[a_{3}, b_{3}\right]$ and a corresponding set of linearly independent basis function

$$
\mathcal{L}_{M}:=\left\{L_{j}: j=0, \ldots, M-1\right\}
$$

for which we assume that they form a Lagrange basis, i.e.

$$
L_{j}\left(z_{i}\right)=\delta_{j, i} \quad i, j=0, \ldots, M-1 .
$$

With the help of this basis functions we define an interpolation operator $P_{M}$ through

$$
\left(P_{M} f\right)(z):=\sum_{j=0}^{M-1} f\left(z_{j}\right) L_{j}(z), \quad z \in\left[a_{3}, b_{3}\right] .
$$

We approximate the function $k(\boldsymbol{x}-\boldsymbol{y}, u-v)$ through

$$
k(\boldsymbol{x}-\boldsymbol{y}, u-v) \approx \sum_{j=0}^{M-1} \sum_{j^{\prime}=0}^{M-1} L_{j}(u) k\left(\boldsymbol{x}-\boldsymbol{y}, z_{j}-z_{j^{\prime}}\right) L_{j^{\prime}}(v),
$$

i.e. by first applying $P_{M}$ to $k(\boldsymbol{x}-\boldsymbol{y}, \cdot-v)$ or $k(\boldsymbol{x}-\boldsymbol{y}, u-\cdot)$ separately and then combining theses approximations in a second step. Thus we get the approximation

$$
K_{M}(\boldsymbol{x}, \boldsymbol{y}):=\sum_{j=0}^{M-1} \sum_{j^{\prime}=0}^{M-1} L_{j}(f(\boldsymbol{x})) k\left(\boldsymbol{x}-\boldsymbol{y}, z_{j}-z_{j^{\prime}}\right) L_{j^{\prime}}(f(\boldsymbol{y})) .
$$

In [19] and [6] Chebyshev polynomials were used for the interpolation. In this case the interval for the approximation $\left[a_{3}, b_{3}\right]$ is given through the choice $a_{3}=f_{R}^{-}$and $b_{3}=f_{R}^{+}$. 
REMARK 9.1. From these explanations we see that the convergence rate of the canonical grid method, is only dependend on the smoothness of the function

$$
\left[-\left(b_{3}-a_{3}\right),\left(b_{3}-a_{3}\right)\right]: x_{3} \mapsto k\left(\boldsymbol{x}, x_{3}\right), \quad \boldsymbol{x} \in R
$$

and not on the smoothness of the scattering surface, i.e. the surface height function $f$.

\subsection{A novel approach}

To explain our view on the canonical grid method, we rewrite the operator (9.1) in the form

$$
(W \psi)(\boldsymbol{x})=\int_{R} k((\boldsymbol{x}, f(\boldsymbol{x}))-(\boldsymbol{y}, f(\boldsymbol{y}))) J(\boldsymbol{y}) \psi(\boldsymbol{y}) d \boldsymbol{y}, \quad \boldsymbol{x} \in R .
$$

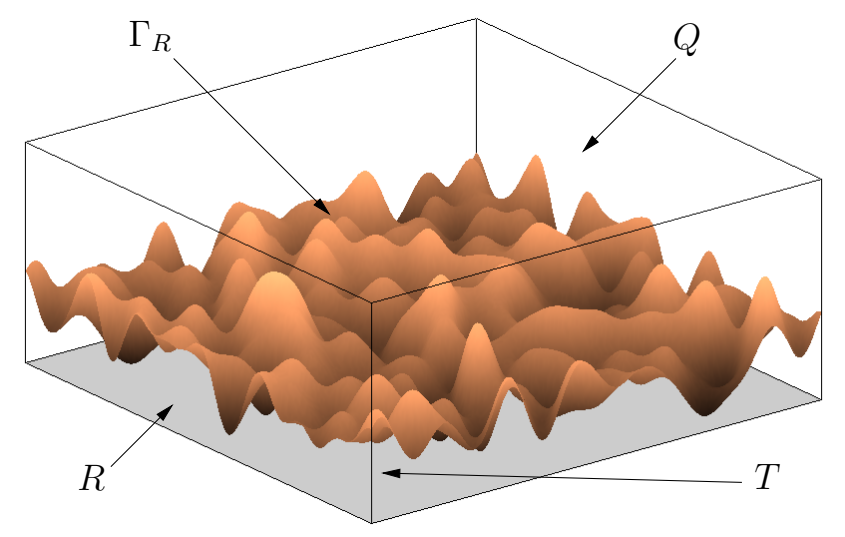

FiguRE 9.1: The cube $Q$ containing the surface patch $\Gamma_{R}$.

For such an operator we define the associated cube potential

$$
(\mathcal{W} \psi)(x):=\int_{R} k(x-(\boldsymbol{y}, f(\boldsymbol{y}))) J(\boldsymbol{y}) \psi(\boldsymbol{y}) d \boldsymbol{y}, \quad x=\left(\boldsymbol{x}, x_{3}\right) \in Q,
$$

where $Q$ denotes the cube $R \times T$ and $T$ denotes the interval $\left[a_{3}, b_{3}\right]$ for some real numbers $a_{3}, b_{3}$ that satisfy the condition (9.6), i.e.

$$
Q=[-\varrho, \varrho]^{2} \times\left[a_{3}, b_{3}\right] \subset \mathbb{R}^{3}
$$

By construction $Q$ contains the surface patch $\Gamma_{R} \subset \Gamma$ above the supporting rectangle $R$ given by

$$
\Gamma_{R}=\{(\boldsymbol{x}, f(\boldsymbol{x})): \boldsymbol{x} \in R\}
$$


An illustration is shown in FIGURE 9.1.

Depending on the smoothness condition $(\mathbf{C 1})$ it defines a function in $Q$ that is at least $n$-times continuously differentiable and furthermore we have the relation

$$
(W \psi)(\boldsymbol{x})=(\mathcal{W} \psi)(\boldsymbol{x}, f(\boldsymbol{x})), \quad \boldsymbol{x} \in R
$$

By formally inserting a Dirac delta distribution we can write the cube potential in the form

$$
\begin{aligned}
(\mathcal{W} \psi)(x) & =\int_{R} k(x-(\boldsymbol{y}, f(\boldsymbol{y}))) J(\boldsymbol{y}) \psi(\boldsymbol{y}) d \boldsymbol{y} \\
& =\int_{R} \int_{T} k(x-(\boldsymbol{y}, f(\boldsymbol{y}))) J(\boldsymbol{y}) \psi(\boldsymbol{y}) \delta\left(f(\boldsymbol{y})-y_{3}\right) d y_{3} d \boldsymbol{y} \\
& =\int_{Q} k(x-y) \Psi(y) d y, \quad x \in Q,
\end{aligned}
$$

where

$$
\Psi(y):=J(\boldsymbol{y}) \psi(\boldsymbol{y}) \delta\left(f(\boldsymbol{y})-y_{3}\right), \quad y=\left(\boldsymbol{y}, y_{3}\right) \in Q .
$$

The relation (9.9) can be written as

$$
(W \psi)(\boldsymbol{x})=\int_{T} \delta\left(f(\boldsymbol{x})-x_{3}\right)(\mathcal{W} \psi)\left(\boldsymbol{x}, x_{3}\right) d x_{3}, \quad \boldsymbol{x} \in R .
$$

After these preparations we can describe the main idea of our novel algorithm that consists basically of two steps:

Step 1 We use the representation (9.11) together with the fast methods described in Section 8.2 and calculate the cube potential on a regular grid in $Q$. This can be done very efficiently with three 3D-FFT's.

Step 2 We use the formula (9.13) to compute an approximation to the potential $W$. The integral is approximated by the composite trapezoidal rule with points that match the grid points inside $Q$. The number of operations that are necessary for the second step scales linearly with the number of unknowns in the grid in $Q$.

An implementation of this algorithm in MATLAB is presented in LISTING 10.1. To make this approach suitable for a numerical discretisation we approximate the Dirac delta distribution by a Dirac delta sequence. In general a Dirac delta sequence is a family of functions (often continuous) $\left\{\delta_{h}\right\}_{h>0}$ such that

$$
\lim _{h \rightarrow 0} \int_{\mathbb{R}} g(t) \delta_{h}(x-t) d t=g(x), \quad x \in \mathbb{R},
$$


for any $g$ in a reasonable class of test functions. Sometimes one requests also that

$$
\int_{\mathbb{R}} \delta_{h}(x-t) d t=1, \quad h>0 .
$$

This means that the constant functions are reproduced exactly for any choice of $h$. The intuitive picture that one should have in mind is a family of functions that get more and more concentrated or peaked at zero for $h \rightarrow 0$. A prominent example are the scaled Gaussians

$$
\delta_{h}(x)=\frac{1}{h \sqrt{\pi}} e^{-x^{2} / h^{2}},
$$

where the scale factor has been chosen to satisfy the condition (9.15).

\subsubsection{On a good choice of Dirac delta sequences}

We should ask the following questions:

- What is a good choice for a Dirac delta sequence?

- Can we find criteria that a good Dirac delta sequence should fulfil?

We want to use the Dirac delta sequence in a discrete setting, thus we are interested to find a family of functions, now called $d_{h}$ to avoid confusions with the sequence $\delta_{h}$, that satisfy the discrete analogon of (9.14), i.e.

$$
\lim _{h \rightarrow 0} h \sum_{j \in \mathbb{Z}} g(h j) d_{h}(x-h j)=g(x), \quad x \in \mathbb{R}
$$

for $g$ in a certain class of functions. Thus we are interested in understanding approximations of the form

$$
g(x) \approx h \sum_{j \in \mathbb{Z}} g(h j) d_{h}(x-h j), \quad x \in \mathbb{R}
$$

which is a sum of translates of one fixed kernel function $d_{h}$. If $d_{h}$ satisfies the interpolation condition

$$
g(h i)=h \sum_{j \in \mathbb{Z}} g(h j) d_{h}(h i-h j), \quad i \in \mathbb{Z},
$$

we call the function $d_{h}$ an interpolation kernel otherwise quasi-interpolation kernel.

Sometimes it is possible to make this approximation exact for a certain class of functions. In this case we can interpret (9.16) as a kind of sampling formula. The Shannon sampling theorem, also known as Whittaker-Kotel'nikov-Shannon sampling theorem, is a prominent example of an interpolatory type of the above formula. 
ThEOREM 9.2 (Whittaker-Kotel'nikov-Shannon sampling theorem). Let $g \in L^{2}(\mathbb{R})$ be a band-limited function with band-width $b$ and let

$$
h \leq \frac{\pi}{b}
$$

Then $g$ is completely determined by its values $g(h j), j \in \mathbb{Z}$ on a regular grid and

$$
g(x)=\sum_{j \in \mathbb{Z}} g(h j) \operatorname{sinc}\left(\pi\left(\frac{x}{h}-j\right)\right), \quad x \in \mathbb{R} .
$$

REMARK 9.3. It follows from

$$
\sum_{j \in \mathbb{Z}} g(h j) \operatorname{sinc}(\pi(i-j))=\sum_{j \in \mathbb{Z}} g(h j) \delta_{i j}=g(h i)
$$

where $\delta_{i j}$ denotes the Kronecker delta, that the right hand side of (9.17) defines an interpolation operator. Furthermore one can show, see e.g. [33], that the constant function $g(x)=1$ for $x \in \mathbb{R}$ is reproduced, i.e.

$$
1=\sum_{j \in \mathbb{Z}} \operatorname{sinc}\left(\pi\left(\frac{x}{h}-j\right)\right), \quad x \in \mathbb{R} .
$$

Thus we see that the choice $d_{h}=d_{h}^{\text {Shan }}$ with

$$
d_{h}^{\text {Shan }}(x):=\frac{1}{h} \operatorname{sinc}\left(\pi \frac{x}{h}\right), \quad x \in \mathbb{R}, \quad 0<h<\frac{\pi}{b},
$$

fits our general approach. One drawback of this choice however is that the support of $d_{h}^{\text {Shan }}$ is unbounded, which means that we have to sum up an infinite series. For the application we have in mind we are interested to approximate the function $g$ only in a small interval. Hence we assume that the functions $d_{h}$ have a local support of a few step sizes, say

$$
d_{h}(x)=0, \quad|x|>L h,
$$

for some $L \in \mathbb{N}$ and that (9.16) is exact for constants, i.e.

$$
h \sum_{j \in \mathbb{Z}} d_{h}(x-h j)=1, \quad x \in \mathbb{R} .
$$

In addition we request that there exists a constant $c>0$ such that

$$
h \sum_{j \in \mathbb{Z}}\left|d_{h}(x-h j)\right| \leq c, \quad x \in \mathbb{R} .
$$

This rather mild condition is surely fulfilled, if e.g. the function $d_{h}$ is continuous or piecewise continuous on $[-L h, L h]$. For a given $x \in \mathbb{R}$ there exists a uniquely determined index $j^{*} \in \mathbb{Z}$ so that

$$
h j^{*} \leq x<h\left(j^{*}+1\right) .
$$


The finite support condition (9.19) ensures that we can express the approximation error

$$
e(x ; h):=g(x)-h \sum_{j \in \mathbb{Z}} g(h j) d_{h}(x-h j), \quad x \in \mathbb{R},
$$

for $x \in\left[h j^{*}, h\left(j^{*}+1\right)\right)$ as the finite sum

$$
e(x ; h)=g(x)-h \sum_{j=j^{*}-(L-1)}^{j^{*}+(L-1)} g(h j) d_{h}(x-h j), \quad x \in\left[h j^{*}, h\left(j^{*}+1\right)\right) .
$$

Now, if $g \in B C^{p}(\mathbb{R})$ for some $p>1$, we can express $g(h j)$ with the help of Taylor's theorem in the form

$$
g(h j)=g(x)+\sum_{m=1}^{p-1} \frac{g^{(m)}(x)}{m !}(x-h j)^{m}+\frac{g^{(p)}(\zeta)}{p !}(x-h j)^{p},
$$

for $j^{*}-(L-1) \leq j \leq j^{*}+(L-1)$ with

$$
\zeta \in \begin{cases}(x, x-h j), & j \leq j^{*} \\ (x-h j, x), & j \geq j^{*}\end{cases}
$$

where $g^{(m)}$ represents the $m$-th derivative of $g$. Inserting this into (9.22), using the short hand notation

$$
\sum_{j}:=\sum_{j=j^{*}-(L-1)}^{j^{*}+(L-1)}
$$

yields

$$
\begin{aligned}
e(x ; h)= & g(x)-h \sum_{j} g(h j) d_{h}(x-h j) \\
= & g(x)-g(x) h \sum_{j} d_{h}(x-h j)-\sum_{m=1}^{p-1} \frac{g^{(m)}(x)}{m !} h \sum_{j}(x-h j)^{m} d_{h}(x-h j) \\
& -\frac{g^{(p)}(\zeta)}{p !} \sum_{j}(x-h j)^{p} d_{h}(x-h j), \quad x \in\left[h j^{*}, h\left(j^{*}+1\right)\right) .
\end{aligned}
$$

The first two terms cancel due to (9.20) so that

$$
\begin{aligned}
e(x ; h)=-\sum_{m=1}^{p-1} & \frac{g^{(m)}(x)}{m !} h \sum_{j}(x-h j)^{m} d_{h}(x-h j) \\
& -\frac{g^{(p)}(\zeta)}{p !} \sum_{j}(x-h j)^{p} d_{h}(x-h j), \quad x \in\left[h j^{*}, h\left(j^{*}+1\right)\right) .
\end{aligned}
$$


Now assume in addition that the function $d_{h}$ satisfies the discrete moment conditions

$$
h \sum_{j \in \mathbb{Z}}(x-h j)^{m} d_{h}(x-h j)=\delta_{m 0}, \quad m=0, \ldots, p-1,
$$

where $\delta_{m 0}$ denotes the Kronecker delta. Then it follows from (9.23) and (9.21) that

$$
\begin{aligned}
|e(x ; h)| & =\left|\frac{g^{(p)}(\zeta)}{p !} h \sum_{j}(x-h j)^{p} d_{h}(x-h j)\right| \\
& \leq\left\|g^{(p)}\right\|_{B C\left(I_{j^{*}}\right)}(p !)^{-1} h \sum_{j}|x-h j|^{p}\left|d_{h}(x-h j)\right| \\
& \leq\left\|g^{(p)}\right\|_{B C\left(I_{j^{*}}\right)}(p !)^{-1}(L h)^{p} h \sum_{j}\left|d_{h}(x-h j)\right| \\
& \leq c \frac{L^{p}}{p !}\left\|g^{(p)}\right\|_{B C\left(I_{j^{*}}\right)} h^{p}, \quad x \in\left[h j^{*}, h\left(j^{*}+1\right)\right),
\end{aligned}
$$

where

$$
I_{j^{*}}:=\left[h j^{*}-h(L-1), h j^{*}+h(L-1)\right] .
$$

We summarise the results in the following lemma.

LEMMA 9.4. Let $p \in \mathbb{N}$ and suppose that $d_{h}$ satisfies the discrete moment conditions (9.24) for $m=0, \ldots, p-1$, the finite support condition (9.19) for some $L \in \mathbb{N}$, and the boundedness condition (9.21) for some constant $c>0$. If $g \in B C^{q}\left(I_{j^{*}}\right)$ for $q \geq p$ and $I_{j^{*}}$ given through (9.25), then

$$
\left|g(x)-h \sum_{j \in \mathbb{Z}} g(h j) d_{h}(x-h j)\right| \leq c \frac{L^{p}}{p !}\left\|g^{(p)}\right\|_{B C\left(I_{j^{*}}\right)} h^{p}, \quad x \in\left[h j^{*}, h\left(j^{*}+1\right)\right) .
$$

The discrete moment conditions (9.24) are one of a series of equivalent conditions on the interpolation kernel, known as Strang-Fix conditions, which ensure that (9.16) is an interpolation scheme of order $p$. We now prove a very simple version that shows that a kernel function $d_{h}$ that satisfies the first $p$ discrete moment conditions reproduces all monomials of degree less or equal $p-1$. Depending on the number of discrete moment conditions we denote the kernel functions by $d_{h, p}$.

Lemma 9.5. Let $p \in \mathbb{N}$. Then the following conditions on the functions $d_{h, p}$ are equivalent.

(i) The function $d_{h, p}$ satisfies the first $p$ discrete moment conditions, i.e.

$$
h \sum_{j \in \mathbb{Z}}(x-h j)^{m} d_{h, p}(x-h j)=\delta_{m, 0}, \quad m=0, \ldots, p-1 .
$$


(ii) The function $d_{h, p}$ reproduces all monomials of degree less or equal $p-1$, i.e.

$$
h \sum_{j \in \mathbb{Z}}(h j)^{m} d_{h, p}(x-h j)=x^{m}, \quad m=0, \ldots, p-1 .
$$

Proof. We give a proof for the cases $p=1,2$ and explain the general idea for arbitrary $p>2$.

$\boldsymbol{p}=\mathbf{1}$ : The case $p=1$ is clear.

$\boldsymbol{p}=\mathbf{2}$ : In the case $p=2$, we use the first discrete moment condition

$$
h \sum_{j \in \mathbb{Z}} d_{h, 2}(x-h j)=1
$$

and insert it into the second discrete moment condition

$$
h \sum_{j \in \mathbb{Z}}(x-h j) d_{h, 2}(x-h j)=0
$$

to yield

$$
\begin{aligned}
h \sum_{j \in \mathbb{Z}}(x-h j) d_{h, 2}(x-h j) & =x h \sum_{j \in \mathbb{Z}} d_{h, 2}(x-h j)-h \sum_{j \in \mathbb{Z}}(h j) d_{h, 2}(x-h j) \\
& =x-h \sum_{j \in \mathbb{Z}}(h j) d_{h, 2}(x-h j)=0,
\end{aligned}
$$

which can be written in the equivalent form

$$
h \sum_{j \in \mathbb{Z}}(h j)^{m} d_{h, 2}(x-h j)=x^{m}, \quad m=0,1,
$$

i.e. $d_{h, 2}$ reproduces the monomials $1, x$ exactly.

$\boldsymbol{p}>2$ : For arbitrary $p$ one uses the first discrete moment condition to prove the reproduction of constants by expanding the second discrete moment condition and inserting the first. One continues now in a recursive manner using all of the previous discrete moment conditions and reproduction results. We illustrate the general step.

Assume that $m \leq p$ and that we have shown that $d_{h, p}$ reproduces all monomials $x^{n}$ for $n<m$. Using the binomial theorem to expand the $m$-th discrete moment condition

$$
h \sum_{j \in \mathbb{Z}}(x-h j)^{m} d_{h}(x-h j)=0
$$


yields

$$
\begin{aligned}
h \sum_{j \in \mathbb{Z}}(x-h j)^{m} d_{h}(x-h j)=h \sum_{j \in \mathbb{Z}} \sum_{l=0}^{m}\left(\begin{array}{c}
m \\
l
\end{array}\right) x^{m-l}(-h j)^{l} d_{h}(x-h j) \\
=\sum_{l=0}^{m}(-1)^{l}\left(\begin{array}{c}
m \\
l
\end{array}\right) x^{m-l}\left(h \sum_{j \in \mathbb{Z}}(h j)^{l} d_{h}(x-h j)\right) \\
=\sum_{l=0}^{m-1}(-1)^{l}\left(\begin{array}{c}
m \\
l
\end{array}\right) x^{m-l} x^{l}+(-1)^{m}\left(h \sum_{j \in \mathbb{Z}}(h j)^{m} d_{h}(x-h j)\right) \\
=x^{m} \sum_{l=0}^{m-1}(-1)^{l}\left(\begin{array}{c}
m \\
l
\end{array}\right)+(-1)^{m}\left(h \sum_{j \in \mathbb{Z}}(h j)^{m} d_{h}(x-h j)\right) .
\end{aligned}
$$

With the help of the identity

$$
\sum_{l=0}^{k}(-1)^{l}\left(\begin{array}{l}
n \\
l
\end{array}\right)=(-1)^{k}\left(\begin{array}{c}
n-1 \\
k
\end{array}\right)
$$

for $k=m-1$ and $n=m$ and (9.28) we conclude that

$$
h \sum_{j \in \mathbb{Z}}(x-h j)^{m} d_{h}(x-h j)=(-1)^{m-1} x^{m}+(-1)^{m}\left(h \sum_{j \in \mathbb{Z}}(h j)^{m} d_{h}(x-h j)\right)=0,
$$

which means that $d_{h, p}$ reproduces the monomial $x^{m}$.

We note that, due to the linearity of the interpolation scheme, a interpolation kernel $\delta_{h, p}$ that satisfies the first $p$ discrete moment conditions reproduces polynomials of degree less or equal than $p-1$.

We now give a constructive proof for the existence of a family of interpolatory kernel functions $d_{h, 2 L}$ that reproduces polynomials of degree less or equal than $2 L-1$ and have a support of size $2 L h$. To simplify the presentation we assume that $h=1$ and construct a function $d_{1,2 L}$. The functions $d_{h, 2 L}$ are then given in terms of $d_{1,2 L}$ through

$$
d_{h, 2 L}(x)=\frac{1}{h} d_{1,2 L}(x / h) .
$$

The construction of the interpolation kernels $d_{1,2 L}$ follows an idea presented in [46]. But before we present the general result in LEMMA 9.7, we illustrate the construction process for the case $L=2$.

EXAMPle 9.6. Let $L=2$ and restrict $x$ to $[0,1)$. Then we can write the discrete moment conditions in the equivalent form (9.27), i.e.

$$
\sum_{j=-1}^{2} j^{m} d_{1,4}(x-j)=x^{m}, \quad m=0, \ldots, 3,
$$


which is a system of four equations with the four unknowns $a_{1}(x):=d_{1,4}(x+1)$, $a_{2}(x):=d_{1,4}(x), a_{3}(x):=d_{1,4}(x-1)$ and $a_{4}(x):=d_{1,4}(x-2)$. We write this in matrix form as

$$
\left(\begin{array}{rrrr}
1 & 1 & 1 & 1 \\
-1 & 0 & 1 & 2 \\
1 & 0 & 1 & 4 \\
-1 & 0 & 1 & 8
\end{array}\right)\left(\begin{array}{l}
a_{1}(x) \\
a_{2}(x) \\
a_{3}(x) \\
a_{4}(x)
\end{array}\right)=\left(\begin{array}{l}
1 \\
x \\
x^{2} \\
x^{3}
\end{array}\right), \quad x \in[0,1) .
$$

The matrix is a Vandermonde matrix generated by the vector $(-1,0,1,2)$ and therefore invertible. The solution of this system is given through

$$
\left(\begin{array}{l}
a_{1}(x) \\
a_{2}(x) \\
a_{3}(x) \\
a_{4}(x)
\end{array}\right)=\left(\begin{array}{rrrr}
0 & -\frac{1}{3} & \frac{1}{2} & -\frac{1}{6} \\
1 & -\frac{1}{2} & -1 & \frac{1}{2} \\
0 & 1 & \frac{1}{2} & -\frac{1}{2} \\
0 & -\frac{1}{6} & 0 & \frac{1}{6}
\end{array}\right)\left(\begin{array}{l}
1 \\
x \\
x^{2} \\
x^{3}
\end{array}\right), \quad x \in[0,1)
$$

which we write in the form

$$
\begin{aligned}
& a_{1}(x)=-\frac{1}{3} x+\frac{1}{2} x^{2}-\frac{1}{6} x^{3}=-\frac{1}{6}(x-2)(x-1) x, \quad x \in[1,2] \\
& a_{2}(x)=1-\frac{1}{2} x-x^{2}+\frac{1}{2} x^{3}=\frac{1}{2}(x-2)(x-1)(x+1), \quad x \in[0,1] \\
& a_{3}(x)=x+\frac{1}{2} x^{2}-\frac{1}{2} x^{3}=-\frac{1}{2}(x-2) x(x+1), \quad x \in[-1,0] \\
& a_{4}(x)=-\frac{1}{6} x+\frac{1}{6} x^{3}=\frac{1}{6}(x-1) x(x+1), \quad x \in[-2,-1] .
\end{aligned}
$$

This is not yet the solution, as we have to keep in mind that the above formulas are only valid for $x \in[0,1)$. To determine $d_{1,4}$ on the appropriate intervals we have to make a simple change of variables:

$$
\begin{aligned}
& d_{1,4}(x)= \begin{cases}a_{1}(x-1)=-\frac{1}{6}(x-3)(x-2)(x-1), & 1 \leq x \leq 2 \\
a_{2}(x)=\frac{1}{2}(x-2)(x-1)(x+1), & 0 \leq x<1, \\
a_{3}(x+1)=-\frac{1}{2}(x-1)(x+1)(x+2), & -1 \leq x \leq 0, \\
a_{4}(x+2)=\frac{1}{6}(x+1)(x+2)(x+3), & -2 \leq x \leq-1,\end{cases} \\
&= \begin{cases}\left(1-\frac{x}{3}\right)\left(1-\frac{x}{2}\right)(1-x), & 1 \leq x \leq 2 \\
\left(1-\frac{x}{2}\right)(1-x)(1+x), & 0 \leq x<1, \\
(1-x)(1+x)\left(1+\frac{x}{2}\right), & -1 \leq x \leq 0, \\
(1+x)\left(1+\frac{x}{2}\right)\left(1+\frac{x}{3}\right), & -2 \leq x \leq-1 .\end{cases}
\end{aligned}
$$

As clearly seen the function is symmetric with respect to 0 so that we can write it in the more condensed form

$$
d_{1,4}(x)= \begin{cases}\left(1-\frac{x}{2}\right)(1-x)(1+x), & 0 \leq|x|<1, \\ \left(1-\frac{x}{3}\right)\left(1-\frac{x}{2}\right)(1-x), & 1 \leq|x|<2, \\ 0, & |x| \geq 2\end{cases}
$$


After these preparations the proof of the following lemma is evident.

Lemma 9.7. Let the functions $a_{j}, j=1, \ldots, 2 L$ be given through

$$
\left(\begin{array}{c}
a_{1}(x) \\
\vdots \\
a_{2 L}(x)
\end{array}\right)=V^{-1}\left(\begin{array}{c}
1 \\
\vdots \\
x^{2 L-1}
\end{array}\right)
$$

where $V$ denotes the Vandermonde matrix of size $2 L$ generated by the vector

$$
(-L+1,-L+2, \ldots, L-1, L)
$$

Then the function

$$
d_{1,2 L}(x):= \begin{cases}a_{1}(x-L+1), & L-1 \leq x \leq L, \\ a_{2}(x-L+2), & L-2 \leq x \leq L-1, \\ \vdots & \\ a_{L-1}(x+L-1), & -L+1 \leq x \leq-L+2, \\ a_{L}(x+L), & -L \leq x \leq-L+1,\end{cases}
$$

satisfies the first $2 L$ discrete moment conditions.

The main problem with this lemma is not the question of existence. It is rather the question whether we can understand from the construction process what the functions $a_{j}$ must look like for arbitrary $L$.

We used the computer algebra system MAPLE to compute a representation, the listing of the procedure we wrote is shown in Listing A.1. From these computations we concluded that the function $d_{1,2 L}$ should look like

$$
\Delta_{1,2 L}(x):= \begin{cases}\prod_{\substack{L-1-i \\ m=-L-i \\ m \neq 0}}^{\left.L-i+\frac{x}{m}\right),} & i \leq|x| \leq i+1, \quad i=0, \ldots, L-1, \\ 0, & |x|>L\end{cases}
$$

Illustrations of these kernel functions for $L=2$ and $L=3$ are given in FiguRE 9.2. From our numerical experiments we concluded the following:

Conjecture 9.8. For all $L \in \mathbb{N}$ it holds that $\Delta_{1,2 L}=d_{1,2 L}$.

We verified the conjecture with MAPLE for $1 \leq L \leq 50$, which includes the range of all functions that we used for computations.

REMARK 9.9. The functions $\Delta_{1,2 L}$ seem to have been studied before: 
- A representation of the kernel functions in the monomial basis, together with a MATLAB program to compute the coefficients, is given in [56]. The representation in the monomial basis is, to our understanding, not well suited for an efficient and stable evaluation for large $L$. The representation (9.32) does not suffer from these limitations.

- In the survey article [37, formula (27)] the functions are given in the form (9.32), but without a reference to a general approximation order.

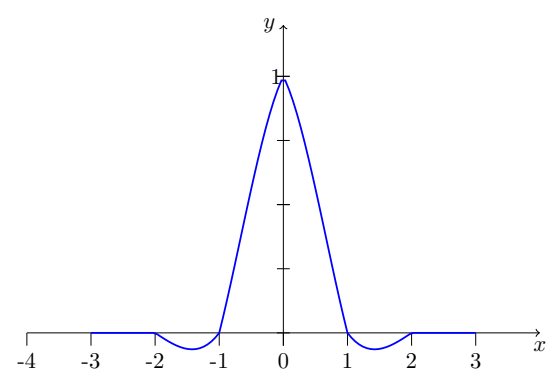

(a) The function $d_{1,4}$.

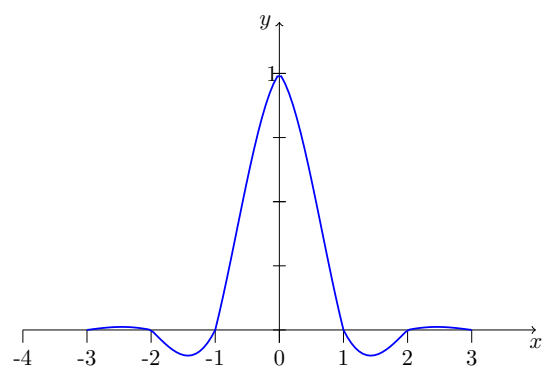

(c) The function $d_{1,6}$.

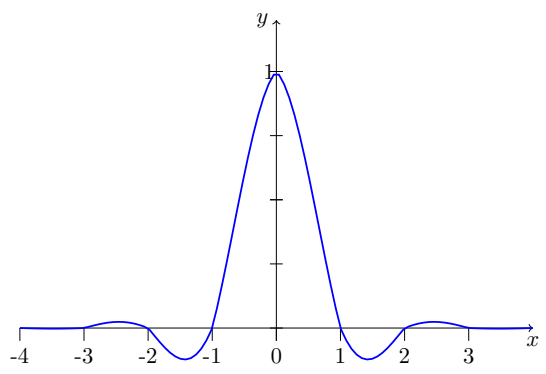

(e) The function $d_{1,8}$.

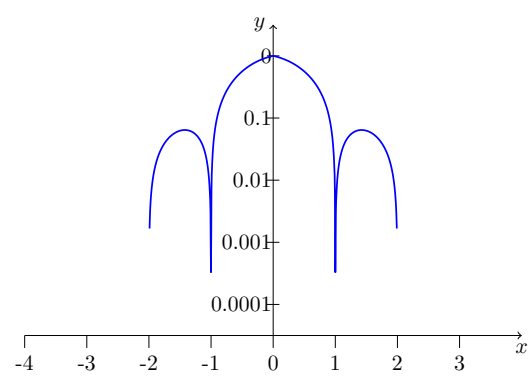

(b) Logarithmic plot of $\left|d_{1,4}\right|$.

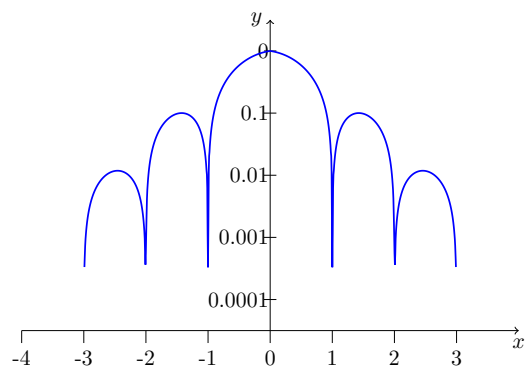

(d) Logarithmic plot of $\left|d_{1,6}\right|$.

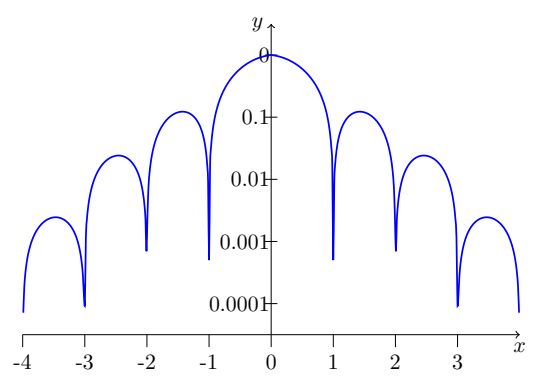

(f) Logarithmic plot of $\left|d_{1,8}\right|$.

FiguRE 9.2: Approximation of the Dirac delta distribution and their logarithmic plot. 


\subsubsection{A high order local interpolation scheme}

From our previous analysis we conclude that the interpolation operator

$$
\left(P_{2 L} g\right)(x):=h \sum_{j \in \mathbb{Z}} g(h j) d_{h, 2 L}(x-h j)
$$

is exact for polynomials up to degree $2 L-1$, which we have proven for $L=1, \ldots, 50$. In analogy to the THEOREM 9.2 we can summarise the results in the next lemma.

LEMmA 9.10. Let $g \in \Pi_{2 L-1}(\mathbb{C})$, the space of complex polynomials with degree at most $2 L-1$, for $L \in \mathbb{N}$. Then $g$ is completely determined by its values $g(h j), j \in \mathbb{Z}$ on a regular grid and

$$
g(x)=\sum_{j \in \mathbb{Z}} g(h j) d_{1,2 L}\left(\frac{x}{h}-j\right), \quad x \in \mathbb{R} .
$$

We can use this Lemma to define a high order local interpolation scheme for smooth functions on $\mathbb{R}$ in the following way:

Lemma 9.11. Let $M \in \mathbb{N}$ and $L \in \mathbb{N}$ with $L<(M+1) / 2$. For real numbers $a, b$ with $a<b$ define the step size

$$
h:=\frac{b-a}{M+1-2 L}
$$

the real numbers

$$
a_{L}:=a-(L-1) h
$$

and

$$
b_{L}:=b+(L-1) h,
$$

and the grid points

$$
x_{j}:=a_{L}+h j, \quad j \in \mathbb{Z} .
$$

Then for all $g \in B C^{p}(\mathbb{R})$ with $p \geq 2 L$ the approximation error satisfies

$$
\left\|g-g_{M, 2 L}\right\|_{B C([a, b])} \leq c_{h, 2 L} \frac{L^{2 L}}{(2 L) !}\left\|g^{(2 L)}\right\|_{B C\left(\left[a_{L}, b_{L}\right]\right)} h^{2 L},
$$

where

$$
g_{M, 2 L}(x):=h \sum_{j \in \mathbb{Z}} g\left(x_{j}\right) d_{h, 2 L}\left(x-x_{j}\right)
$$

and

$$
c_{h, 2 L}:=\sup _{x \in \mathbb{R}} h \sum_{j \in \mathbb{Z}}\left|d_{h, 2 L}(x-h j)\right| .
$$


Proof. The proof follows by applying Lemma 9.4.

We illustrate this local interpolation scheme by the following example.

EXAMPLE 9.12. Consider the function

$$
g(x):=\exp \left(2 \pi i \sqrt{1+x^{2}}\right) / \sqrt{1+x^{2}}
$$

on the interval $I:=[0.25,1.5]$. We note that $g$ possesses a holomorphic extension into the strip $\{z=x+i y:-\infty<x<\infty,-1<y<1\}$, which means that we can expect super-algebraic convergence. The approximation and logarithmic error plots

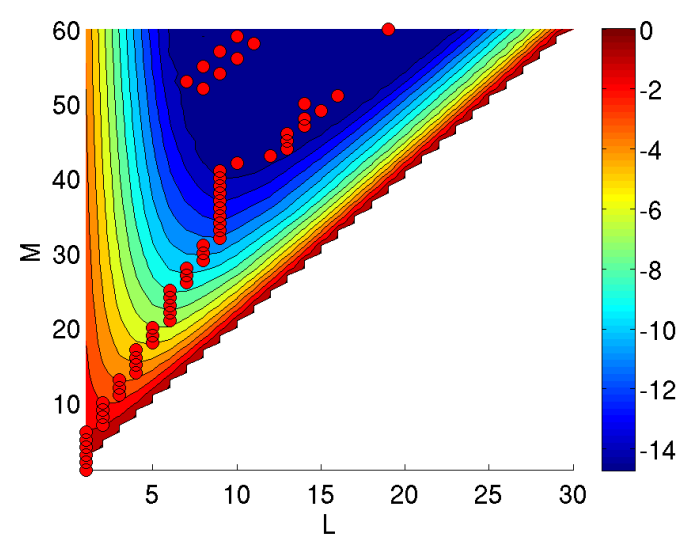

FiguRE 9.3: $\log _{10}\left\|g-g_{M, 2 L}\right\|_{\infty}$

are depicted in FiguRE 9.4 for the values $L=2,5,8$ and $M=30$. In accordance to the error analysis we see that the error inside the interval $I$ is much smaller compared to the error in the extended interval. In FIGURE 9.3 we depicted $\log _{10} \| g-$ $g_{M, 2 L} \|_{\infty}$, i.e. an logarithmic plot of the estimated maximum error inside the interval $I$ in dependence of the order of the interpolation scheme and the total number of interpolation points $M$. Note that, for fixed $M$, the increase in the error for growing $L$ is not some kind of ill-posedeness. It is rather the instance that the step size, for fixed $M$, is an increasing function considered as a function in $L$, as it can be seen from (9.33). The red markers inside the picture denote the approximation order that produced the smallest error for fixed $M$. 


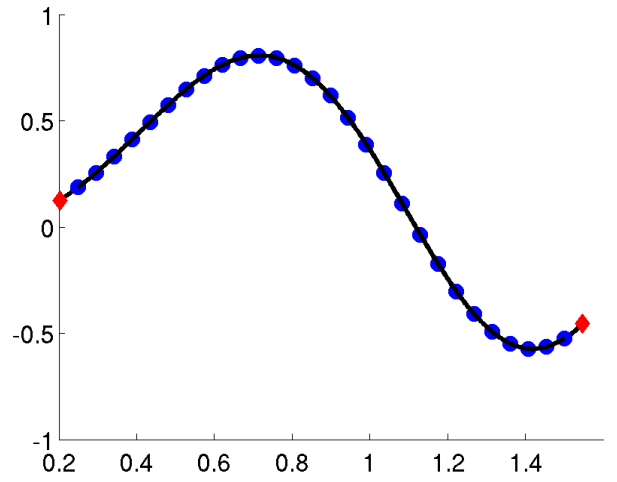

(a) The real part of $g_{30,4}$.

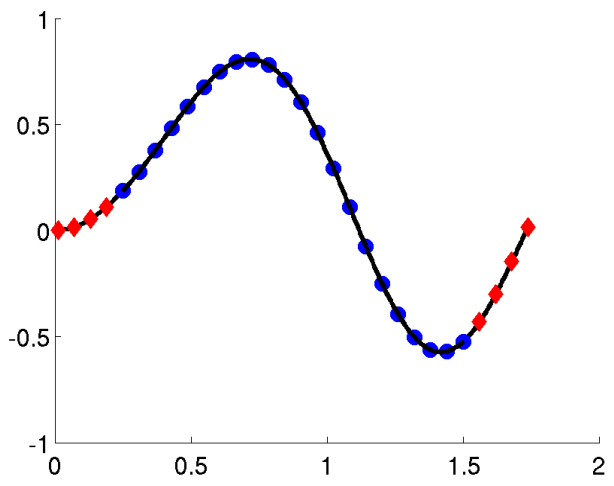

(c) The real part of $g_{30,10}$.

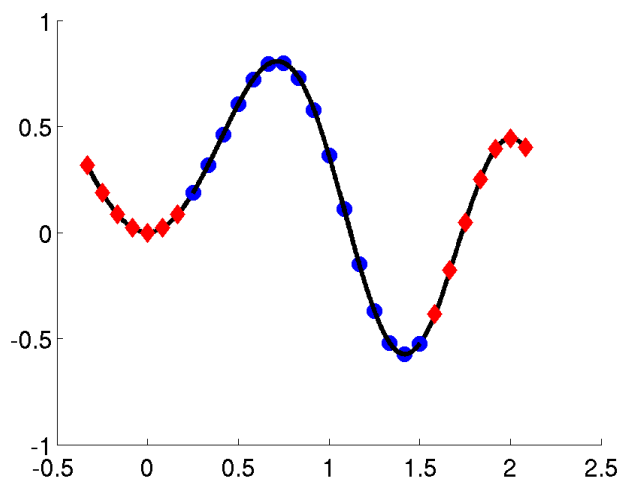

(e) The real part of $g_{30,16}$.

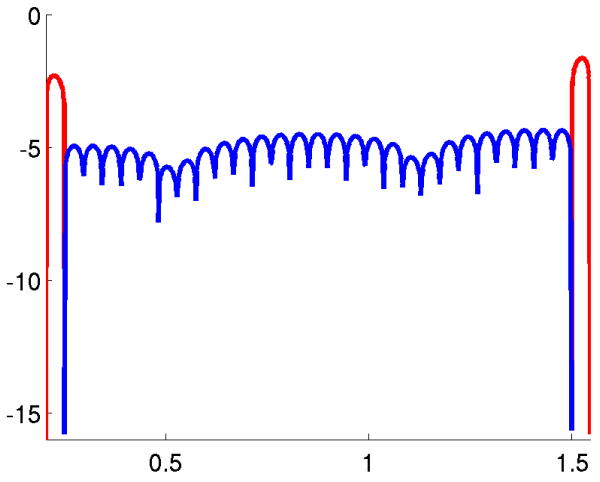

(b) The error for $L=2$.

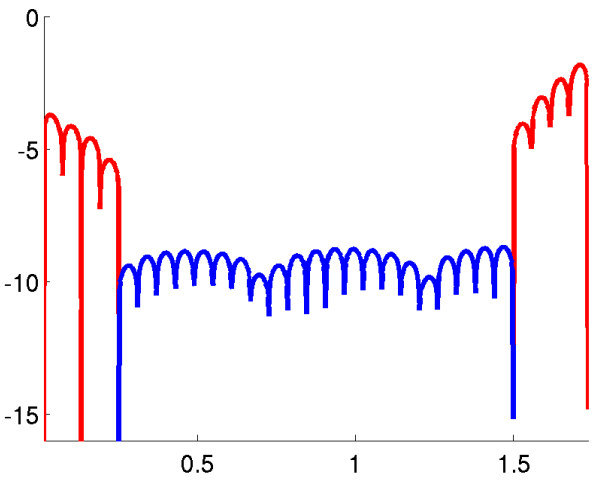

(d) The error for $L=5$.

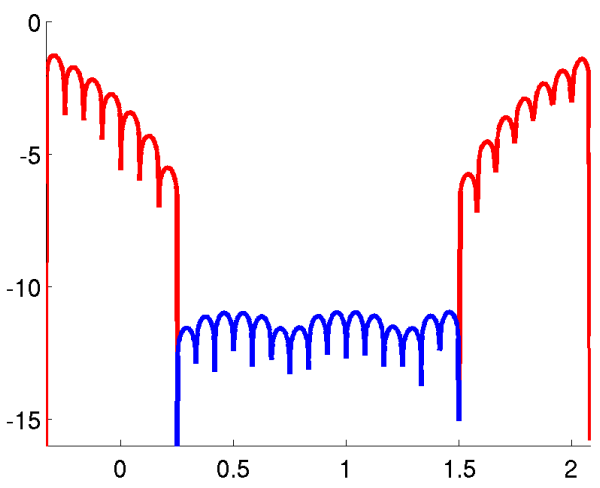

(f) The error for $L=8$.

FiguRE 9.4: The local interpolation scheme for ExAMPLE 9.12. The red diamond shaped markers indicate the points outside the interval of interest that are used for the interpolation scheme. For a scheme of order $2 L$ these are $L-1$ points both on the left and right side of the interval. 


\subsection{Connections to the classical canonical grid method}

We can establish a connection between our novel method as introduced in Section 9.2 and the canonical grid method as introduced before with the help of interpolation operators, cf. (9.7). For this, we combine (9.10) and (9.13) and replace the integrals over $T$ by the composite trapezoidal rule for an equidistant grid. In some more detail: for $M \in \mathbb{N}$ chose $L$ such that the inequality $M>2 L-1$ is satisfied. Now define the step size

$$
h_{3}:=\frac{f_{R}^{+}-f_{R}^{-}}{M+1-2 L}
$$

and use the equidistantly spaced points

$$
x_{j}:=a_{3}+h_{3} j, \quad j=0, \ldots, M-1,
$$

where

$$
a_{3}:=f_{R}^{-}-(L-1) h
$$

For this choice of parameters we get the maximal approximation order for the interpolation scheme of order $2 L$ inside the interval of interest $T$. Due to the smoothness assumption $(\mathbf{C 1})$ we can interchange the order of integration to yield

$$
\begin{aligned}
&(W \psi)(\boldsymbol{x})=\iint_{R} \int_{T} d_{h_{3}, 2 L}\left(f(\boldsymbol{x})-x_{3}\right) k\left(\left(\boldsymbol{x}, x_{3}\right)-\left(\boldsymbol{y}, y_{3}\right)\right) d_{h_{3}, 2 L}\left(f(\boldsymbol{y})-y_{3}\right) d y_{3} d x_{3} \\
& J(\boldsymbol{y}) \psi(\boldsymbol{y}) d \boldsymbol{y} \\
& \approx \sum_{j=0}^{M-1} \sum_{j^{\prime}=0}^{M-1} h_{3} d_{h_{3}, 2 L}\left(f(\boldsymbol{x})-z_{j}\right) \int_{R} k\left(\boldsymbol{x}-\boldsymbol{y}, z_{j}-z_{j^{\prime}}\right) h_{3} d_{h_{3}, 2 L}\left(f(\boldsymbol{y})-z_{j^{\prime}}\right) \\
&=\int_{R} \sum_{j=0}^{M-1} \sum_{j^{\prime}=0}^{M-1} L_{j}(f(\boldsymbol{x})) \psi(\boldsymbol{y}) d \boldsymbol{y}\left(\boldsymbol{x}-\boldsymbol{y}, z_{j-j^{\prime}}\right) L_{j^{\prime}}(f(\boldsymbol{y})) J(\boldsymbol{y}) \psi(\boldsymbol{y}) d \boldsymbol{y},
\end{aligned}
$$

where

$$
L_{j}(\boldsymbol{x}):=h_{3} d_{h, 2 L}\left(\boldsymbol{x}-z_{j}\right), \quad j=0, \ldots, M-1 .
$$

This again is a decomposition of the form (9.3) and the kernel approximation coincides with (9.7). This shows that our novel approach can be implemented as a 2D or 3D variant, cf. Listing 10.2 and Listing 10.1.

A rough estimate for the number of floating point operations and the minimal required memory for the different versions of the matrix-vector multiplication algorithm, for a grid with $N^{*}:=N_{1} \cdot N_{2}$ number of unknowns and $M$ interpolation levels, is summarised in TABLE 9.1. 


\begin{tabular}{|c|c|c|}
\hline & Operation count & Memory \\
\hline $\mathrm{Na}$ & $\left(N^{*}\right)^{2}$ & $N^{*}$ \\
\hline 2D-Alg & $M^{2} \cdot 3 \cdot\left(4 N^{*}\right) \log \left(4 N^{*}\right)$ & $4 N^{*}$ \\
\hline 3D-Alg. & $3 \cdot\left(8 N^{*} M\right) \log \left(8 N^{*} M\right)$ & $8 N^{*} M$ \\
\hline
\end{tabular}

TABLE 9.1: Estimated number of floating point operations and memory usage

\subsection{Applying the method to the rough surface scattering problem}

In order to use the novel method described in Section 9.2 for the rough surface scattering problem, we must apply it to an integral operator of the form

$$
(W \psi)(\boldsymbol{x})=\int_{R} K(\boldsymbol{x}, \boldsymbol{y}) J(\boldsymbol{y}) \psi(\boldsymbol{y}) d \boldsymbol{y}, \quad \boldsymbol{x} \in R,
$$

cf. (9.1), where the kernel function is given through

$$
K(\boldsymbol{x}, \boldsymbol{y})=K_{1}(\boldsymbol{x}, \boldsymbol{y})-K_{2}(\boldsymbol{x}, \boldsymbol{y})-i \eta\left(K_{3}(\boldsymbol{x}, \boldsymbol{y})-K_{4}(\boldsymbol{x}, \boldsymbol{y})\right)
$$

with

$$
\begin{aligned}
K_{1}(\boldsymbol{x}, \boldsymbol{y}) & :=\frac{1}{2 \pi}\left[1-\chi_{a, b}(|\boldsymbol{x}-\boldsymbol{y}|)\right] \frac{\langle\nu(y), x-y\rangle}{|x-y|^{2}}(1-i \kappa|x-y|) \frac{\exp (i \kappa|x-y|)}{|x-y|}, \\
K_{2}(\boldsymbol{x}, \boldsymbol{y}) & :=\frac{1}{2 \pi} \frac{\left\langle[\nu(y)]^{\prime}, x-y^{\prime}\right\rangle}{\left|x-y^{\prime}\right|^{2}}\left(1-i \kappa\left|x-y^{\prime}\right|\right) \frac{\exp \left(i \kappa\left|x-y^{\prime}\right|\right)}{\left|x-y^{\prime}\right|} \\
K_{3}(\boldsymbol{x}, \boldsymbol{y}) & :=\frac{1}{2 \pi}\left(\left[1-\chi_{a, b}(|\boldsymbol{x}-\boldsymbol{y}|)\right] \frac{\cos (\kappa|x-y|)}{|x-y|}+i \frac{\sin (\kappa|x-y|)}{|x-y|}\right), \\
K_{4}(\boldsymbol{x}, \boldsymbol{y}) & :=\frac{1}{2 \pi} \frac{\exp \left(i \kappa\left|x-y^{\prime}\right|\right)}{\left|x-y^{\prime}\right|}
\end{aligned}
$$

where

$$
x-y=(\boldsymbol{x}-\boldsymbol{y}, f(\boldsymbol{x})-f(\boldsymbol{y})) \quad \text { and } \quad x-y^{\prime}=(\boldsymbol{x}-\boldsymbol{y}, f(\boldsymbol{x})+f(\boldsymbol{y})) .
$$

We note that the kernel $K_{3}$ can be written as

$$
K_{3}(\boldsymbol{x}, \boldsymbol{y})=k_{3}(\boldsymbol{x}-\boldsymbol{y}, f(\boldsymbol{x})-f(\boldsymbol{y})),
$$

where

$$
k_{3}(z):=\frac{1}{2 \pi}\left(\left[1-\chi_{a, b}(|\boldsymbol{z}|)\right] \frac{\cos (\kappa|z|)}{|z|}+i \frac{\sin (\kappa|z|)}{|z|}\right), \quad z=\left(\boldsymbol{z}, z_{3}\right) \in \mathbb{R}^{3} .
$$


The kernel $K_{1}$ can be written in a similar way as

$$
K_{1}(\boldsymbol{x}, \boldsymbol{y})=\sum_{l=1}^{3} k_{1, l}(\boldsymbol{x}-\boldsymbol{y}, f(\boldsymbol{x})-f(\boldsymbol{y})) c_{l}(\boldsymbol{y}),
$$

where

$$
k_{1, l}(z):=\frac{1}{2 \pi}\left[1-\chi_{a, b}(|\boldsymbol{z}|)\right] \frac{z_{l}}{|z|^{2}}(1-i \kappa|z|) \frac{\exp (i \kappa|z|)}{|z|}, \quad z=\left(\boldsymbol{z}, z_{3}\right) \in \mathbb{R}^{3}
$$

and

$$
c_{l}(\boldsymbol{z}):=\nu_{l}(\boldsymbol{z}, f(\boldsymbol{z}))= \begin{cases}-\partial_{l} f(\boldsymbol{y})\left(1+|\nabla f(\boldsymbol{z})|^{2}\right)^{-1 / 2}, & l=1,2, \\ \left(1+|\nabla f(\boldsymbol{z})|^{2}\right)^{-1 / 2}, & l=3 .\end{cases}
$$

Thus we can apply the method to each component individually.

The kernel $K_{4}$ can be written in the form

$$
K_{4}(\boldsymbol{x}, \boldsymbol{y})=k_{4}(\boldsymbol{x}-\boldsymbol{y}, f(\boldsymbol{x})+f(\boldsymbol{y}))
$$

where

$$
k_{4}(z):=\frac{1}{2 \pi} \frac{\exp (i \kappa|z|)}{|z|}, \quad z=\left(\boldsymbol{z}, z_{3}\right) \in \mathbb{R}^{3} .
$$

The kernel $K_{2}$ can be written in a similar way as

$$
K_{2}(\boldsymbol{x}, \boldsymbol{y})=\sum_{l=1}^{3} k_{2, l}(\boldsymbol{x}-\boldsymbol{y}, f(\boldsymbol{x})+f(\boldsymbol{y})) d_{l}(\boldsymbol{y}),
$$

where

$$
k_{2, l}(z):=\frac{1}{2 \pi} \frac{z_{l}}{|z|^{2}}(1-i \kappa|z|) \frac{\exp (i \kappa|z|)}{|z|}, \quad z=\left(\boldsymbol{z}, z_{3}\right) \in \mathbb{R}^{3}
$$

and

$$
d_{l}(\boldsymbol{z}):=\left[\nu_{l}(\boldsymbol{z}, f(\boldsymbol{z}))\right]^{\prime}= \begin{cases}-\partial_{l} f(\boldsymbol{y})\left(1+|\nabla f(\boldsymbol{z})|^{2}\right)^{-1 / 2}, & l=1,2 \\ -\left(1+|\nabla f(\boldsymbol{z})|^{2}\right)^{-1 / 2}, & l=3 .\end{cases}
$$

\subsubsection{Extending the fast method to certain non-difference kernels}

From the above considerations we see that, in order to apply the fast method to the operators arising from the rough surface scattering problem, we need to extend our method to kernel functions of the form

$$
K(\boldsymbol{x}, \boldsymbol{y})=k(\boldsymbol{x}-\boldsymbol{y}, f(\boldsymbol{x})+f(\boldsymbol{y})) .
$$


To handle these kind of kernel functions we introduce the shifted kernel

$$
\tilde{k}\left(\boldsymbol{x}, x_{3}\right):=k\left(\boldsymbol{x}, x_{3}+b_{3}+a_{3}\right) .
$$

As before we start with the associated cube potential

$$
(\mathcal{W} \psi)(x)=\int_{R} k(x-(\boldsymbol{y},-f(\boldsymbol{y}))) J(\boldsymbol{y}) \psi(\boldsymbol{y}) d \boldsymbol{y}, \quad x=\left(\boldsymbol{x}, x_{3}\right) \in Q .
$$

Using the shifted kernel $\tilde{k}$ and shifted Dirac delta distribution we can write the cube potential in the form

$$
\begin{aligned}
(\mathcal{W} \psi)(x) & =\int_{R} k(x-(\boldsymbol{y},-f(\boldsymbol{y}))) J(\boldsymbol{y}) \psi(\boldsymbol{y}) d \boldsymbol{y} \\
& =\int_{R} \tilde{k}\left(x-\left(\boldsymbol{y}, b_{3}+a_{3}-f(\boldsymbol{y})\right)\right) J(\boldsymbol{y}) \psi(\boldsymbol{y}) d \boldsymbol{y} \\
& =\int_{R} \int_{T} \tilde{k}\left(x-\left(\boldsymbol{y}, b_{3}+a_{3}-f(\boldsymbol{y})\right)\right) J(\boldsymbol{y}) \psi(\boldsymbol{y}) \delta\left(\left[b_{3}+a_{3}-f(\boldsymbol{y})\right]-y_{3}\right) d y_{3} d \boldsymbol{y} \\
& =\int_{Q} \tilde{k}(x-y) \Psi(y) d y, \quad x \in Q,
\end{aligned}
$$

where

$$
\Psi(y):=J(\boldsymbol{y}) \psi(\boldsymbol{y}) \delta\left(\left[b_{a}+a_{3}-f(\boldsymbol{y})\right]-y_{3}\right), \quad y=\left(\boldsymbol{y}, y_{3}\right) \in Q .
$$

The second step can be handled as before, i.e.

$$
(W \psi)(\boldsymbol{x})=\int_{T} \delta\left(f(\boldsymbol{x})-x_{3}\right)(\mathcal{W} \psi)\left(\boldsymbol{x}, x_{3}\right) d x_{3}, \quad \boldsymbol{x} \in R .
$$

\subsection{Alternative kernel functions}

In the excellent reviews and survey articles [37], [54] and [8] a large family of interpolation kernels are examined. The methods described in the articles include the use of radial basis functions. For more information we refer the reader to the monographs [10] and [59] and the citations therein.

\subsubsection{Radial basis functions}

We now explain some ideas for an high order interpolation scheme that is based on the use of radial basis functions. The use of RBF for the interpolation scheme was suggested by Prof. Schaback, whom I am indebted to for discussions and many useful hints.

Let $\Phi$ be a radial function, i.e. there exists a function $\phi: \mathbb{R}_{\geq 0} \rightarrow \mathbb{C}$ such that

$$
\Phi(x)=\phi(|x|), \quad x \in \mathbb{R} .
$$


Then we try to approximate a function $g$ on an interval $[a, b]$ by the finite sum

$$
\sum_{j=0}^{M-1} g\left(x_{j}\right) u_{j}^{*}(x), \quad x \in[a, b],
$$

where $x_{j}$, for $j=0, \ldots, M-1$, are pairwise distinct points inside $[a, b]$ and the functions $u_{j}^{*}$, for $j=0, \ldots, M-1$, are given as a weighted sum of the radial basis function $\Phi$, i.e.

$$
u_{j}^{*}(x):=\sum_{k=0}^{M-1} \alpha_{j, k} \Phi\left(x-x_{k}\right) .
$$

We require that the functions $u_{j}^{*}$ satisfy the interpolation property

$$
u_{j}^{*}\left(x_{i}\right)=\delta_{i, j}, \quad i, j=0, \ldots, M-1 .
$$

Thus the coefficients $\alpha_{j}=\left(\alpha_{j, 0}, \ldots, \alpha_{j, M-1}\right)^{t}$ of the functions $u_{j}^{*}$ are given as the solution of the linear system

$$
\sum_{k=0}^{M-1} \Phi\left(x_{i}-x_{k}\right) \alpha_{j, k}=\delta_{i, j}, \quad i=0, \ldots, M .
$$

We can write this as a matrix equation

$$
D A=I,
$$

where the two $M \times M$ matrices $D$ and $A$ are given through

$$
\begin{aligned}
(D)_{i, k} & :=\Phi\left(x_{i}-x_{k}\right), \quad i, k=0, \ldots, M \\
(A)_{i, k} & :=\alpha_{i, k}, \quad i, k=0, \ldots, M,
\end{aligned}
$$

and $I$ denotes the identity matrix in $\mathbb{R}^{n}$. Using the radial symmetry of $\Phi$ we see that

$$
\Phi\left(x_{i}-x_{k}\right)=\phi\left(\left|x_{i}-x_{k}\right|\right)=\phi\left(\left|x_{k}-x_{i}\right|\right)=\Phi\left(x_{k}-x_{i}\right),
$$

i.e. the kernel matrix $D$ is, by construction, symmetric. For certain classes of functions $\Phi$, one can show that the matrix $D$ is positive definite and hence invertible.

To find a criteria, whether a given function $\phi$ produces an invertible matrix $D$ one defines:

Definition 9.13. A continuous function $\Phi: \mathbb{R} \rightarrow \mathbb{C}$ is called positive definite if, for all $M \in \mathbb{N}$ and all sets of pairwise distinct points $X=\left\{x_{0}, \ldots, x_{M-1}\right\} \subset \mathbb{R}$ the kernel matrix $D$ is positive definite, i.e. for all $\alpha \in \mathbb{C}^{M}$, the quadratic form

$$
\sum_{j=0}^{M-1} \sum_{k=0}^{M-1} \alpha_{j} \bar{\alpha}_{k} \Phi\left(x_{j}-x_{k}\right)
$$

is positive. 
The functions

$$
\phi_{\gamma}(x):=e^{-\gamma|x|^{2}}, \quad \gamma>0, \quad x \in \mathbb{R},
$$

are positive definite, cf. [59, Theorem 6.10]. Though the matrix equation (9.52) is always invertible, the matrix $D$ can be very ill-conditioned. In general, the condition number is a function of the separation distance

$$
S([a, b]):=\frac{1}{2} \min _{0 \leq i<j \leq M-1}\left|x_{i}-x_{j}\right|
$$

that grows dramatically for $S([a, b]) \rightarrow 0$. We use an equidistantly spaced grid with step size $h$, thus $h=2 S([a, b])$.

To control the condition of the kernel matrix $D$ we use the scaling parameter $\gamma$. From a practical point of view it is o.k. to work with condition numbers up to $10^{7}-10^{10}$ and still compute reliable results. In addition to the parameter $\gamma$ we introduced a very simple form of a regularisation scheme, i.e. instead of solving (9.52) we solved the system

$$
(D+\varepsilon I) A=I, \quad \varepsilon>0
$$

for some small values of $\varepsilon$.

\subsection{Numerical results}

All the results shown in this section have been carried out for the kernel function

$$
K_{3}(\boldsymbol{x}, \boldsymbol{y})=k_{3}(\boldsymbol{x}-\boldsymbol{y}, f(\boldsymbol{x})-f(\boldsymbol{y})),
$$

where

$$
k_{3}(z)=\frac{1}{2 \pi}\left(\left[1-\chi_{a, b}(|\boldsymbol{z}|)\right] \frac{\cos (\kappa|z|)}{|z|}+i \frac{\sin (\kappa|z|)}{|z|}\right), \quad z=\left(\boldsymbol{z}, z_{3}\right) \in \mathbb{R}^{3} .
$$

This kernel is one part of the global kernel $k_{\text {global }}^{S}$ of the single-layer potential. We note that the function $z_{3} \mapsto k_{3}\left(\boldsymbol{z}, z_{3}\right)$ possesses a holomorphic extension into the strip

$$
\{z=x+i y:-\infty<x<\infty,-a<y<a\},
$$

i.e. the width of the strip is determined by the width of the support of the function $1-\chi_{a, b}(|\cdot|)$. Thus we can expect super-algebraic convergence. We like to mention that the resemblance of FiguRE 9.7(f) with FigurE 9.3 is due to the fact that both plots show almost the same function, namely FIGURE 9.3 shows the error plot for the function $z_{3} \mapsto k_{3}\left(\boldsymbol{z}, z_{3}\right)$ for a fixed $\boldsymbol{z} \in \mathbb{R}^{2}$ with $|\boldsymbol{z}|=1$, whereas FiguRE 9.7(f) shows the maximal error for $\boldsymbol{z} \in R$. 
The surface height function used in all of the following example is depicted in FiguRE 9.5. It is a two-dimensional trigonometric polynomial of degree $(20,20)$ that can be seen as a realisation of a random rough surface. The supporting rectangle of the surface patch is $R=[-10,10] \times[-10,10]$ and the maximal and minimal values of the surface height function were estimated with $f_{R}^{-}=2.1$ and $f_{R}^{+}=3.9$.

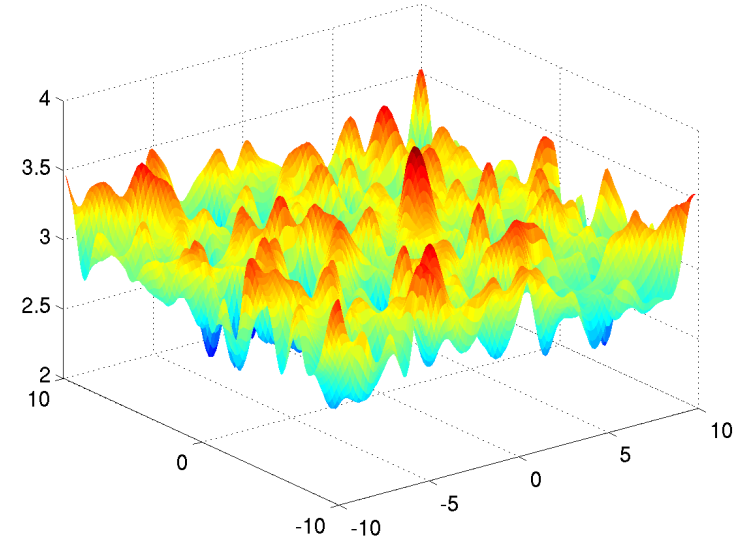

FiguRE 9.5: Realisation of a random rough surface

We used a wavenumber $\kappa=2 \pi$ so that the wavelength $\lambda=\kappa / 2 \pi$ is 1 . Thus, in terms of the wavelength, the surface elevation is $2 \lambda$ and the supporting rectangle has size $20 \lambda \times 20 \lambda$.

For a given density $\psi$ we are interested in understanding the discrete relative $L^{2}$-error

$$
e(L, M):=\frac{\left\|A \psi-A^{(M, L)} \psi\right\|_{L^{2}}}{\|A \psi\|_{L^{2}}}
$$

as a function of the number of expansion levels $M$, used for the interpolation scheme, and the connection to the interpolation order $2 L$. The matrix $A$ represents the fully discrete version of the integral operator and $A^{(M, L)}$ the approximated matrix. The real part of the density $\psi$ and $A \psi$ are shown in FIgURE 9.6, for which we used a two dimensional array of $128 \times 128$ points.

The Figure 9.7(a) shows a logarithmic plot of the discrete relative $L^{2}$-error for $1 \leq M \leq 50$ and $1 \leq L<\frac{M+1}{2}$. The red markers inside this figure denote the function

$$
e_{\min , L}: M \mapsto \min _{L} e(L, M),
$$

i.e. the minimal error that was achieved among all interpolation schemes for fixed $M$. The excellent convergence results are clearly notable and shown again in FIGURE $9.7(b)$

FIGURE 9.7(c)-(d) shows the error plots for the interpolation scheme based on radial basis functions. The relative $L^{2}$-error now depends on $M$ and the scaling 


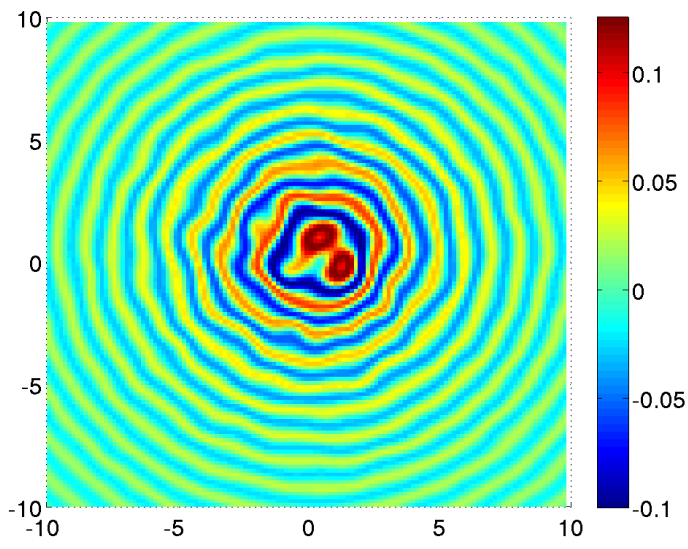

(a) $\Re(\psi)$

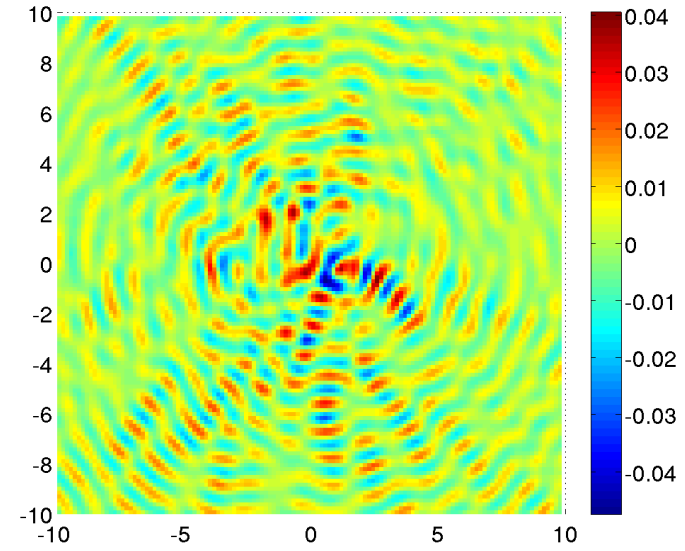

(b) $\Re(A \psi)$

Figure 9.6: The real part of the density $\psi$ and $A \psi$ used to compute the relative $L^{2}$-error.

parameter $\gamma$. The plots show the error for the values $4 \leq M \leq 16$ and different values of $\gamma \in[1,4]$. The red markers show again the minimal error for fixed $M$. The ill-conditioning of the kernel matrix $D$, as mentioned before, can be seen in Figure 9.7(f). We used the rather simple regularisation scheme (9.54) for the choice $\varepsilon=10^{-12}$ for the computations. More sophisticated regularisation schemes with a strategy for the choice of a regularisation parameter might be able to produce even higher accuracy, though even this simple scheme produced results comparible to the high order interpolation scheme, as can be seen in FigurE 9.7(e).

We like to note that the two scheme complement each other for different ranges of accuracies and amount of work. If we keep in mind that we want to accelerate the matrix-vector multiplication in an iterative linear solver, it does not seem reasonable to invest to much work and time into a highly accurate matrix-vector multiplication. Thus an approximation of the matrix-vector product with a relative error of $10^{-4}$ $10^{5}$ will surely be enough. From the error plots we see that 10 points for the RBF method and 20 points for the method with interpolation kernel $d_{1,2 L}$ are sufficient. 


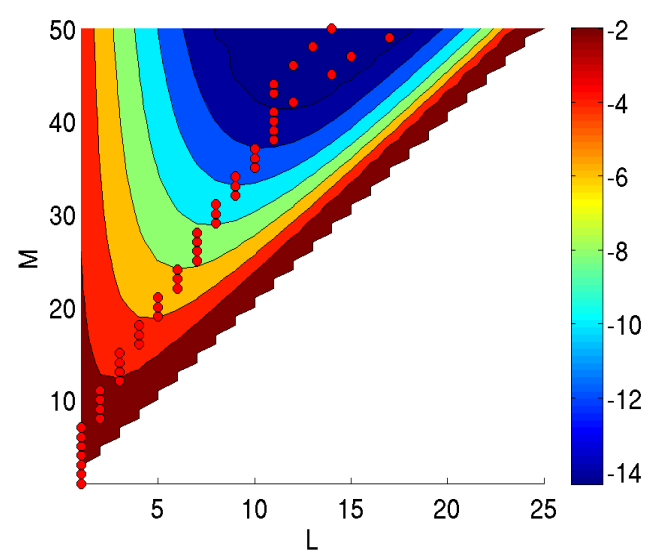

(a) Logarithmic plot of $(L, M) \mapsto e(L, M)$

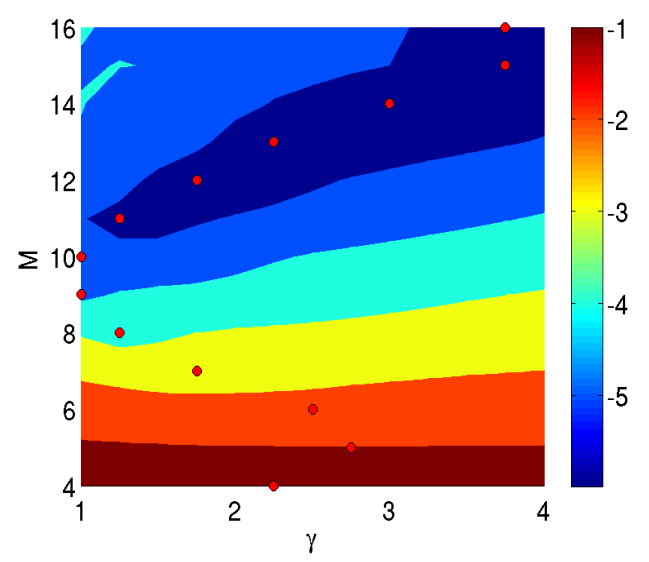

(c) Logarithmic plot of $(\gamma, M) \mapsto e(\gamma, M)$

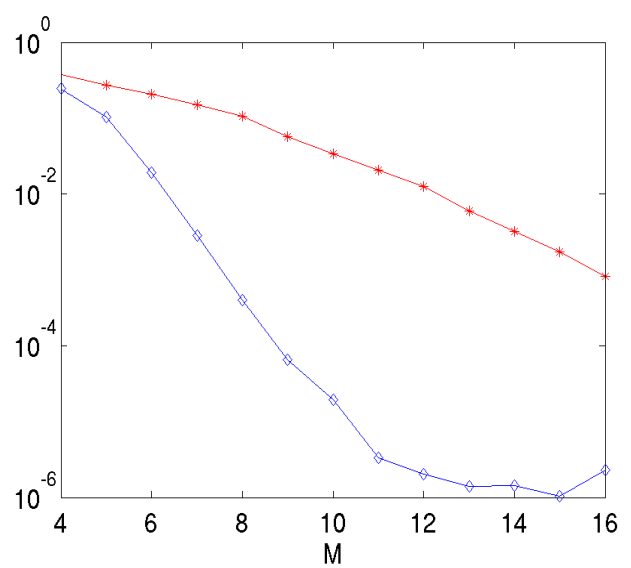

(e) Comparing $e_{\min , L}$ and $e_{\min , \gamma}$

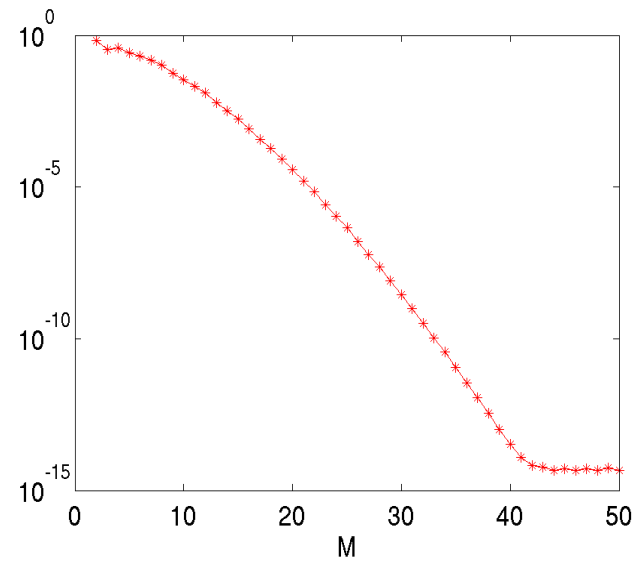

(b) Logarithmic plot of $e_{\min , L}$

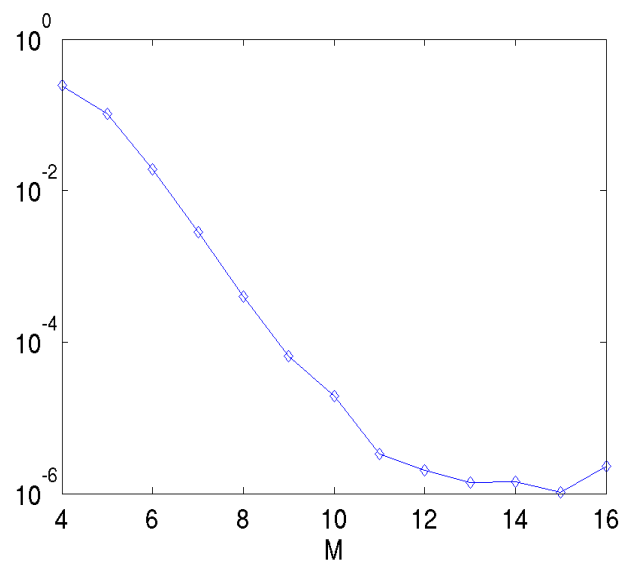

(d) Logarithmic plot of $e_{\min , \gamma}$

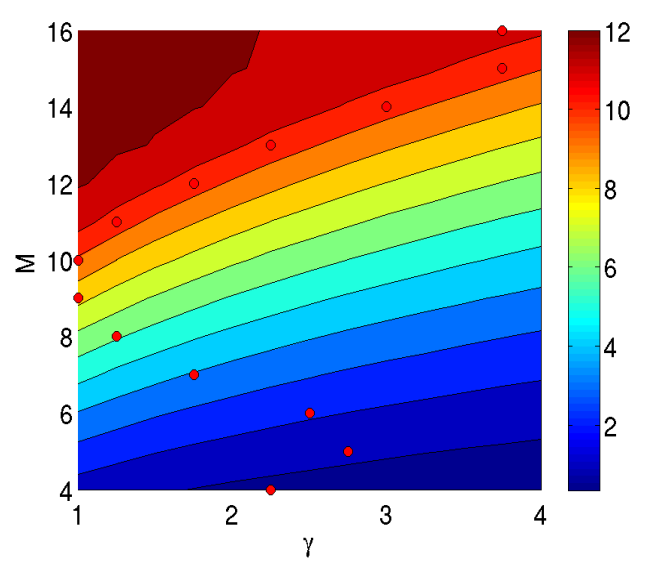

(f) Condition of the kernel matrix $D$

FIgURE 9.7: Error plots for the FMVM, using the interpolation kernel $d_{1,2 L}$ and $\phi_{\gamma}$. 


\section{Chapter 10}

\section{Fast method - Implementation details}

In this chapter we present the details of the fast algorithm on the full discrete level.

We think that the algorithm is conceptually rather simple and therefore easy to implement though at the same time highly efficient for the underlying problem. In fact, we give an example of a MATLAB implementation of the main algorithm in less than a page of code.

The main algorithm of the novel fast matrix-vector multiplication is implemented in the MATLAB function FMVM (fast matrix-vector multiplication) shown in LISTING 10.1. The function my_kernel (cf. Listing 10.3) and chi (cf. Listing 10.4) show an implementation of the kernel function $k_{3}$ used for the numerical example and the cut off function $\chi_{a, b}$. The function FMVM_2D shown in LisTing 10.2 is our implementation of the novel interpolation scheme in a 2D version. Our implementation of the interpolation kernels $d_{h, 2 L}$ is shown in LISTING 10.5 and named DDD for discrete Dirac delta function.

A typical calling sequence could look like follows.

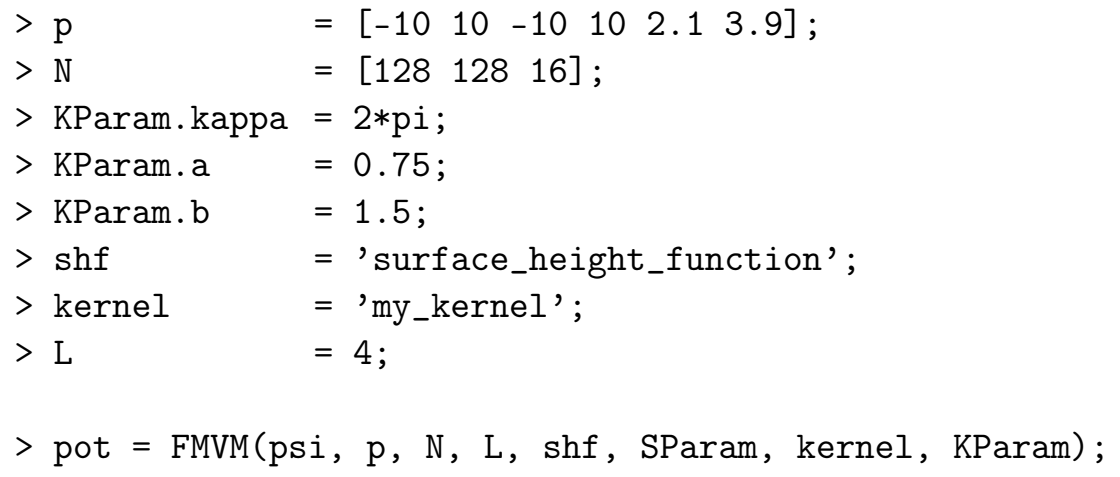

The function expects 8 values:

psi A two-dimensional array that represents the vector in the matrix-vector multiplication.

$\mathrm{p}$ A column vector $\mathrm{p}=[\mathrm{a} 1 \mathrm{~b} 1 \mathrm{a} 2 \mathrm{~b} 2 \mathrm{fmin}$ fmax $]$ that describes the smallest cube that contains the surface patch $\Gamma_{R}$ with supporting rectangle $R$, where 
$R=[\mathrm{a} 1, \mathrm{~b} 1] \times[\mathrm{a} 2, \mathrm{~b} 2]$ and $\mathrm{fmin}$ and $\mathrm{fmax}$ are the minimum and maximum of the surface height function $f$ on $R$.

$\mathrm{N}$ A column vector $\mathrm{N}=\left[\begin{array}{lll}\mathrm{N} 1 & \mathrm{~N} 2 & \mathrm{M}\end{array}\right]$ that contains the number of points, which are used for the discretisation of each space dimension, i.e. in the above example we use 128 points each for the $x_{1}$ and $x_{2}$ direction and 16 points for the discretisation in $x_{3}$ direction.

L A positive integer number that defines the order $(2 L)$ of the local interpolation scheme.

shf A string with the name of a user defined matlab file that contains an implementation of the surface height function. The function can use parameters, contained in the structure SParam, that are used to describe the surface.

SParam A structure that contains parameters that are needed to describe the surface height function.

kernel A string with the name of a user defined matlab file that contains the kernel function. The function can use parameters, contained in the structure KParam.

KParam A structure that contains parameters for the kernel function, e.g. the wave number $\kappa$ or the cut-off parameters $a$ and $b$ for the function $\chi_{a, b}$.

The matlab function my_kernel shown in LisTiNG 10.3 is an implementation of the kernel $K_{3}$ from the numerical examples.

The implementation shown in LISTING 10.1 is not efficient on the use of computer memory, but even this version can be used to compute problems of the size $512 \times$ $512 \times 32$ on machines with up to $4 \mathrm{~GB}$ of RAM.

We now describe the implementation almost line by line and draw the connection to the formulas in the previous sections:

1-5 Define some abbreviations.

7-10 Setup the surface grid and the surface area element $J$.

12-14 Setup the enlarged interval for the interpolation in $x_{3}$-direction, cf. (9.33) and (9.34).

15-18 Setup the 3D grid inside $Q$ and evaluate the the Dirac delta distribution.

19 The function $\Psi$ on the discrete grid inside $Q$, cf. (9.12).

21-24 Setup the large grid to evaluate the periodised version of the kernel function.

27 Use the discrete form of the convolution theorem, cf.(8.5), to compute the action of the operator on the density. 
28 Restrict the solution to the grid points inside $Q$.

29 Use (9.13) to compute the discrete form of $(W \psi)(\boldsymbol{x})$ on the surface grid.

$\overline{\text { LISTING 10.1 MATLAB implementation of the fast matrix-vector multiplication }}$ algorithm.

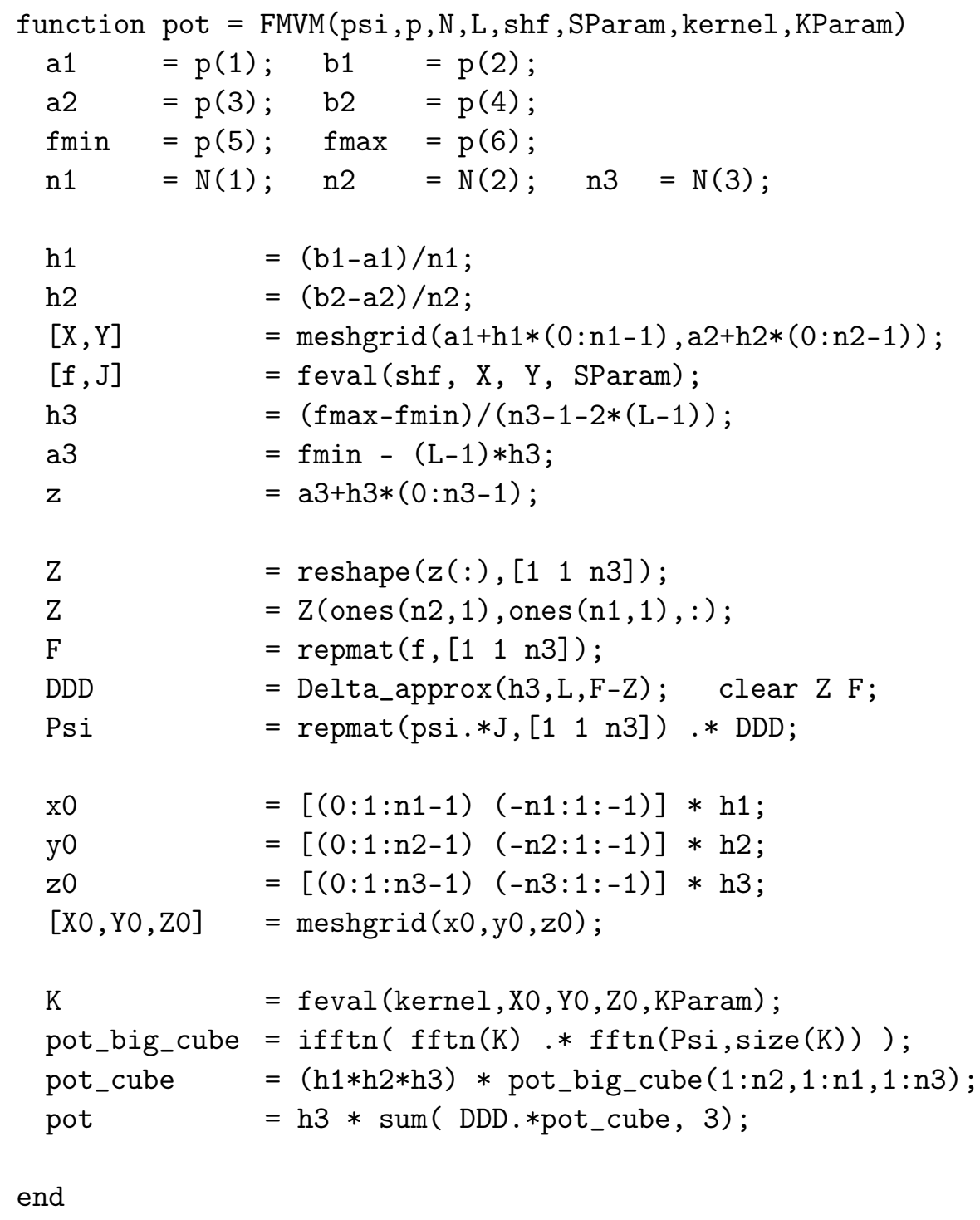


$\overline{\text { LISTING 10.2 MATLAB implementation of the fast matrix-vector multiplication }}$ algorithm in a $2 \mathrm{D}$-variant.

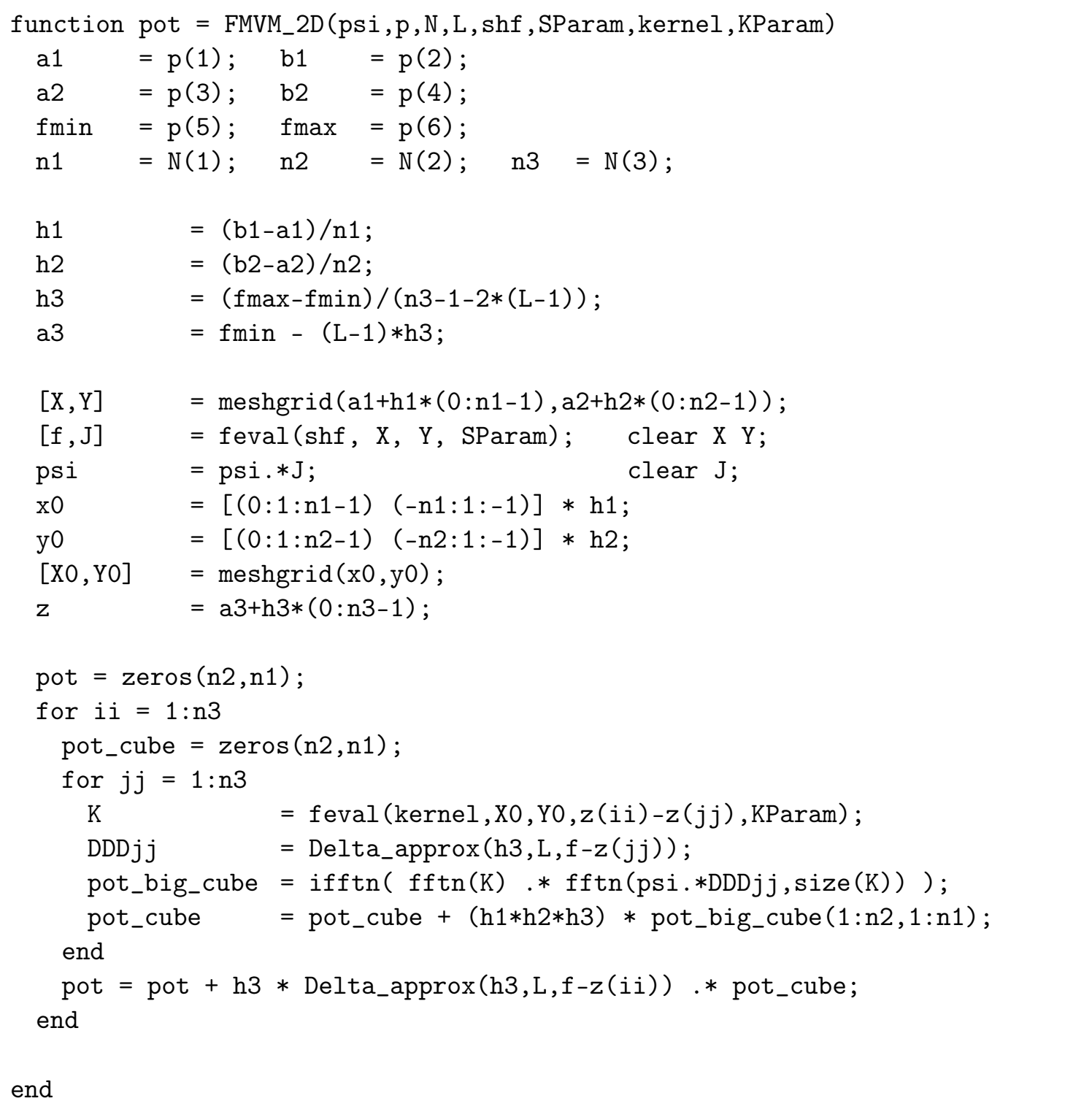



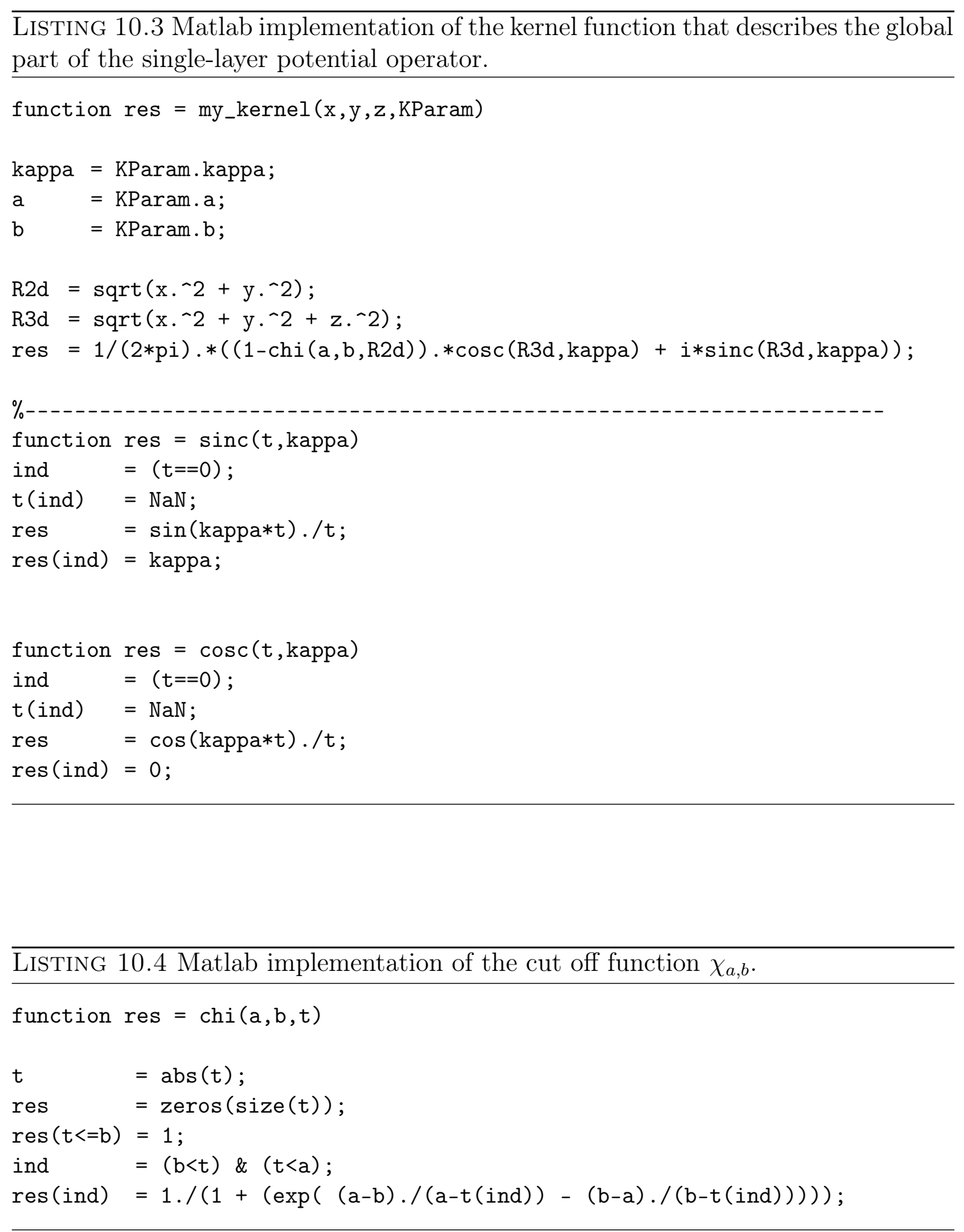


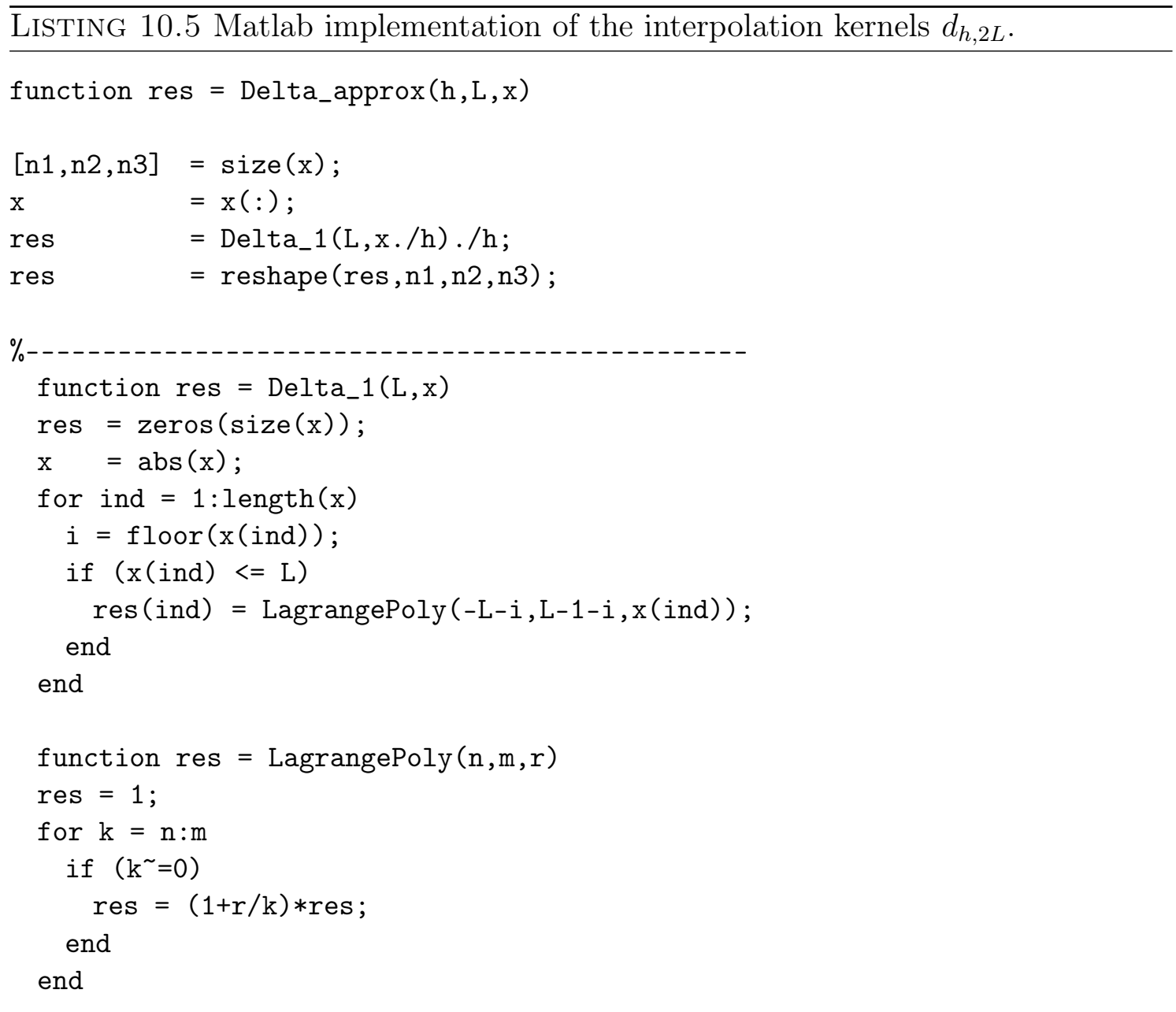




\section{Appendix A}

\section{Maple Procedures}

This chapter contains the Maple procedures DDA, cf. LISTING A.1, and DDA2, cf. LISTING A.2, that were used to compute representations of the kernel function $d_{1,2 L}$ for arbitrary $L$ and to verify the CONJECTURE 9.8 .

LISTING A.1 Maple procedure that computes the function $d_{1,2 L}$ by solving the system of discrete moments equations. The function $d_{1,2 L}(t)$ is computed through the command DDA (L) (t).

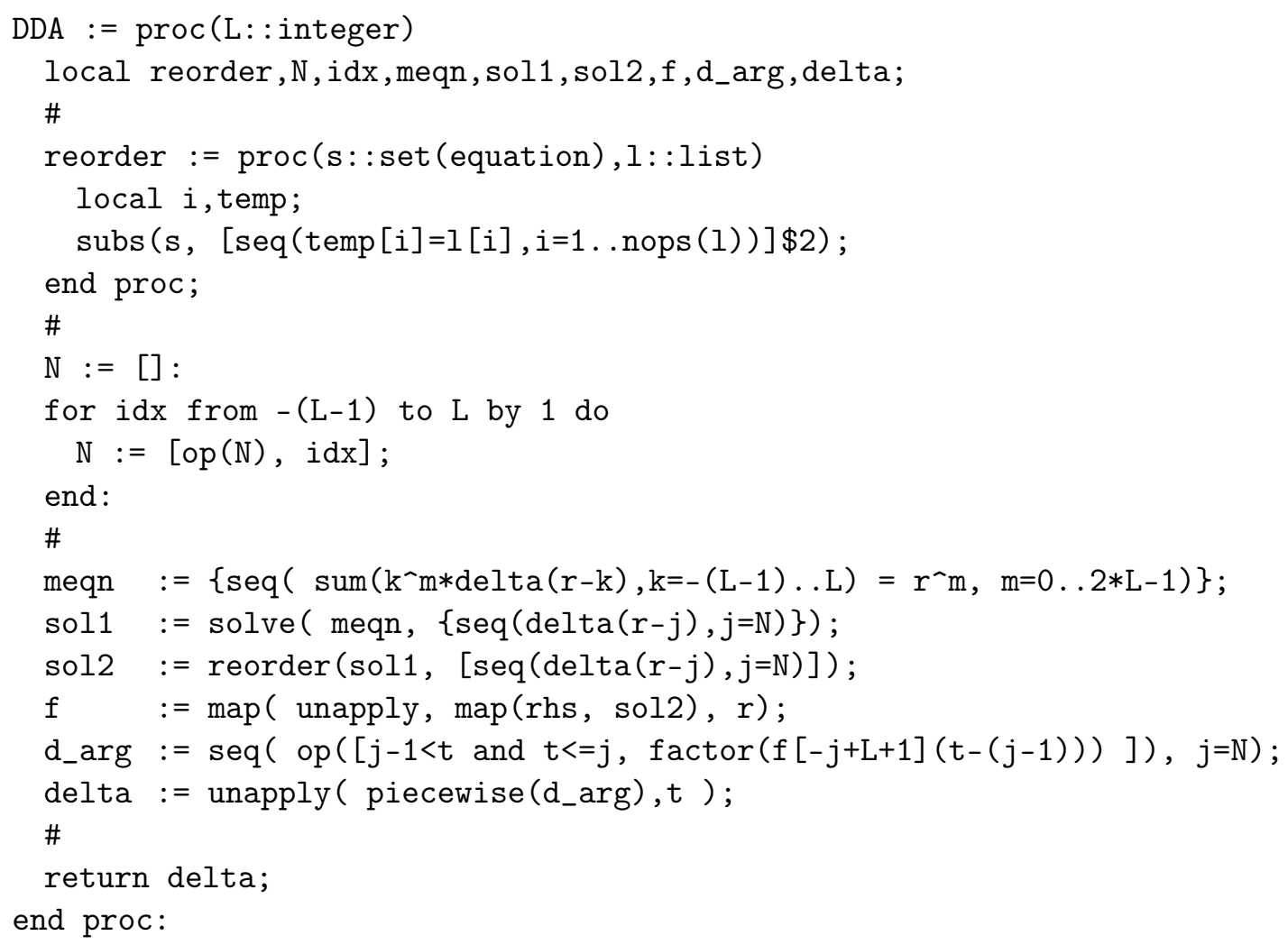




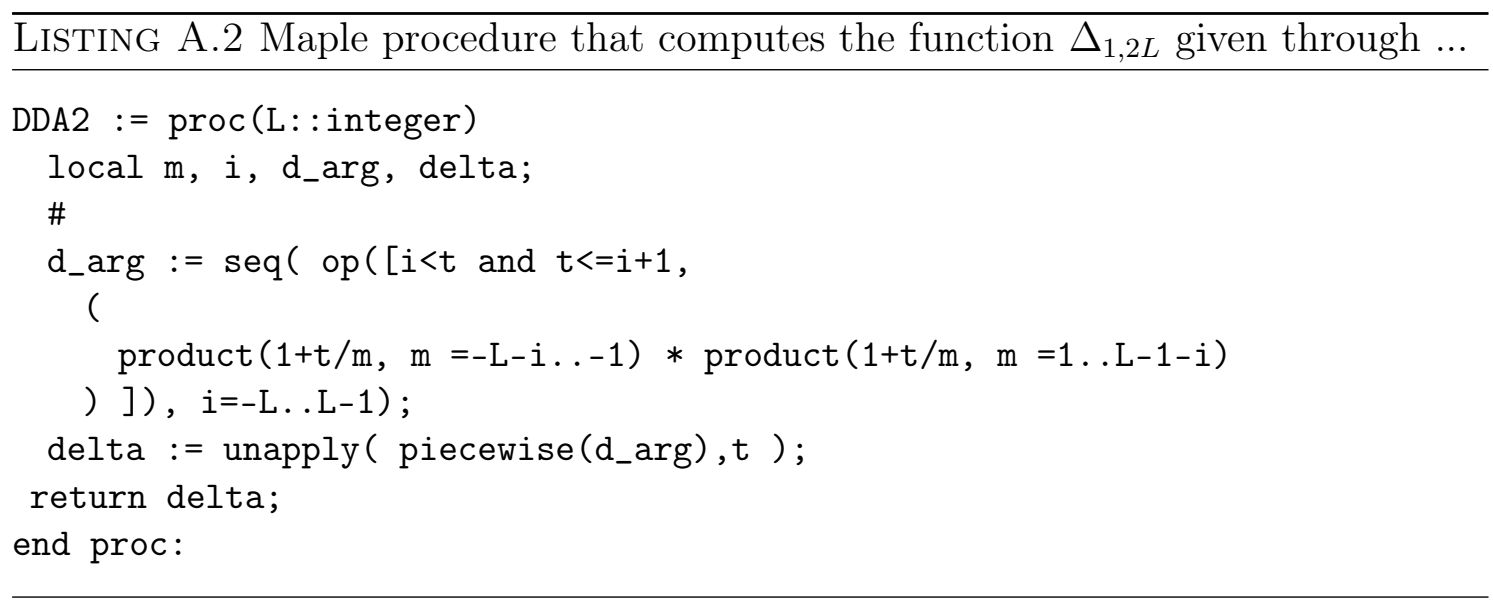




\section{Bibliography}

[1] M. Abramowitz and I. Stegun. Handbook of Mathematical Functions. Dover Publications, New York, 1965.

[2] T. Arens, S. N. Chandler-Wilde, and A. Meier. Integral equation methods for scattering by one-dimensional rough surfaces. 5th International Conference on Mathematical and Numerical Aspects of Wave Propagation, pages 3-13, 2000.

[3] T. Arens, S. N. Chandler-Wilde, and K. O. Haseloh. Solvability and spectral properties of integral equations on the real line. II. $L^{p}$-spaces and applications. J. Integral Equations Appl., 15(1):1-35, 2003. ISSN 0897-3962.

[4] K. E. Atkinson. The numerical solution of integral equations of the second kind, volume 4 of Cambridge monographs on applied and computational mathematics. Cambridge University Press, 1997.

[5] R. P. Beyer and R. J. Leveque. Analysis of a One-Dimensional Model for the Immersed Boundary Method. SIAM Journal on Numerical Analysis, 29 (2):332-364, 1992. URL http://www.jstor.org/view/00361429/di976283/ 97p0024e/0.

[6] B. Birbiad. Fast Integral Methods For Rough Surface Scattering. PhD thesis, Brunel University, 2007.

[7] A. Björck. Numercial Methods for Least Squares Problems. SIAM, 1996.

[8] T. Blu, P. Thévenaz, and M. Unser. MOMS: Maximal-Order Interpolation of minimal support. IEEE Transactions on Image Processing, 10(7):1069-1080, July 2001.

[9] O. P. Bruno and L. A. Kunyansky. A Fast, High-Order Algorithm for the Solution of Surface Scattering Problems: Basic Implementation, Tests, and Applications. Journal of Computational Physics, 169(1):80-110, 2001. ISSN 0021-9991. (doi:10.1006/jcph.2001.6714).

[10] M. D. Buhmann. Radial Basis Functions, volume 12 of Cambridge Monographs on Applied and Computational Mathematics. Cambridge University Press, 2003. 
[11] P. L. Butzer and R. J. Nessel. Fourier Analysis and Approximation Vol.I One.dimensional Theory, volume 40 of Mathematische Reihe. Birkhäuser Verlag, 1971.

[12] L. F. Canino, J. J. Ottusch, M. A. Stalzer, J. L. Visher, and S. M. Wandzura. Numerical Solution of the Helmholtz Equation in 2D and 3D using a high-order Nystroem Discretization. Journal of Computational Physics, 146:627-663, 1998. (doi:10.1006/jcph.1998.6077).

[13] S. N. Chandler-Wilde and M. Lindner. Boundary integral equations on unbounded rough surfaces: Fredholmness and the finite section method. accepted for publication: Journal of Integral Equations and Applications, 2006.

[14] S. N. Chandler-Wilde and P. Monk. Existence, Uniqueness, and Variational methods for scattering by unbounded rough surfaces. SIAM Journal on Mathematical Analysis, 37(2):598-618, 2005.

[15] S. N. Chandler-Wilde and C. R. Ross. Uniqueness results for direct and inverse scattering by infinite surfaces in a lossy medium. Inverse Problems, 10:10631067, 1995.

[16] S. N. Chandler-Wilde and C. R. Ross. Scattering by Rough Surfaces: the Dirichlet Problem for the Helmholtz Equation in a Non-locally Perturbed Halfplane. Mathematical Methods in the Applied Sciences, 19(12):959-976, 1996.

[17] S. N. Chandler-Wilde and B. Zhang. Electromagnetic scattering by an inhomogeneous conducting or dielectric layer on a perfectly conducting plate. Proceedings of the Royal Society A: Mathematical and Engineering Sciences, 454(1970):519-542, 1998. (doi:10.1098/rspa.1998.0173).

[18] S. N. Chandler-Wilde, C. R. Ross, and B. Zhang. Scattering by infinite onedimensional rough surfaces. R. Soc. Lond. Proc. Ser. A Math. Phys. Eng. Sci, 455(1990):3767-3787, 1999. ISSN 1364-5021.

[19] S. N. Chandler-Wilde, B. Birbiad, and A. Meier. A BMIA Canonical Grid Method for Scattering by Rough Surfaces. Technical Report TR/03/02, Brunel University, January 2002.

[20] S. N. Chandler-Wilde, E. Heinemeyer, and R. Potthast. On invertibility and the choice of coupling parameter in a Brakhage-Werner-type integral equation formulation for rough surface scattering. In K. Chen, editor, Advances in Boundary Integral Methods, Proceedings of the Fifth UK Conference on Boundary Integral Methods, pages 164-173. Liverpool University Press, 2005. ISBN 0906370396. 
[21] S. N. Chandler-Wilde, E. Heinemeyer, and R. Potthast. Acoustic scattering by mildly rough unbounded surfaces in three dimensions. SIAM Journal on Applied Mathematics, 66(3):1002-1026, 2006. (doi:10.1137/050635262).

[22] S. N. Chandler-Wilde, E. Heinemeyer, and R. Potthast. A well-posed integral equation formulation for three-dimensional rough surface scattering. Proc. $R$. Soc. A, 462:3683-3705, 2006. (doi:10.1098/rspa.2006.1752).

[23] D. Colton and R. Kress. Inverse Acoustic and Electromagnetic Scattering Theory, volume 93 of Applied Mathematical Sciences. Springer-Verlag, Berlin, 2nd edition, 1992. ISBN 3-540-55518-8.

[24] D. L. Colton and R. Kress. Integral equation methods in scattering theory. Pure and Applied Mathematics. John Wiley \& Sons Inc., New York, 1983. ISBN 0471-86420-X. A Wiley-Interscience Publication.

[25] J. A. DeSanto and P. Martin. On the derivation of boundary integral equations for scattering by an infinite one-dimensional rough surface. Journal of the Acoustical Society of America, 102(1):67-77, 1997.

[26] J. A. DeSanto and P. Martin. On the derivation of boundary integral equations for scattering by an infinite two-dimensional rough surface. Journal of Mathematical Physics, 39(2):894-912, 1998.

[27] A. Erdélyi. Tables of Integral Transforms (Part II). McGraw-Hill, London, 1954.

[28] L. C. Evans. Partial Differential Equations, volume 19 of Graduate studies in mathematics. Providence, Rhode Island (American Mathematical Society), 2002 .

[29] N. Günter. Die Potentialtheorie und ihre Anwendung auf Grundaufgaben der mathematischen Physik. Teubner Leipzig, 1957.

[30] K. Haseloh. Second Kind Integral Equations on the Real Line: Solvability and Numerical Analysis in Weighted Spaces. doctoral thesis, University of Hannover, 2004. URL http://nbn-resolving.de/urn/resolver.pl?urn=urn: nbn: de:gbv:089-4600026272.

[31] E. Heinemeyer, M. Lindner, and R. Potthast. Convergence and numerics of a multi-section method for scattering by three-dimensional rough surfaces. SIAM J. Numerical Analysis, 46(4):1780-1798, 2008. (doi:10.1137/060673321).

[32] K. Jürgens. Lineare Integraloperatoren. B.G. Teubner Stuttgart, 1970. 
[33] R. Kress. Interpolation auf einem unendlichen Intervall. Computing, 6(3-4): 274-288, September 1970. (doi:10.1007/BF02238812).

[34] R. Kress. Numerical Analysis, volume 181 of Graduate Texts in Mathematics. Springer, 1998.

[35] R. Kress. Linear integral equations, volume 82 of Applied Mathematical Sciences. Springer-Verlag, New York, second edition, 1999. ISBN 0-387-98700-2.

[36] R. Kussmaul. Ein numerisches Verfahren zur Loesung des Neumannschen Aussenraumproblems fuer die Helmholtzsche Schwingungsgleichung. Computing, 4:246-273, 1969.

[37] T. M. Lehmann, C. Gönner, and K. Spitzer. Survey: Interpolation Methods in Medical Image Processing. EEEE Transactions on Medical Imaging, 18(11): 1049-1075, November 1999.

[38] M. Lindner. Infinite Matrices and their Finite Sections: An introduction to the Limit Operator Method. Frontiers in Mathematics. Birkhäuser Verlag, Basel, Boston, Berlin, 2006.

[39] W. McLean. Strongly Elliptic Systems and Boundary Integral Equations. Cambridge University Press, 2000. ISBN 052166375X.

[40] A. Meier. Numerical Treatment of integral equations on the real line with application to acoustic scattering by unbounded rough surfaces. pages 1-194, 2001.

[41] A. Meier and S. Chandler-Wilde. On the stability and convergence of the finite section method for integral equation formulations of rough surface scattering. Mathematical Methods in the Applied Sciences, 24:209-232, 2001.

[42] A. Meier, T. Arens, S. Chandler-Wilde, and A. Kirsch. A Nystroem Method for a class of integral equations on the real line with applications to scattering by diffraction gratings and rough surfaces. Journal of Integral Equations and Applications, 12(3):32, 2000.

[43] S. Mikhlin. Mathematical Physics, an Advanced Course. North-Holland Publishing Company, 1970.

[44] J. A. Ogilvy. Wave scattering from rough surfaces. Reports on Progress in Physics, 50:1553-1608, 1987.

[45] K. Pak, L. Tsang, C. H. Chan, and J. Johnson. Backscattering enhancement of electromagnetic waves from two-dimensional perfectly conducting random rough surfaces based on Monte Carlo simulations. J. Opt. Soc. Am. A, 12(11): 2491-2499, 1995. 
[46] C. S. Peskin. The immersed boundary method. Acta Numerica, pages 479-517, 2002. (doi:10.1017/S0962492902000077).

[47] V. S. Rabinovich, S. Roch, and B. Silbermann. Limit Operators and Their Applications in Operator Theory, volume 150 of Operator Theory: Advances and Applications. Birkhaeuser, 2004.

[48] M. Reed and B. Simon. Methods of modern mathematical physics: Part I Functional analysis. Academic Press, 1972.

[49] M. Reed and B. Simon. Methods of modern mathematical physics: Part II Fourier Analysis, Self-Adjointness. Academic Press, New York, 1975.

[50] C. Ross. Direct and Inverse Scattering by Rough Surfaces. PhD thesis, Brunel University, 1996.

[51] T. W. Sag and G. Szekeres. Numerical Evaluation of High-Dimensional Integrals. Mathematics of Computation, 18(86):245-253, 1964.

[52] M. Saillard and A. Sentenac. Rigorous solutions for electromagnetic scattering from rough surfaces. Waves in random media, 11:R103-R173, 2001.

[53] E. M. Stein and G. Weiss. Introduction to Fourier Analysis on Euclidean Spaces. Princeton Mathematical Series. Princeton University Press, 1971.

[54] P. Thévenaz, T. Blu, and M. Unser. Interpolation Revisited. IEEE Transactions On Medical Imaging, 19(7):739-758, 2000.

[55] A. Voronovich. Wave scattering from Rough Surfaces. Springer Berlin, 1998.

[56] J. Waldén. On the Approximation of Singular Source Terms in Differential Equations. Numerical Methods for Partial Differential Equations, 15(4):503-520, 1999. (doi:10.1002/(SICI)1098-2426(199907)15:4<503::AIDNUM6>3.0.CO;2-Q).

[57] K. F. Warnick and W. C. Chew. Numerical simulation methods for rough surface scattering. Waves in random media, 11:R1-R30, 2001.

[58] J. Weidmann. Lineare Operatoren in Hilberträumen: Teil I Grundlagen. Mathematische Leitfäden. B.G. Teubner, first edition, 2000.

[59] H. Wendland. Scattered Data Approximation, volume 17 of Cambridge Monographs on Applied and Computational Mathematics. Cambridge University Press, 2005.

[60] D. Werner. Funktionalanalysis. Springer-Verlag, third edition, 2000. ISBN 3-540-67645-7. 
[61] W. Wladimiriow. Gleichungen der mathematischen Physik. VEB Deutscher Verlag der Wissenschaften, 1972.

[62] M. Y. Xia, C. H. Chan, S. Q. Li, B. Zhang, and L. Tsang. An efficient algorithm for electromagnetic scattering from rough surfaces using a single integral equation and multilevel sparse-matrix canonical-grid method. IEEE Trans. Antennas and Propagation, 51(6):1142-1149, 2003. ISSN 0018-926X. (doi:10.1109/TAP.2003.812238).

[63] B. Zhang and S. N. Chandler-Wilde. Integral equation methods for scattering by infinite rough surfaces. Mathematical Methods in the Applied Sciences, 24: 463-488, 2003. 


\section{List of Symbols}

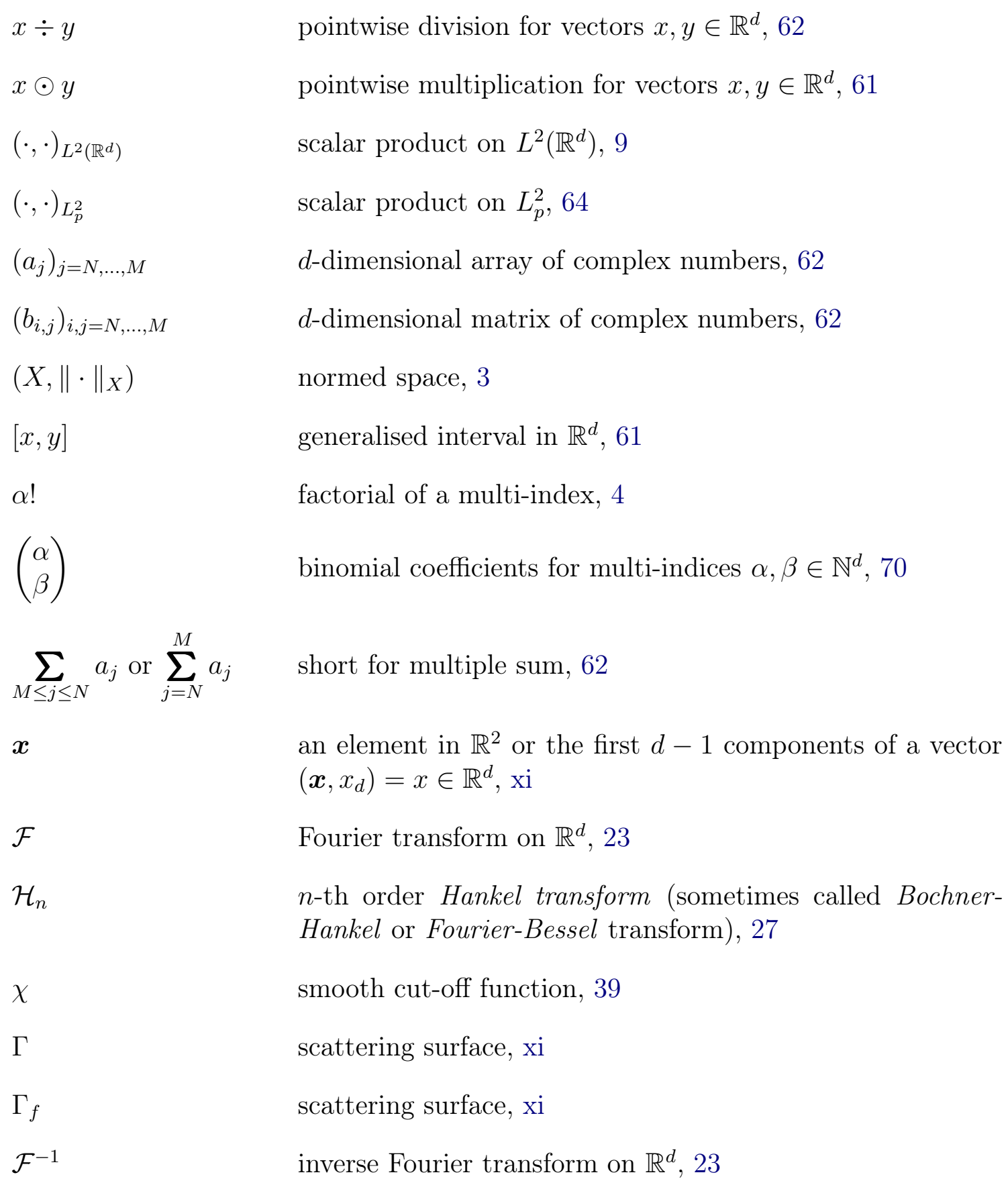


$\kappa$

$\kappa_{i}$

$\kappa_{r}$

$\langle x, y\rangle$

$|\alpha|$

$|\cdot|{ }_{B C^{0, \alpha}}$

$\mathcal{S}\left(\mathbb{R}^{d}\right)$

$\nu$

$\partial^{\alpha}$

$\Phi(\cdot, \cdot)$

$\mathbb{R}_{>0}^{d}$

$\sharp(x)$

$\tilde{\mathbb{C}}$

$\|A\|_{X \rightarrow Y}$

$\|(\cdot, \cdot)\|_{X \times Y}$

$\|\cdot\|_{X}$

$\|\cdot\|_{B C\left(\mathbb{R}^{d}\right)}$

$\|\cdot\|_{B C^{0, \alpha}}$

$\|\cdot\|_{B C^{1, \alpha}}$

$\|\cdot\|_{B C_{p}^{n}\left(\mathbb{R}^{d}\right)}$

$\|\cdot\|_{L^{p}\left(\mathbb{R}^{d}\right)}$

$\|\cdot\|_{L^{\infty}\left(\mathbb{R}^{d}\right)}$

$B\left(r_{1}, r_{2} ; \boldsymbol{x}\right)$

$B C\left(\mathbb{R}^{d}\right)$ wavenumber, xii

imaginary part of wavenumber, xii

real part of wavenumber, xii

standard scalar product of two vectors $x, y \in \mathbb{R}^{d}, 61$

order of a multi-index, 4

the Hölder semi-norm, 6

the Schwartz space of rapidly decreasing functions, 5

the unit normal vector directed into $D$, xv

partial derivatives, 5

the fundamental solution to the Helmholtz equation in $\mathbb{R}^{3}$, xiii

all elements of $\mathbb{R}^{d}$ width non-negative components, 61

the product of the components of an vector $x \in \mathbb{R}_{>0}^{d}, 61$

admissible set of wavenumbers, xii

operator norm of an operator $A: X \rightarrow Y, 3$

norm on the product space $X \times Y, 3$

norm on $X, 3$

supremum norm, 4

the Hölder norm, 5

the norm on $B C^{1, \alpha}\left(\mathbb{R}^{d}\right), 6$

norm on $B C_{p}^{n}\left(\mathbb{R}^{d}\right), 69$

the $L^{p}$-norm, 6

the $L^{\infty}$-norm, 7

annulus with radii $r_{1}<r_{2}$ and centre $\boldsymbol{x}$, xiv

the space of bounded and continuous real- or complex-valued functions on $\mathbb{R}^{d}, 4$ 


$$
\begin{aligned}
& B C^{k}(\Gamma) \\
& B C^{0, \alpha}\left(\mathbb{R}^{d}\right) \\
& B C^{1, \alpha}\left(\mathbb{R}^{d}\right) \\
& B C^{\infty}\left(\mathbb{R}^{d}\right) \\
& B C^{k}\left(\mathbb{R}^{d}\right) \\
& B C_{p}^{n}\left(\mathbb{R}^{d}\right) \\
& B C_{p}^{n}\left(\mathbb{R}^{d} \times \mathbb{R}^{d}\right) \\
& B C_{\infty}\left(\mathbb{R}^{d}\right) \\
& B L(X) \\
& B L(X ; Y) \\
& C_{0}^{n}\left([0,1]^{d}\right) \\
& C^{\infty}\left(\mathbb{R}^{d}\right) \\
& C_{\text {comp }}^{\infty}\left(\mathbb{R}^{d}\right), \mathcal{D}\left(\mathbb{R}^{d}\right) \\
& C^{k}\left(\mathbb{R}^{d}\right) \\
& C_{\text {comp }}^{k}\left(\mathbb{R}^{d}\right) \\
& \text { D } \\
& D^{0}
\end{aligned}
$$




\begin{tabular}{|c|c|}
\hline$D_{f}$ & medium of wave propagation, xi \\
\hline$e_{j}$ & $j$ 'th basis vector in $\mathbb{R}^{d}, 61$ \\
\hline$f * g$ & the convolution product, 34 \\
\hline$f^{-}, f^{+}$ & lower and upper bound of the surface height function $f$, xi \\
\hline$G(\cdot, \cdot)$ & $\begin{array}{l}\text { the half-space Dirichlet Green's function for the Helmholtz } \\
\text { equation, xv }\end{array}$ \\
\hline$G L(X)$ & set of all invertible operators in $B L(X), 3$ \\
\hline$I_{f}$ & $\begin{array}{l}\text { isomorphism that maps functions spaces on the boundary } \\
\Gamma_{f} \text { to function spaces on } \mathbb{R}^{2}, 10\end{array}$ \\
\hline$J$ & the surface area-element, 10 \\
\hline$J_{n}$ & Bessel functions of the first kind of order $n, 27$ \\
\hline$K$ & the double-layer potential operator, xv \\
\hline$L_{p}^{2}$ & short for $L_{p}^{2}\left(\mathbb{R}^{d}\right), 64$ \\
\hline$L_{p}^{2}\left(\mathbb{R}^{d}\right)$ & $\begin{array}{l}\text { the space of all complex valued, Lebesgue square integrable } \\
\text { multi-periodic function with period vector } p \in \mathbb{R}_{>0}^{d}, 64\end{array}$ \\
\hline$L^{p}\left(\mathbb{R}^{d}\right)$ & $\begin{array}{l}\text { the Lebesgue space of real- or complex-valued functions that } \\
\text { are integrable to the power } p \text { on } \mathbb{R}^{d}, 6\end{array}$ \\
\hline$L^{p}\left(\mathbb{R}_{\geq 0}, r d r\right)$ & the weighted $L^{p}$-space on the positive half-line, 10 \\
\hline$L^{\infty}\left(\mathbb{R}^{d}\right)$ & the space of essentially bounded functions on $\mathbb{R}^{d}, 6$ \\
\hline$L_{f}$ & $\begin{array}{l}\text { the Lipschitz constant of } f \text { (the maximum surface slope of } \\
\left.\Gamma_{f}\right), 11\end{array}$ \\
\hline$p, p^{\prime}$ & conjugate pairs of exponents, 7 \\
\hline$P_{f}$ & the natural parametrisation of the scattering surface $\Gamma, 10$ \\
\hline$Q^{d}[f]$ & the $d$-dimensional integral $\int_{\mathbb{R}^{d}} f(x) d x, 69$ \\
\hline$Q_{h}^{d}[f]$ & the $d$-dimensional quadrature operator $h^{d} \sum_{j \in \mathbb{Z}^{d}} f(h j), 69$ \\
\hline$S$ & the single-layer potential operator, $\mathrm{xv}$ \\
\hline$u$ & total field, xii \\
\hline
\end{tabular}




$\begin{array}{ll}u^{i} & \text { incident field, xii } \\ u^{s} & \text { scattered field, xii } \\ X \cap Y & \text { intersection of two normed spaces } X, Y, 3 \\ X(\Gamma) & \text { short for } L^{2}(\Gamma) \cap B C(\Gamma), \text { xxii } \\ x<y & \text { partial ordering for } x, y \in \mathbb{R}^{d}, 61 \\ x \leq y & \text { partial ordering for } x, y \in \mathbb{R}^{d}, 61 \\ X \times Y & \text { product space of two normed space } X, Y, 3 \\ x^{\alpha} & \text { pointwise exponentiation, } 4 \\ X_{\infty}(\Gamma) & \text { short for } L^{2}(\Gamma) \cap B C_{\infty}(\Gamma), \text { xxii } \\ y^{\prime} & \text { the mirrored point, xv } \\ z & \text { source point of incident point source, xiii }\end{array}$




\title{
Lebenslauf
}

\author{
Name: $\quad$ Eric Heinemeyer \\ Geburtsdatum: $\quad 15.02 .1976$ \\ Geburtsort: Peine \\ Nationalität: deutsch \\ Schulbildung: \\ 1982 - 1996 Grundschule Groß Bülten \\ OS Ilsede/OS Lehrte Süd \\ Gymnasium Lehrte \\ Okt. 93 - Juni $94 \quad$ Austausschüler an der McLoughlin Union Highschool in \\ Milton-Freewater, Oregon (USA)
}

Wehr-/Ersatzdienst:

1996 - 1997

Zivildienst im evangelischen Altersheim Lehrte

Wissenschaftliche Ausbildung:

Okt. 1997 - Jan. 2004 Studium der Mathematik mit Nebenfach theoretische Physik

15. Okt 1999

WS $00 / 01$

WS02/03

SS 03

29. Jan. 2004 an der Georg-August Universität Göttingen

Vordiplom mit der Note „Gut “

Studentische Hilfskraft

Analysis I

Numerik gewöhnlicher Differentialgleichungen

Numerische Mathematik II

Mathematik-Diplom (Note, sehr gut")

Diplomarbeitsthema: „Direkte und Inverse Randwertprobleme aus der Modellierung elektrostatischer Materialprüfungsverfahren "

Betreuer: Prof. Dr. R. Kreß

Apr. 2004 - Sep. 2004 Wissenschaftliche Hilfskraft am Institut für Numerische und Angewandte Mathematik, Universität Göttingen

Okt. 2004 - Sep. 2007 Beginn der Promotion unter der Anleitung von Herrn Prof. Potthast als Stipendiat im Graduiertenkolleg 1023 „Identifikation in mathematischen Modellen: Synergie stochastischer und numerischer Methoden".

Jul. 2005 - Sep. 2005 Forschungsprojekt zusammen mit dem deutschen Zentrum für Luft- und Raumfahrt (DLR), Standort Göttingen, über Rekonstruktionen spiegelnder Oberflächen. 